\title{
Cholinergic modulation of
}

stimulus-specific adaptation and prediction error responses in the auditory cortex of the rat

\section{Cristian Andrés Aedo-Sánchez}
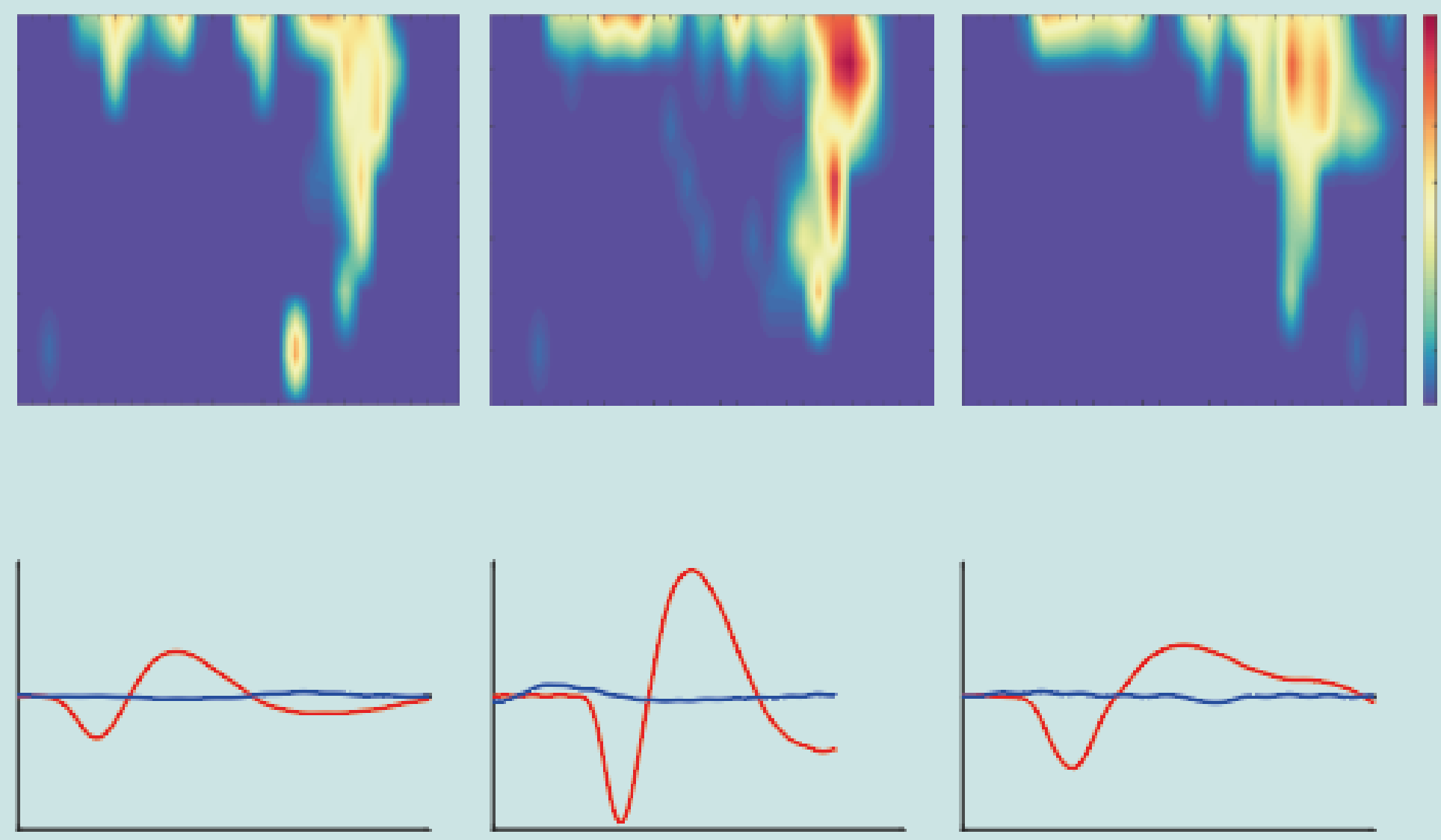

PhD Thesis

2020 
UNIVERSITY OF SALAMANCA

Institute of Neuroscience of Castilla y León (INCyL)

Cognitive and Auditory Neuroscience Laboratory (CANE-LAB)

Doctoral Program in Neurosciences

INSTITUTO DE

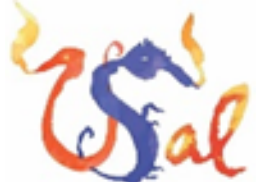

800 Años

VNIVERSIDAD

BSALAMANCA



\section{CHOLINERGIC MODULATION OF STIMULUS-SPECIFIC ADAPTATION AND PREDICTION ERROR RESPONSES IN THE AUDITORY CORTEX OF THE RAT}

Thesis submitted

By

Cristian Andrés Aedo-Sánchez

To obtain the degree of

$\mathrm{PhD}$ in Neuroscience

and International Doctor Mention

Under the supervision of:

Dr. Manuel Sánchez Malmierca

and

Dr. David Pérez-González

Salamanca, November 2020 
Manuel Sánchez Malmierca, catedrático del Departamento de Biología Celular y Patología de la Universidad de Salamanca y director del Instituto de Neurociencias de Castilla y León.

David Pérez-González, investigador postdoctoral senior del Instituto de Neurociencias de Castilla y León.

\section{CERTIFICAN}

Que la tesis doctoral titulada:

CHOLINERGIC MODULATION OF STIMULUS-SPECIFIC ADAPTATION AND PREDICTION ERROR RESPONSES IN THE AUDITORY CORTEX OF THE RAT

Ha sido redactada en inglés, contiene un resumen en español y describe el trabajo de investigación realizado por Cristian Andrés Aedo Sánchez bajo nuestra dirección durante los últimos 3 años.

La memoria de este estudio recoge un análisis detallado y exhaustivo de la modulación colinérgica en las respuestas de adaptación específica a estímulos y de codificación predictiva de la corteza auditiva en un modelo animal. Los datos presentados en esta memoria constituyen una aportación original y podemos afirmar que ponen de manifiesto un gran avance y progreso en el área de la Neurociencia.

Por todo ello, consideramos que esta tesis reúne los requisitos, calidad y rigor científicos necesarios para que sea defendida en la Universidad de Salamanca como trámite para que Don Cristian Andrés Aedo Sánchez opte al grado de 'Doctor en Neurociencias con mención doctor internacional' por la Universidad de Salamanca.

Y para que así conste, firmamos el presente certificado en Salamanca, a 21 de octubre de 2020.

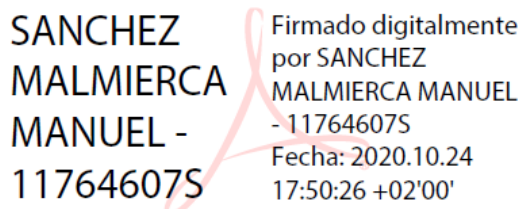

Manuel Sánchez Malmierca

\begin{tabular}{|c|c|}
\hline PEREZ & Firmado \\
\hline ZALE & $\begin{array}{l}\text { digitalmente por } \\
\text { PEREZ GONZALEZ }\end{array}$ \\
\hline וג & DAVID - 70863212M \\
\hline 217 & $\begin{array}{l}\text { Fecha: } 2020.10 .25 \\
17: 51: 24+01^{\prime} 00^{\prime}\end{array}$ \\
\hline
\end{tabular}

David Pérez-González 
This work has been funded by the Government of Spain, MINECO (SAF2016-75803P).

Cristian Andrés Aedo-Sánchez has received the sponsorship of the University of Chile.

Este trabajo ha sido financiado por el Gobierno de España, MINECO (SAF201675803-P).

Cristian Andrés Aedo-Sánchez ha recibido el patrocinio de la Universidad de Chile. 
Doctoral thesis supervised by

\section{Dr. Manuel Sánchez Malmierca}

And

\section{Dr. David Pérez-González}

Cognitive and Auditory Neuroscience Laboratory (CANE-Lab),

Institute of Neuroscience of Castilla y León.

University of Salamanca 
A mis padres Juan Luis Aedo y Liliana Sánchez, por todo su infinito amor, cariño y enseñanzas. 


\section{Agradecimientos}

En primer lugar, agradezco enormemente a Manolo por darme la oportunidad de realizar mi tesis. Estoy muy agradecido de su orientación, rigurosidad, optimismo en los momentos difíciles y sobretodo su calidad de ser humano. También agradezco muchísimo a David, quien me ha enseñado y guiado en todo este proceso con sus conocimientos, apoyo y paciencia.

Gracias a todos mis compañeros de laboratorio: Lorena, Gloria, Flora, Ana, Camilo, Catalina, Guillermo, Blanca, Antonio y María por todas sus risas, almuerzos enseñanzas, apoyo y discusiones que muchas veces continuaban en algún bar de la ciudad. Han sido un apoyo enorme en mi estadía en la bella Salamanca y se han convertido en mi segunda familia en todos estos años. También agradezco a integrantes de otros laboratorios y personal del INCYL por alegrar mis mañanas con una conversación matutina o un simple saludo de buenos días.

Gracias al apoyo de la Universidad de Chile y el Departamento de Tecnología Médica por apoyar el desarrollo de mi doctorado. A los profesores Elizabeth Pavez, Luis Robles y Paul H. Delano les agradezco enormemente su apoyo, motivación y enseñanzas para que pudiese desarrollarme en el mundo de la investigación y las neurociencias. A la profesora Inés Pepper por apoyar mis estudios en la Universidad de Salamanca y a todos mis compañeros de la mención de otorrinolaringología, especialmente a Gonzalo Cuellar y Enzo Aguilar por brindarme su amistad y soporte durante todos estos años.

Finalmente, agradezco a todas las personas y amistades que me han alentado desde Chile. Muchas gracias a mis padres Juan Luis Aedo y Liliana Sánchez por todo su apoyo, amor y comprensión. Todas sus enseñanzas y cariño infinito han permitido desarrollarme como el profesional y persona que soy ahora. También agradezco a Paulina Krippel por todo su amor durante estos años. El habernos conocido y recorrer hermosos paisajes ha sido uno de los mejores regalos que he podido tener.

Para el desarrollo de esta tesis, he contado con la estrecha colaboración de David Pérez-González en el capítulo I y de Camilo Díaz-Morado en el capítulo III. Agradezco su apoyo y generosidad en la realización de estos trabajos y que puedan formar parte de mi tesis doctoral. 


\section{ORIGINAL PUBLICATIONS}

This thesis is based on the following publications:

Peer-reviewed published article:

Pérez-González, D., Parras, G.G., Morado-Díaz, C.J., Aedo-Sánchez, C., Carbajal, G. V., Malmierca, M.S., 2020. Deviance detection in physiologically identified cell types in the rat auditory cortex. Hear. Res.

https://doi.org/10.1016/j.heares.2020.107997

Article under review:

Aedo-Sánchez, C., Pérez-González, D C., Malmierca, M.S., 2020.The Role of Cholinergic Neuromodulation in Forwarding Prediction Error in the Auditory Cortex. Under revision after first review in eLife. (ms \# 26-05-2020-RA-eLife-59347) 


\section{Abbreviations.}

A1, primary auditory cortex.

$A C$, auditory cortex.

ACh, acetylcholine.

AAF, anterior auditory field.

$A C$, auditory cortex.

CAS, cascade sequence.

$\mathrm{CF}$, characteristic frequency.

CSI, common stimulus-specific adaptation index.

DEV, deviant.

EEG, electroencephalography.

FRA, frequency response area.

IC, inferior colliculus.

iMM, index of neural mismatch.

iPE, index of prediction error.

iRS, index of repetition suppression.

MGB, medial geniculate body.

MMN, mismatch negativity.

nAChR, nicotinic acetylcholine receptor.

mAChR, muscarinic acetylcholine receptor.

PAF, posterior auditory field.

PSTH, peri-stimulus time histogram.

$\mathrm{PV}$, parvalbumin interneurons.

SSA, stimulus-specific adaptation.

SPL, sound pressure level.

SRAF, supra-rhinal auditory field.

SST, somatostatin interneurons.

STD, standard.

VAF, ventral auditory field.

VIP, vasoactive intestinal peptide. 
1. Abbreviations

2. Abstract

3. Introduction

- The auditory pathway

- Auditory cortex

- Lemniscal and non-lemniscal pathways in the auditory system

- Stimulus-specific adaptation in the auditory system

- $\quad$ Mismatch negativity

- Stimulus-specific adaptation and mismatch negativity

- Predictive coding framework

- Acetylcholine

- Acetylcholine in sensorial systems

5. Objectives

6. Results

- Section I: deviance detection in physiologically identified cell types in the rat auditory cortex

- $\quad$ Regular and fast spiking of auditory cortical neurons

- Spike amplitude, cortical field and cortical layer localization

- $\quad$ The prediction error and repetition supression indices

- Section II: Acetylcholine modulates SSA and prediction error response in auditory rat cortex

- Electrophysiological responses in AC using oddball paradigms

- The effect of ACh on SSA in AC and cholinergic antagonist

- ACh effect on SSA as a function of topographic distribution in auditory cortical layers and fields

- ACh affects neuronal mismatch, increasing prediction error but not repetition suppression in cascade and many-standard paradigms. 
- Section III: Effect of behaviorally relevant sounds in deviant detection and prediction error responses

- Behavioral responses to the oddball paradigm. 83

- Frequency contrast impact on behavioral responses to oddball 86 paradigm

- Responses to many-deviants sequence 89

- Behavioral effects on neural responses in auditory cortex. 91

7. General Discussion 95

$\begin{array}{ll}\text { 8. Conclusions } & 113\end{array}$

$\begin{array}{lr}\text { 9. References } & 114\end{array}$

$\begin{array}{lr}\text { 10. Publications } & 139\end{array}$

$\begin{array}{ll}\text { 11. Spanish Summary } & 206\end{array}$ 


\section{ABSTRACT}

A fundamental property of sensory systems is their ability to detect novel stimuli in the environment. The auditory brain contains neurons that decrease their response to repetitive sounds but increase their firing rate against novel or deviant stimuli; the difference between both responses is known as stimulusspecific adaptation (SSA) or neuronal mismatch. This thesis describes 1) the properties of excitatory (fast spiking) and inhibitory (regular spiking) auditory cortical neurons, 2) how acetylcholine (ACh) modulates SSA in the rat auditory cortex and 3) how rats can discriminate behaviorally relevant novel sounds. Results show that 1) fast spiking and regular spiking neurons show similar amounts of deviance detection, 2) ACh increases SSA by $~ 31 \%$ in the auditory cortex. Importantly, ACh increased the neuronal firing rate in response to deviant tones only, and only the prediction error component was affected. This thesis also demonstrates that $\mathrm{ACh}$ increases the precision of prediction error signaling and is mediated by muscarinic receptors, gating prediction errors to hierarchically higher processing levels. Finally, 3) the training on the auditory discrimination task had effects on neuronal activity, increasing the deviant detection, neuronal mismatch and prediction error responses. 


\section{INTRODUCTION}

The information from our environment comes mainly from our sensory systems.

One of the main characteristics of these systems is the detection of novel stimuli, a common element in our nervous system. The ability to respond to new stimuli that occur in the environment is extremely important for survival (Malmierca, 2014). The presence of a novel stimulus triggers a cascade of neural events including perception, attention, learning and memory. The auditory system has the ability to detect sounds that violate the regularity established by a sound stream in the auditory scene (for example, the sound of an emergency alarm in a restaurant) (Bendixen, 2014; Szabó et al., 2016). This ability is very important for the perception of our environment and would be altered in some neurodegenerative diseases and neuropsychiatric disorders such as Alzheimer's disease, schizophrenia, or autism (Hardy et al., 2020; Matthews et al., 2013; Ruzzoli et al., 2016).

In this thesis, I describe the general responses to stimulus-specific adaptation in the auditory cortex of the rat, how the cholinergic system modulates the novelty response and what ACh receptors mediate this action in the primary and secondary rat auditory cortex, utilizing the microiontophoresis technique. I also describe how the levels of prediction of error are modulated by acetylcholine. Finally, I study how rats can discriminate behaviorally relevant novel sounds. 


\section{The auditory pathway}

Anatomically and functionally, we can divide the peripheral auditory system into three parts: external, middle, and inner ear (for review, see (Malmierca, 2015); (Pickles, 2015)). The external ear consists of the pinna and the external ear canal and its main function is to capture and steer acoustic stimuli to the tympanic membrane. The middle ear consists of three bones: the hammer (malleus), the anvil (incus) and the stirrup (stapes), the oval window, the round window and the Eustachian tube. The middle ear transmits sound vibration from the tympanic membrane to the oval window, located in the inner ear. The main function of the middle ear is to be an impedance adjuster, restoring the loss of energy when the sound passes from an aerial medium (air) to a liquid medium (endolymph and perilymph) (Mansour et al., 2013). The inner ear is the innermost part of the ear, which consist of the cochlea, the vestibular system, and the vestibulo-cochlear nerve. The cochlea is a receptor organ that transduces these mechanical signals into electrical ones and that sends this information to the central nervous system, preserving the frequency coding from this point to the auditory cortex (tonotopic organization). At this point, a certain type of sensory cells (inner hair cells) synapse with afferents fibers of the auditory nerve (ribbon synapse) releasing glutamate as a neurotransmitter (Safieddine et al., 2012). The action potentials generated in the inner ear are conducted through the auditory nerve to the cochlear nucleus and from there the information is transmitted in parallel pathways through the ventral, intermediate and dorsal acoustic stria to the nuclei of the brainstem. These nuclei include the superior olive complex and the lateral lemniscus. Then, auditory information converges in the inferior colliculus (located at the midbrain) which is an obligatory relay station before inputs further proceed 
to the medial geniculate body of the thalamus. Next, neurons in the auditory thalamus project to the temporal lobe, where the auditory cortex is located, and the place responsible for conscious auditory perception (Pickles, 2015, Fig. 1).

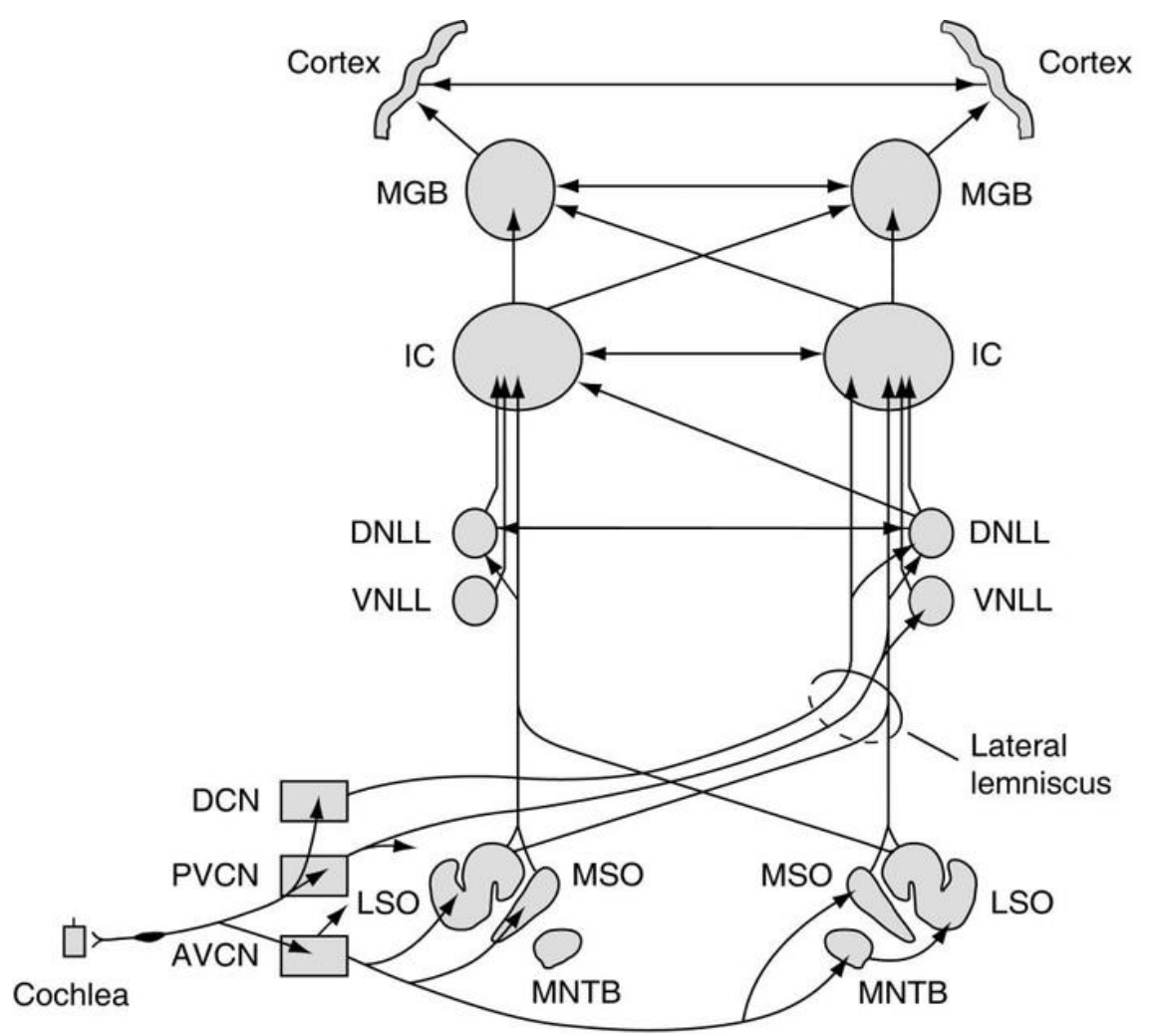

Fig 1. The main ascending pathways of the brainstem. $A C N$, anterior cochlear nucleus; DCN, dorsal cochlear nucleus; PCN, posterior cochlear nucleus; MSO, medial superior olive; LSO, lateral superior olive; MNTB, medial nucleus of the trapezoid body; DNLL, dorsal nucleus of the lateral lemniscus; VNLL, ventral nucleus of the lateral lemniscus; IC, inferior colliculus; MGB, medial geniculated body. Reproduced from Pickles (2015).

\section{Auditory cortex}

From an anatomical point of view, we can define the auditory cortex (AC) as any portion of the cerebral cortex that receives input from the MGB. According to this definition, the AC of most mammals would be located in areas adjacent to the temporal lobe (Malmierca and Hackett; 2010; Hackett, 2015). We can classify the 
AC as primary or secondary according to the anatomical projections it receives or by its electrophysiological response. The primary auditory cortex (core or lemniscal region) presents a tuned response, frequency specificity and short latencies. In contrast, the secondary auditory cortex (belt or non-lemniscal region) shows a less specific frequency response, longer response latencies and nottuned response (Hackett, 2015). Finally, there are associative cortices (parabelt regions) that integrate auditory information with that of other sensory systems (Ghazanfar and Schroeder, 2006).

As opposed to the subcortical auditory centers where their structure and physiology are relatively homologous and well conserved, the structure and physiological properties of the auditory cortex fields varies substantially across species (Hackett, 2015). In the rat AC, five fields have been described: the primary auditory cortex (A1), the posterior auditory field (PAF), the anterior auditory field (AAF), the ventral auditory field (VAF), and the suprarhinal auditory field (SRAF); based on the spatial orientation of tonotopic maps, spectral-tuning characteristics, intensity-tuning characteristics, response thresholds, response latencies, and a comparison of thalamic input sources (Nieto-Diego and Malmierca, 2016; Polley et al., 2007; Profant et al., 2013) (Fig. 2). The major reference to define the extension of these fields is the progression of their characteristic tonotopic gradients. The boundaries between them are defined by inversions or bifurcations of these gradients. This important feature makes it feasible to locate the relative position of each field in a single animal through electrophysiological mapping in vivo (Nieto-Diego and Malmierca, 2016). A1, $\mathrm{AAF}$ and VAF correspond to the primary auditory cortex (lemniscal) while SRAF 
and PAF correspond to the secondary auditory cortex (non lemniscal). A1, AAF and VAF receive projections from the MGV (VAF also receives projections from medial geniculated body of Thalamus). SRAF and PAF receives several projections from the medial geniculated body (Malmierca, 2015; Polley et al., 2007).
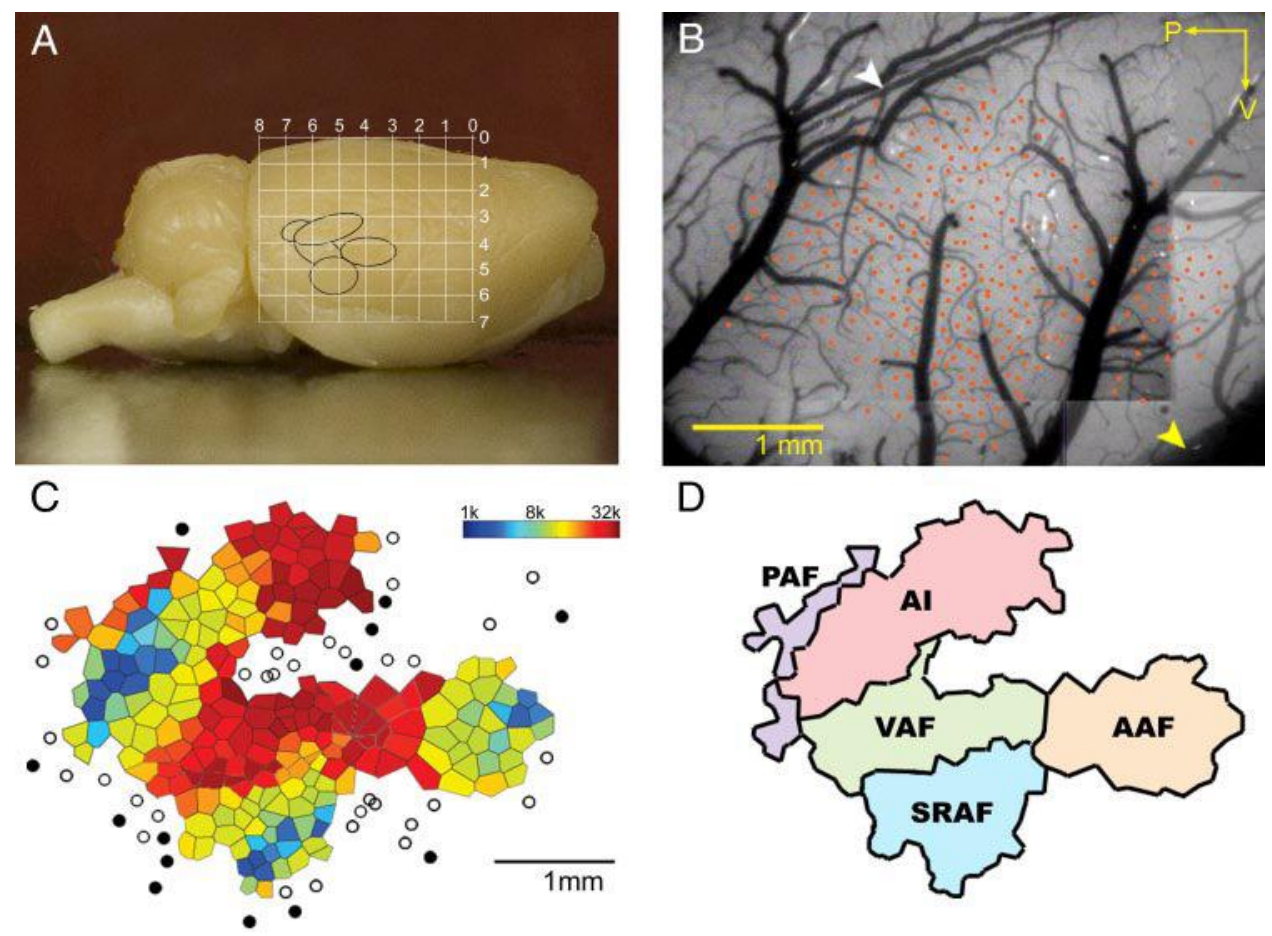

Fig 2. Rat auditory cortex. (A). The rat AC completely exposed in the lateral area of the temporal lobe, between 3 and $7 \mathrm{~mm}$ posterior to bregma. (B) The pattern of vasculature can be used to reference relative electrode positions. (C) Physiological mapping of the whole AC in one rat, showing the tonotopic gradients of characteristic frequency that define the boundaries between different $A C$ areas. (D) Scheme of five different auditory cortical fields identified in the rat: A1, VAF and AAF are primary fields (lemniscal zones), whereas SRAF and PAF are considered higher-order (non-lemniscal zones). Reproduced from Polley et al. (2007).

The cytoarchitecture of the auditory cortex follows the general plan of the cerebral cortex and contains 6 layers: layer I to layer VI (Fig. 3) (For review, see (Malmierca, 2015)). Layer I has very few non-pyramidal type neurons and generates $13 \%$ of the total cortical thickness. Layer II is densely populated by 
smaller, pyramidal and non-pyramidal type neurons. In this layer there are around $24 \%$ of GABAergic interneurons. Layer III is made up of both pyramidal and nonpyramidal cells and has around 24\% GABAergic interneurons. Layer IV (unlike other cortices such as visual or somatosensory) is composed almost exclusively of pyramidal cells, which are more densely packed than layer III and receives afferent projections from the ventral division of the MGB of the thalamus. Layer $V$ is the one with the greatest cortical thickness (approx. 26\%) and is composed mainly of pyramidal cells, receiving afferent projections mainly from the dorsal portion of the MGB of the thalamus and sending efferent projections mainly to the inferior colliculus, medial olive complex, lateral olive complex and cochlear nucleus. (Coomes and Schofield, 2004; King et al., 2011; Schofield and Coomes, 2005). Layer VI is mainly made up of pyramidal cells of different sizes. It receives input from the ventral portion of the MGB of the thalamus. On the other hand, it sends efferent projections to the MGB, which in turn go to the auditory cortex, forming a thalamus-cortex-thalamus feedback loop (Winer and Prieto, 2001). The efferent projections of layers $\mathrm{V}$ and $\mathrm{VI}$ have their origin mainly in the primary auditory cortex (Saldaña, 2015; Schofield, 2011). 


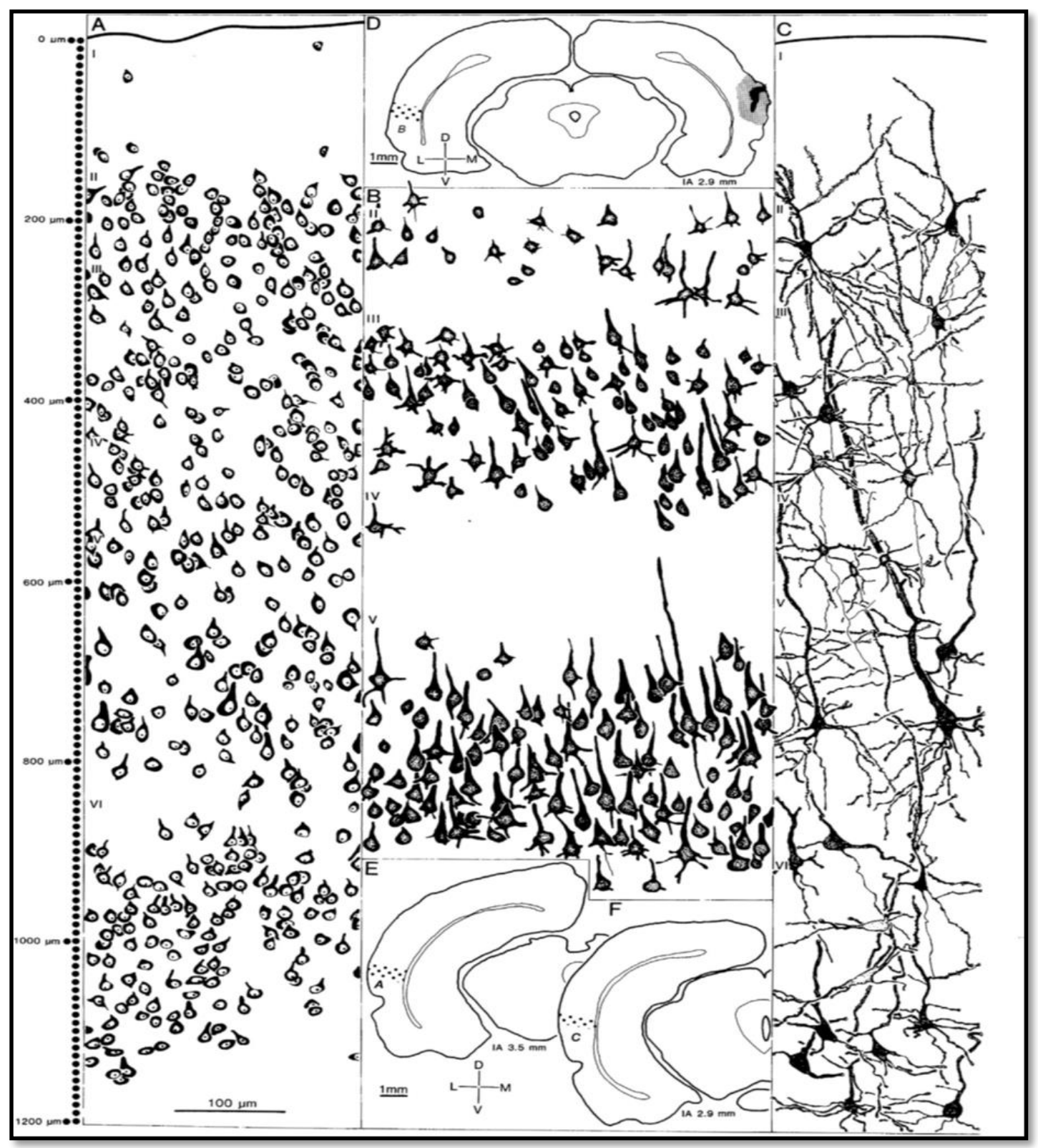

Fig 3. Neuronal types scheme of auditory cortex. Neuronal types and laminar boundaries in the primary auditory cortex (Te1; Au1) seen in Nissl stained sections (A), HRP labeled cells after injections into the contralateral AC and ipsilateral IC (B) and Golgi impregnated material (C). Note different cell types, neuronal density and dendritic branching patterns in each of the six layers. (D-F) insets showing from which panels B, A and C were drawn, respectively. Reproduced from (Games and Winer, 1988).

\section{Lemniscal and non-lemniscal pathways in the auditory system}

As mentioned above, auditory information is transmitted along various hierarchically organized nuclei through the auditory neuroaxis. However, from the 
midbrain, two main pathways of structural and functional characteristics that differ from each other have been distinguished. These pathways have been called "lemniscal" and "non-lemniscal" (Lee and Winer, 2008) which can also be referred to as primaries or non-primary regions, respectively. The response of lemniscal pathway is fundamentally driven by the physical features of the sound. Non-lemniscal divisions are part of a higher order stage of processing, constituting a secondary system capable of processing more complex aspects of the auditory scene analysis and tracking the history of stimulation (Table 1).

\begin{tabular}{|l|l|}
\hline Lemniscal pathway & Non lemniscal pathway \\
\hline Low order stage of processing & High order stage of processing \\
\hline Short response latencies & Long response latencies \\
\hline Sharp frequency tuning & Broad frequency tuning \\
\hline $\begin{array}{l}\text { Greater firing rates and higher } \\
\text { spontaneous activity }\end{array}$ & $\begin{array}{l}\text { Lower firing rates and higher } \\
\text { spontaneous activity }\end{array}$ \\
\hline $\begin{array}{l}\text { Neuronal responses are } \\
\text { fundamentally driven by the physical } \\
\text { features of the sound }\end{array}$ & $\begin{array}{l}\text { Neuronal responses process more } \\
\text { complex aspects of the auditory scene }\end{array}$ \\
\hline
\end{tabular}

Table 1. Summary of the main characteristics of the lemniscal and non-lemniscal pathways

Generally speaking, the lemniscal pathway originates in the central nucleus of the IC (CNIC), where it receives inputs from nuclei of the lateral lemniscus, ascends to the ventral division of the MGB, and projects $A 1, A A F$, and $V A F$ fields of the AC. The non-lemniscal pathway receives its inputs from multiple sources, including non-auditory centers. It arises in the dorsal, lateral and rostral cortices of the IC, projects to the dorsal and medial divisions of the MGB, the output of which is sent to the PAF and SRAF auditory fields of the AC (Fig. 4) (Carbajal and Malmierca, 2018). 


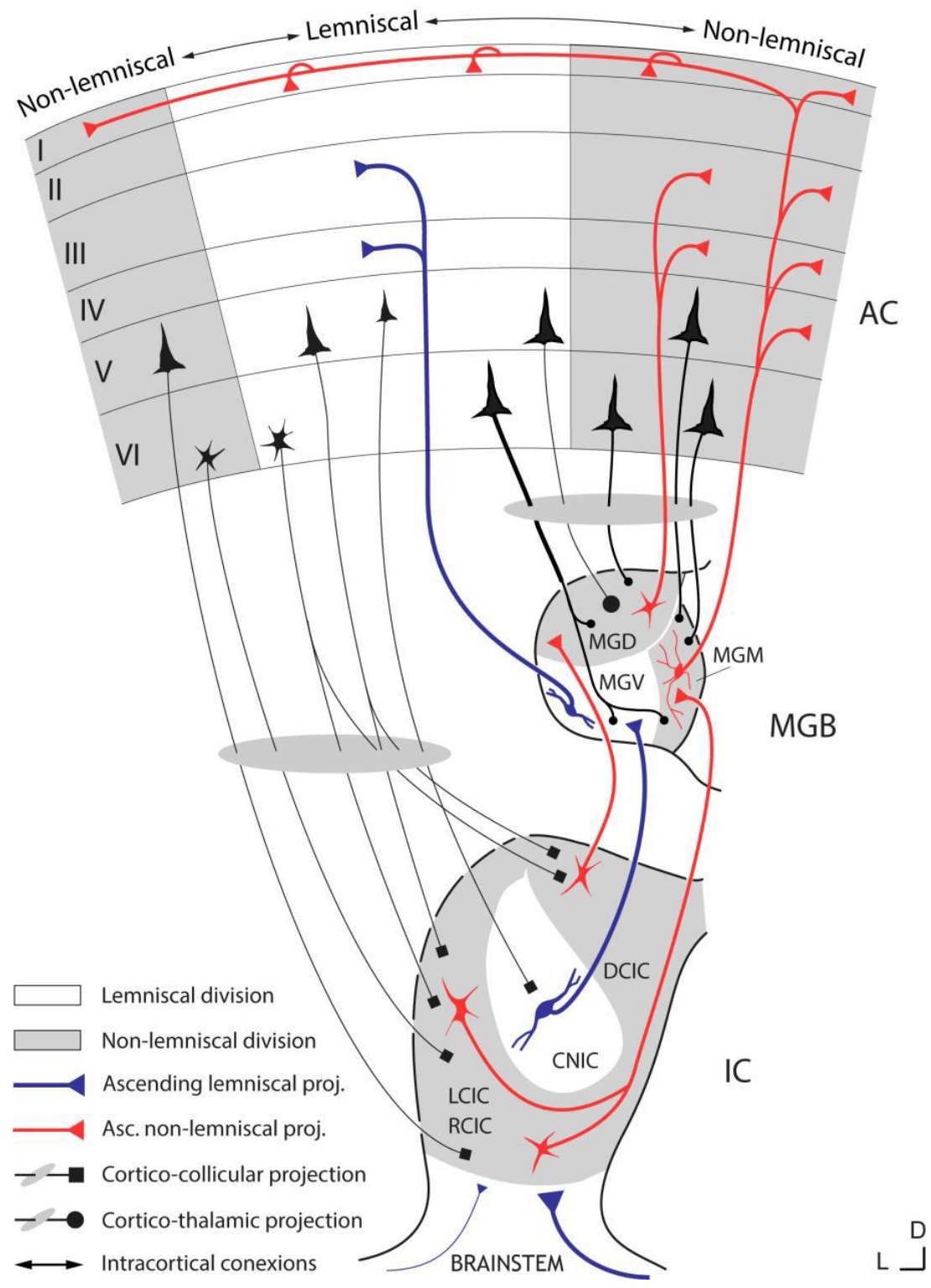

Fig 4. Schematic diagram of the ascending projection of lemniscal (blue) and non-lemniscal (red) pathways. Descending projections are colored in black. Reproduced from Malmierca (2015).

Stimulus-specific adaptation in the auditory system

In the central nervous system, there are fundamentally two types of adaptive neural responses when we present repetitive stimuli: one corresponds to the phenomenon of neuronal habituation, where there is a decrease in the rate of generalized neuronal discharge, and where the neuron does not immediately recover when presenting a train of stimuli (Pérez-González and Malmierca, 
2014). The other corresponds to specific-stimulus adaptation (SSA), which occurs when neurons decrease the response to frequently presented stimuli (standard) but not to rare stimuli (deviant). (Calford and Aitkin, 1983).

SSA is widespread and robust in the auditory midbrain, thalamus and cortex (Carbajal and Malmierca, 2018; Carbajal and Malmierca, 2020). Ulanovsky et al., (2003) first described SSA in the rat primary auditory cortex, using an oddball paradigm (Ulanovsky et al., 2003). Their study demonstrated for the first time the presence of SSA in the auditory cortex and proposed that SSA was an emerging property of the auditory cortex, since they failed to find SSA in MGB neurons. However, subsequent studies showed that this type of neuronal adaptation is also present in subcortical areas. In neuroanatomical terms, the midbrain is the first station where SSA is generated, particularly in the inferior colliculus (Malmierca et al., 2009; Pérez-González et al., 2005). The IC is the auditory center in the midbrain where nearly all ascending pathways converge before sending information to the AC via the thalamus (Antunes et al., 2010). Subsequently, it was shown that SSA was also present in the MGB, more specifically, along the non-lemniscal divisions. The later study contradicted that of Ulanovsky et al., (2003) which, as mentioned above, did not report SSA in MGB recordings. The most likely explanation for this is that Ulanovsky's recordings were restricted to the lemniscal areas of MGB. Moreover, the SSA is even maintained when the auditory cortex is deactivated by cortical cooling in both IC and MGB (Anderson and Malmierca, 2013; Antunes and Malmierca, 2011). On the other hand, SSA is dependent on different variables such as the intensity of the stimulus (lower sound intensity > higher SSA), frequency (high frequency stimuli generate a 
greater SSA) and the interval between stimuli (short separation between stimuli > higher SSA) (Antunes et al., 2010; Duque et al., 2012). All this, shows us that this phenomenon also occurs in subcortical structures and that it would be a common element throughout the entire auditory pathway, starting from the IC (Ayala et al., 2012).

In the auditory cortex, SSA is widely distributed and ubiquitous in both lemniscal and non-lemniscal areas. On the other hand, one can observe great differences between the neuronal responses coming from lemniscal and non-lemniscal fields. Nieto-Diego and Malmierca demonstrated that SSA levels are higher in nonlemniscal zones in the rat auditory cortex (SRAF and PAF), compared to lemniscal (A1, AAF, VAF), creating a topographic gradient along the auditory cortex. Moreover, the highest levels of SSA were obtained mainly at high frequencies and low intensities; this relationship being higher in lemniscal zones (Nieto-Diego and Malmierca, 2016). These findings have been confirmed in another study (Parras et al., 2017), where it has also been seen that the levels of error prediction are higher in non-lemniscal areas (Fig. 5). 


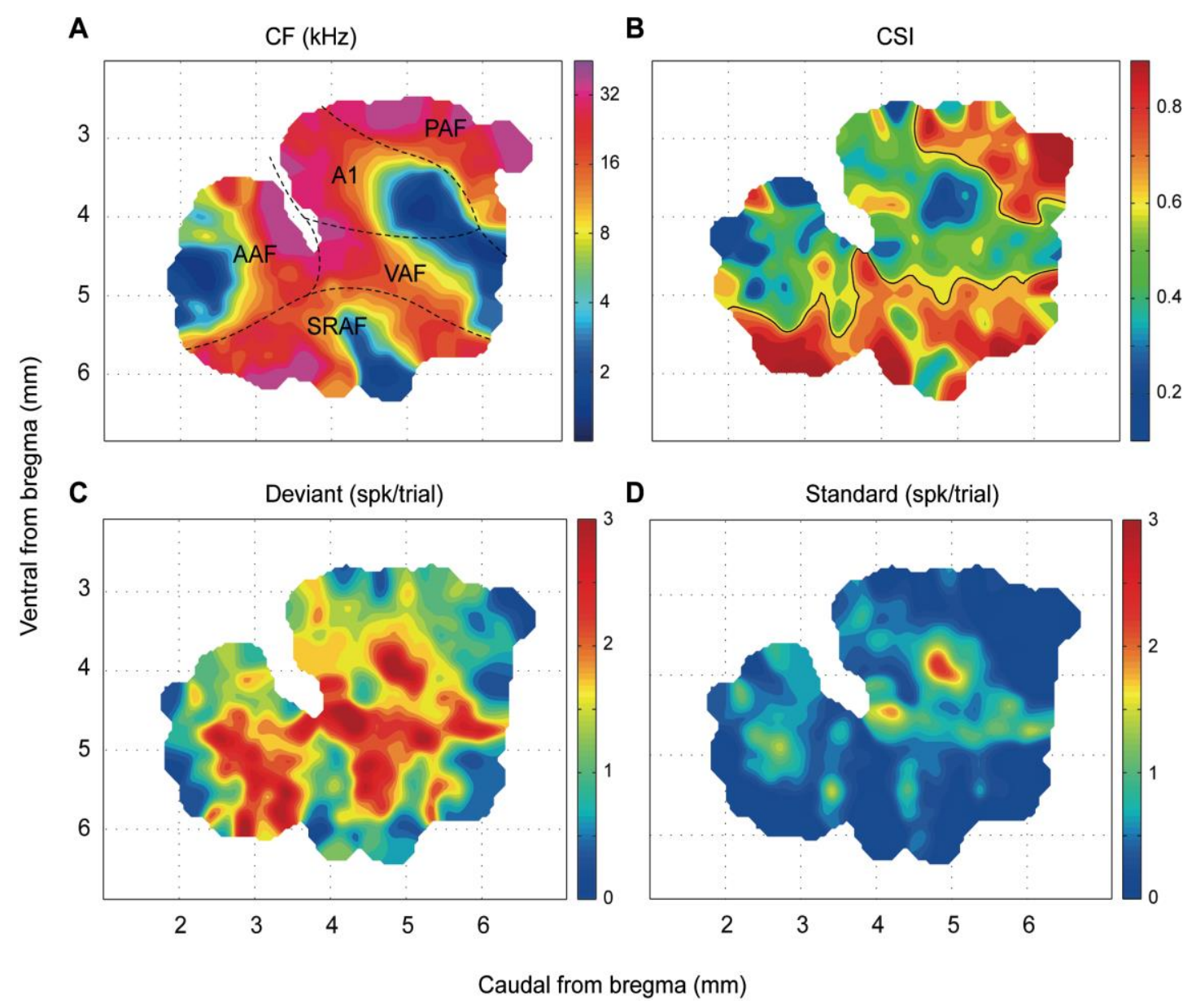

Fig 5. Topographic distribution of SSA throughout the auditory cortex. (A). Synthetic map of the auditory cortex showing the location of the five cortical fields. The characteristic frequency was used as the main reference to put into register the individual maps from the 12 animals. (B). Topographic distribution of SSA in the auditory cortex. The CSI follows a statistically significant topography within the auditory cortex, with the highest values being confined to the non-lemniscal fields. (C-D). Topographic distribution of the responses to deviant and standard tones, respectively, from which the CSI was computed. Responses to standard tones were almost zero in the non-lemniscal fields. Reproduced from (Nieto-Diego and Malmierca, 2016).

\section{Mismatch negativity}

Mismatch negativity (MMN) is an auditory event-related potential that occurs when a sequence of repetitive sounds is interrupted by an occasional "oddball" sound that differs in frequency or any other physical attribute. (Näätänen et al., 1978). More recently, MMN is best explained under the predictive coding framework (Friston, 2005) as a brain response to violations of a rule, established 
by a sequence of sensory stimuli (Garrido et al., 2009b). MMN corresponds to a negative wave sensory evoked response with a latency between 100-250 ms. We can obtain this response using a stimulation "oddball" paradigm (Fig. 6A) which consists of a series of repetitive stimuli with similar acoustic characteristics (standard stimulus) alternated randomly with discrepant acoustic stimuli (deviant stimulus) that differ from the first in any of its attributes such as frequency, intensity or duration (Näätänen et al., 2007). This paradigm generates auditory stimuli that appear with a high probability of occurrence (generally $90 \%$ for the standard stimuli) and others with a low probability (generally $10 \%$, for the deviant stimuli) (Fig. 6B).

A

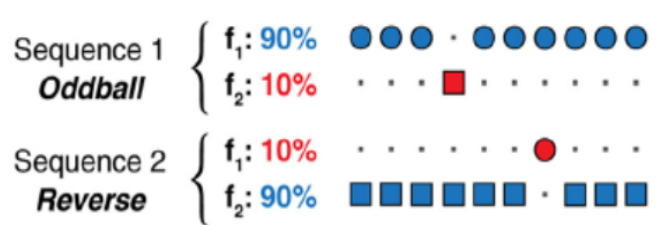

B

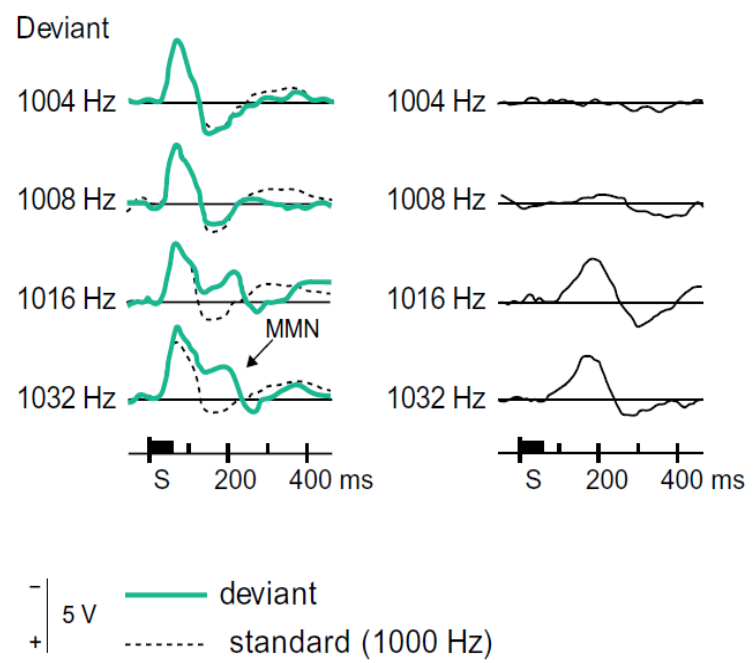

Fig 6. (A). Auditory sequences used in an oddball paradigm. Reproduced and modified from Duque et al. (2012). (B). Scheme of an MMN record obtained with different frequencies for the deviant tone. As the frequency of the deviant tone becomes more distant from the standard tone, the amplitude of the MMN is greater. Reproduced from: The mismatch negativity $(M M N)$ in basic research of central auditory processing: $A$ review (Näätänen et al., 2007)

Classically the analysis of the MMN has been thoughtfully studied using different variations of the oddball paradigm, primarily in the auditory domain. But MMN has also been obtained in other sensory systems such as the visual or somatosensory 
systems (Kremláček et al., 2016; Näätänen, 2009; Shen et al., 2018; Stefanics et al., 2015, 2014).

Näätänen and colleagues showed that MMN reveals computational properties of the auditory system to represent the regularity of the auditory scene (Näätänen et al., 2001). For this reason, the MMN demonstrates that in the auditory system, the neural mechanisms of sound pattern recognition would be revealed based on regular acoustic stimulation paradigms. This can be considered a form of "primitive intelligence" (Näätänen et al., 2001). The MMN would then result from the difference, or mismatch, between the current and preceding input. Näätänen and colleagues suggested that the MMN results from a comparison between the present auditory input and the memory trace of previous sounds (Näätänen, 2018). In agreement with this theory, others authors (Näätänen and Winkler, 1999; Sussman and Winkler, 2001; Winkler et al., 1996) have postulated that the MMN could reflect on-line modifications of a perceptual model that is updated when the auditory input does not match its predictions. This is the so-called model-adjustment hypothesis. In the context of the model adjustment hypothesis, the MMN results from a comparison between the auditory input and a memory trace of previous sounds, reflecting an on-line updating of the model for predicting auditory inputs (Garrido et al., 2009b). This interpretation has been traditionally favored by the MMN literature, which usually refers to this enhancement as genuine deviance detection. On the other hand, the contrast between deviant and standard stimuli could be simply due to attenuation of the response to the repetitive sound, as an effect of mere neuronal adaptation. The deviant sound would not produce an enhanced response, but just a non-adapted one. This 
interpretation conforms to the adaptation hypothesis (May et al., 1999). Although this idea is favored by many neurophysiological studies about SSA, there is evidence that does not allow the adaptation hypothesis to explain the generation of the MMN per se. For example, the MMN can be generated with infrequent changes in sound intensity (or the complete omission of the stimulus), that is, that a new stimulus is not necessary for the generation of the response (Yabe et al., 1997). Some authors have argued that the omission could yield an abrupt release of adaptation that would provoke a rebound of neuronal activity, confounding that activity recorded in the scalp with a genuine response (May et al., 1999). In the last fifteen years has emerged another explanation for MMN phenomenology: the predictive coding theory (Friston, 2005) which will be discussed in another section of this introduction.

The relationship between stimulus-specific adaptation and mismatch negativity It has been postulated that SSA could be the neural correlate of MMN, since they share many similarities (Ulanovsky et al., 2003, Fig. 7). In both cases, the use of an oddball paradigm triggers an increase in amplitude in the neuronal response to the unexpected tone. On the other hand, the magnitude of the MMN and the SSA are positively correlated with the magnitude of the difference between the discrepant and the standard stimulus. Furthermore, the amplitude of the MMN and SSA increases over a few repetitions, although it decreases with increasing inter-stimulus interval and the latency of both decreases when the difference between standard and deviant tones is increased. 

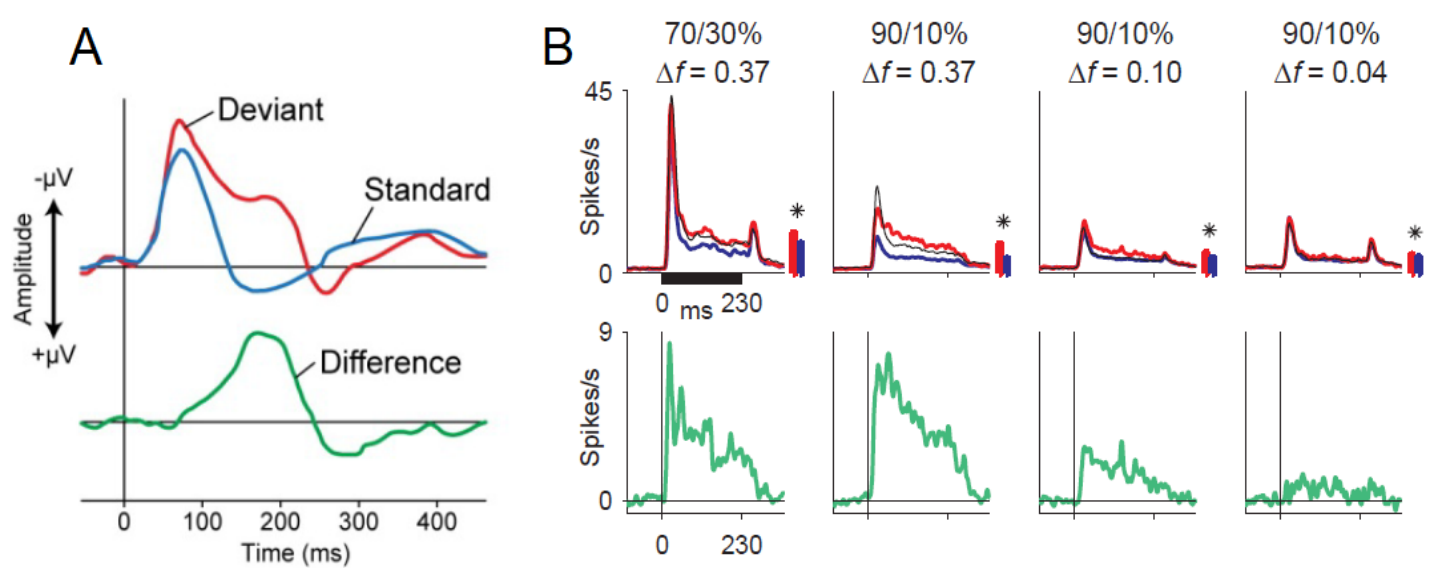

Figure 7. MMN and SSA examples. (A) MMN representation from human scalp-recording. (B) SSA representation for single neurons recording in the rat IC. Both cases represent the recorded signal during an oddball sequence for standard and deviant stimuli, as well as their difference (deviant-standard). Reproduced from (Ayala et al., 2012) and (Ulanovsky et al., 2003).

However, there are also some differences between the two processes. One of the most important is that the latency obtained in the MMN in $\mathrm{A} 1$ is longer than that found in the SSA (there is a difference of $50-100$ ms between the two).

The MMN would have secondary cortical areas as neural generators, while SSA was initially described in the primary auditory cortex (Ulanovsky et al., 2003). Nieto-Diego and Malmierca 2016, suggested that SSA is also generated in the rat secondary cortex. In their work, in all cortical fields, SSA is correlated in time and strength with the difference wave seen in both the fast $(\mathrm{Nd})$ and slower $(\mathrm{Pd})$ deflections of the local field potential (LFP); however, SSA is very strong in nonprimary auditory cortex, where are postulated to occur the main generators of the MMN in humans. This finding suggests that the main generators of MMN in humans would be found in non-primary auditory areas. This work also showed strong SSA levels in SRAF and PAF. The latency of this response was in the range of 50-100 ms and was correlated with a consistent difference wave at the slower positive deflection of the local field potential. The latency of this slower 
positive deflection (60-80 ms) is considerably shorter than the human MMN (150-200 ms) but matches perfectly the range of MMN-like potentials in the rat (Shiramatsu et al., 2013), which tend to occur, on average, 50-100 ms after stimulus onset, probably due to the smaller size of the rat brain (Nieto-Diego and Malmierca, 2016). Due to the aforementioned, the secondary auditory cortex would be a strong candidate to be the neural generator of MMN.

\section{Predictive coding framework}

In recent years, the predictive coding has gained interest. According to the predictive coding theory, perception emerges from integrating sensory information from the environment and our predictions based on an internal representation of that information (Friston, 2010, 2005). Thus, higher-level cortical areas generate predictions about the environment that are sent in a topdown manner to lower hierarchical levels, to suppress the ascending neuronal activity evoked by sensory events that can be anticipated. However, when current predictions do not match the actual sensory inputs, then the lower levels will send forward bottom-up prediction errors to higher hierarchical levels (Friston, 2008; Friston and Kiebel, 2009). The current inputs are predicted from past events through a model, and the finality of the system is to minimize errors in the prediction by continuously updating the model. The reduction of prediction error is achieved through recurrent interactions among levels of a processing hierarchy, organized in distinct anatomical structures and neuronal populations (Bastos et al., 2012a) (Fig.8). Computational models weigh prediction errors of sensory input by their precision, which is the inverse of sensory variance, as prediction errors can exist in varying levels of uncertainty (Parr and Friston, 
2017). In neurobiological terms, precision is mediated by the action of the neuromodulatory systems, including the dopaminergic (Valdés-Baizabal et al., 2020a), or the cholinergic system (Ayala and Malmierca, 2015; Moran et al., 2013). The predictive coding theory would explain the phenomenon of attenuation of the neuronal response when a stimulus is repetitive (repetition suppression) or a strong increase in the response when the sensory input is novel versus expected (prediction error) (Auksztulewicz and Friston, 2016). The predictive coding theory assumes a hierarchy of sensory processing levels, where the outputs of one level would inform the other levels in order to update the internal model. 


\section{Canonical microcircuit}

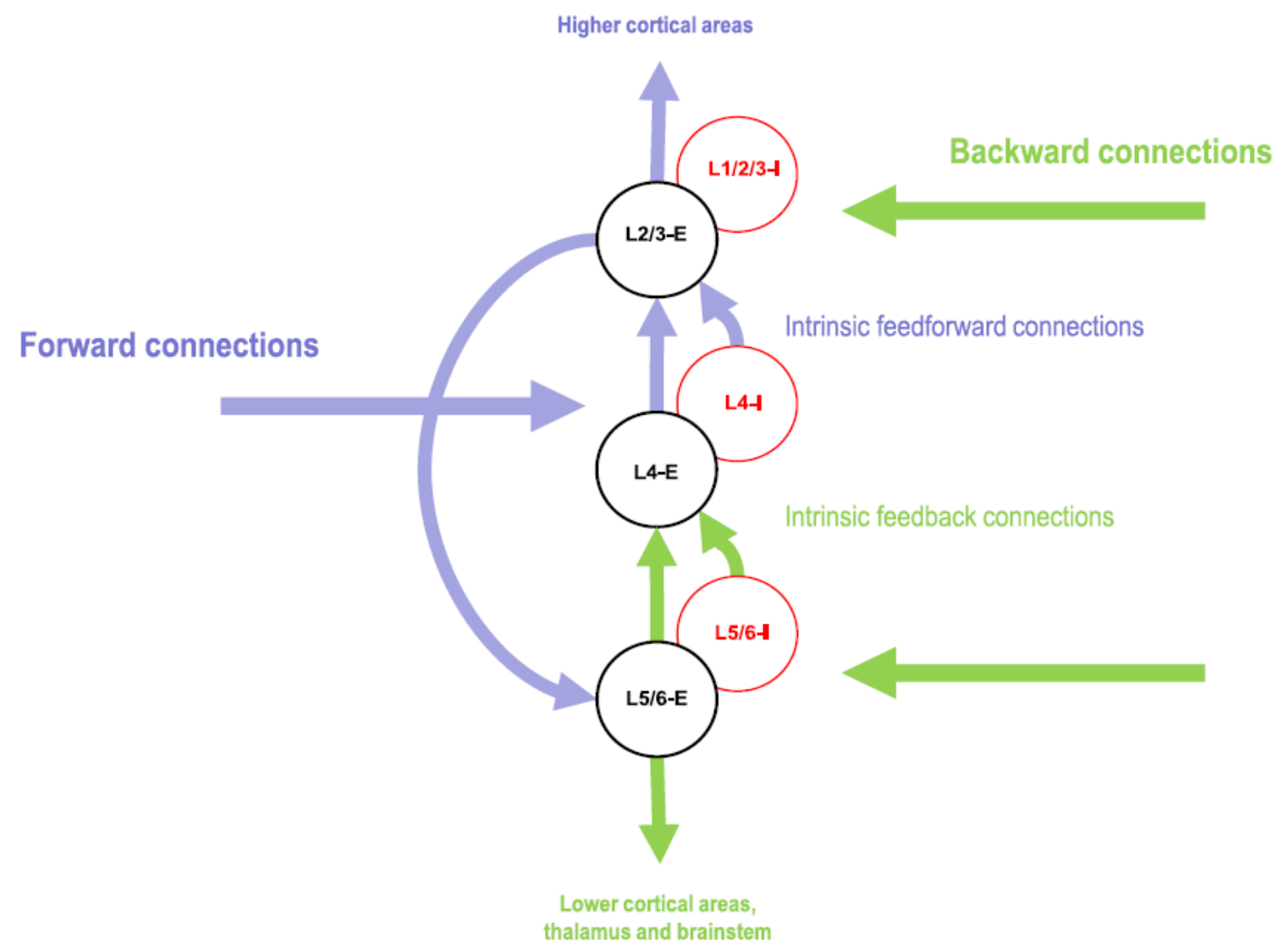

Figure 8. The Canonical Cortical Microcircuit in the predictive coding framework. This is a simplified schematic of the key intrinsic connections among excitatory (E) and inhibitory (I) populations in granular (L4), supragranular (L1/2/3), and infragranular (L5/6) layers. Forward connections denote feedforward extrinsic corticocortical or thalamocortical afferents that are reciprocated by backward or feedback connections. Anatomical and functional data suggest that afferent input enters primarily into $L 4$ and is conveyed to superficial layers $L 2 / 3$ that are rich in pyramidal cells, which project forward to the next cortical area, forming a disynaptic route between thalamus and secondary cortical areas. Information from L2/3 is then sent to $L 5$ and $L 6$, which sends feedback projections back to $L 4$. $L 5$ cells originate feedback connections to earlier cortical areas. In summary, forward input is segregated by intrinsic connections into a superficial forward stream and a deep backward stream. Reproduced from (Bastos et al., 2012b). 


\section{Acetylcholine}

Acetylcholine (ACh) is a neurotransmitter widely distributed in the central nervous system and the first to be described by Henry Dale and Otto Loewy (For this achievement, they won the Nobel Prize in Physiology in 1936) (Loewi, 1921). It is synthesized from serum choline and is made up of two components, acetate and choline, which are united by the action of acetylcholine transferase. At the cholinergic terminals, the neurotransmitter is synthesized in the cytoplasm, where it is released into the synaptic space, or it is transported into synaptic vesicles, through an exocytosis process. Acetylcholine released into the synaptic space acts on its receptors, or it can be hydrolyzed by the action of acetylcholinesterase (Picciotto et al., 2012). There are two main types of cholinergic receptors: nicotinic and muscarinic. Nicotinic receptors allow the opening of ion channels (receptor ionotropic type), which have a binding site for the neurotransmitter and contain the ion channel that allows the signal to be transmitted into the cell. Muscarinic receptors, on the other hand, are mediated by interaction with type $G$ proteins (nucleotide binding proteins of guanine), using cyclic GMP as the second messenger. This type of receptor is known as metabotropic, which is slightly slower in its response (Jones et al., 2012) (Fig. 9). 


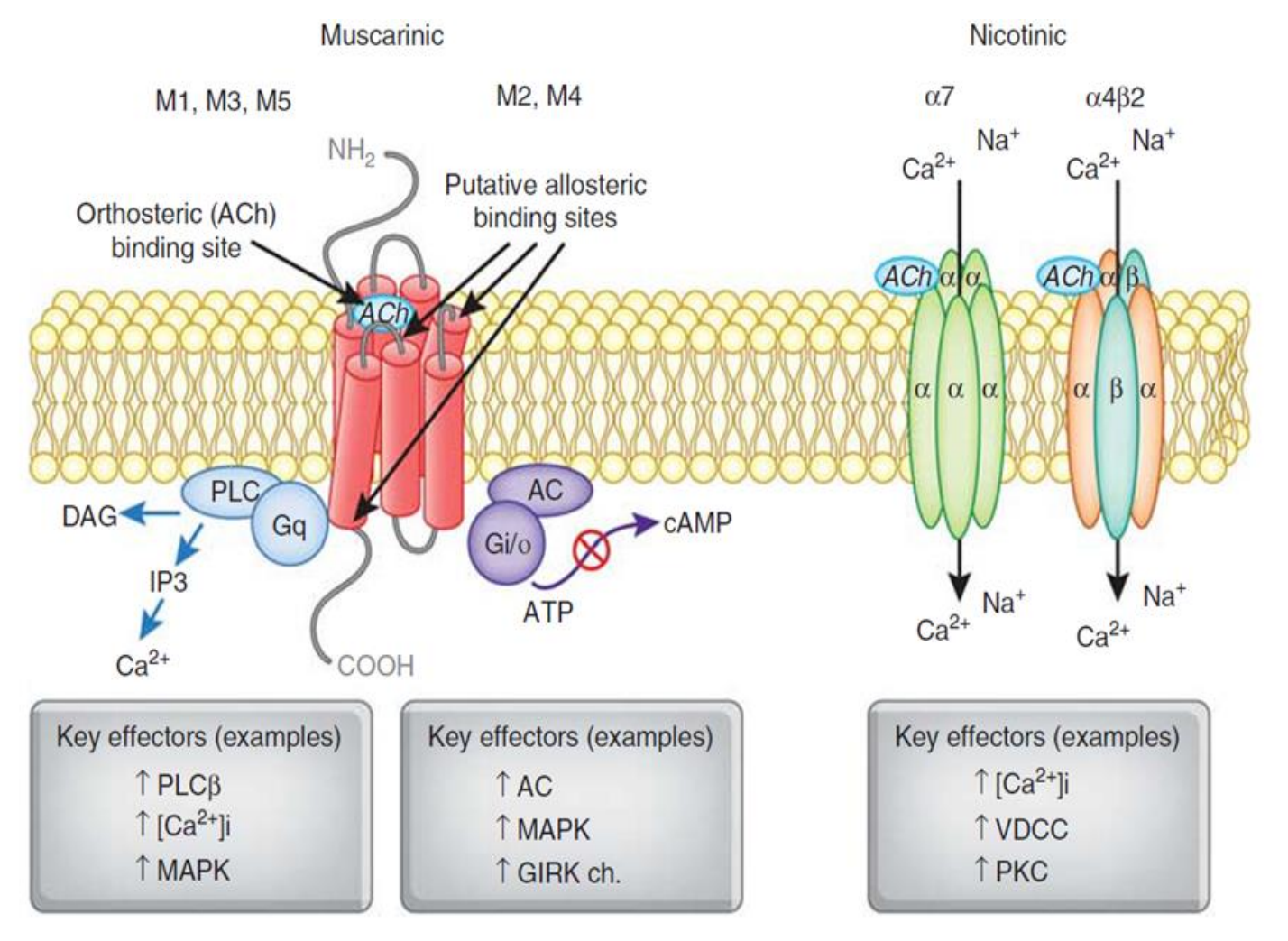

Figure 9:The structure and signaling pathways of $\mathrm{mAChRs}$ and $\mathrm{nAChRs}$. Each mAChR subtype is a seven-transmembrane protein, which belongs to two major functional classes based on G-protein coupling. The M1, M3, and M5 mAChRs selectively couple to the $G$ /G11-type G-proteins resulting in the generation of inositol-1,4,5-trisphosphate (IP3) and 1,2-diacylglycerol (DAG).The M2 and M4 mAChRs preferentially activate Gi/Go-type G-proteins, thereby inhibiting adenylate cyclase, reducing intracellular concentration of cAMP, and prolonging potassium channel opening.

Neuronal nAChRs are pentameric ligand-gated ion channels. The most abundant neuronal subunits are a4, b2, and a7, with the heteromeric a4b2 receptor subtype in highest abundance. The heteromeric a4b2 receptor subtype can exist in two different forms: (a4)2(b2)3 receptors show low $\mathrm{Ca} 2+$ permeability and high affinity to ACh and nicotine, whereas (a4)3(b2)2 receptors have high Ca2+ permeability. By contrast, the a7 nAChR also shows high permeability to $\mathrm{Ca} 2+$ relative to the heteromeric a4b2 nAChRs. The action of a4b2 nAChRs can enhance intracellular levels of $\mathrm{Ca} 2+$ by secondary activation of voltage-operated calcium channels (VOOCs), whereas a7 nAChRs preferentially increase $\mathrm{Ca} 2+$ release from ryanodine-sensitive intercellular stores through $\mathrm{Ca} 2+$-induced $\mathrm{Ca} 2+$ release (CICR). The capacity of these different nAChR subtypes to couple to VOCCs or CICR mechanisms results in distinct patterns of $\mathrm{Ca} 2+$ signaling that can provide a broader control of synaptic plasticity and neurotransmitter release, as well as gene transcription. Reproduced from (Jones et al., 2012). 
Acetylcholine modulates different neurobiological processes such as attention, activation (arousal), learning, memory, sleep and cognitive reinforcement (Bentley et al., 2011; Hasselmo and Giocomo, 2006; Newman et al., 2012). Two main sources of $\mathrm{ACh}$ occur in the brain. ACh originating from the pedunculopontine and laterodorsal tegmental areas project to subcortical brain regions, while the main source of ACh in the auditory cortex is the basal forebrain (Mesulam, 2013; Villano et al., 2017) (Fig. 10).

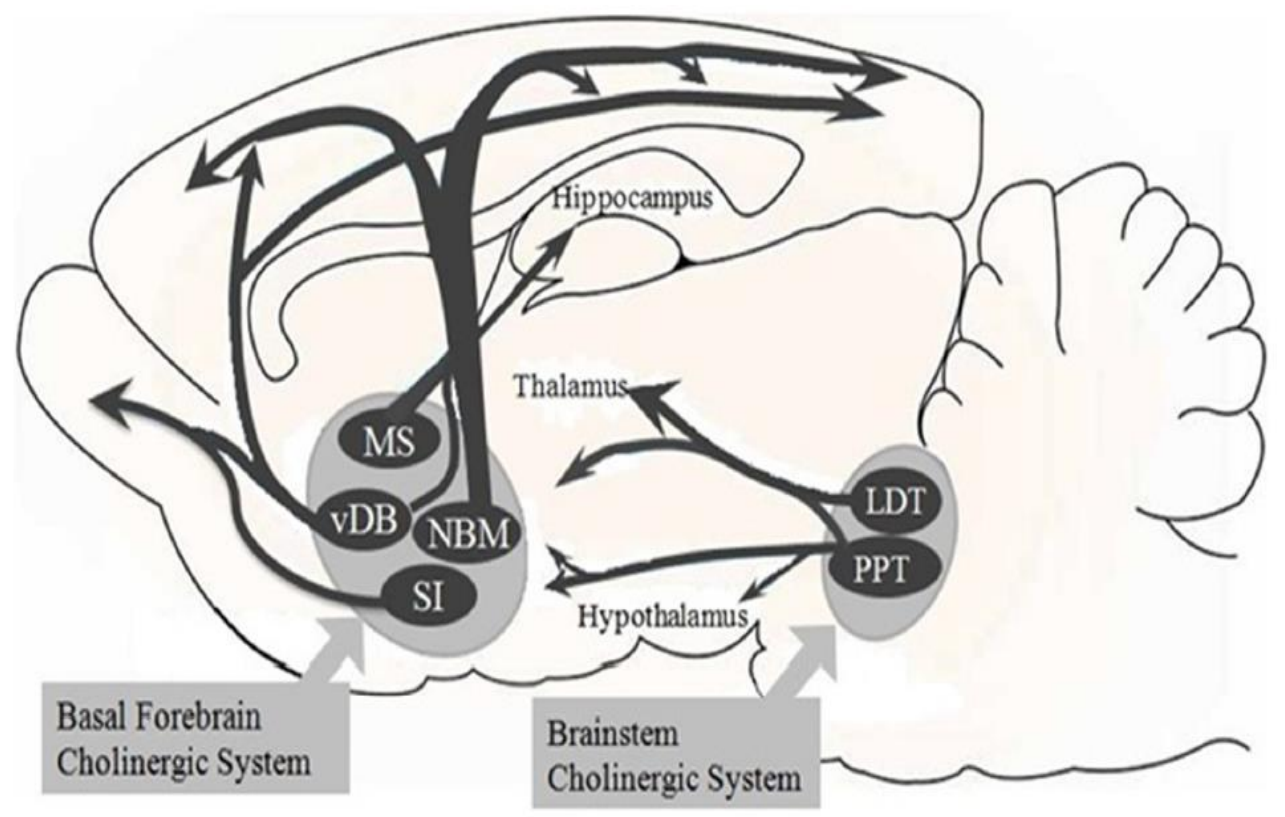

Fig 10. Overview of the basal forebrain (BF) cholinergic pathway. The BF cholinergic system includes the medial septum (MS), vertical limbs of the diagonal band of Broca (vDB), nucleus basalis of Meynert (NBM), and substantia innominate (SI). The VDB and NBM have diffuse projections to all parts of the neocortex and to basolateral amygdala and olfactory bulb. The MS and vDB project to hippocampus. Besides, the brainstem cholinergic system projects to the thalamus and hypothalamus but also to the BF region. This system includes the pedunculopontine tegmental nucleus (PPT) and laterodorsal pontine tegmentum (LDT), corresponding to brainstem cholinergic system. Reproduced from (Villano et al., 2017). 


\section{Acetylcholine and sensory systems}

ACh plays important roles in arousal, attention, and sensory learning (Hasselmo, 1999; Hasselmo and Sarter, 2011; Metherate, 2011; Picciotto et al., 2012; Sarter et al., 2001; Weinberger, 2004). The acetylcholine released in the mammalian basal forebrain promotes activation of muscarinic and nicotinic receptors in auditory, visual, and somatosensory cortices (Disney et al., 2007; Eggermann et al., 2014; Metherate, 2011). In the visual cortex, ACh modulates the gain control response (Soma et al., 2013) and induces the depolarization of pyramidal neurons by interneurons called vasoactive neurons for intestinal positive peptides $(\mathrm{VIP}+)$, which allows modulating targeting selectivity in neurons $\mathrm{V} 1$ in locomotortype tasks (Fu et al., 2014) while in the somatosensory cortex of mice (barrel cortex) acetylcholine by thalamo-cortical discharges allows the activity in the somatosensory cortex to be desynchronized during tasks of active detection of vibrissae (Eggermann et al., 2014). In the auditory cortex, cholinergic activation of VIP+ neurons can increase sensory processing gain modulation through disinhibitory circuits (Fu et al., 2014). Acetylcholine would optimize afferent responses when sensory input is received, decreasing intracortical processing (Metherate et al., 1992). In addition, electrical stimulation protocols in the anterior basal brain have shown to improve thalamo-cortical transmission by exciting cortical GABAergic interneurons through presynaptic receptors in the auditory system (Metherate et al., 1992). Moreover, cholinergic modulation modifies the coding of the spectral representation of cortical auditory neurons, generating changes by increasing the degree of tuning of the response and decreasing the acoustic threshold at the characteristic frequency and changes the coding of the spectral representation (Ma and Suga, 2005; Metherate, 2011). The ACh- 
mediated effects are produced by a rapid disinhibition of neuronal responses, modifying synaptic strength, enhancing excitatory-inhibitory balance and reorganizing cortical circuits promoting cortical plasticity (Froemke et al., 2007; Irvine, 2018a, 2018b; Metherate, 2011; Picciotto et al., 2012)

Acetylcholine and stimulus-specific adaptation

Previous studies conducted in the IC showed that ACh differentially increased the response to the standard tone (but not to the deviant) in an oddball paradigm. This effect results in a significant decrease in SSA and augmenting the repetition suppression levels. The use of cholinergic antagonists (scopolamine and mecamylamine) reversed these effects, but only scopolamine did so significantly (Ayala and Malmierca, 2015). Moreover, previous works suggested that ACh may encode changes in the precision of prediction errors in sensory cortical hierarchies (Friston, 2008) and modulate the optimizing precision by gain modulation in supragranular pyramidal cells in primary sensory cortex (Moran et al., 2013). 


\section{HYPOTHESIS}

Considering that 1) the central nervous system, and particularly the auditory system, is hierarchically organized, and 2) that according to the predictive coding framework, increasing prediction error levels occurs from the inferior colliculus to the auditory cortex and 3 ) that auditory cortex receives a strong cholinergic innervation, I propose the following hypothesis:

Acetylcholine modulates the levels of SSA and prediction error in the auditory cortex. Moreover, I also propose that acetylcholine plays a key role in the detection of saliency in behavioral relevant sounds. 


\section{OBJETIVES}

To test this general hypothesis, I present the following specific objectives:

1) Determine if SSA varies across neuron types in the auditory cortex.

2) Determine how acetylcholine affects SSA in the auditory cortex throughout the different layers of the auditory cortex.

3) Determine which of the acetylcholine receptors mediate SSA modulation.

4) Determine if acetylcholine modulates the prediction error and repetition suppression levels in the auditory cortex, and whether or not ACh plays a role in prediction error precision.

5) Determine if training plays a role is the saliency detection of behaviorally relevant sounds. 


\section{MATHERIAL AND METHODS}

For the experiments of the first section of this thesis (deviance detection in physiologically identified cell types in the rat auditory cortex), the recordings were performed in 86 urethane-anesthetized female Long Evans adult rats (body weight 200-250 g). The experiments in the second section (Acetylcholine modulates SSA and prediction error response in auditory rat cortex) were performed in 31 rats Long Evans adult rats (body weight 180-260 g) and finally, for the third section (Effect of behaviorally relevant sounds in deviant detection and prediction error responses) were used 20 female Long Evans rats (body weight 200-250 g).

\section{Experimental Design for electrophysiological recordings}

For the electrophysiological recording experiments, animals were anesthetized with urethane $(1.5 \mathrm{~g} / \mathrm{kg}$, intraperitoneal), with supplementary doses $(0.5 \mathrm{~g} / \mathrm{kg}$, intraperitoneal) given as needed. Dexamethasone $(0.25 \mathrm{mg} / \mathrm{kg})$ and atropine sulfate $(0.1 \mathrm{mg} / \mathrm{kg})$ were administered at the beginning of the surgery to reduce brain edema and the viscosity of bronchial secretions, respectively. Prior to surgery, normal hearing was verified with auditory brainstem responses (ABR) recorded with subcutaneous needle electrodes, using a RZ6 Multi I/O Processor (Tucker-Davis Technologies, TDT) and processed with BioSig software (TDT). ABR stimuli consisted of $100 \mu$ s clicks at a $21 / \mathrm{s}$ rate, delivered monaurally to the right ear in $10 \mathrm{~dB}$ steps, from 10 to 90 decibels of sound pressure level (dB SPL), using a closed-field speaker. After the animal reached a surgical plane of anesthesia, the trachea was cannulated for artificial ventilation and a cisternal drain was introduced to prevent brain hernia and brain edema. Isotonic 
glucosaline solution was administered periodically $(5-10 \mathrm{ml}$ every $7 \mathrm{~h}$, subcutaneous) throughout the experiment to prevent dehydration. Body temperature was monitored with a rectal probe thermometer and maintained between 37 and $38^{\circ} \mathrm{C}$ with a homoeothermic blanket system.

During surgery, the temporal bone was exposed and the auditory cortex was located using stereotactic coordinates (Paxinos et al., 1997). A craniotomy was performed over the auditory cortex, the dura was removed carefully, and the exposed area was filled with a layer of agar to prevent desiccation and to stabilize the recordings. Before applying the agar, a magnified picture $(25 x)$ of the exposed cortex was taken with a digital camera coupled to the surgical microscope (Zeiss) through a lens adapter (TTI Medical). The picture included a pair of reference points previously marked on the dorsal ridge of the temporal bone, indicating the absolute scale and position of the image with respect to bregma (the reference point). For the identification of the primary and secondary cortical areas, the reference template was used, used in other studies (Parras et al., 2017). These boundaries correspond to tonotopic gradients within the different fields, with a high frequency reversal between VAF and AAF (rostrally), a low-frequency reversal between A1 and PAF (dorsocaudally) and a highfrequency reversal between VAF and SRAF (ventrally). These boundaries were used to assign each recording to a given field (Nieto-Diego and Malmierca, 2016) (Fig.5). 


\section{Electrophysiological recording and microiontophoresis}

A tungsten electrode (1-3 M $\Omega$ ) was used to record multiunit neuronal activity. For the microiontophoresis experiments, it was attached to a 5-barrel multibarrel borosilicate glass pipette that carried drug solution to be delivered in the vicinity of the recorded neuron. The multibarrel's tip was cut to a diameter of about 20$30 \mu \mathrm{m}$ approximately. The center barrel was filled with saline solution for current compensation (165 $\mathrm{mM} \mathrm{NaCl}$ ) whereas the others barrels were filled with $1 \mathrm{M}$ acetylcholine chloride (Sigma, catalog no. A6625), 0.5 M scopolamine hydrobromide (Sigma, catalog no. S0929), or $0.5 \mathrm{M}$ mecamylamine hydrochloride (Tocris Bioscience, catalog no. 2843). The $\mathrm{pH}$ of acetylcholine, scopolamine and mecamylamine was adjusted between 4.0-4.2 (Ayala and Malmierca, 2015). Scopolamine and mecamylamine are antagonists of muscarinic and nicotinic receptors, respectively, whereas $\mathrm{ACh}$ is an agonist for both types of receptors. All drugs were retained by applying a $-15 \mathrm{nA}$ current, and were ejected when required, typically using 30-40 nA currents (Neurophore BH-2 System, Harvard Apparatus). The duration of the drug ejection usually lasted 8-10 min and the recording protocols were extended until the effect of the drug had disappeared (60 to 90 minutes approximately). The multibarrel assembly was positioned over the pial surface of the auditory cortex, forming a $30^{\circ}$ angle with the horizontal plane towards the rostral direction, and advanced using a piezoelectric micromanipulator (Sensapex $\AA$ ) until we observed a strong spiking activity synchronized with the train of search stimuli. Analog signals were digitalized with

a RZ6 Multi I/O Processor, a RA16PA Medusa Preamplifier and a ZC16 headstage (TDT) at $12 \mathrm{kHz}$ sampling rate and amplified 251x. Neurophysiological signals for multiunit activity were band-pass filtered between 0.5 and $4.5 \mathrm{kHz}$. 


\section{Experimental Design and stimulation paradigms}

Sound stimuli were generated using the RZ6 Multi I/O Processor (TDT) and custom software programmed with the OpenEx Suite (TDT) and MATLAB. Sounds were presented monaurally through a speaker, in a close-field condition to the ear contralateral to the left auditory cortex. The experimental stimuli were pure tones in the range $0.5-44 \mathrm{kHz}$, with a duration of $75 \mathrm{~ms}$, including $5 \mathrm{~ms}$ rise/fall ramps presented at a rate of 4 stimuli/s. Once a suitable neuron was found, the frequency response area (FRA) of the cell, i.e. the combination of frequencies and intensities capable of evoking a suprathreshold response, was obtained automatically using a randomized paradigm that presented tones between $0.5-44 \mathrm{kHz}$ in 25 logarithmic steps, with intensities spaced by $10 \mathrm{~dB}$ steps, from 0 to $70 \mathrm{~dB} \mathrm{SPL}$, at a repetition rate of $4 / \mathrm{s}$ (Fig. 18). Based on this information, we selected a pair of frequencies evoking similar responses at 10$30 \mathrm{~dB}$ above threshold.

\section{Oddball Sequences}

We used pure tones at these frequencies as the stimuli in the oddball paradigm. Oddball sequences consisted of frequently repeating stimuli (standard tones) which were pseudo-randomly interleaved with rare events (deviant tones). Two oddball sequences with fixed parameters (400 trials each, 75 ms stimulus duration, 0.5 octaves frequency separation, 10\% deviant probability, $250 \mathrm{~ms}$ onset to onset, and a minimum of three standard tones before a deviant) were presented for every pair of stimuli thus selected. In one of the sequences, the low frequency (f1) was the "standard" and the high frequency (f2) was the "deviant," and in the other sequence their roles were inverted (Fig. 6). The order of 
presentation of these two sequences was randomized across sites. In some cases, one or more extra pairs of stimuli were selected, and the oddball sequences were repeated with the new stimuli.

\section{Many-Standard and cascade sequences}

The mismatch responses like those obtained during an oddball paradigm can be divided in two components: repetition suppression (RS), a reduction in the response caused by a repeated stimulus, and prediction error (PE), an increased response caused by the violation of a regularity (Carbajal and Malmierca, 2018). In a subset of the experiments, we used control sequences to evaluate the separate contribution of RS and PE. Control sequences consisted of 10 tones evenly spaced by 0.5 octaves (same as in the oddball sequences), including the tones used in the oddball paradigm, and all stimuli at the same previously chosen sound level. Each control sequence lasted 400 trials, the duration of all stimuli was $75 \mathrm{~ms}$ and the presentation rate $4 / \mathrm{s}$. We used two different control sequences, namely the many-standard and cascade sequences (Fig. 11). The many-standards control is the consecutive presentation of blocks of 10 tones randomly ordered within the block, each tone with a $10 \%$ probability of occurrence (Schröger and Wolff, 1996). In this sequence the tones are unpredictable, and it is not possible to establish a rule which could be used to predict the following tones. On the other hand, the cascade control consists of the regular presentation of the same 10 tones in ascending or descending frequency succession (Ruhnau et al., 2012). This sequence also avoids the effects of the repetition of a single stimulus, but in this case, it maintains a predictable context (Parras et al., 2017). 
EXPERIMENTAL CONDITIONS

A
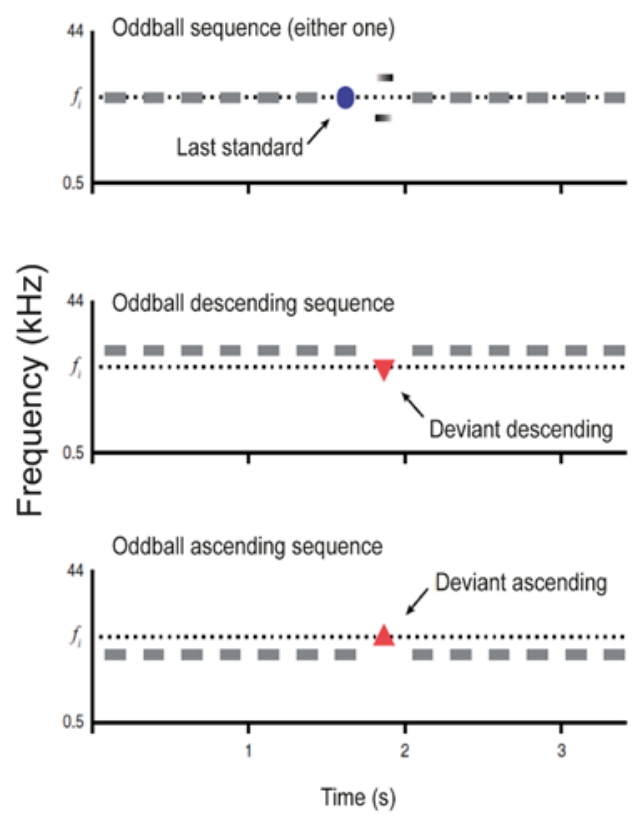

CONTROL CONDITIONS

$\mathrm{B}$
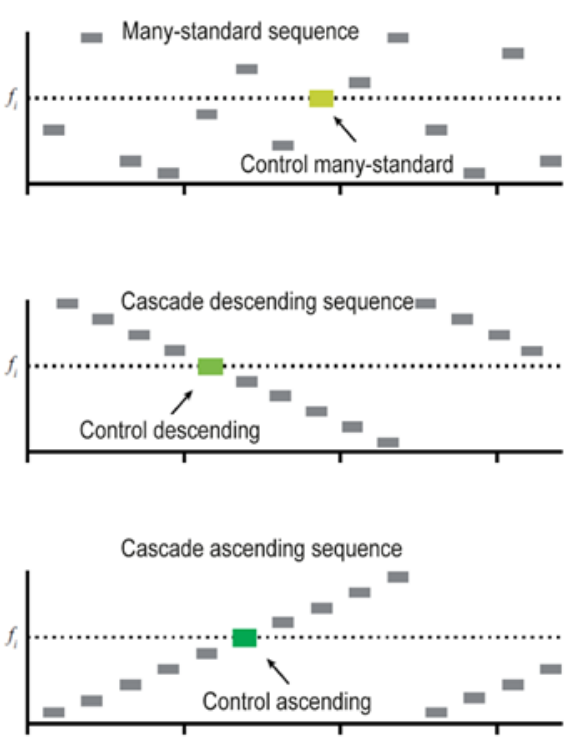

\section{PREDICTIVE CODING HYPOTHESIS}

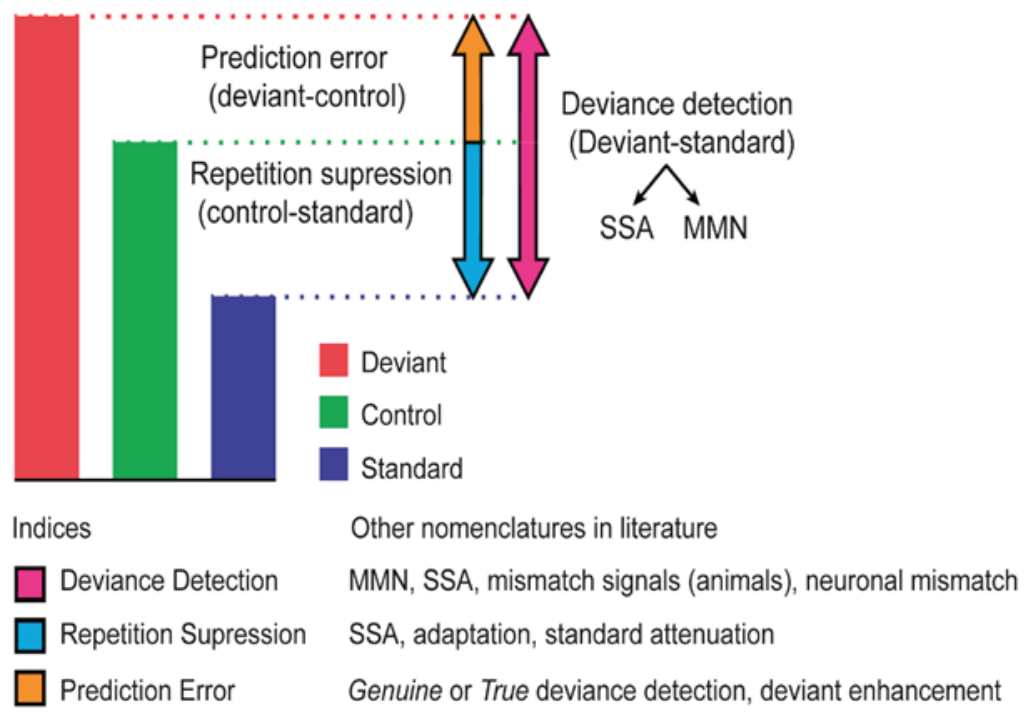

Fig 11. (A) Experimental conditions. Classical oddball paradigm, displaying three possible experimental conditions for a given target tone. We presented a 400 stimuli sequence containing deviant and standard frequencies in a probabilistic manner. One frequency $(f 1)$ was presented as the standard (high probability, $90 \%)$; and the second frequency $(f 2)$ was presented as the deviant stimuli (low probability, 10\%). After obtaining one data set, the relative probabilities of the two stimuli were reversed, with $f 2$ as the standard and $f 2$ as the deviant. (B) Control conditions. In the many-standards sequence, the target tone is embedded within a random succession of equiprobable tones, which prevents the system from generating a predictive rule. In the cascade 
sequences (ascending and descending), the target tone is embedded in a predictable succession of equiprobable tones, that is not broken by the appearance of the target tone. (C) Decomposition of deviant, control and standard responses according to predictive coding. The difference between the deviant and standard signal (DEV-STD) corresponds to the deviance detection, while the difference between deviant and control activity (DEV-CTR) corresponds to the prediction error. Finally, the difference between the response to the target tone in the control sequence and its evoked response when presented as a standard in the oddball sequence (CTR-STD) would constitute the component of repetition suppression. Adapted from (Parras et al., 2017b).

Experimental Design and stimulation paradigms for behavioral experiments

Behavioral box

All behavioral experiments were carried on a Med Associates operational cage

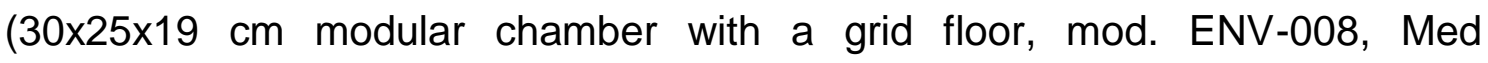
Associates, Inc., Georgia, USA), controlled by custom-made scripts (written in Trans V software by Cibertec, S.A., Madrid, Spain, and customized by us) with an interface smart controller (DIG-716P2). All devices installed in the box were purchased to Med Associates, Inc. The operational cage was equipped with a house light (ENV-215M) which was turned off before starting and turned on at the end of every session, used as a signal for the animal. Light was located in the superior-central zone of the left panel of the cage. The speaker (ENV-224BM), positioned in the superior-back zone of the left panel, was controlled by a sound generator (ANL-926). Calibration of the speaker was made using a 1/4-inch condenser microphone (model 4136, Brüel \& Kjaer) and a dynamic signal analyzer (Photon+, Brüel\&Kjaer). Rats were required to respond with a nose poke in a port (ENV-114BM) installed next to a tray for rewards in the right panel of the cage. The rewards were $45 \mathrm{mg}$ dustless precision pellets (Bilaney Consultants, 
Düsseldorf, Germany) dispensed by a modular pellet dispenser (ENV-203M) connected with the tray for rewards in the right side of the cage (Fig. 24B). These responses were automatically quantified by software MED-PC V version 5.1 (Med Associates, Inc.). All sessions were recorded using a HD LED IR cam (ELP Ailipu Technology Co., Ltd, Shenzhen, Guangdong, China) placed to capture a cenital view.

\section{Shaping protocol}

To evaluate the ability of the rats to detect and discriminate a sound embedded in a given auditory environment, we developed a behavioral go/no-go operant conditioning auditory discrimination task.

Stage 1 ( 7 sessions): Rats were familiarized to handling (2 sessions per day) for one week (Fig. 24A). Stage 1 consisted in $30 \mathrm{~min}$ of habituation to the experimental room followed by $30 \mathrm{~min}$ of handling. Mean weight during those 3 days was stablished as the initial weigh (measured at the end of the session). Then, we started food restriction of animals with a total privation over one night, and their weight was maintained between $90-95 \%$ of the initial weight until the end of experiment.

Stage 2 ( 3 sessions): Following $30 \mathrm{~min}$ of handling, rats were taken into an operational cage. In order to get over neophobias, 5 rewarding pellets were in the cage at the beginning of the session, and 3 unexpected pure tones were randomly presented with a speaker $(70 \mathrm{~dB}$, duration $400 \mathrm{~ms}$, risefall $10 \mathrm{~ms})$. The frequency used in this stage was fixed per animal and corresponded to the one 
used next as a deviant tone in the oddball paradigm; see below). Four groups of 4 animals were stablished (Table 2) according to the pair of tone frequencies presented during the last stage of training. Specifically, tone frequencies used here were 6.7 kHz for Group 1, 8.0 kHz for Group 2, $9.5 \mathrm{kHz}$ for Group 3 and 11.3 $\mathrm{kHz}$ for Group 4. Other 5 pellets were delivered at the end of these sessions.

Stage 3 (3-5 sessions): Next, rats had to learn the association between the activation of a nose-poke after a tone (the same as before) and reward delivery (Fig. 24A, Nose-poke shaping). Once the animal was taken into the cage, the program was initiated after $1 \mathrm{~min}$, and first tone was presented $1 \mathrm{~s}$ later. At the beginning of this stage, rats activated the nose-poke unintentionally, obtaining one pellet. There was not a maximum response time following a tone to facilitate the association. Next, every stimulus was presented $1 \mathrm{~s}$ after each nose-poke activation. Animals took around 3 sessions to get the maximum number of rewards (15) in less than 30 min.

Stage 4 (9 sessions): Then, we applied a protocol where rats had to go to the port after listening to a tone (the same as before) that is presented recurrently every $2.5 \mathrm{~s}$. The response window for poking the central port was $2.5 \mathrm{~s}$ (Fig. 24A, Sound shaping). In the following sessions, we progressively reduced the response window (to $1.5 \mathrm{~s}$ ) while increasing the silence periods (to $4.5 \mathrm{~s}$ ).

Stage 5 (10-15 sessions): The last stage of the training protocol consisted in a classical oddball paradigm (Fig. 24A, Oddball training; Fig. 25A), where rats learned to identify deviants ( $10 \%$, low probability), tones that will be rewarded) 
randomly intermingled in a sequence of regular standard ( $90 \%$, high probability) tones that were not be rewarded. Possible responses (Fig. 24B) were quantified as "hits" (nose-poke responses in the response window after a deviant tone rewarded with one pellet; HIT), "false alarms" (responses to a standard tone; FA), "correct rejections" (absence of response after a standard tone; CR) and "missed responses" (absence of response to deviant tones; MISS). Each FA was punished with $5 \mathrm{~s}$ of timeout, counting nose-poke responses during timeouts (TOR) as stress measurement. Rats completed training when they reached criterion performance (d' $\geq 1$; see below) for 3 consecutive sessions (Fig. 24C).

Naïve group of rats $(\mathrm{n}=4)$ was not trained. These animals were under food restriction, passed a handling phase (Stage 1) and an anti-neophobia period (Stage 2), but for the rest of steps (stages 3, 4 and 5) they were only taken into the operational cage for 30 min with 5 initial pellets and other final 5 pellets, without applying any paradigm.

\section{Behavioral protocols}

We generated different behavioral protocols to confirm the animal's ability to discriminate and detect pure tones.

Oddball sequence task ( 3 sessions): Animals need to detect a low probability deviant tone $(10 \%)$ in a sequence of high probability $(90 \%)$ regular tones (standard tones). Frequency contrast between tones was set at 0.5 octaves (Fig. $25 \mathrm{~A})$. Each pure tone ( $70 \mathrm{~dB}$, duration $200 \mathrm{~ms}$, risefall $10 \mathrm{~ms})$ were spaced by an interstimulus interval of $1.5 \mathrm{~s}$ (onset to onset; ISI) and with a response window of 
$1.49 \mathrm{~s}$. Frequencies used for each group, as it has been described before, are specified in the table 1. Maximum number of standard (1259) and deviant (141) tones were calculated for a session of $30 \mathrm{~min}$ (1400 tones). First 5 stimuli of each session were standards, and each deviant tone was always preceded by a minimum of 3 standards tones. We acquired data of 3 consecutive sessions applying this sequence to every animal (including naïve ones). Responses were quantified using d' values.

Deviant generalization task ( 3 sessions): As in the previous task, animals need to detect a low probability deviant tone $(10 \%)$ in a sequence of high probability $(90 \%)$ standard tones, but frequency contrast was modified on a daily basis and increased in 0.25 octaves steps for 3 consecutive sessions. Standard frequency was fixed across sessions, and the frequency of the deviant tone was $0.75,1.0$ and 1.25 octaves larger than the standard frequency in sessions 1,2 , and 3 , respectively (Fig. 25B). Responses were quantified using d'values.

Many-deviants task ( 3 sessions): Animals need to detect any deviant tone (10\%) presented in a sequence of a constant high probability (90\%) standard tone. Each deviant tone has a frequency randomly selected between 9 possibilities $(4.0,4.8$, $5.7,6.7,8.0,9.5,11.3,13.5,16.0$ and $19.0 \mathrm{kHz}$ ), excluding the standard frequency for each group. This result is a $1.11 \%$ probability of occurrence for each different deviant. The standard tone frequency remained unchanged during the task (4.8,5.7, 6.7 or $8.0 \mathrm{kHz}$; Fig. 25C and table 2). Discrimination index (dprime) was quantified for all deviant tones together, regardless the frequency 
presented. Once finished the last session, animals had food ad libitum for 3 days and a final weigh was measured.

\section{DATA AND STATISTICAL ANALISYS}

Data and statistical analysis of deviance detection in physiologically identified cell types in the rat auditory cortex (SECTION I)

Once the data (spike times and waveforms) was collected from electrophysiological recordings, the first step of offline processing was spike sorting, which allowed removal of recorded artefacts and noise, and occasionally separate single units. Spike sorting was performed on OpenSorter (TuckerDavis $\AA^{\circledR}$ Technologies), using a supervised Bayesian procedure. Spike waveforms were aligned on the trough. The mean waveform of the spikes of each unit was calculated, and the following measurements were made on the average waveform: time and voltage of the peak and trough, spike amplitude (peak-totrough) and spike half-width (spike width at $50 \%$ of the peak-to-trough amplitude; hereinafter "spike width") (Fig. 12A). We used the peak-to-trough signal to noise ratio (SNR) as an indicator of the quality of the spikes. The SNR was calculated as the difference between the maximum and minimum voltages, divided by the standard deviation of the background noise (when there were no spikes). Only units with an SNR $>5$ were included in the study (Fig. 12B). The average number of spikes per sorted unit was $3207 \pm 4765$ spikes (range: $21-35610$ spikes) (Fig. $12 \mathrm{C}$ ); about $95 \%$ of the units included more than 200 spikes. Inter-spike intervals were measured over the oddball sequences for each unit, and were expressed 
as its reciprocal, the instantaneous firing rate (Kostal et al., 2018). We then calculated the distribution of instantaneous firing rates for each unit, and took as reference for the neuronal discharge the values of the $50^{\text {th }}, 25^{\text {th }}, 10^{\text {th }}, 5^{\text {th }}$ and $1^{\text {st }}$ percentiles, in order to account for the average as well as for the fastest firing rates that each neuron could achieve, respectively.

All statistical analyses were performed in MATLAB. Since most value distributions did not hold the normality assumption (both by visual inspection and by the results of the Lilliefors normality test), non-parametric tests were employed in this study. We used the Wilcoxon rank-sum test to check whether two independent samples came from distributions with equal medians. The sign test was used to determine whether the median of a group was different from zero. When performing comparisons between two groups (or comparing one group to zero) multiple times (Fig. 16), the resulting p-values were corrected using the Holm-Bonferroni method. For comparisons of distributions between more than two groups (e.g., all the comparisons between cortical fields), the Kruskal-Wallis test was used; post hoc comparisons to find differences between groups were made using the Bonferroni method. The average values of the distributions are expressed as mean \pm SD unless indicated otherwise. Null hypotheses were rejected at a significance level of 0.05 .

Data and statistical analysis of cholinergic modulation of SSA and prediction error responses (SECTION II) 
The degree of SSA was quantified by the CSI, reported previously (Malmierca et al., 2009; Ulanovsky et al., 2005, 2003) . The CSI reflects the difference between the neural responses to the deviant and standard stimuli, normalized to the total of responses to both stimuli, and is defined as:

$$
C S I=\frac{d\left(f_{1}\right)+d\left(f_{2}\right)-s\left(f_{1}\right)-s\left(f_{2}\right)}{d\left(f_{1}\right)+d\left(f_{2}\right)+s\left(f_{1}\right)+s\left(f_{2}\right)}
$$

where $d\left(f_{i}\right)$ and $s\left(f_{i}\right)$ are responses to each frequency $f_{1}$ or $f_{2}$ when they were the deviant $(d)$ or standard $(s)$ stimulus in the oddball paradigm, respectively. For the subset of experiments where we recorded the many-standards and cascade controls, we compared the responses to the same physical stimulus when it took the role of a standard, a deviant (ascending or descending, depending on whether the preceding standard was of lower or higher frequency, respectively), or was part of a cascade sequence (matching the ascending or descending condition of the corresponding deviant). Alongside the oddball paradigm, we recorded responses of neurons to two cascade sequences (ascending and descending), which consisted of 10 tones selected within the FRA presented in a predictable succession of increasing or decreasing frequencies. We did not include the many-standards control in these analyses because these experiments are time consuming and the need of holding the recording neuron for long enough before, during and after the drug injection. However, we have previously demonstrated that the many-standards and cascade controls responses were comparable, and the latter is considered to be more rigorous. (Casado-Román et al., 2019; Parras et al., 2017; Ruhnau et al., 2012; ValdésBaizabal et al., 2020).

The responses of each neuron were normalized as

Normalized Deviant $=$ Deviant $/ N$ 


$$
\begin{gathered}
\text { Normalized Standard }=\text { Standard } / N \\
\text { Normalized Control }=\text { Control } / N
\end{gathered}
$$

where

$$
N=\sqrt{\text { Deviant }^{2}+\text { Standard }^{2}+\text { Control }^{2}}
$$

is the Euclidean norm of the vector defined by the deviant, standard and cascade responses, so that the normalized responses take values in the range $0-1$.

With these normalized baseline-corrected spike counts, we next computed the indices of neuronal mismatch (iMM), repetition suppression (iRS), and prediction error (iPE) as:

$$
\begin{gathered}
i M M=\text { Normalized Deviant }- \text { Normalized Standard } \\
i R S=\text { Normalized Control }- \text { Normalized Standard } \\
i P E=\text { Normalized Deviant }- \text { Normalized Control }
\end{gathered}
$$

Index values ranged between -1 and 1 and facilitated the quantitative decomposition of neuronal mismatch into RS and PE since

$$
i M M=i R S+i P E
$$

Which is largely comparable to the CSI calculated for the oddball paradigm (Parras et al., 2017).

In order to determine a significant effect of the drugs on the CSI, we calculated an empirical distribution of CSI values by performing 2000 bootstraps of the responses to each standard or deviant stimulus for each neuron in the control condition, from which we determined a 95\% confidence interval. An effect was considered significant at $\alpha=0.05$ if the CSI during the drug condition fell beyond the limits of the control CSI confidence interval. All results were analyzed using a paired t-test comparing control condition versus drug application; all data are 
reported as mean $\pm S D$. If the data were found to be non-normally distributed, a non-parametric Mann-Whitney (for independent data) or Wilcoxon Signed Rank test (for paired data) was performed. All of the data analyses were performed with Sigma Plot $\AA$ v12.5 and MATLAB software, using the built-in functions, the Statistics and Machine Learning toolbox, or custom scripts and functions developed in our laboratory.

Data and statistical analysis of effect of behaviorally relevant sounds in deviant detection and prediction error responses (SECTION III)

To determine the animal's ability to recognize deviant tones intermingled in the paradigms presented, we calculated the so-called d' discrimination index (adapted from Green \& Swets, 1966; 1988): $d^{\wedge \prime}=f^{\prime \prime} 1 "(x, \mu, \sigma)-f^{\prime \prime} 2 "(y, \mu, \sigma)$ Where $f$ is the normal distribution of the probability of $x(\mathrm{HIT})$ or $y(\mathrm{FA}), \mu$ is the mean of the distribution and $\sigma$ is the standard deviation. In the cases of sessions with minimum (0) or maximum (141) of hits or FA, it was not possible to calculate d', and application of Hautus' correction was necessary. It consisted in adding 0.5 to both the number of hits and the number of false alarms, and add 1 to both the number of signal trials and the number of noise trials; dubbed the loglinear approach (Hautus, 1995). All statistical comparisons were made using Sigmaplot 12.5 (Systat Software, Icn., Germany), including ANOVA tests for repeated measures, Holm-Sidak method for multiple comparisons and paired t-test comparing trained and untrained conditions. 


\section{RESULTS}

The results obtained in this thesis work will be presented in three separate sections. The first section describes the neuronal basis of the circuitry responsible for deviance detection. The second section shows the modulation of acetylcholine on SSA and prediction error in the rat auditory cortex. Finally, the third section shows how the training of rats through acoustic behavioural paradigms generates increases the prediction error responses in the A1.

While I have performed all the experiments and analysed all the data related to section 2 on the effects of ACh on deviance detection, the first and third sections are the result of two fruitful collaborations with other members of our laboratory CANElab (Section 1: Pérez-González et al., 2020; Section 3: Morado-Díaz et al., 2020). 


\section{Section I: deviance detection in physiologically identified cell types in the rat auditory cortex}

The results of this study are published in Pérez-González et al. (2020). We recorded 282 neurons from the $\mathrm{AC}$ of anaesthetized rats and classified them as putative excitatory or inhibitory units. We aimed to test whether they have different deviance detection properties as it is generally accepted that the fast spiking (FS) and regular spiking (RS) neurons are putative inhibitory and excitatory, respectively (Mountcastle et al., 1969). For this, we characterized cortical neurons based on the shape of their spike waveforms and their firing rates. This study analyses the spike waveforms from a data set of 282 neuronal recordings, originally collected for other studies (Nieto-Diego et al., 2016; Parras et al., 2017) regarding neuronal adaptation and predictive coding in the AC from a total of of 86 urethane-anaesthetized rats. In this study, we found that the deviance detection occurs in both excitatory and inhibitory neurons.

For the analysis of these results, we performed a spike sorting analysis (performed on OpenSorter(TDT)), which allowed removal of recorded artifacts and noise. The mean waveform of the spikes of each unit was calculated, and the following measurements were made on the average waveform: time and voltage of the peak and trough, spike amplitude (peak-to-trough) and spike halfwidth (spike width at $50 \%$ of the peak-to-trough amplitude; hereinafter "spike width"; Fig.12A). We used the peak-to-trough signal to noise ratio (SNR) as an indicator of the quality of the spikes. The SNR was calculated as the difference between the maximum and minimum voltages, divided by the standard deviation 
of the background noise (when there were no spikes). Only units with an SNR $>5$ were included in the study (Fig. 12B). The average number of spikes per sorted unit was $3207 \pm 4765$ spikes (Fig. 12C); about $95 \%$ of the units included more than 200 spikes.
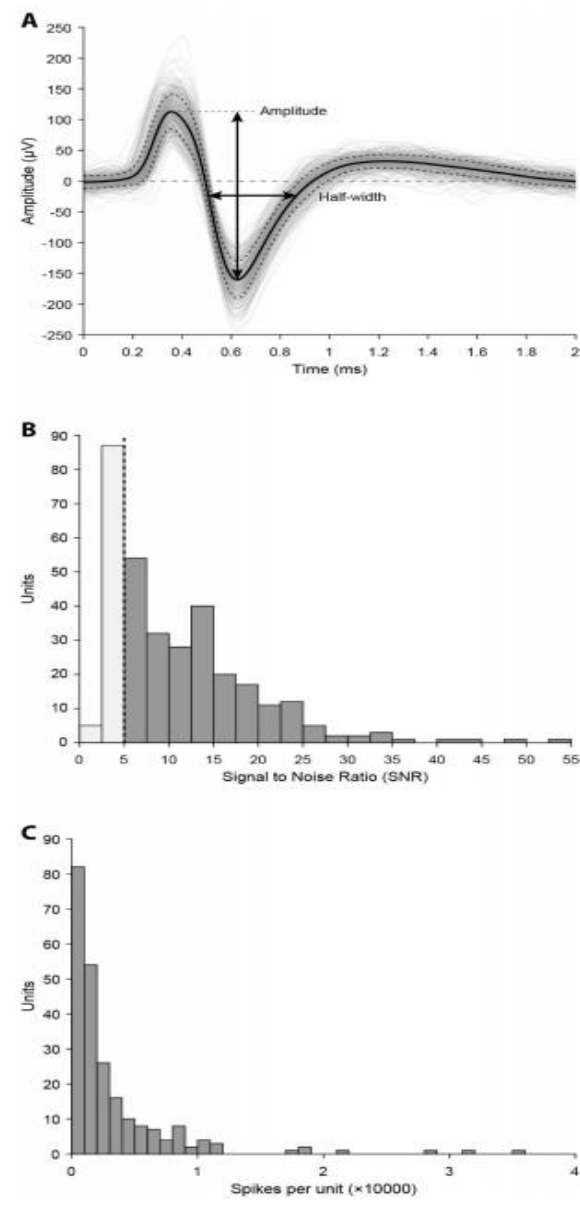

Fig 12. Spike width measurement. (A) The spikes for each isolated unit were aligned on the trough. The mean (black solid line) and standard deviation (dotted black lines) of the spikes of each unit was calculated. Based on the mean waveform, we measured the peak-totrough amplitude (vertical line) and spike half-width (spike width at $50 \%$ of the peak-to-trough amplitude). (B) Distribution of the peakto-trough signal to noise ratio (SNR). Only units with an $\mathrm{SNR}>5$ were included in the study (dotted line). (C) Distribution of the number of spikes per unit. About 95 $\%$ of the units included more than 200 spikes.

Regular and fast spiking of auditory cortical neurons

Using spike sorting, we were able to collect 323 well isolated single units (241, 37 and 4 sites yielded 1, 2 and 3 units per site, respectively) and measure their spike waveforms. Fig.13A shows the distribution of spike widths in our sample. These units were classified as regular spiking or fast spiking depending on the shape of the spike width distribution, which has a bimodal appearance with a prominent peak towards narrow spikewidths and a spread plateau towards wider spike widths. According to this distribution, we set a cut-off at $0.35 \mathrm{~ms}$; units with 
spike widths larger than 0.35 ms were classified as RS (Fig. 13A, orange), and the rest, i.e. units with spike widths shorter than $0.35 \mathrm{~ms}$, as FS (Fig. 2A, blue). In our sample, 60 (26\%) units were classified as RS, and the rest as FS (171, 74\%)(Fig. 13B).
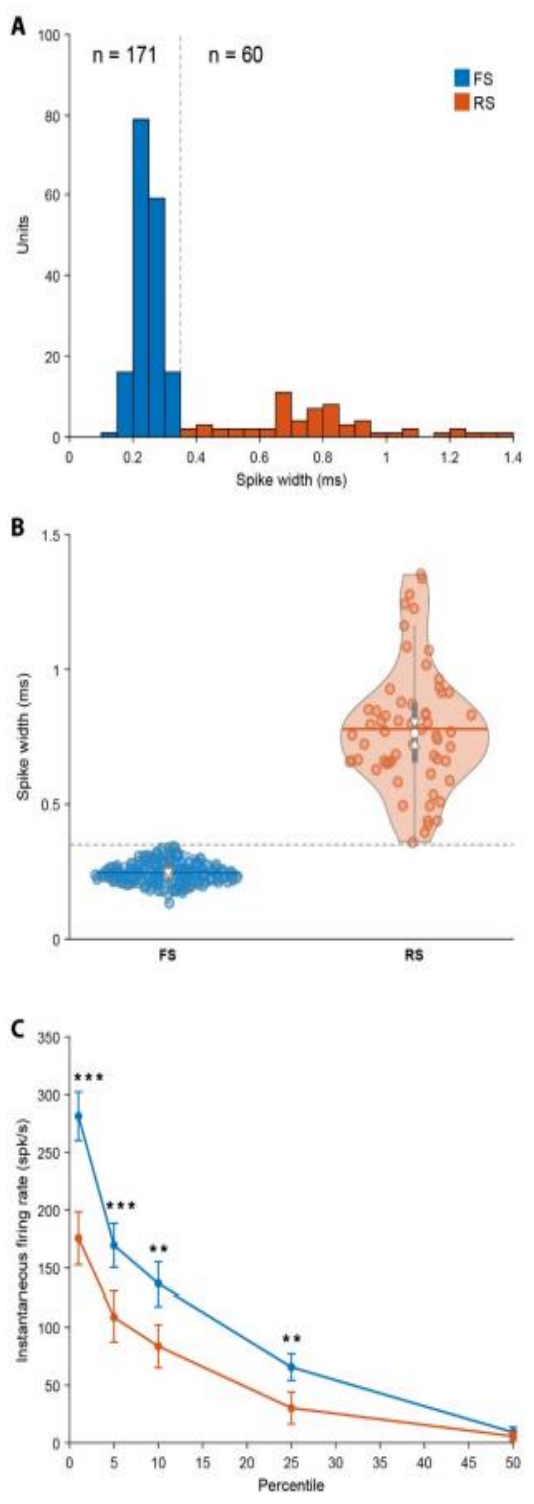

Fig. 13. Classification of units based on spike width and firing rate. (A) Distribution of spike widths measured in our sample. Units with a spike width larger than $0.35 \mathrm{~ms}$ (dashed grey line) were classified as regular spiking (RS, orange), while the rest were classified as fast spiking (FS, blue). (B) Violin plot showing the distribution of spike widths in RS and FS units. In this and similar violin plots, the horizontal solid line indicates the mean of the distribution, while the white circle indicates the median. The thick grey bar expands from the first (Q1) to the third quartile (Q3), and the whiskers show the range of lower and higher adjacent values (i.e. values within 1.5 IQR below Q1 or above Q3, respectively). The triangles indicate a $95 \%$ confidence interval for the median. (C) The instantaneous firing rate (median $\pm 95 \%$ confidence interval) was significantly lower for RS than for FS units for the 25th, 10th $\left({ }^{* *} p<0.01\right), \quad 5$ th and 1 st percentiles $\left({ }^{* * *} p<0.001\right)$, but not for the 50th percentile.

Spike amplitude, cortical field and cortical layer localization

In this work, the neuronal responses were recorded during auditory stimulation using an oddball paradigm. We measured the firing rates in the periods between stimuli (during the period of $50 \mathrm{~ms}$ before each stimulus), in order to estimate the 
spontaneous firing rates, but there were no differences between RS and FS units ( $p=0.129$, Wilcoxon rank-sum test). To look for differences between RS and FS units, we measured the peak-to-trough amplitude (Fig. 14C-D) of the average spike waveforms. We found that RS units had larger amplitudes than FS spikes. The peak-to-trough amplitude of RS units $(571.76 \pm 510.59 \mathrm{mV})$ was much larger than the amplitude of FS units $(147.61 \pm 93.55 \mathrm{mV} ; p<0.001$, Wilcoxon rank-sum test) (Fig. 14C). The spike amplitudes were similar among AC fields for RS units (Kruskal-Wallis, $\mathrm{X} 2(4, N=54)=3.27, \mathrm{p}=0.514)$ and FS units(Kruskal-Wallis, $\mathrm{X} 2(4$, $N=151)=1.68, \mathrm{p}=0.514)$. On the other hand, based on stereotaxic coordinates and response characteristics, we assigned 205 units to any of the five main fields of the rat $\mathrm{AC}$ (Fig. 14A). Thus, 111 units were located in lemniscal fields $(\mathrm{A} 1=$ $66 ; \mathrm{VAF}=33 ; \mathrm{AAF}=12)$ and 94 in non-lemniscal fields $(\mathrm{SRAF}=47 ; \mathrm{PAF}=47)$. Lemniscal fields contained a larger proportion of FS units ( $81 \%$ across all lemniscal fields; $A 1,85 \%$; VAF,70\%, AAF, 92\%) than non-lemniscal fields (65\% across all non-lemniscal fields; SRAF, 64\%; PAF, 66\%). When comparing the spike widths of both types of units across AC fields (Fig. 14B), we found no differences for RS units (Kruskal-Wallis, $\left.\chi^{2}(4, N=54)=4.04, p=0.401\right)$ nor for FS units (Kruskal-Wallis, $X 2(4, N=151)=8.54, p=0.074)$ (Fig. 14B). The peakto-trough amplitude of RS units $(571.76 \pm 510.59 \mathrm{mV})$ was much larger than the amplitude of FS units $(147.61 \pm 93.55 \mathrm{mV} ; \mathrm{p}<0.001$, Wilcoxon rank-sum test)(Fig. 14C). The spike amplitudes were similar among AC fields for RSunits (Kruskal-Wallis, $\mathrm{X} 2(4, N=54)=3.27, \mathrm{p}=0.514)$ and FS units (Kruskal-Wallis, $\mathrm{X} 2$ $(4, N=151)=1.68, \mathrm{p}=0.514)$. 

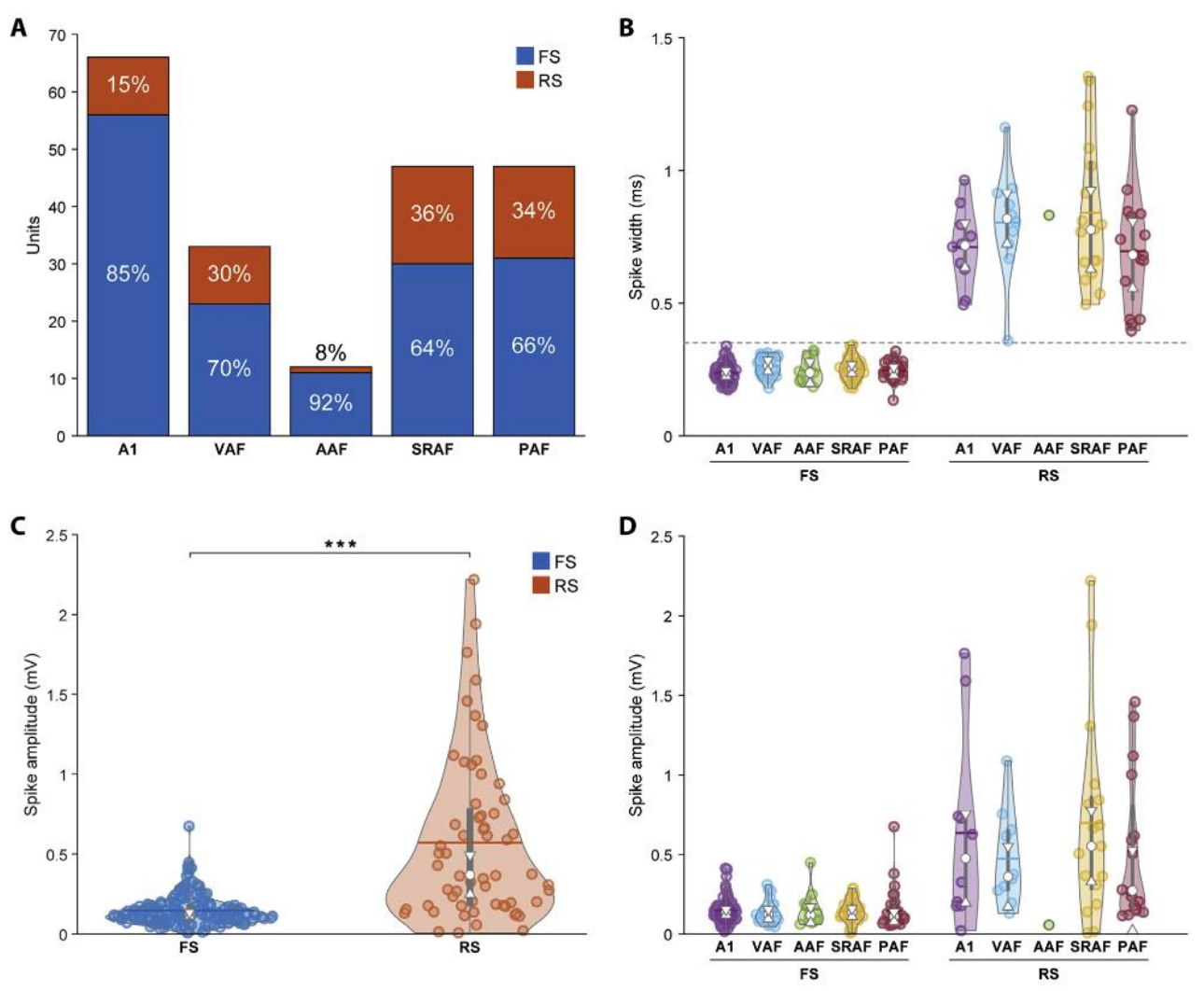

Fig. 14. Location of RS and FS units in AC cortical fields and spike amplitude. (A) Number and percentage of RS (orange) and FS (blue) units located in the A1, VAF, AAF, SRAF and PAF. (B) Distribution of spike widths in RS and FS units for each auditory field. There were no significant differences in the distribution of spike widths across fields for neither RS nor FS units. (C) Distribution spike amplitudes for RS and FS units. The amplitude of RS units was significantly larger than that of FS units $\left({ }^{* * *} p<0.001\right)$. (D) Distribution of spike amplitudes in RS and FS units for each auditory field. There were no significant differences in the distribution of spike amplitudes across fields for neither RS nor FS units.

Next, in order to check whether RS and FS units had any preferential location across the cortical layers, we recorded the depth of the electrode tip from the brain surface for 202 units. The most superficially located unit was recorded at $115 \mathrm{~mm}$, while the deepest unit was found at $1170 \mathrm{~mm}$ (mean: $584 \pm 247 \mathrm{~mm}$ ). RS and FS unitswere found at similar depths $(596 \pm 238$ vs. $579 \pm 251 \mathrm{~mm}$, respectively; $p=0.800$, Wilcoxon rank-sum test; Fig. $15 \mathrm{~A}$ ). There were no significant differences between AC fields in the depth at which RS units (Kruskal- 
Wallis, $\left.X^{2}(4, N=54)=9.30, p=0.054\right)$ nor FS units (Kruskal-Wallis, $X^{2}(4, N=$ 148) $=5.20, p=0.267)$ were recorded (Fig. 15B-C).

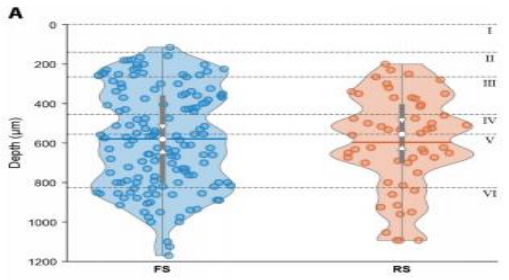

B
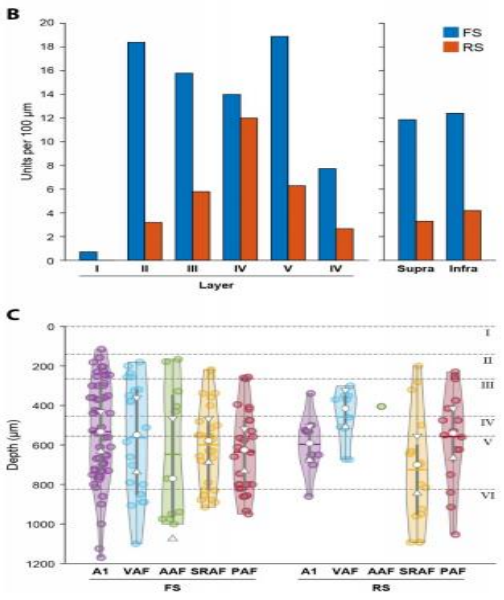

Fig. 15. Location of RS and FS units in cortical layers. (A) Depth of the recording sites for RS and FS units. (B) Left side: density of RS and FS units per layer, calculated as number of units per $100 \mathrm{~mm}(\mathrm{n}=$ 202). RS units were most commonly found in layers IV and V, while FS units spread over layers II and V. Right side: density of RS and FS units in supragranular (I-III) and infragranular (V-IV) layers. (C) Distribution of recording site depths of RS and FS units for each auditory field. There were no significant differences in the distribution of spike amplitudes across fields for FS units nor for RS units.

The prediction error and repetition supression indices

Finally, we analyzed the relationship between the FS and RS units with the prediction error and repetition supression indices, obtained through the MSCCascade paradigms (Parras et al., 2017) (Fig. 11). The index of repetition suppression is a measurement of neuronal adaptation to standard stimuli compared to a cascade control sequence; the average for all units was $0.162 \pm$ 0.220. The medians for both RS and FS units were larger than zero (both $p<0.001$, sign test), and there were no statistical differences in the index of repetition suppression between both groups $(0.178 \pm 0.300$ vs $0.157 \pm$ $0.183 ; p=0.632$ Wilcoxon rank-sum test) (Fig. 16C). Similarly, there were no differences between RS and FS units when looking at each individual AC field. 
The median index of repetition suppression was larger than zero in all AC fields except AAF for FS units (A1, $p<0.001$; VAF, $p<0.001$; AAF, $p=0.262$; SRAF, $p<0.001$; PAF, $p<0.020$ ), but only in SRAF for RS units $(A 1, p=1.000$; VAF, $p=0.328 ; A A F, p=1.000 ;$ SRAF, $p=0.016 ; P A F, p=0.106$ ). There were differences in the index of repetition suppression among AC fields for RS units (KruskalWallis, $x 2(4, N=54)=9.53, p=0.049)$ but not for FS units (Kruskal-Wallis, $x 2$ $(4, N=151)=7.46, p=0.114)$ (Fig. 16D). However, post hoc comparisons did not find any differences between groups for RS units, although the comparison between A1 and SRAF was close to the level of significance $(p=0.057)$. The index of prediction error indicates how much does a neuron respond to deviant stimuli as compared to a cascade control sequence the average for all units was $0.403 \pm$ 0.357. The medians for both RS and FS units were larger than zero (both $p<0.001$, sign test), and there were no statistical differences between both groups ( $0.335 \pm 0.431$ vs $0.427 \pm 0.324 ; p=0.310$, Wilcoxon rank-sum test) (Fig. $16 \mathrm{E})$. Similarly, there were no differences between RS and FS units when looking at each individual AC field. The median index of prediction error was larger than zero in all $A C$ fields except VAF for FS units $(A 1, p<0.001$; VAF, $p=0.173$; AAF, $p=0.007$; SRAF, $p<0.001$; PAF, $p=0.002$ ), and in no fields for RS units ( $A 1$, $p=0.328 ; \mathrm{VAF}, \mathrm{p}=0.219 ; \mathrm{AAF}, \mathrm{p}=1.000 ; \mathrm{SRAF}, \mathrm{p}=0.196$; $\mathrm{PAF}, \mathrm{p}=0.128)$. The index of prediction error was similar in all the AC fields, for both RS units (KruskalWallis, $\left.\quad x^{2} \quad(4, N=54)=5.73, p=0.220\right)$ and FS units (Kruskal-Wallis, $\quad x^{2}$ $(4, N=151)=1.89, p=0.755$, Fig. $16 F)$. 

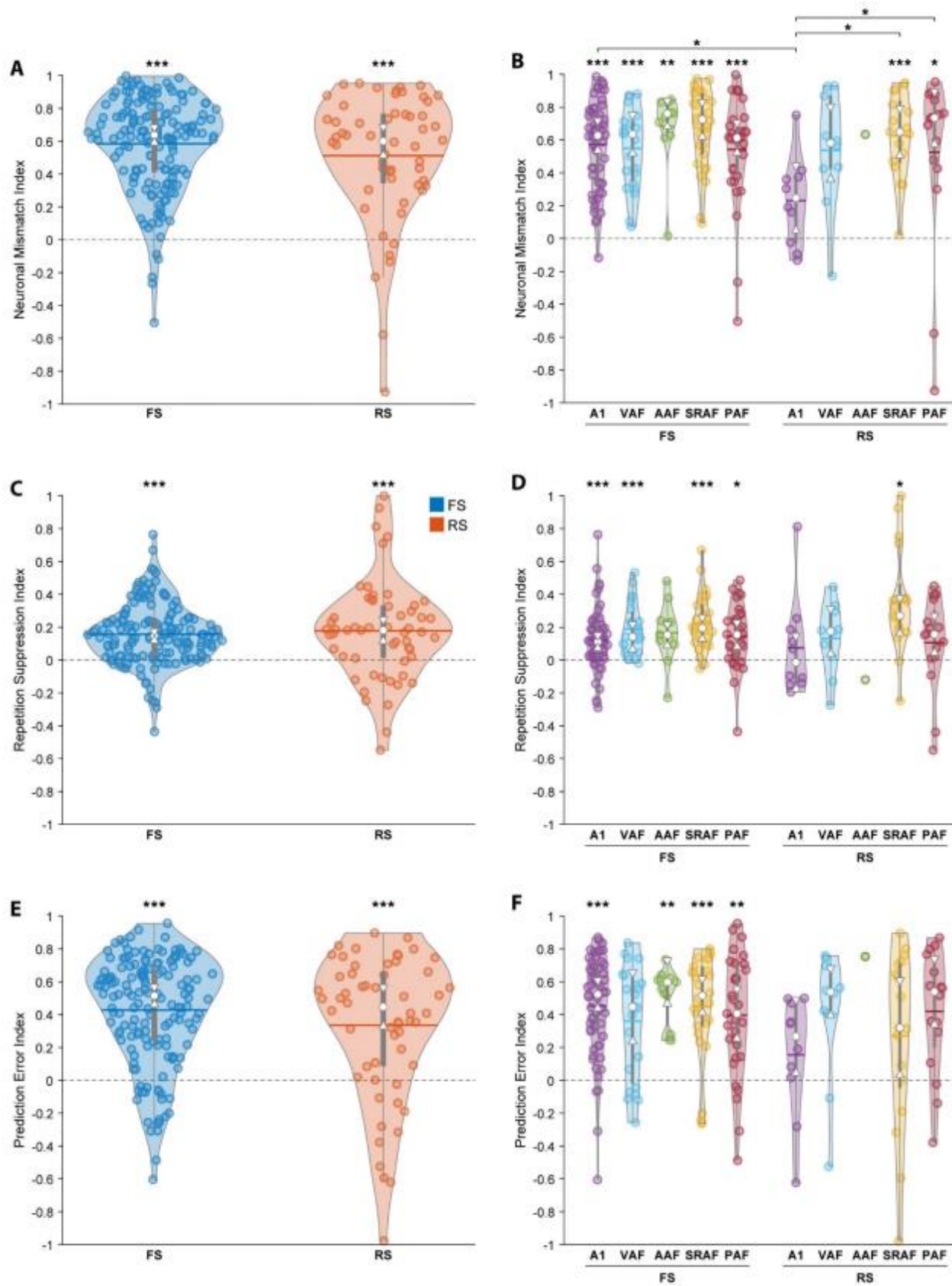

Fig. 16. Indexes of Neuronal Mismatch, Repetition Suppression and Prediction Error. (A-B) Distribution of the index of neuronal mismatch of RS and FS units for each auditory field. (C-D) Distribution of the index of repetition suppressionof RS and FS units for each auditory field.(E-F) Distribution of the index of prediction error of RS and FS units for each auditory field. Asterisks above the violin plots indicate that the group average is different from zero; brackets indicate differences between groups $\left({ }^{*} p<0.05,{ }^{* *} p<0.01,{ }^{* * *} p<0.001\right)$. 
In summary, thus far the principal conclusions of this work include: 1) measurements of spike widths in extracellular recordings from the anesthetized rat AC can be used to classify the units as putative excitatory or inhibitory; 2) both putative excitatory and inhibitory neurons in the rat $A C$ show similar levels of deviance detection; 3) both types of neurons reflect a predominance of the prediction error signaling rather than repetition suppression and 4) the involvement of both types of neurons in the circuits contribute to generate deviance detection. 


\section{Section II: Acetylcholine modulates SSA and prediction error response in auditory rat cortex}

In this section, I describe how cholinergic modulation affects SSA and prediction error after using the oddball paradigm and other appropriate control stimulation paradigms. For this, we recorded a total of 114 units in the AC before, during and after the microiontophoretic injection of acetylcholine $(n=99)$. We also applied two antagonist drugs: scopolamine $(n=13)$ and mecamylamine $(n=10)$ to test if the effects observed with ACh were mediated by muscarinic or nicotinic receptors, respectively. Recording depths ranged 140-1080 $\mu \mathrm{m}$ including neurons from all layers, except layer I.

In order to allocate each recorded neuron to a specific field in the AC, we recorded the FRA and analyzed the topographical distribution of CF for each unit. Each recording was assigned to a dorsoventral and rostrocaudal coordinate system relative to bregma as in previous studies (Nieto-Diego and Malmierca, 2016; Parras et al., 2017b; Polley et al., 2007). This analysis allowed us to pool the data from all animals (Fig. 17A) and construct a synthetic map of the CF across the entire rat auditory cortex (Nieto-Diego and Malmierca, 2016; Parras et al., 2017). We determined a high-frequency reversal zone between ventral auditory field (VAF, caudally) and anterior auditory field (AAF, rostrally), a low-frequency reversal zone between $\mathrm{A} 1$ and posterior auditory field (PAF, dorsocaudally), and a high-frequency reversal between VAF and suprarhinal auditory field (SRAF, ventrally). Thus, we could reliably define the lemniscal (A1, AAF, and VAF) and non-lemniscal (SRAF, PAF) auditory cortical fields as shown in Fig. 17B. 

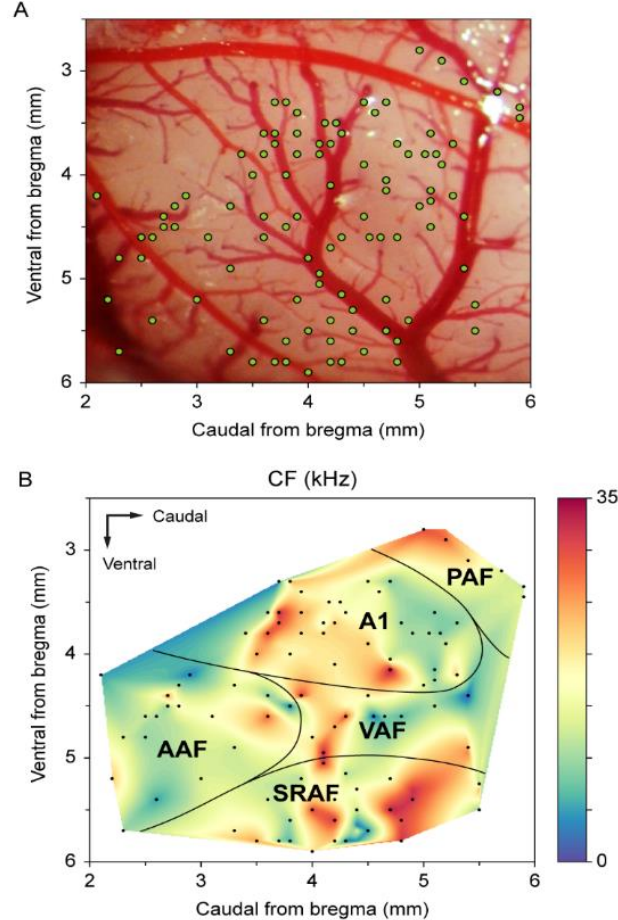

Figure 17: Map of all recording locations. (A) All recording sites drawn over the cortex of a representative animal. At every site, the CF was determined and then we presented an oddball paradigm and the corresponding control sequences.

Distribution of the CFs across the entire rat auditory cortex. Note how each field shows a characteristic CF gradient.

Figure 18 illustrates representative FRAs of five units from each of the auditory fields before, during and after ACh microiontophoretic injection. As in these example cells, we observed an increase in the mean of firing rate values after the ACh injections (mean control firing rate: $0.80 \pm 0.49$; mean ACh firing rate: $1,61 \pm 0,91$. Wilcoxon Signed Rank test, $p<0.001)$. Most neurons recovered their basal firing rates after 60-90 minutes post ACh injection (recovery firing rate mean: $0.84 \pm 0.42)$. After the FRA was measured, we selected a pair of pure tones (10-30 dB above minimum threshold) within the FRA at each recording site to test the adaptation. In all the multiunits, the FRA response was recorded, which helped to identify the cortical field (lemniscal or non-lemniscal). Figure 18 illustrates representative FRAs of five units from each of the auditory fields before, during and after ACh microiontophoretic injection. As in these example cells, we observed an increase in the mean of firing rate values after the ACh 
injections (mean control firing rate: $0.80 \pm 0.49$; mean ACh firing rate: $1.61 \pm 0.91$. Wilcoxon Signed Rank test, $p<0.001)$. Most neurons recovered their basal firing rates after 60-90 minutes post ACh injection (recovery firing rate mean: $0.84 \pm$ $0.42)$. After the FRA was measured, we selected a pair of pure tones (10-30 dB above minimum threshold) within the FRA at each recording site to test the adaptation properties of the units ( $f 1$ and $f 2$ in Fig. 18).



Figure 18: Examples of FRA changes in control, ACh and recovery conditions: Each row shows the FRA for a representative unit of each $A C$ field (lemniscal: A1, AAF and VAF; and non-lemniscal: SRAF and PAF). In all the examples, the spike count increased after ACh injection ("Acetylcholine" column) and most of these neurons recovered their basal firing rates after 6090 minutes post ACh injection ("Recovery" column). The "AcetylcholineControl" column shows the difference in firing rate between ACh and control conditions. f1 and f2 indicate the location of the frequencies used for the oddball sequence on each unit. 
Electrophysiological responses in AC using oddball paradigms

To analyze the effect of ACh on SSA, we recorded the neuronal responses under an auditory oddball paradigm and computed the CSI to quantify the SSA levels in the rat auditory cortex. Dot raster and PSTH responses were obtained in all neuronal responses, with and without ACh. Figure 19 shows five examples of units from each auditory cortical field recorded (PAF, A1, VAF, AAF and SRAF) before, during and after the injection of ACh (Fig. 19). An increase in SSA levels is observed in all individual examples, mainly due to an increase in firing rate in response to the deviant tone. 

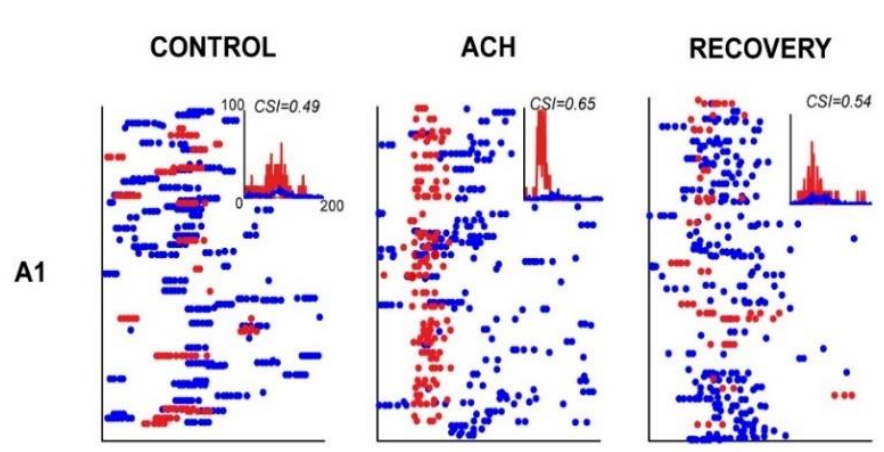

Figure 19: Examples of neuronal responses in control, $\mathrm{ACh}$ and recovery conditions. The figure shows dot rasters and peri-stimulus time histograms (PSTH, insets) of five representative units (rows) in control (left
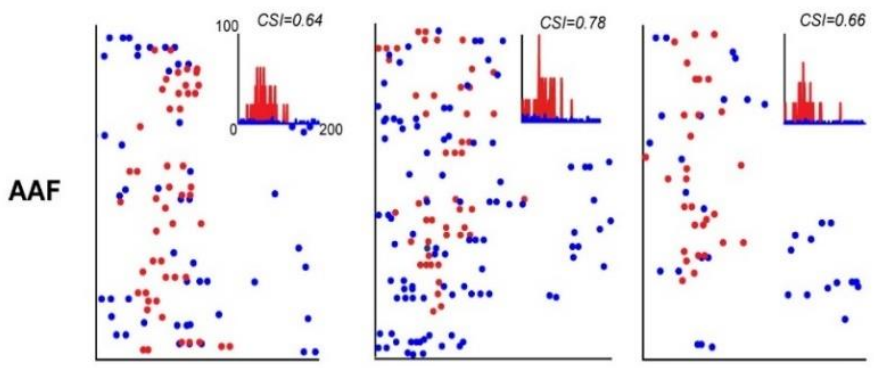

column), ACh (central column) and recovery conditions (right column) from lemniscal (A1, AAF and VAF) and nonlemniscal (SRAF and PAF) fields. In all the examples,
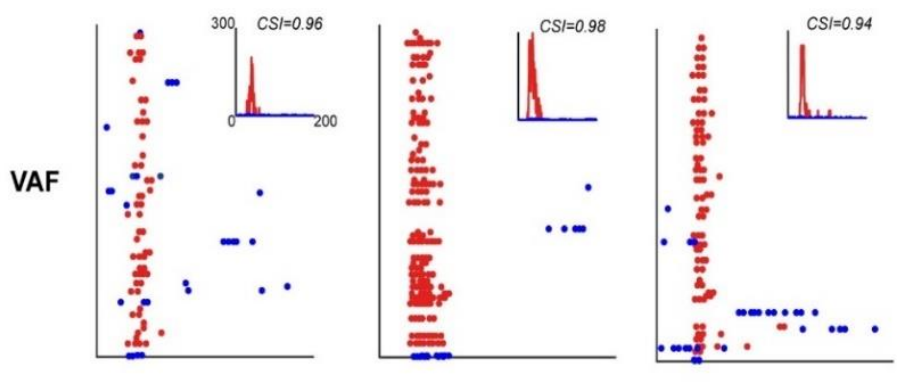
the SSA levels (CSI) increased during ACh injection, mainly due to an increase in the firing rate in response to the deviant tone (red), rather than changes in the response to
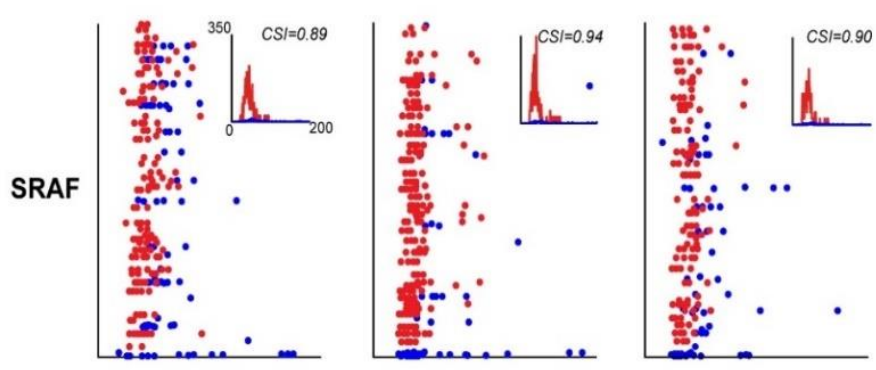
standard tones (blue).
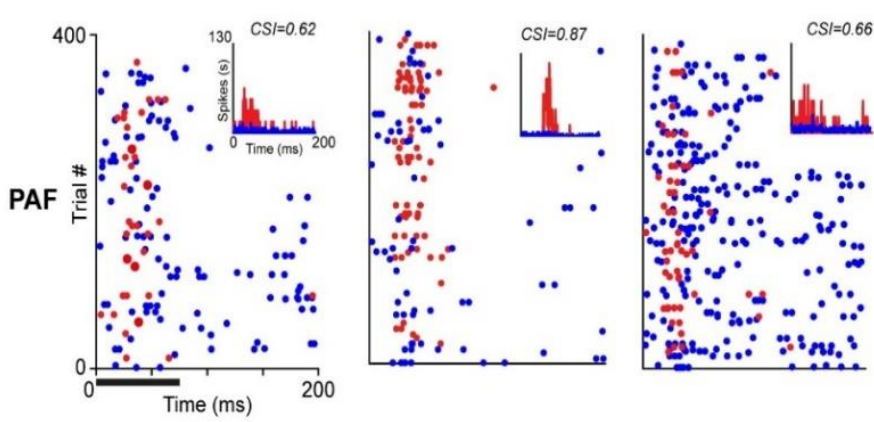

Our sample $(n=99)$ covers a wide range of CSI values, from 0.02 to 0.93 , greatly enhancing our power to study the action of ACh. A remarkable finding was that ACh produced larger CSI increases to neurons with low SSAs, ranging from 0.02 
to 0.6 (CSI control: $0.37 \pm 0.16$ vs drug: $0.54 \pm 0.19 ; 71$ of 99 units). By contrast, neurons with CSI values larger than 0.6 showed smaller increases in SSA after ACh (CSI control: $0.76 \pm 0.10$ vs drug: $0.84 \pm 0.09$; 28 of 99 units). This may reflect a ceiling effect, as neurons with low levels of SSA have a larger range for SSA to increase. We found that the difference between control and ACh CSI levels for all units followed a linear distribution $\left(R^{2}=0.17\right)$ and further support that ACh injection exerted larger changes on neurons with SSA levels below 0.6 (Fig. 20A). Afterwards, we analyzed the effect of ACh on the response to the deviant or standard stimuli separately and observed that ACh produced a significant increase in the spike count in response to the deviant tones (Fig. 20B; $1.62 \pm 1.12$ spikes/stimulus), as compared to the control condition $(0.80 \pm 0.69$ spikes/stimulus; Wilcoxon Signed Rank test, $p<0.001$ ) while the magnitude of the response to standard tones remained unchanged (Fig. 20B; $0.40 \pm 0.49$ vs $0.41 \pm 0.42$ spikes/stimulus, control vs ACh; Wilcoxon Signed Rank test, $p=$ 0.08). These results clearly show that ACh has a differential effect in $A C$ neurons, increasing the responses to rare and unexpected stimuli but not to common and expected stimuli. The average CSI during ACh injection $(C S I=0.62 \pm 0.22)$ was significantly larger than that of the control condition (CSI $=0.48 \pm 0.23$; Wilcoxon Signed Rank test, $p<0.001)$. Most of these neurons recovered their baseline CSI levels when the drug effects ended (CSI $=0.44 \pm 0.24$; Fig. 20C). The effect of ACh on CSI was consistently observed on each individual AC field (Fig. 20D); although CSI values with ACh injection were lower in lemniscal (A1: 36 units, AAF: 20 units and VAF: 12 units) compared to non-lemniscal fields (PAF: 6 units and SRAF: 25 units) during the control condition. 

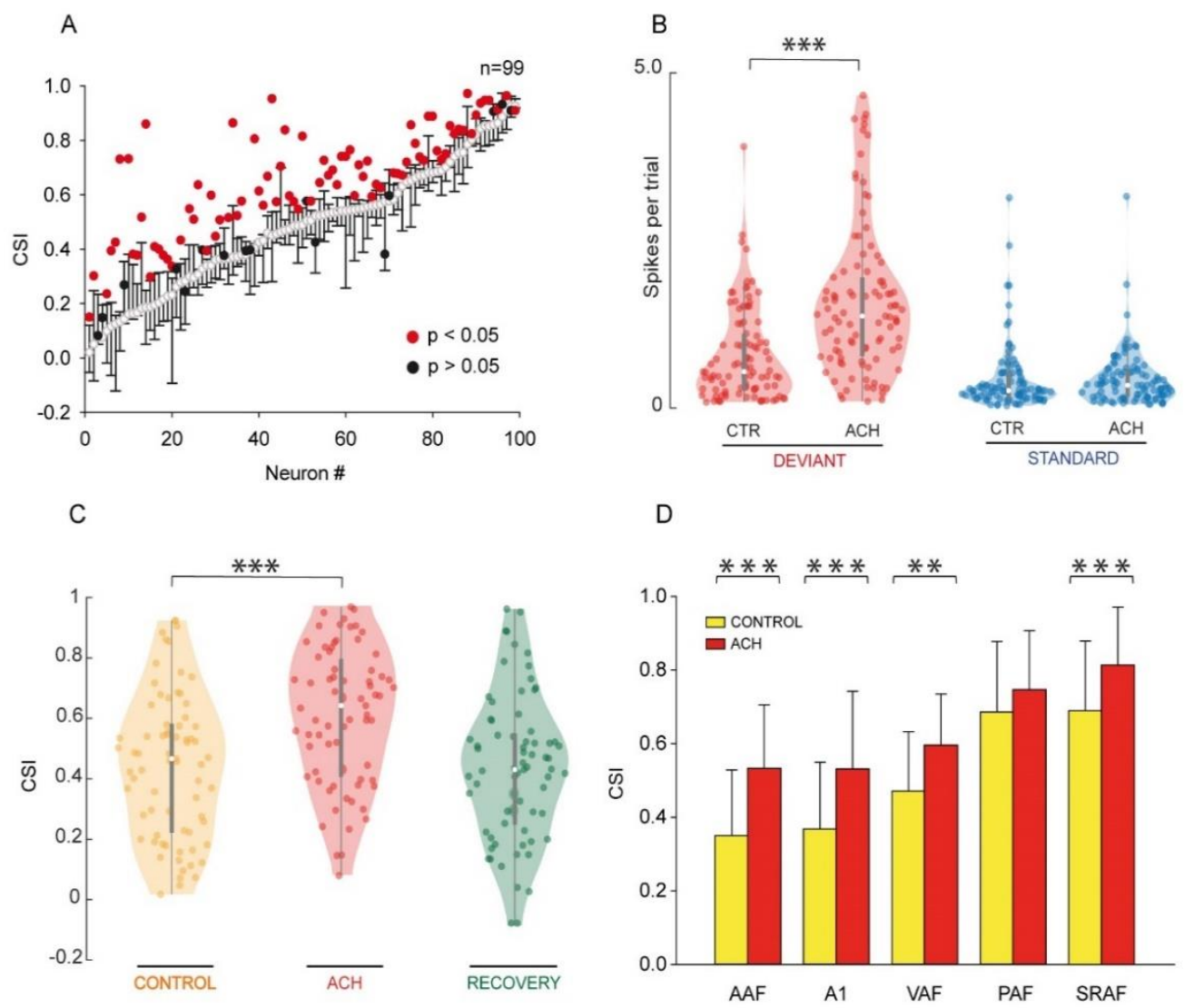

Figure 20: Effect of acetylcholine on SSA levels. (A) Changes on the CSI due to the effect of ACh, for each recorded unit. The units are sorted based on their CSI in the control condition (white dots). In most of the units there was a significant change in CSI after ACh injection (83/99, red dots), while 16 units (black dots) did not change significantly. The vertical bars indicate the $95 \%$ confidence interval for the CSI in the control condition. (B) Violin plots showing the distribution of responses to the deviant (red) and standard tones (blue) in control and ACh conditions. The application of ACh caused a significant increment in the response to deviant tones but not to standard tones. (C) Distribution of CSI values in control, ACh and recovery conditions. The application of ACh caused a significant increase in the CSI, which returned to basal levels afterwards. (D) The average CSI (mean + SD) increased significantly in all lemniscal cortical fields (AAF, A1, VAF) and in SRAF during ACh injection. 
The effect of ACh on SSA in AC and cholinergic antagonist

Two major types of cholinergic receptors are present in the rat AC: muscarinic (mAChR) and nicotinic (nAChR). The nAChR are distributed mainly in layers I and II while mAChR are more widely distributed across layers (Colangelo et al., 2019; Edeline, 2012). To examine whether the effects of ACh were mediated by muscarinic or nicotinic receptors, we recorded from 23 additional neurons before, during and after the microiontophoretic injection of their corresponding antagonists: scopolamine and mecamylamine (Fig. 21A and 21B). Scopolamine significantly reduced the firing rate in response to deviant tones (1.56 \pm 1.33 vs $0.84 \pm 0.97$ spikes per trial; control vs scopolamine; Wilcoxon Signed Rank test, $p<0.001$; Fig. $21 \mathrm{~A}$ ) without affecting the firing rate in response to standard tones ( $0.26 \pm 0.24$ vs $0.29 \pm 0.24$ spikes per trial; control vs scopolamine; Wilcoxon Signed Rank test, $p=0.216$ ). Accordingly, scopolamine reduced SSA levels in 11 of 13 neurons recorded (CSI: $0.63 \pm 0.14$ vs $0.55 \pm 0.15$; control vs scopolamine; Wilcoxon Signed Rank test, $p<0.001)$. Thus, the effect of scopolamine on firing rates and CSI values was opposite to the effect of acetylcholine. By contrast, mecamylamine (Fig. 21B) did not cause significant changes in the firing rates in response to neither the deviant $(0.91 \pm 0.69$ vs 0.87 \pm 0.70 ; control vs mecamylamine; Wilcoxon Signed Rank test, $p=0.91$ ) nor the standard tones $(0.30 \pm 0.23$ vs $0.47 \pm 0.47$ spikes per trial; control vs mecamylamine; Wilcoxon Signed Rank test, $p=0.28$ ). Therefore, mecamylamine did not affect SSA levels (CSI: $0.58 \pm 26$ vs $0.55 \pm 0.27$; control vs mecamylamine; Wilcoxon Signed Rank test, $p=0.43$ ), suggesting the differential effect of ACh on the response to deviant stimuli is may be mediated by muscarinic receptors. 
A

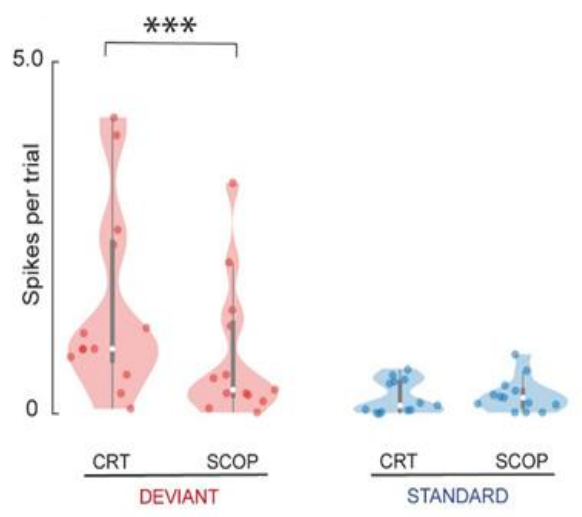

B

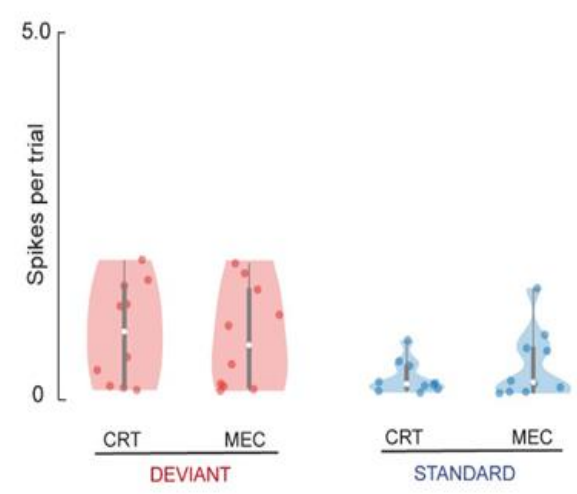

Figure 21: Effect of cholinergic antagonist on firing rate levels (A) The application of the muscarinic antagonist scopolamine (SCOP) caused a significant decrement in the responses to deviant tones, but did not affect the responses to standard tones. (B) In contrast, the application of the nicotinic antagonist mecamylamine (MEC) did not cause changes in the responses to neither deviant nor standard tones. In this and similar figures: ${ }^{*} p<0.05 ;{ }^{* *} p<0.01 ;{ }^{* * *} p<0.001$.

ACh effect on SSA as a function of topographic distribution in auditory cortical layers and fields

Another question in this thesis is whether the effect of ACh is uniformly distributed across AC neurons in different fields and layers. For this, we calculated synthetic maps to show the SSA and spikes per trial levels for deviant and standard responses and later, we studied the distribution of CSI values as a function of recording depth within the $A C$ with and without $A C h$ injection. Figure $22 \mathrm{~A}$ and $22 \mathrm{C}$ shows the CSI and spike count levels for the deviant and standard tones before and after ACh application as well as the difference between the ACh and control conditions. The highest levels of CSI in the control condition (left column) were found in SRAF and PAF. After ACh application (middle column), the increase in CSI and the deviant spike counts were larger in the primary auditory fields (A1, AAF and VAF). By contrast, the ACh injection did not affect the spike 
count for the standard tone (middle column, bottom panel). When the responses during the control condition were subtracted from the ACh condition (right column), it became evident that the largest differences in the CSI were due to spike count increments in response to the deviant tone, as the activity for the standard tone remained largely unchanged. Figure 22D shows the distribution of $\mathrm{CSI}$ values as a function of recording depth within the $\mathrm{AC}$ under control conditions (yellow) and after the ACh injection (red). While CSI values were not different across layers in the control condition, they increased in all recorded layers (II to $\mathrm{VI}$ ) in response to ACh (layer II: $0.51 \pm 0.26$ vs $0.65 \pm 0.23$, control vs $A C h, p=$ 0.001 ; layer III: $0.45 \pm 0.22$ vs $0.57 \pm 0.21 ; p<0.001$; layer IV: $0.43 \pm 0.23$ vs 0.62 $\pm 0.22, p<0.002$; layer V: $0.49 \pm 0.23$ vs $0.63 \pm 0.22, p<0.001$; layer Vl: $0.52 \pm$ 0.23 vs $0.69 \pm 0.21 ; p<0.001)$. The effect of ACh on the CSI across cortical layers was similar for lemniscal (Fig. 22F, green dots) and non-lemniscal (Fig. $22 F$, grey dots) fields (Mann-Whitney test, $p=0.06$ ). Similarly, we found no differences in the CSI between supra- and infragranular layers of the AC (MannWhitney test, $p=0.17)$. In summary, all these data demonstrate that ACh has a global and distributed effect on SSA regardless of the field or layer within the AC (Fig. 22D). In summary, all these results demonstrate that ACh has a global and distributed effect on SSA regardless of the field or layer within the AC. 

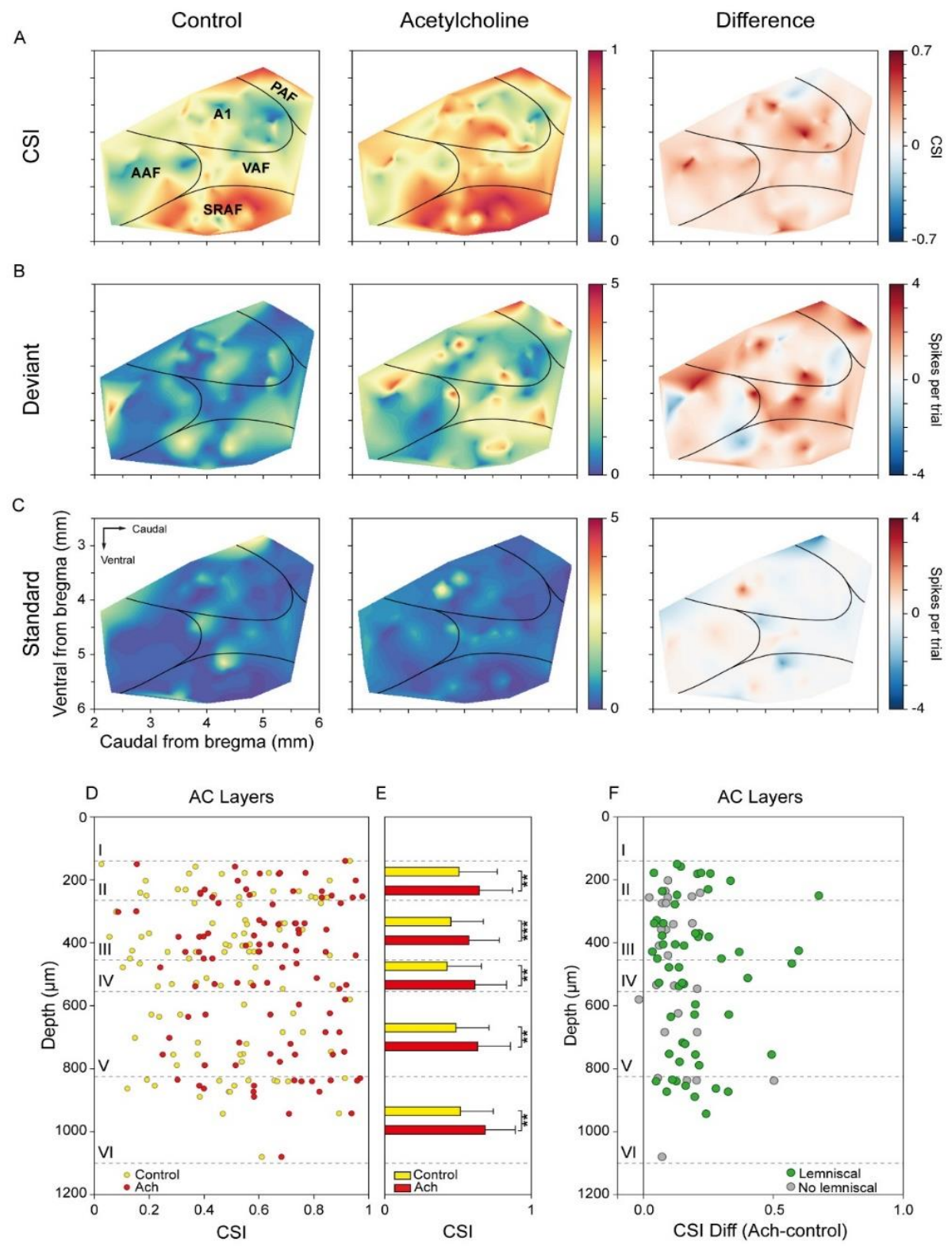

Figure 22: Anatomical localization of CSI and responses to the deviant and standard tones. Distribution of CSI (A) and responses (spikes per trial) for deviant (B) and standard tones (C), before and after ACh application, as well as the difference between both conditions. The highest levels of CSI are found in non-lemniscal fields, (SRAF and PAF). The application of ACh increased the response to the deviant tones throughout the AC but did not affect the responses to standard tones. (D) CSI levels of the recorded units according to their recording depth. The dashed lines indicate the approximate limits of the cortical layers. Yellow dots indicate the CSI in the control condition, while red dots indicate the corresponding CSI under the influence of ACh. We did not have any recordings that could be unambiguously located in layer I. (E) Average (mean + SD) CSI in control (yellow) and ACh condition (red), for each cortical layer. (F) Distribution of the difference between the levels of CSI obtained in the control and the ACh conditions (ACh-Control) for lemniscal (green) and non-lemniscal units (grey). Units with non-significant changes are excluded. 
ACh affects neuronal mismatch, increasing prediction error but not repetition suppression in cascade and many-standard paradigms.

Having studied cholinergic modulation is SSA levels and the firing rate discharge to the deviant and standard tones, our next step was to determinate how ACh modulates the neuronal mismatch (iMM), prediction error (iPE) and repetition supression (iRS) indices. For this, we recorded 65 additional lemniscal and nonlemniscal units, using the many-standard and cascade control sequences previously published (Carbajal and Malmierca, 2018; Parras et al., 2017; Ruhnau et al., 2012; Valdés-Baizabal et al., 2020) First, we recorded the spike counts in response to the control (CAS), deviant (DEV), and standard (STD) stimuli (Fig 23 A-B). ACh led to significant differences only in the DEV responses in the lemniscal fields (Wilcoxon Signed Rank test, $p=0.013$ ). While there was a trend, the effect was not significant in the non-lemniscal fields (Wilcoxon Signed Rank test, $p=0.066$ ), probably because the $D E V$ responses were already strong during the control condition. Next, using the normalized CAS, DEV and STD responses (see Material and Methods), we computed the indexes iMM, iPE and iRS before and after the ACh injection in lemniscal (Figure 23C, iMM: $0.58 \pm 0.29$ vs $0.75 \pm$ 0.17 ; iPE: $0.25 \pm 0.41$ vs $0.49 \pm 0.32$; iRS: $0.31 \pm 0.32$ vs $0.28 \pm 0.25$; control vs ACh, respectively) and non-lemniscal fields (Figure 17D, iMM: $0.56 \pm 00.29$ vs $0.75 \pm 0.17$; iPE: $0.22 \pm 0.40$ vs $0.44 \pm 0.40$; iRS: $0.31 \pm 0.27$ vs $0.27 \pm 0.26$ ) Significant effects of ACh administration were found for iMM and iPE, both in lemniscal (iPE: $p=0.006$; iMM: $p=0.005$; Wilcoxon Signed Rank test; Fig 23C) and non-lemniscal areas (iPE: $p=0.015$; iMM: $p=0.026$; Wilcoxon Signed Rank test; Fig 23D), while iRS remained unchanged (lemniscal iRS: $p=0.41$; nonlemniscal iRS: $p=0.53$; Wilcoxon Signed Rank test). Next, we compared the 
changes that occurred between the control and ACh conditions for the iMM, iPE and iRS respectively, both for the lemniscal (grey dots) and non-lemniscal fields (colored dots, Figure 23E-G). iMM and iPE values tend to lie above the main diagonal while the iRS values are distributed throughout the plot, i.e., ACh increased the magnitude of the iMM and this scaling is mostly due to an increase in the iPE. Finally, and to further demonstrate that the scaling effect of ACh is on iPE and not on iRS we performed a regression analysis to examine the correlation between changes on the iMM and changes in either the iPE or the iRS. The linear model demonstrated a direct relationship between iMM and iPE changes (Fig. 23H) while no apparent relationship existed between iMM and iRS changes (Fig. 23I).

The models for the lemniscal fields (Fig. 23H, colored dots and lines) were:

$$
\begin{aligned}
& i P E_{\text {change }}=0.12+0.75\left(i M M_{\text {change }}\right) ; R^{2}=0.23 \\
& i R S_{\text {change }}=-0.02-0.04\left(i M M_{\text {change }}\right) ; R^{2}=8.44 e-4
\end{aligned}
$$

While the models for the non-lemniscal fields (Fig. 23I, grey dots and lines) were:

$$
\begin{aligned}
& i P E_{\text {change }}=0.13+0.64\left(i M M_{\text {change }}\right) ; R^{2}=0.21 \\
& i R S_{\text {change }}=-0.02-0.10\left(i M M_{\text {change }}\right) ; R^{2}=6.18 e-3
\end{aligned}
$$



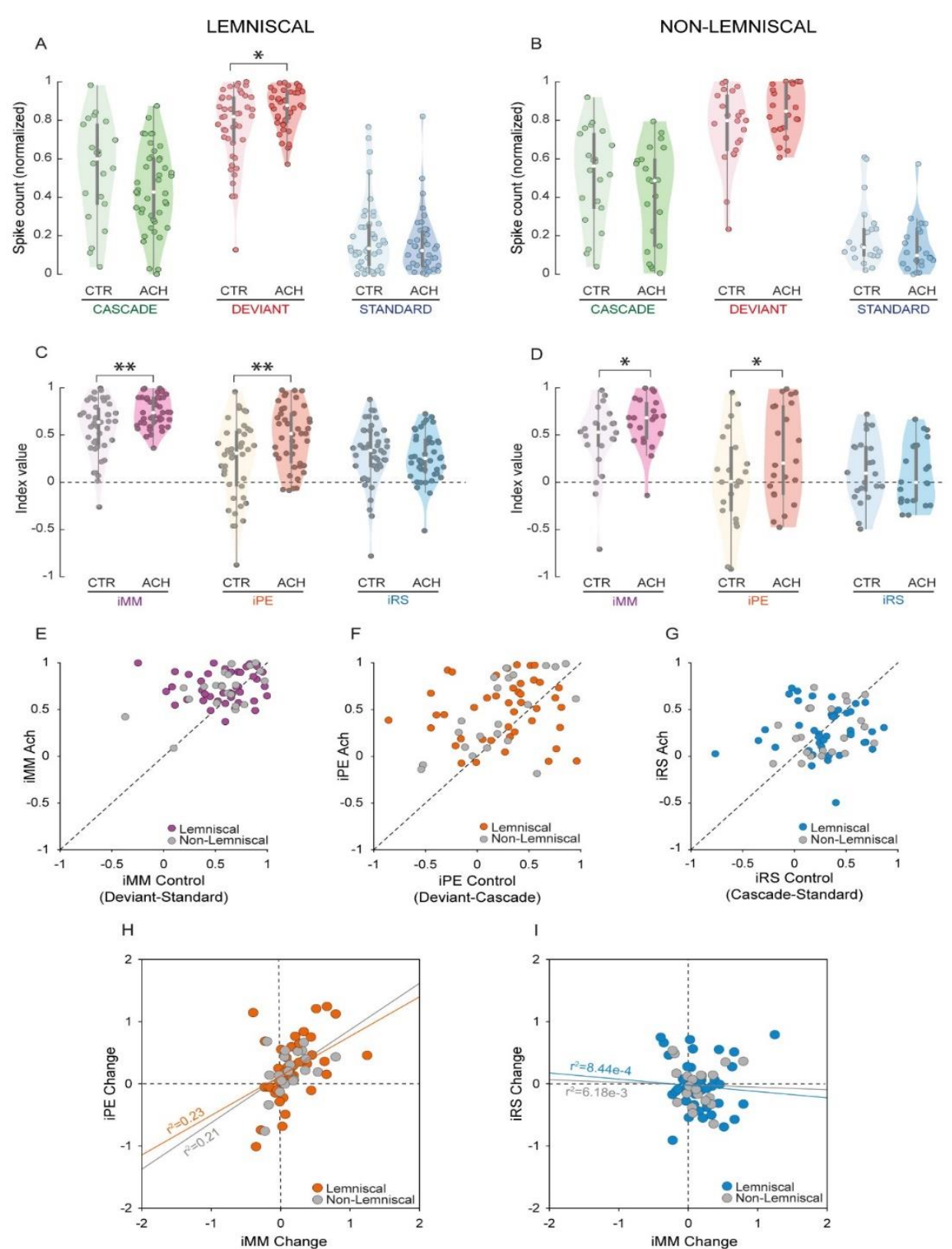

Figure 23: Predictive coding indices in lemniscal and nonlemniscal areas. The application of ACh increased the normalized spike counts in response to deviant stimuli (red) in lemniscal (A) but not in non-lemniscal areas (B), and had no effect on the responses to neither the standard tones (blue) nor the cascade controls (green). In consequence, the neuronal mismatch (iMM, purple) and prediction error indexes (iPE, orange) increased significantly in both lemniscal (C) and non-lemniscal areas (D), but not the repetition suppression index (iRS, cyan). (E, F and G) Scatter plots showing the effect of ACh for each individual unit on $\mathrm{iMM}(\mathrm{E}), \mathrm{PPE}(\mathbf{F})$ and $\mathrm{R} S$ (G), from lemniscal (coloured dots) or non-lemniscal areas (gray dots). The changes of iPE due to the application of ACh $(\mathbf{H})$ were more correlated with the changes of IMM than the changes of iRS. 
In summary, the principal conclussions of this work include: 1) ACh produced a significant increment in SSA levels, in neurons from lemniscal and non-lemniscal cortical fields; 2) SSA increments were fundamentally produced by an enhancement in the firing rate in response to the deviant tones. The firing rate in response to the standard tones did not vary significantly; 3) the firing rate in response to the deviant tones decreased significantly with the application of muscarinic cholinergic antagonists (scopolamine), but not with nicotinic antagonists (mecamylamine) and 4) ACh increases the mismatch negativity and prediction error in lemniscal and non-lemniscal areas, but has no effect on repetition suppression. 


\section{Section III: Effect of behaviorally relevant sounds in deviant detection and prediction error responses}

In this last section, we studied the effect of behaviorally relevant sounds in saliency detection and prediction error response in trained rats. The results of this study are published in Morado-Díaz et al. (2020) (in preparation). In order to show that rats had normal hearing through all the behavioral experiment, ABR-tests were conducted in all animals before the training start and at the end of the experiments (Fig. 24D). The analysis showed no significant differences between experimental groups $\left(F=5.56 \times 10^{-8}\right)$, before and after the training $\left(F=3.66 \times 10^{-9}\right)$, or comparing responses recorded from the left and right ears $\left(F=3.09 \times 10^{-8}\right)$.

Here, we trained 16 freely moving rats using different variants of an auditory discrimination task to check if repeated exposure to relevant and/or irrelevant sounds show long-term effects on the representation of those sounds in A1 neurons. In the first phase of training, two pure tones were separated by 0.5 octaves and were presented at a rate of $0.5 \mathrm{~Hz}$. In order to receive a food reward, animals were asked to activate the nose-poke device in the operational chamber when a deviant tone appeared, and to ignore the standard tones (Fig. 24A, Oddball training; 24E). Responses were categorized as correct responses (HITS) when the animal nose-poked in response to a deviant tone; false alarms (FA) if the animal responded to a standard tone; correct rejections (CR) if the animal showed no response after a standard tone; and missed responses (MISS) when the animal failed to response to deviant tones. Thus we evaluated the general performance task using the d'sensitivity index (Fig. 24B). Animals were randomly 
assigned to 4 different groups (4 rats each). For each group, we used different pairs of sound frequencies, but the standard tone was kept constant per group though all the sessions and the variants of the task (Table 2). Once rats showed a performance score of $d^{\prime} \geq 1$ for 3 consecutive sessions (Fig. 24C), animals were ready for data collection using a set of different variants of the auditory discrimination task (Fig. 25).

\begin{tabular}{|l|l|l|}
\hline GROUPS & FREQUENCIES (kHz) & NUMBER OF ANIMALS \\
\hline 1 & $4.8-6.7$ & 4 \\
\hline 2 & $5.7-8.0$ & 4 \\
\hline 3 & $6.7-9.5$ & 4 \\
\hline 4 & $8.0-11.3$ & 4 \\
\hline Naïve & Untrained & 4 \\
\hline
\end{tabular}

Table 2: pairs of frequencies used for the 4 experimental groups 
A

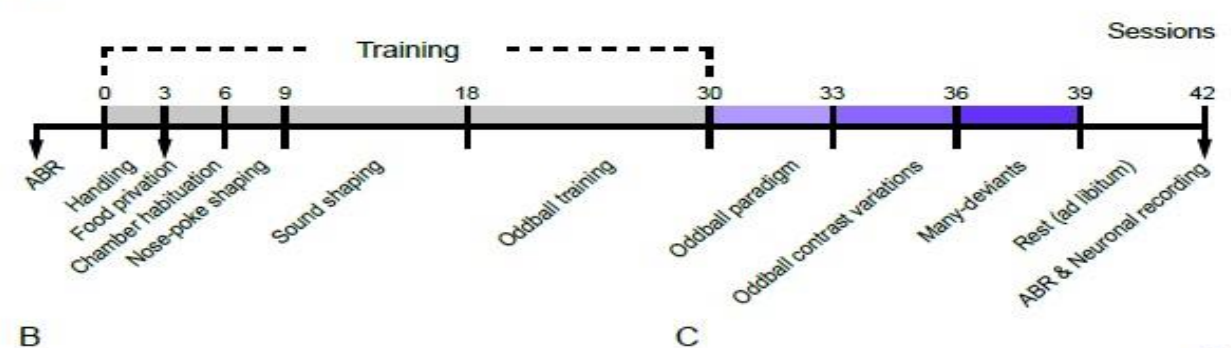

B


D
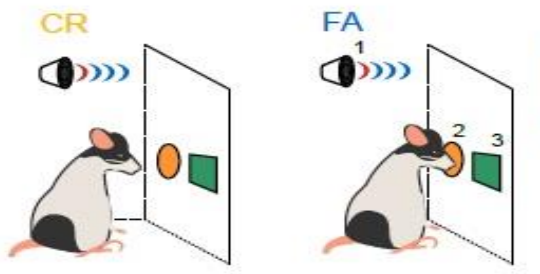

1. Speaker

2. Nose pok

Deviant tone

, Standard tones
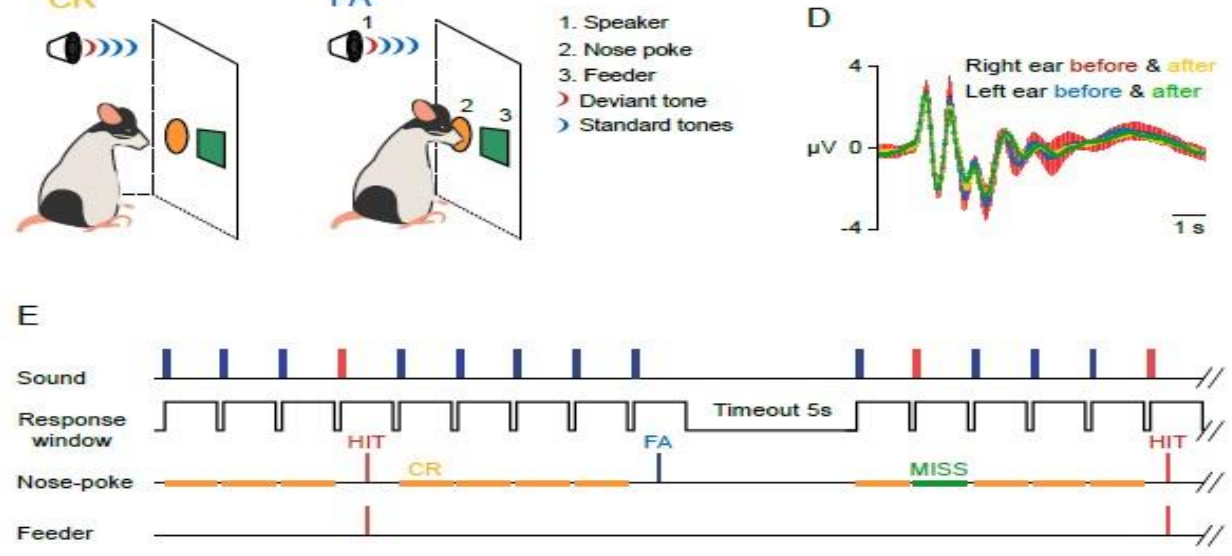

Figure 24: Experimental design. (A) Timeline of training/test sessions across the course of the experiment. (B) Schematic representation of possible responses of the animals inside the operational chamber quantified during performance of behavioral sessions equipped with a speaker (1) to display sound sequences. Nose-poke (2) responses in the response window after a deviant tone (red wave) rewarded with one pellet (3) were considered as "hits" (HIT). The absence of response to deviant tone were computed as "missed responses" (MISS). "False alarms" (FA) were responses to a standard tone (blue wave), and "correct rejections" were absence of response after a standard tone (CR). (C) Learning curve representing mean d' values (red line) \pm S.E.M. (pink area) computed for trained rats in the las 6 sessions of the training oddball phase. Rats completed training when they reached criterion performance ( $\left.d^{\prime} \geq 1\right)$ for 3 consecutive sessions (asterisk). (D) Mean \pm S.D.M. auditory brainstem responses (ABR) recorded at the initial and final sessions (before and after, respectively) for both ears (right, red and yellow; left, blue and green). No significant differences were found before re after, between right re left responses or between groups (three-way ANOVA test, $P>0.001)$. (E) Temporal structure of behavioral trials. Note that every FA was followed by $5 \mathrm{~s}$ of timeout 
Behavioral responses to the oddball paradigm.

Data was acquired for 3 sessions (in 3 consecutive days), in which animals were presented with the corresponding pair of tone frequencies (these frequencies varied with the group they belong to, see Table 2). All trained rats showed a similar pattern of responses (Fig. 26A) with a high percentage of hits (mean \pm SEM: $64.58 \pm 2.03 \%)$ and correct rejections (89.22 $\pm 0.91 \%)$, and a low level of false alarms (10.78 \pm 0.91\%) and missed responses (36.42 $\pm 2.03 \%)$. Interestingly, rats from group $5.7 / 8.0 \mathrm{kHz}$ (as standard/deviant tone frequencies during training, respectively) showed a higher percentage of hits and lower percentage of miss responses others trained groups (two-way ANOVA tests for repeated measures of \%HIT, \%CR, \%FA and \%MISS, P<0.001; Holm-Sidak method for multiple comparisons, $\mathrm{P}<0.05)$. By contrast, naïve animals completed the task with a very low percentage of hits $(0.24 \pm 0.13)$ and false alarms $(0.25 \pm$ $0.05)$ responses and a high level of correct rejections $(99.75 \pm 0.05)$ and miss $(99.76 \pm 0.13)$ responses. Consequently, all trained rats showed an average $d$ ' value of $1.72 \pm 0.07(1.30 \pm 0.06,1.97 \pm 0.21,1.84 \pm 0.20$ and $1.79 \pm 0.10$ for groups $4.8-6.7 \mathrm{kHz}, 5.7-8.0 \mathrm{kHz}, 6.7-9.5 \mathrm{kHz}$ and $8.0-11.3 \mathrm{kHz}$, respectively), i.e., larger than the discrimination threshold $\left(d^{\prime}=1\right)$, and significantly different to the naïve group ( $d^{\prime}=0.20 \pm 0.06$; two-way ANOVA test for repeated measures, $F_{\text {Group }}=26.60, F_{\text {Session }}=5.91, F_{\text {Group }} \times$ Session $\left.=1.64, P<0.001\right)$. 


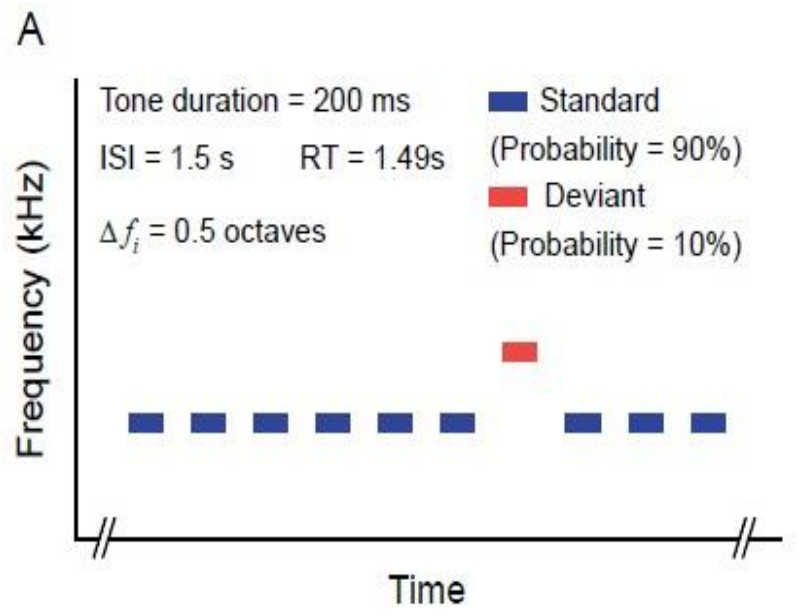

B

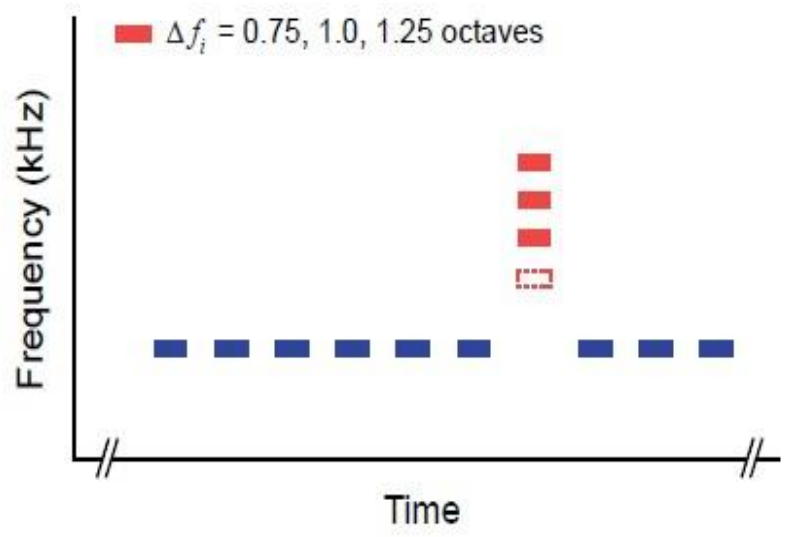

C

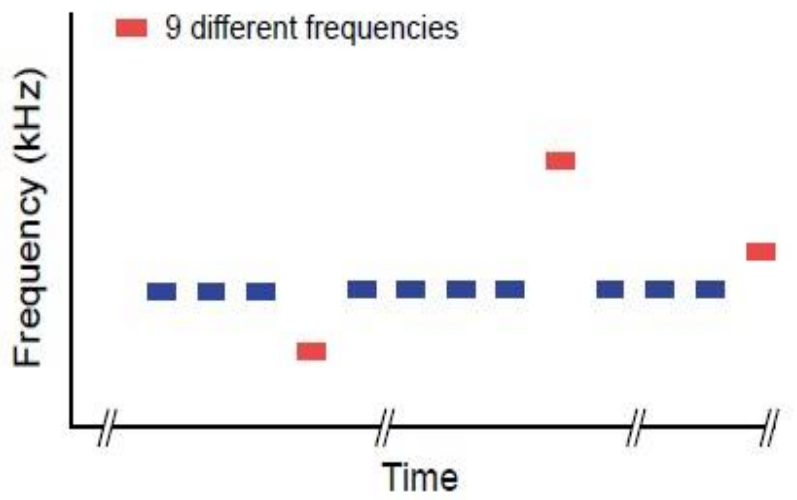

Figure 25: Paradigm sequences. (A) Oddball sequence task consisted in high probability standard tones (90\%; in blue) randomly interrupted by low probability deviant tones (10\%; in red) and 0.5 octaves in frequency contrast $\left(\Delta \mathrm{f}_{\mathrm{i}}\right)$. Tone duration, interstimulus interval (ISI) and response time (RT) were $200 \mathrm{~ms}, 1.5 \mathrm{~s}$ and $1.49 \mathrm{~s}$, respectively. (B) Deviant generalization task was a similar paradigm, but in this case, the frequency contrast between standard/deviant tones varied in 3 different sessions, being 0.75 octaves in the first, 1.0 octaves in the second and 1.25 octaves in the third. (C) Many-deviant task consisted in a many deviants sequence made of several blocks of the oddball paradigm as $\mathrm{A}$, where every 10 stimuli, the sound frequency of a deviant tone was randomly changed from 9 possibilities of different tones, and the standard sound was maintained throughout the entire sequence.

In order to test if rats reached a plateau in the discrimination rate on day one or they were still learning the task and improving their performance, we compared the $d^{\prime}$ 'values obtained in the three consecutive sessions. The comparison of averaged $d$ 'values across days revealed significant differences between the first 
and the third session $(1.48 \pm 0.12,1.75 \pm 0.16$ and $1.94 \pm 0.18$ for sessions 1,2 and 3 , respectively). Thus, the data suggest that although animals started the behavioral analysis once they had reached a high discrimination value, they were still learning the task and improving their performance (Fig. 26B).

A


Figure 26: Behavior responses to oddball sequence task. (A) Violin plots showing the distribution of averaged percentage (of 3 sessions/animal) of hits after a deviant tone (HIT, in red), correct rejection of standard tones (CR, in yellow), false alarms (FA, in blue) and missed responses after a deviant tone (MISS, in green) performed by untrained (Naïve) and trained animals distributed in different groups (4 rats/group) stablished in function of standarddeviant tone frequencies used during training and presented in these oddball paradigms $(4.8-6.7 \mathrm{kHz}, 5.7-8.0,6.7-9.5 \mathrm{kHz}, 8.0-11.3 \mathrm{kHz})$. The thick grey bars expand from the first (Q1) to the third quartile (Q3), and the whiskers show the range of lower and higher adjacent values. Significant differences with respect to naïve group and concrete groups are indicated by * and numbers (referring that different group), respectively (two-way ANOVA test for repeated measures per category, $\mathrm{P}<0.001$; Holm-Sidak method for multiple comparisons, $p<0.05$ ). (B) Bar chart showing d'values (mean $\pm S E M$ of all animals per group) obtained for sessions 1,2, 3 and the averages of them. Note that $d$ ' values of trained animals were upper 1 , the discrimination threshold stablished (dashed line). ${ }^{*}$ and - represent significant differences with respect to naïve group and sessions, respectively (two-way ANOVA test for repeated measures, $\mathrm{P}<0.001$; Holm-Sidak method for multiple comparisons, $\mathrm{p}<0.05$ ). 
Frequency contrast impact on behavioral responses to oddball paradigm

In order to test if trained rats were better discriminating deviant tones as a function of the frequency contrast relative to the standard tone, we presented a variant of the oddball paradigm with the same standard tone but varying the sound frequency of the deviant tone in 0.25 octave steps from the original deviant frequency (i.e., $0.75,1.0$ and 1.25 octaves; Fig. 25B). Results were obtained after 3 consecutive sessions, presenting a frequency contrast of 0.75 octaves in the first, 1.0 octaves in the second, and 1.25 octaves in the third one. The performance in all trained groups was similar to the previous task, with high percentage of hits and correct rejections, and low levels of false alarms and missed responses in the 3 frequency contrasts used (Fig. 27A). Thus, if the frequency contrast between standard and deviant was 0.75 octaves, the mean \pm SEM percentage values for hits, correct rejection, false alarms and miss responses for all rats in all trained groups were $73.85 \pm 6.26 \%, 93.30 \pm 1.58 \%$, $6.70 \pm 1.58 \%$ and $26.15 \pm 6.26 \%$, respectively. The values for a frequency contrast of 1.0 octave were $76.91 \pm 5.75 \%, 94.34 \pm 1.44 \%, 5.66 \pm 1.44 \%$ and $23.09 \pm 5.75 \%$, and for 1.25 octaves were $79.34 \pm 5.41 \%, 95.13 \pm 1.49 \%, 4.87 \pm$ $1.49 \%$ and $20.66 \pm 5.41 \%$ as percentages of hits, correct rejections, false alarms and miss responses, respectively (two-way ANOVA tests for repeated measures of \%HIT, \%CR, \%FA and \%MISS, with "Group" and "Contrast" as factors, $\mathrm{P}<0.001$; Holm-Sidak method for multiple comparisons, $\mathrm{P}<0.05)$. When the responses of these trained animals were computed as d'values, they were all larger than 1 (Fig. 27B), and progressively increased as a function of the frequency contrast used $(2.31 \pm 0.28,2.48 \pm 0.24$ and $2.68 \pm 0.30$ for $0.75,1.0$ and 1.25 octaves, respectively; Fig. $27 \mathrm{C}$ ). Note that mean \pm SEM $d$ ' of trained 
animals using a frequency disparity of 0.5 octaves (oddball paradigm; Fig. 26) was $1.72 \pm 0.07$, a value significantly lower than presenting larger discrepancies in tone frequency (two-way ANOVA test for repeated measures, $\mathrm{P}<0.001$; HolmSidak method for multiple comparisons, $\mathrm{P}<0.05)$. Thus, this data suggest that rats discriminate better deviant tones that are farther away than the frequency of the standard tone. 


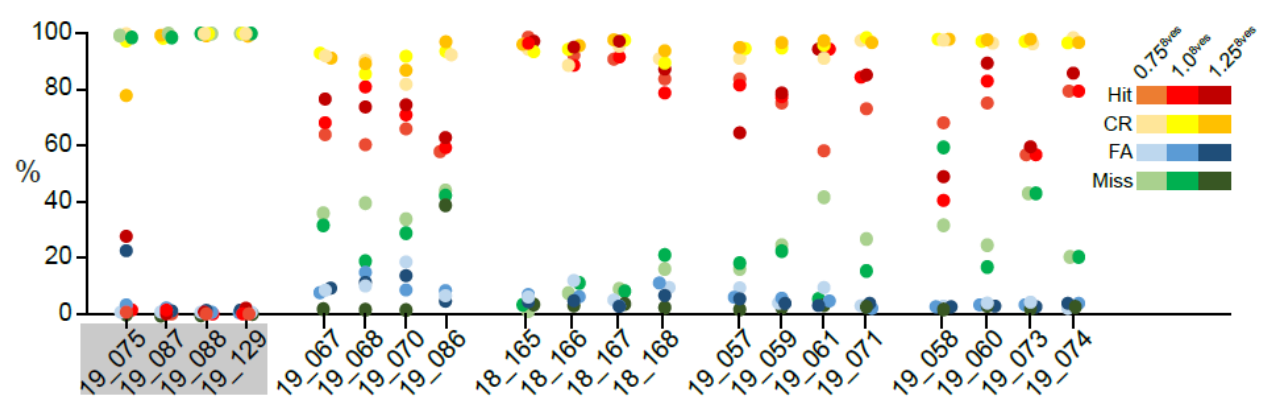

B

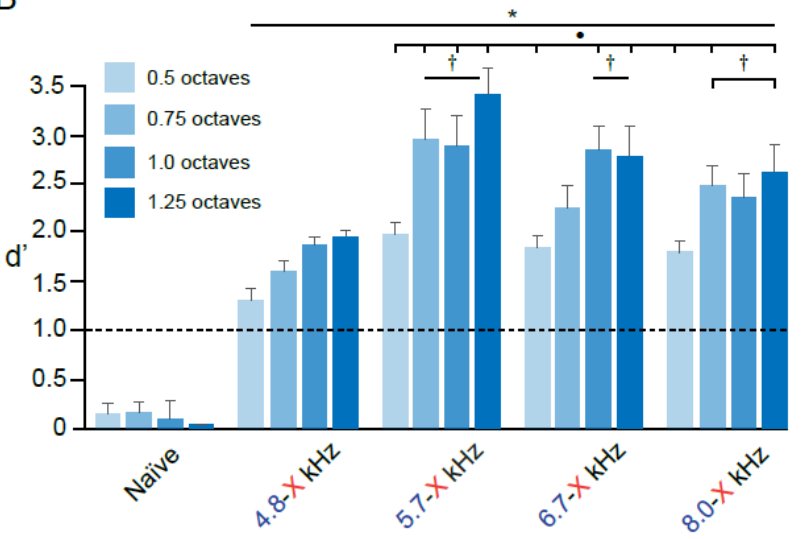

C

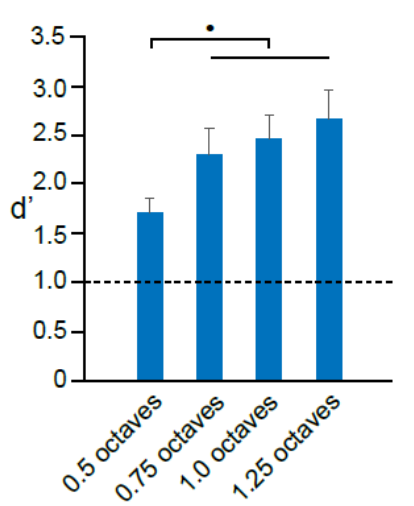

Figure 27: Behavior responses to oddball sequence task varying frequency contrast. (A) Representation of percentage of hits after a deviant tone (HIT, in red), correct rejection of standard tones (CR, in yellow), false alarms (FA, in blue) and missed responses after a deviant tone (MISS, in green) performed by untrained (Naïve; highlighted in grey) and trained animals distributed in different groups (4 rats/group) stablished in function of standarddeviant tone frequencies (contrasted in 0.5 octaves) used during training (from left to right, $4.8-6.7 \mathrm{kHz}, 5.7-8.0,6.7-9.5 \mathrm{kHz}, 8.0-11.3 \mathrm{kHz}$ ). Frequency contrast was varied to 0.75 (lighter colors), 1.0 (medium colors) and 1.25 (darker colors) octaves modifying deviant tone frequency. (B) Bar chart showing $d^{\prime}$ 'values (mean \pm SEM of all animals per group) obtained with tone frequency contrasts of $0.5,0.75,1.0$ and 1.25 octaves. Note that $d$ ' values of trained animals were upper 1 , the discrimination threshold stablished (dashed line). * $\cdot$ and † represent significant differences with respect to naïve group, respect to same contrast of other group and respect to contrasts of 0.5 octaves, respectively (two-way ANOVA test for repeated measures, $P<0.001$; HolmSidak method for multiple comparisons, $P<0.05$ ). (C) Bar chart representation of $d$ ' values (mean \pm SEM of all trained animals) calculated for the different standard/deviant contrasts tested $(0.5,0.75,1.0$ and 1.25 octaves). Significant differences respect contrast of 0.5 octaves are indicated by $\bullet$ (two-way ANOVA test for repeated measures, $P<0.001$; Holm-Sidak method for multiple comparisons, $P<0.05)$. 


\section{Responses to many-deviants sequence}

The last three sessions were performed under another variant of the auditory discrimination task that consisted in an oddball paradigm where frequency contrast between standard and deviant frequencies was randomly varied from 9 possibilities while the standard tone remained constant (Fig. 25C). The main goal of this variant was to confirm that rats were responding to any deviant tone violating the regularity stablished by the standard sound. As in previous settings, all trained rats completed the task with high percentage of hits $(68.96 \pm 8.21 \%)$ and correct rejections $(90.74 \pm 0.96 \%)$ responses, and low levels of false alarms $(9.26 \pm 0.96 \%)$ and miss $(31.04 \pm 8.21 \%)$ responses. Results were compared with one-way ANOVA tests for \%HIT, \%CR, \%FA and \%MISS, with "Group" as factor $(\mathrm{P}<0.001$; Holm-Sidak method for multiple comparisons, $\mathrm{P}<0.05$; Fig. 28A) and show that rats are generalizing the deviant tone to any frequency that deviates from the standard repeating sound. Even though $d^{\prime}$ 'values were above 1 for all animals (Fig. 28B), it is clear that, except for group 4.8 -Y kHz that showed a $\mathrm{d}^{\prime}=1.71 \pm 0.09, \mathrm{~d}^{\prime}$ values decreased progressively from group $5.7-\mathrm{Y} \mathrm{kHz}$ to group 8.0-Y kHz (group 5.7-Y kHz: $2.55 \pm 0.18$; group 6.7-Y kHz: $2.25 \pm 0.11$; and group 8.0-Y kHz: $1.20 \pm 0.17$ (one-way ANOVA test, $\mathrm{P}<0.001$; Holm-Sidak method for multiple comparisons, $\mathrm{P}<0.05$; Fig. 28B). Interestingly, the average frequency contrast between the standard tones in group 4.8-Y kHz, group 5.7-Y $\mathrm{kHz}$, group 6.7-Y kHz and group 8.0-Y $\mathrm{kHz}$ and the corresponding average deviant tones for these groups also decreased (1.2, 0.9, 0.8 and 0.7 octave differences group, 5.7-Y kHz, 6.7-Y kHz, y 8.0-Y kHz; Fig. 28C). These results are consistent with the results presented in the previous section (compare figures $27 \mathrm{C}$ and $28 \mathrm{C}$ ) and suggest that the decrease in discrimination in these groups 
may be correlated with the fact that the mean frequency distance between deviant and standard sounds is smaller in these groups.

A

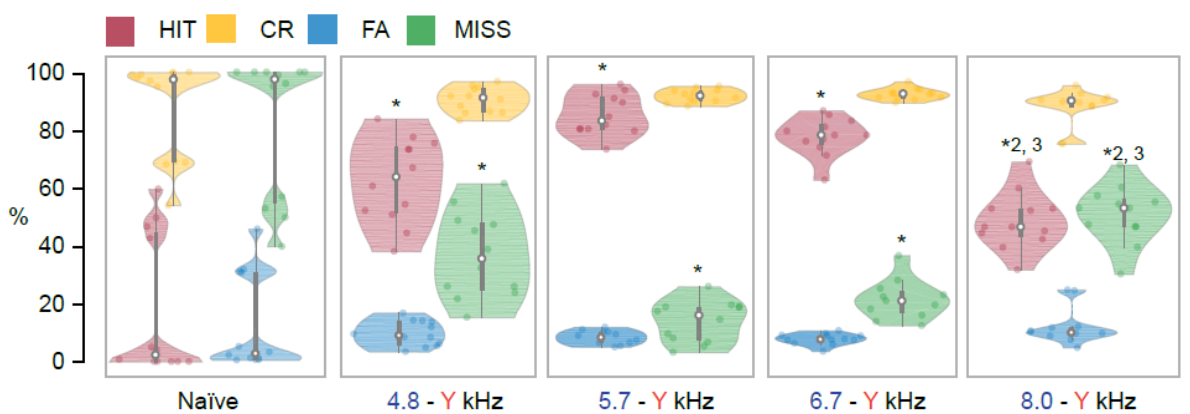

B

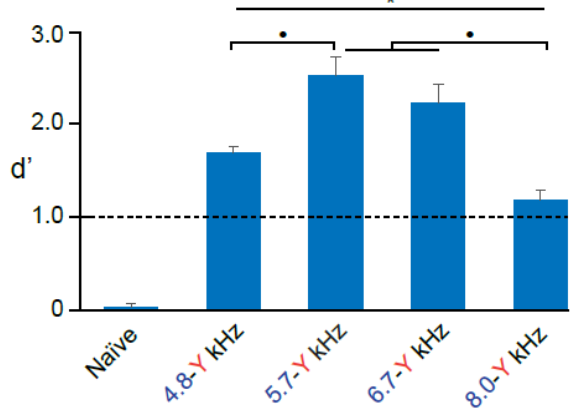

C

Figure 28: Behavior responses to many-deviants sequence task. (A) Violin plots showing the distribution of averaged percentage (of 3 sessions/animal) of hits after a deviant tone (HIT, in red), correct rejection of standard tones (CR, in yellow), false alarms (FA, in blue) and missed responses after a deviant tone (MISS, in green) performed by untrained (Naïve) and trained animals distributed in different groups (4 rats/group) stablished in function of standarddeviant tone frequencies used during training $(4.8-6.7 \mathrm{kHz}, 5.7-8.0,6.7-9.5$ $\mathrm{kHz}, 8.0-11.3 \mathrm{kHz}$ ). In this paradigm, every deviant tone varied randomly in frequency between 9 possibilities $(4.0,4.8,5.7,6.7,8.0,9.5,11.3,13.5,16.0$ and $19.0 \mathrm{kHz}$ ) maintaining unchanged the standard frequency from the training (excluded from the deviant possibilities). The thick grey bars expand from the first (Q1) to the third quartile (Q3), and the whiskers show the range of lower and higher adjacent values. Significant differences with respect to naïve group and concrete groups are indicated by * and numbers (referring that different group), respectively (one-way ANOVA tests for each category, $P<0.001$; HolmSidak method for multiple comparisons, $P<0.05)$. (B) Bar chart showing $d$ ' values (mean \pm SEM of 3 consecutive sessions for all animals per group). Note that $d$ ' values of trained animals were upper 1 , the discrimination threshold stablished (dashed line). ${ }^{*}$ and $\bullet$ represent significant differences with respect to naïve group and between trained groups, respectively (one-way ANOVA test, $P<0.001$; Holm-Sidak method for multiple comparisons, $P<0.05)$. (C) Bar chart representation of the average frequency contrast \pm SEM between the standard tones in trained groups. 
Behavioral effects on neural responses in auditory cortex.

Once the different auditory paradigms and training protocols were finalized, we recorded the electrophysiological responses in A1 to show whether the frequencies at which they were trained had a greater deviant response (compared to control frequencies) and if they also exhibited higher levels of prediction error. For this, we recorded 68 extracellular responses (28 single- and 40 multiunit) after the behavioral experiments were concluded in 17 rats (4 from group 4.8-6.7 kHz, 3 from group 5.7-8.0 kHz, 4 from group 6.7-9.5 kHz, 3 from group 8.0-11.3 kHz, and 3 from naïve group). Recordings were made at $300-$ $1000 \mu \mathrm{m}$ depth, thus they included layers III-V. At each recording site we recorded the frequency response area for each animal and look for neurons whose best frequency matched the frequencies used in the training protocol $( \pm 0.3$ octaves). Thus, for the 68 extracellular recording we collected a total of 123 pairs of frequencies. After isolation of a single- or multiunit cluster, we recorded their responses to the classical oddball paradigm and cascade paradigm (Parras et al., 2017; Ruhnau et al., 2012).

The firing rate response to the deviant tone showed significantly larger responses in comparison to the standard tone, for untrained (14.14 \pm 3.79 vs. $3.41 \pm 0.51$ spikes/s, respectively) and trained frequencies (17.80 \pm 4.74 vs. $3.35 \pm 0.37$ spikes/s, respectively). Similarly, neuronal responses in naïve animals also showed a significantly higher firing rate to a tone as deviant $(16.11 \pm 5.17)$ as compared to the same tone as standard $(2.26 \pm 0.38$; two-way ANOVA test, $P<0.05$; Holm-Sidak method for multiple comparisons, $P<0.05)$. 
Then, to determine if training on the auditory discrimination task affected the level of SSA, we tested if there was any difference in CSI (common SSA index) between a pair of frequencies used and not used in training. We observed that the CSI values obtained in trained animals for pairs of frequencies used in the behavior protocol were significantly larger than the CSI values obtained for frequencies not used in the behavioral task $(0.62 \pm 0.05$ and $0.54 \pm 0.08$, respectively; paired t-test, Mann-Whitney $U$ method, $P<0.05$; Fig. 29, all responses), while no significant differences were noted for the naïve group ( 0.26 \pm 0.09 and $0.21 \pm 0.10$, respectively; paired t-test, Mann-Whitney $U$ method, n.s.; Fig. 6, naïve). This finding was significant in 3 of the 4 groups and consistent across all 4 groups (Fig. 29).
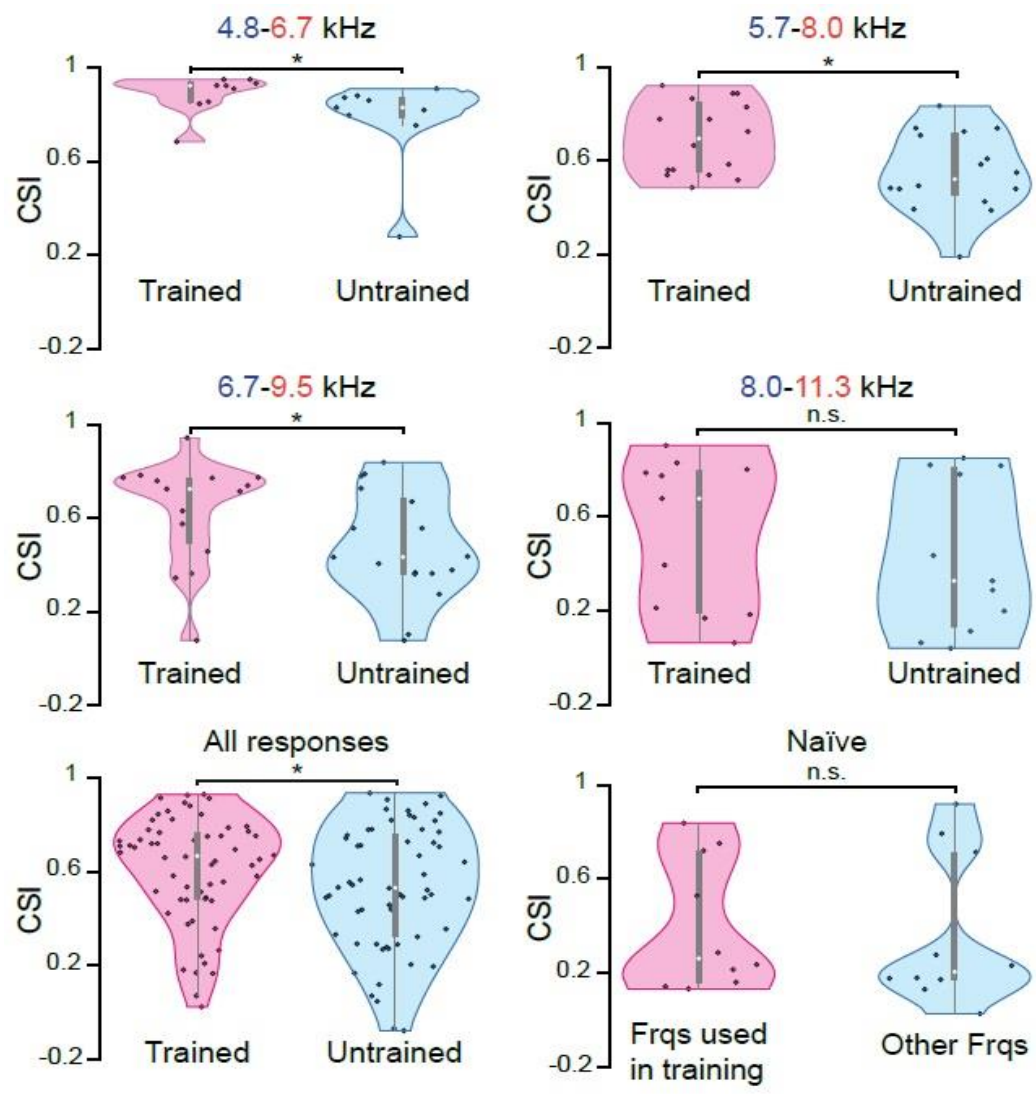
Figure 29: Behavioral training effect on neuronal SSA. (A). Violin plots showing the common SSA index (CSI) computed for neuronal responses to tone frequencies used in the behavioral training (Trained, in purple) and others new (Untrained, in blue) recorded from neuronal units of primary auditory cortex units $(68 ; 123$ pair of tones) recorded in response to oddball paradigms from 3 not trained (Naïve) and 14 trained animals distributed in different groups (4 rats/group) stablished in function of standard-deviant tone frequencies used during training $(4.8-6.7 \mathrm{kHz}, 5.7-8.0,6.7-9.5 \mathrm{kHz}, 8.0-11.3 \mathrm{kHz})$. The thick grey bars expand from the first (Q1) to the third quartile (Q3), and the whiskers show the range of lower and higher adjacent values. Significant differences are indicated by * (paired t-test, Mann-Whitney $U$ method, $P<0.05$; not significant).

Finally, to disentangle if the observed effects were produced by an increase of the adaptation to repeated sounds or by an increase of the response to deviant sounds, we used the many-standard and cascade paradigms and calculated the iMM, the iPE and the iRS indices (Parras et al., 2017). This results revealed a significant increase of the iMM $(0.30 \pm 0.03$ vs. $0.17 \pm 0.04)$ and the $\operatorname{PPE}(0.17 \pm$ 0.04 vs. $0.05 \pm 0.04)$ for trained frequencies as compared to untrained frequencies (paired t-test, Mann-Whitney $U$ method, all $\mathrm{P}<0.05$ ), while there were no differences for the iRS $(0.15 \pm 0.05$ vs. $0.15 \pm 0.04$, n.s. $)$. Since the iMM is made of the sum of iPE and iRS, this result suggests that the larger values of iMM observed for trained frequencies are caused by an increase in the neuronal prediction error (Fig. 30), which enhances the responses to deviant tones. In conclusion, this data suggests that animals trained in an auditory discrimination task develop some sort of long-term plasticity that alters the representation of sounds, enhancing the neuronal response to behaviorally relevant sounds, while the levels of adaptation to behaviorally irrelevant sound remain unaltered. 
A

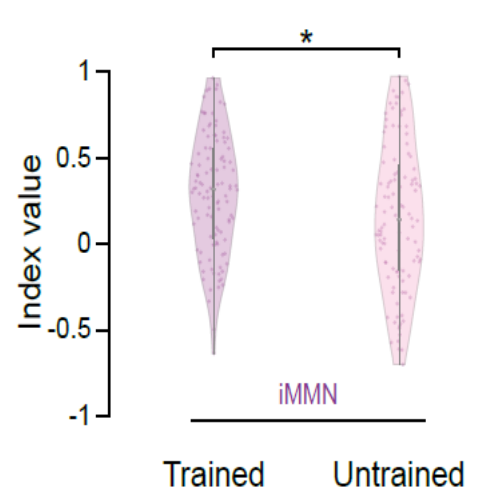

B

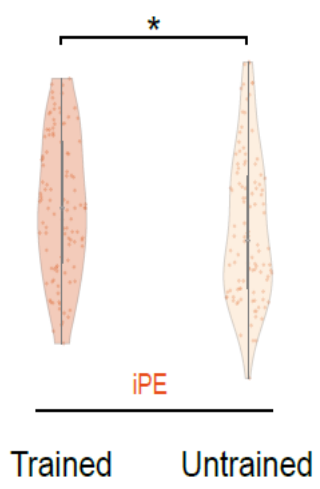

C

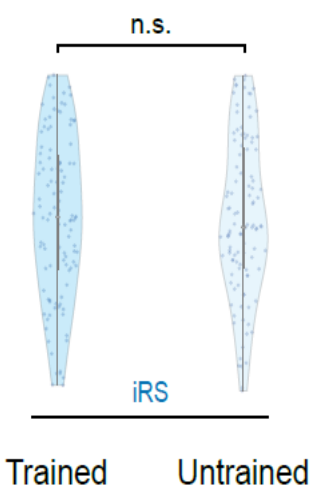

Figure 30: SSA elemental analysis after behavioral training. Violin plots showing indices of neuronal mismatch (iMM, in purple; $(\mathbf{A})$, prediction error (iPE, in orange; (B), and repetition suppression (iRS, in blue; (C) computed for all neuronal responses from trained animals to trained and untrained tone frequencies computed for neuronal responses to tone frequencies used in the behavioral training (Trained, left) and others new (Untrained, right) recorded from neuronal units of primary auditory cortex units $(53 ; 106$ pair of tones) in response to oddball paradigms from 14 trained animals distributed (averaged data). The thick grey bars expand from the first (Q1) to the third quartile (Q3), and the whiskers show the range of lower and higher adjacent values. Significant differences are indicated by ${ }^{*}$ (paired t-test, Mann-Whitney $U$ method, $\mathrm{P}<0.05$; no significant).

In summary, the principal conclusions of this work include: 1) rats can successfully discriminate the salience of deviant stimuli and 2) the training on the auditory discrimination task had effects on neuronal activity, increasing deviant detection, neuronal mismatch and prediction error responses. 


\section{DISCUSSION}

In this thesis, I have studied the electrophysiological characteristics and distribution of the neurons in lemniscal and non-lemniscal areas that generate deviance detection (fast spiking and regular spiking neurons). Next, I analyzed how ACh modulates deviance detection and error prediction levels in primary and secondary auditory cortical areas. Then, I studied the effect of behaviorally relevant sounds in saliency detection and prediction error response, in trained and not-trained rats to auditory behavioral paradigms. In the following, I will discuss the main results obtained and the functional significance and relevance of these findings.

Collectively, the results of my thesis suggest three major findings: 1) There are no specific neuronal types in AC for SSA and deviance detection; thus SSA is generated at the neuronal network; 2) my results have also revealed that ACh scales prediction error signals in the AC, likely gating these signals beyond the auditory cortex to frontal, higher cognitive regions; and 3 ) the present thesis also demonstrated that trained rats learn to detect a specific deviant tone from a regular pattern and furthermore, they can generalize this salience, at least, in the frequency domain.

\section{Inhibitory and excitatory auditory cortical neurons}

In Section I, we compared the deviance detection properties on putative excitatory and inhibitory single units recorded from the AC of anaesthetized rats. Putative inhibitory units had narrower spikes, increased firing rates and smaller spike amplitudes relative to putative excitatory units. While putative inhibitory 
units spread through all cortical layers, putative excitatory units were mainly confined to layers III-V. Both putative inhibitory and excitatory units showed deviance detection properties, and in both cases the prediction error component was more relevant than repetition suppression.

In the present study, we found a much larger percentage of putative inhibitory than excitatory neurons (74 vs $26 \%$, respectively). This apparent sampling bias does not seem to be related to the cell size (putative excitatory neurons show larger spike amplitudes and SNRs), although the custom-made, high impedance tungsten electrodes that we used probably allowed us to easily record from smaller sized neurons. It is also unlikely that this bias is due to any effect caused by the urethane anaesthesia we used. Previous studies have shown that urethane affects both excitatory and inhibitory currents in a non-selective manner (Hara and Harris, 2002), and it does not alter excitatory glutamate- nor GABAmediated synaptic transmission (Sceniak and Maclver, 2006).

After the seminal study from Mountcastle et al. (1969) that, in general terms proposed that inhibitory and excitatory cortical neurons could be distinguished by the shape of their spikes, other studies have found similar results, adding evidence in support of this type of classification (e.g., Hefti and Smith, 2000; Kawaguchi and Kondo, 2002; Moore and Wehr, 2013). This classification has proven very useful, since it adds relevant information about the function of the units (which is not available using conventional recordings) and can be applied directly to most extracellular recordings (without the need for additional, more complex techniques). It has been successfully used in multiple studies, although 
with some methodological variations, such as in the references used for measuring the spike widths (e.g., time from peak to trough: Atencio and Schreiner, 2008; half-width, i.e. full width at half-maximal amplitude: Chen et al., 2015; half-valley width: Insel and Barnes, 2015; valley to peak width and halfpeak width: Mendoza et al., 2016; Peyrache et al., 2012; width of "afterhyperpolarization": Andermann et al., 2004) or the aid of additional dimensions (firing rate, spike amplitude, peak asymmetry, etc.) to refine the classification. Previous studies using a spike width measurement methodology most similar to ours (Barthó et al., 2004; Insel and Barnes, 2015; Mendoza et al., 2016), found that the best spike width cut off for separating FS and RS would range between $\sim 0.25-0.4 \mathrm{~ms}$ (if not considering the other dimensions measured), which is analogous to the $0.35 \mathrm{~ms}$ cut off used here.

The distribution of fast- and regular spiking neurons

The general characteristics of our FS and RS units are in general agreement with previous reports. Apart from narrower spike widths, FS units showed increased firing rates and smaller spike amplitudes relative to RS units (Andermann et al., 2004; Atencio and Schreiner, 2008; Baeg et al., 2001; Bruno and Simons, 2002; Calabrese and Woolley, 2015; I. W. Chen et al., 2015; Hefti and Smith, 2000; Insel and Barnes, 2015; Mendoza et al., 2016; Moore and Wehr, 2013; Mountcastle et al., 1969; Peyrache et al., 2012). The distribution of FS and RS units across cortical layers differed in some degree from the actual locations of pyramidal neurons and interneurons reported using histological methods (Games and Winer, 1988). While putative inhibitory units were found across all cortical layers (with increased densities in layers II-V) as expected, putative excitatory 
units were found mainly in layers III-V, peaking at layer IV (Fig. 15B); however, according to histology, pyramidal neurons are very scarce in layer IV. Since the measurement of the recording depth in the present study was based on indirect methods (depth of the recording site as indicated on the microdrive), it is possible that the units estimated to be near the borders between layers could be actually located in adjacent layers. Indeed, layer IV spreads only about $100 \mu \mathrm{m}$, so it is not unlikely that a portion of neurons assigned to layer IV could easily be located in layers III or V.

Role of inhibition and deviant detection of fast- and regular spiking neurons The role of inhibition in the generation of deviance detection responses is still unclear. In subcortical nuclei, it has been shown that GABAergic inhibition has a modulatory effect, acting as a gain control mechanism that enhances and sharpens SSA (Duque et al., 2014; Pérez-González et al., 2012; Pérez-González and Malmierca, 2012). Many studies have found a dependence of MMN on Nmethyl-d-aspartate (NMDA) glutamate receptors, which would suggest a relevant role of excitatory cortical neurons in the generation of deviance detection, but we should not ignore the modulatory effect of inhibition on these responses (for a review, see Askew and Metherate, 2016). We found that both FS and RS units in the AC showed similarly high levels of deviance detection (measured as the Neuronal Mismatch Index, Fig. 16A-B). This finding conforms with previous studies that found that both excitatory and inhibitory (PV and SOM) neurons in the AC exhibit SSA (I. W. Chen et al., 2015; Natan et al., 2015). Using whole cell recordings, Chen et al. (I. W. Chen et al., 2015) found a long latency (mainly subthreshold) component in excitatory neurons, reflecting genuine deviance 
detection. Interestingly, Natan et al. (Natan et al., 2015) found that both types of interneurons contribute to increased SSA in excitatory neurons, but through different mechanisms: optogenetic suppression of SOM interneurons increased the response of excitatory neurons to the standard tones but not to the deviant tones, while suppression of PV interneurons produced an equal increase in the response of excitatory neurons to both standard and deviant tones. The potentially different roles of the various types of inhibitory cortical interneurons are paramount for defining the circuits involved in deviance detection; unfortunately, the methodology of the present study does not allow for the differentiation of subtypes of inhibitory neurons, but it clearly demonstrates that GABAergic neurons play indeed a key role in SSA and deviance detection generation.

The deviance detection properties of both FS and RS units were the result of a high contribution of the prediction error component and reduced repetition suppression. We found that both types of units show similar deviance detection capabilities, as shown by the distributions of iMM (Fig. 16A), and also in both cases the prediction error component (Fig. 16E) has a stronger weight on deviance detection than the repetition suppression component (Fig. 16C). But when looking individually at each field, FS units tended to show an average index values different from zero, which was not so common for RS units (Fig. 16B, D, F). At the same time, the distributions of RS and FS units for each field were similar, except for A1 (see below). However, we found some differences in the iMM across AC fields, but only for RS units (Fig. 16B). In this case, the putative excitatory units in A1 (a lemniscal field) showed smaller iMM values as compared 
to units in SRAF and PAF (both of them non-lemniscal fields). This is probably because RS units from SRAF and PAF tend to show larger iPE values than RS units in $A 1$, although this trend does not amount to a statistically significant difference. It is noteworthy that $A 1$ was the only $A C$ field where the iMM was different for RS and FS units (Fig. 16B); FS units showed a larger iMM than RS units, maybe due to larger iPE. As a lemniscal field, A1 tends to show reduced deviance detection properties compared to non-lemniscal fields (Parras et al., 2017b). While FS units from all AC fields have iMM statistically larger than zero, there is a clear distinction between lemniscal (A1, VAF and AAF) and nonlemniscal fields (SRAF, PAF) for RS units. In this group, only non-lemniscal fields have a Neuronal Mismatch Index significantly larger than zero. This would mean that while in lemniscal fields mostly FS units contribute to deviance detection, in non-lemniscal fields both FS and RS units could contribute to the larger deviance detection values found on those areas. But in any case, these findings suggest that SSA and deviance detection are generated at the neuronal network and that there is not specific neuronal types in AC for SSA.

Acetylcholine modulates SSA and prediction error response in auditory rat cortex In Section II, we recorded multiunit activity from AC neurons under an auditory oddball paradigm while performing microiontophoretic applications of $\mathrm{ACh}$ and corresponding muscarinic and nicotinic receptor antagonists to investigate the role of the cholinergic system in the processing of unexpected or surprising events. Furthermore, we employed cascade sequences to control for repetition suppression and distinctly assess cholinergic effects on prediction error relative to repetition suppression. Our results show that 1) ACh augments SSA by 
specifically increasing the response to the deviant tone (Fig. 31); 2) The opposite of this effect is found with the administration of a muscarinic antagonist, suggesting that this effect may be mediated by muscarinic ACh receptors; 3) ACh exerts a global and distributed effect on most AC neurons regardless of their field and layer location. Together, these findings reveal how ACh scales prediction error signals in the AC, likely gating these signals beyond the auditory cortex to frontal, higher cognitive regions.

ACh can exert its effects via mAChRs or nAChRs. We found that blocking muscarinic receptors with scopolamine led to a significant decrease in the neuronal firing rate in response to the deviant tone. In contrast, the nAChR blocker, mecamylamine, had no effect. Interestingly, the fact that blocking mAChRs only reduced the responses to deviant tones (without the application of $\mathrm{ACh}$ ) indicates that there is intrinsic release of Ach, suggesting that such a release may be specifically linked to the occurrence of the deviant tones (Fig. 31). These results are similar to those from a study in the auditory midbrain (Ayala and Malmierca, 2015), where scopolamine, but not mecamylamine, affected SSA in the inferior colliculus (IC). However, in the IC, ACh selectively increased the response to the repetitive standard stimulus (Ayala and Malmierca, 2015), while we show here that $\mathrm{ACh}$ in the $\mathrm{AC}$ only increases the responses to the unexpected deviant (Fig. 31). This divergent effect of ACh on subcortical and cortical SSA may be related to the different origin of $\mathrm{ACh}$ and/or the unique organization of neuronal circuitries in IC and AC (Fig. 31). While the sources of ACh to IC emerge from the pedunculopontine and laterodorsal tegmental regions in the brainstem (Motts and Schofield, 2009), those to AC originate in the basal forebrain (Chavez 
and Zaborszky, 2017). The differential distribution of mAChRs and nAChRs in AC and IC is also noteworthy. While nAChRs are most abundant in supragranular layers of the cortex, mAChRs can be found throughout all cortical layer (Colangelo et al., 2019; Edeline, 2012). This is consistent with our finding that neurons whose SSA was affected by ACh were found similarly in all cortical layers, supporting the idea that ACh effect on SSA is mostly mediated by mAChRs and not by nAChRs. Moreover, this mAChRs influence on SSA would increase the cortical coding capacity of the deviant stimulus through an enhancement of postsynaptic excitability (Goard and Dan, 2009; Metherate, 2011).

Overall, the main effect of $\mathrm{ACh}$ was an increase in neuronal firing. Strong cholinergic activation, originating in the basal forebrain, is associated with active arousal states (Batista-Brito et al., 2018; Hasselmo, 1999). Likewise, cortical inhibitory interneurons play a crucial role in sensory processing and regulate what is represented and transmitted throughout cortical circuits. In this context, it is interesting to mention that recent studies have revealed and characterized the details of organization of canonical cortical circuits that underlie the translation of neuromodulatory signals into changes in sensory processing (reviewed in (Batista-Brito et al., 2018; and Picciotto et al., 2012) ).Thus, ACh can directly affect the responses of pyramidal neurons in $A C$, boosting response gain (Batista-Brito et al., 2018; Noudoost and Moore, 2011; Wood et al., 2017).

ACh can also indirectly disinhibit pyramidal neurons through the activation of vasoactive intestinal peptide (VIP) interneurons, which express cholinergic 
receptors (Tremblay et al., 2016); VIP interneurons strongly inhibit somatostatin (SST) interneurons, that in turn inhibit pyramidal neurons. Yet another possibility is that ACh can directly activate SST interneurons, which inhibit parvalbumin (PV) interneurons (Xu et al., 2013), producing a similar disinhibitory effect on pyramidal neurons. In addition, it has been shown that optogenetic photosuppression of PVmediated inhibition in AC leads to a nonspecific increase of neuronal responses, enhancing equally the responses to deviant and standard tones, while similar optogenetic photosuppression of SST-mediated inhibition selectively reduces excitatory responses to frequent tones (Natan et al., 2015). Moreover, longlasting habituation involves a selective increase in SST-mediated inhibition (Kato et al., 2015). Thus, there are multiple opportunities by which ACh can modulate SSA, acting through several mechanisms and microcircuits involving VIP, SST and PV inhibitory interneurons. Indeed, in the previous Section, we have demonstrated that FS neurons, i.e., putative inhibitory neurons show robust SSA throughout the entire AC. Future studies on the effect on noradrenergic input from the locus coeruleus to AC should be also considered as they also play a role in active arousal states (Batista-Brito et al., 2018; Hasselmo, 1999). Regardless of which specific circuits are involved, here we show that cholinergic modulation produces an increase in sensory gain in $A C$, by increasing the firing rate in response to the deviant tone ACh (Muñoz et al., 2017; Muñoz and Rudy, 2014). This, in turn, may increase the impact of top-down inputs, promoting favorable conditions for changes in cortical plasticity such as SSA (Batista-Brito et al., 2018). 


\section{ACh, SSA and predictive coding framework}

According to the predictive coding theory, cortical areas send prediction information to lower hierarchical areas to suppress expected neural activity and thus help anticipate sensory events. Conversely, those lower areas send error information to higher hierarchical centers when event predictions fail. If a stimulus is unexpected, the difference between expected and received inputs yields large prediction errors, while if a stimulus is constantly repeated, it yields a decrease in neural responsivity a phenomenon referred to as repetition suppression (Auksztulewicz and Friston, 2016). Thus, SSA can be explained under the predictive coding framework (G. V Carbajal and Malmierca, 2018; Parras et al., 2017b). However, predictions and prediction errors may also be modulated in a context-dependent manner, meaning that responses can be modulated and given precedence depending on the context in which the stimulus is perceived (Keller and Mrsic-Flogel, 2018). The source of such a modulating or gating signal may arise from neuromodulatory inputs (Fu et al., 2014; Moran et al., 2013; Pinto et al., 2013; Polack et al., 2013; Thiele and Bellgrove, 2018) that can not only gate plasticity (Kilgard and Merzenich, 1998; Martins and Froemke, 2015; Weinberger, 2004) but also change the balance of top-down versus bottom-up influence (Yu and Dayan, 2005). Within the predictive coding framework, classical neuromodulators such as ACh are often thought to increase the precision of prediction error signaling (Auksztulewicz et al., 2018; Auksztulewicz and Friston, 2016; Feldman and Friston, 2010; Moran et al., 2013) and this is precisely what our results confirm empirically at the neuronal level. Using computational neuronal modeling and EEG recordings under oddball paradigms in subjects manipulated pharmacologically with galantamine (a competitive inhibitor of 
acetylcholinesterase), Moran et al. (2013) have shown that ACh enhances the precision of bottom-up synaptic transmission in cortical hierarchies by optimizing the gain of supragranular pyramidal neurons, amounting to an increase in the precision of prediction error signalling. Thus, our results and those from Moran and colleagues (2013) suggest that ACh mediates the representation of precision and acts to facilitate the bottom-up signaling of sensory information (through precise prediction errors). In this context, Yu and Dayan proposed that ACh levels reflect the "expected uncertainty" (when there is less confidence in the prediction) associated with top-down information and modulate the interaction between topdown and bottom-up processing in determining the appropriate neural representations for inputs (Auksztulewicz et al., 2017; Yu and Dayan, 2005, 2002) Recent studies have emphasized the relationship between global networks in neural oscillations and predictive coding during stimulus processing (Chao et al., 2018; Sikkens et al., 2019). Indeed, a large body of evidence, including studies in both primates and humans, have proven that feedforward, and feedback communication between cortical areas is transmitted by gamma and alpha/betaband oscillations, respectively (Bastos et al., 2012b; Dürschmid et al., 2016; Fontolan et al., 2014; Michalareas et al., 2016; Recasens et al., 2018; Sedley et al., 2016).

Gamma oscillations have been found recently to be dependent on SST neuronal activity (Veit et al., 2017) and several human studies using electrocorticography and magnetoencephalography have demonstrated the correlation between prediction errors and the magnitude of gamma-band oscillations in audition (Dürschmid et al., 2016; Edwards et al., 2005; Todorovic et al., 2011) and in other 
sensory systems (Auksztulewicz et al., 2017). ACh activation maintains a high postsynaptic gain and facilitates a spectral shift of neural oscillations toward higher (gamma-band) frequencies (Liljenstrom and Hasselmo, 1995; Moran et al., 2013).Our finding that $A C h$ gates neuronal prediction errors in the $A C$ further supports the notion that activity in the gamma frequency band maybe is linked to error signals originating from sensory areas like AC (Fig. 31), which convey prediction errors towards hierarchically higher brain areas.

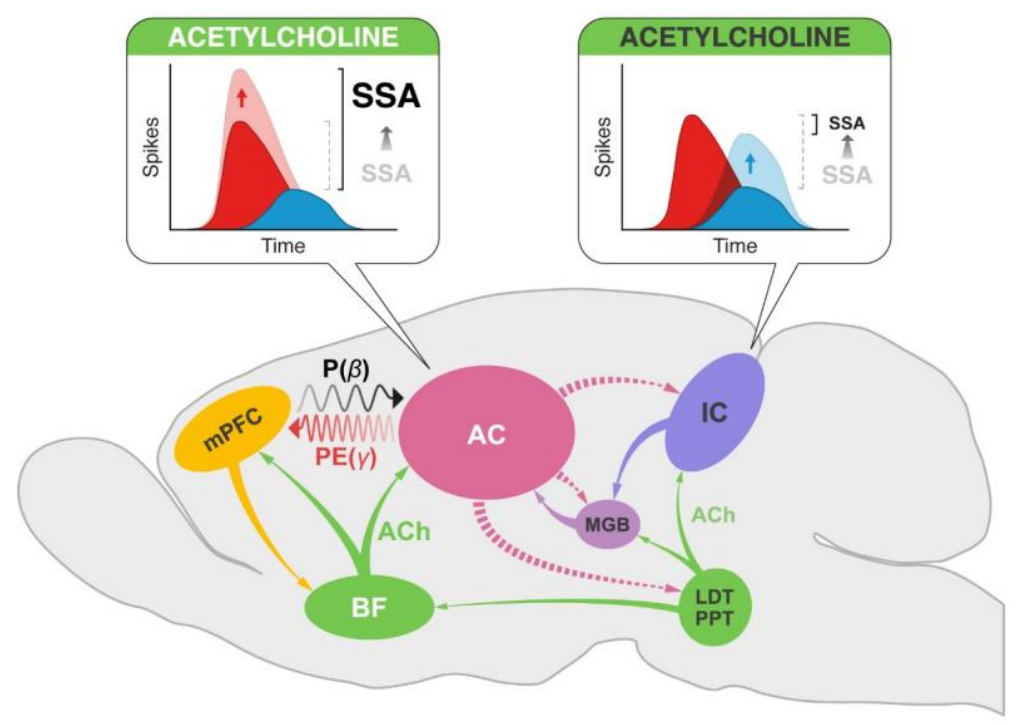

Figure 31: Effect of ACh on SSA in auditory midbrain and cortex. $A C h$ increases SSA in AC scaling the firing rate in response to the 'deviants', while it shows an opposite effect in the IC, as ACh decreases SSA by scaling the responses to the 'standard' tones (Ayala and Malmierca, 2015). These divergent effects may be explained by the different connectivity of both auditory areas. While the AC and medial prefrontal cortex (mPFC) receive their cholinergic input from the basal forebrain (BF), the IC is innervated by the pedunculopontine (PPT) and laterodorsal tegmental (LDT) regions in the brainstem. This intersection of auditory and cholinergic nuclei creates an intricate network involving ascending and descending projections that ultimately modulate the processing of auditory deviance detection, such that $\mathrm{ACh}$ increases the precision of prediction error signaling in $A C$, gating prediction errors $(P E)$ to hierarchically higher processing levels (mPFC). 


\section{Deviance detection and cholinergic modulation in humans}

The cholinergic modulation of deviant detection has also been studied in humans. Klinkenberg and colleagues report that the use of rivastigmine (cholinesterase inhibitor) increases the P3a amplitude, a component related to novelty processing in auditory event-related potentials (Klinkenberg et al., 2013), while Caldenhove et al. reports that the use of biperiden (muscarinic M1-type antagonist) reduces the P3a amplitude (Caldenhove et al., 2017). Recently, the use of deep brain stimulation, a novel technique in which electrodes are implanted in the brain can then be used to deliver electrical impulses into a specific area, has been explored like a therapy for the treatment in patients with dementias, like Alzheimer disease (Kumbhare et al., 2018). Alzheimer disease is characterized by a significant decrease of the innervation of the cortex as a result of the Nucleus Basal of Meynert, degeneration, which is well known to be the principal cholinergic source in the basal forebrain to the cortex (Mesulam, 2013). Interestingly, Kuhn et al. reported that the treatment using deep brain stimulation of the NBM in 4 of 6 patients suffering of Alzheimer's disease showed a stable or even improved cognitive functioning after 12 months (J. Kuhn et al., 2015; Jens Kuhn et al., 2015). Furthermore, Dürschmid et al. suggested that deep brain stimulation of the nucleus basal of Meynert improves sensory gating of familiar auditory information into sensory memory (Dürschmid et al., 2016).

\section{Behavioral discrimination task in rat auditory cortex}

In Section III we have trained rats in a tone discrimination task to detect deviant sounds under the oddball paradigm. We have shown that rats were not only detecting a specific deviant tone but generalized and responded to any violation 
in the frequency domain stablished by a standard repeating tone. Such behavioral training had several long-term effects when evaluating neuronal responses in A1. The SSA response was larger for trained animals than for naïve animals. Moreover, in trained rats, frequencies used during behavioral training showed larger levels of SSA than frequencies not used during training and the cascade paradigm showed that such modification of the representation of the sounds in trained animals was caused by a specific enhancement of the response to deviant tones rather than a decrease in response to standard tones. These results suggest some type of long-term memory trace that can shape the level of adaptation of the neurons according to its behavioral relevance.

\section{Comparisons to previous behavioral studies}

The current study was designed to stablish a correlate between the widely use oddball paradigm in electrophysiological works and a behavior discrimination task. All animals performed the auditory discrimination task, detecting the deviant tone with a performance larger than $80 \%$. Small discrimination differences were observed between the different groups depending on the frequencies used during training, but since all pairs of frequencies chosen are close in the auditory threshold of this rat strain (Popelar et al., 2006), we attributed differences between trained groups just to the animal skills. First evidence of a behavior frequency discrimination task with awake animals was presented by Recanzone et al. (1993), where they showed that monkeys trained under an oddball paradigm were able to detect the deviant tone (Recanzone et al., 1993). Several studies have demonstrated that cats (Hubel et al., 1959), monkeys (Gottlieb et al., 1989), guinea pigs (Bakin and Weinberger, 1990), ferrets (Fritz et al., 2005, 2003) and 
rodents (Ceballo et al., 2019; Jaramillo and Zador, 2011; Znamenskiy and Zador, 2013) can perform a sound discrimination task under a paradigm similar to an oddball paradigm.

\section{Behavioral relevance modifies neuronal adaptation in A1}

It is well known that associative learning can modify the representation of auditory information in Al (Froemke et al., 2007; Schreiner and Polley, 2014; Weinberger, 2007). Most of the neurons recorded in A1 for this study showed SSA, responding with a firing rate significantly larger to the same tone as deviant than as standard, for both untrained and trained frequencies in trained animals as well for naïve animals. This data is in accordance with a great bulk of work showing SSA as an ubiquitous phenomenon in the auditory pathway (Antunes et al., 2010; Farley et al., 2010; Manuel S. Malmierca et al., 2009; Nieto-Diego and Malmierca, 2016; Pérez-González et al., 2005; Ulanovsky et al., 2003).

To understand if training on the auditory discrimination task affected the level of SSA, we test if there was any difference in CSI around neuronal best frequency between a pair of frequencies used and not used in training. We observed that the CSI values obtained in trained animals for pairs of frequencies used in the behavior protocol were significantly larger than the CSI values obtained for frequencies not used in the behavioral task. Nevertheless, increased values of SSA may be either related with an increase of adaptation to repeated sounds or with an increased response to deviant sounds. To disambiguate that, we recorded neuronal responses to control sequences (MSC-CASCADE paradigms) and compared them to the oddball paradigm to calculate the iMMN, iPE and iRS 
indices (Parras et al., 2017b; Valdés-Baizabal et al., 2020b). The results revealed the larger values of iMM observed for trained frequencies are caused by a specific increase in the neuronal firing rate to the deviant tone rather than a decrease in firing rate to the repeated standard tone.

According to Feldman \& Friston (2010) the prediction error signal is weighted by its precision, more precise iPE elicits larger responses. This is supposed to be the mechanism through with attention operates. That is, attention modulates the iPE signal adjusting its gain, and paying attention to a stimulus means to increase the precision. Thus, more attention $=$ increased precision $=$ increased gain $=$ larger PE. Moreover, there is currently a lot of debate regarding the definitions of attention and prediction, since these two concepts have been quite mixed in previous research, and a proposal which is currently well accepted is to conceptualize attention exclusively as task-relevance (Summerfield et al., 2008). Thus, after training, the sounds have become task-relevant, which means that the animals pay more attention to them, and therefore the PE should be larger. Interestingly, ACh potentiate attention and plays a major role in synaptic plasticity (Bentley et al., 2011; Hasselmo and Giocomo, 2006; Newman et al., 2012) and our Study II using the microiontophretic technique demonstrate that ACh plays a unique role in augmenting SSA, and more precisely PE in rat $A C$ increasing the precision of prediction error signaling. The current data support this notion and goes beyond in that it shows a behavioral correlate of SSA and PE in the awake, behaving rat. 
Another similar work was recently being published (Yaron et al., 2020). They also conditioned awake freely moving rats to perform an auditory discrimination task, but the used an aversive stimulus. While we observed an increase in the neuronal response to conditioned stimuli (deviant) foody rewarded, and a subsequent increment of SSA index. In contrast to our results, Yaron and colleagues (2020) have reported that the contrast between standard and deviant responses remained the same or even decreased for conditioned stimuli but increased for unconditioned stimuli. Both studies (Yaron et al., 2020, and present results) show divergent examples of SSA being shaped by experience but with a similar functional significance. Fear conditioning might have a different behavior role, and the reduction of the neuronal response to the aversive conditioned stimuli could be beneficial. In the same vein, an increase in response to a rewarded stimulus would be advantageous, too.

\section{Long-term plasticity mechanisms to behavioral relevant sounds}

It has been described that SSA is a basic adaptation mechanism which involve synaptic depression or intrinsic cellular mechanisms (Eytan et al., 2003), acting differentially at diverse parts of related neurons to optimize the postsynaptic sensitivity of neurons showing SSA (Carbajal and Malmierca, 2018; Garrido et al., 2009a). To compute the novelty, a network is required which compares the neural responses to the current stimulus with previous responses based on an integration of information about stimuli characteristics modulations (Gutfreund, 2012). The memory trace of adaptation of neural responses has been mostly studied in short-term events ( $2 s$ - tens of minutes), but behavior times requires a time scale adjustment (Dutta and Gutfreund, 2014; Gutfreund, 2012). Our work 
contributes to study SSA in a larger scale, describing neuronal adaptation effects 3 days after finishing the behavior task and under anesthesia. This data suggests that animals trained in an auditory discrimination task develop some sort of longterm plasticity that alters the representation of sounds, enhancing the neuronal response to behaviorally relevant sounds, while the levels of adaptation to behaviorally irrelevant sound remain unaltered. 


\section{Conclusions}

1) The analysis of spike shape from extracellular recordings in the anesthetized rat $A C$ can be used to classify the units as putative excitatory or inhibitory.

2) Both putative excitatory and inhibitory neurons in the rat AC show similar levels of deviance detection. These results suggest the involvement of both types of neurons in the circuits that generate deviance detection and that SSA is generated at the network level.

3) Local application of ACh increases neural excitability in the auditory cortex of the rat

4) Cholinergic modulation scales SSA in auditory cortex, and more specifically, ACh increases the levels of prediction error setting the gain of prediction error units, optimizing the signaling of precision in the brain.

5) Trained rats in an auditory discrimination task can successfully discriminate the salience of deviant, low probability sounds embedded in a sequence of repeating, high probability sounds.

6) Trained rats in an auditory discrimination task develop some sort of longterm plasticity that alters the representation of sounds, enhancing the neuronal response to behaviorally relevant sounds. 


\section{REFERENCES}

Andermann, M.L., Ritt, J., Neimark, M.A., Moore, C.I., 2004. Neural correlates of vibrissa resonance: Band-pass and somatotopic representation of highfrequency stimuli. Neuron 42, 451-463. https://doi.org/10.1016/S08966273(04)00198-9

Anderson, L.A., Malmierca, M.S., 2013. The effect of auditory cortex deactivation on stimulus-specific adaptation in the inferior colliculus of the rat. Eur. J. Neurosci. https://doi.org/10.1111/ejn.12018

Antunes, F.M., Malmierca, M.S., 2011. Effect of auditory cortex deactivation on stimulus-specific adaptation in the medial geniculate body. J. Neurosci. https://doi.org/10.1523/JNEUROSCI.1915-11.2011

Antunes, F.M., Nelken, I., Covey, E., Malmierca, M.S., 2010. Stimulus-specific adaptation in the auditory thalamus of the anesthetized rat. PLoS One. https://doi.org/10.1371/journal.pone.0014071

Askew, C.E., Metherate, R., 2016. Synaptic interactions and inhibitory regulation in auditory cortex. Biol. Psychol. 116, 4-9. https://doi.org/10.1016/j.biopsycho.2015.11.001

Atencio, C.A., Schreiner, C.E., 2008. Spectrotemporal processing differences between auditory cortical fast-spiking and regular-spiking neurons. J. Neurosci. 28, 3897-3910. https://doi.org/10.1523/JNEUROSCI.536607.2008

Auksztulewicz, R., Friston, K., 2016. Repetition suppression and its contextual determinants in predictive coding. Cortex. https://doi.org/10.1016/j.cortex.2015.11.024

Auksztulewicz, R., Friston, K.J., Nobre, A.C., 2017. Task relevance modulates 
the behavioural and neural effects of sensory predictions. PLoS Biol. 15, e2003143. https://doi.org/10.1371/journal.pbio.2003143

Auksztulewicz, R., Schwiedrzik, C.M., Thesen, T., Doyle, W., Devinsky, O., Nobre, A.C., Schroeder, C.E., Friston, K.J., Melloni, L., 2018. Not all predictions are equal: "what" and "when" predictions modulate activity in auditory cortex through different mechanisms. J. Neurosci. 38, 8680-8693. https://doi.org/10.1523/JNEUROSCI.0369-18.2018

Ayala, Y.A., Malmierca, M.S., 2015. Cholinergic modulation of stimulus-specific adaptation in the inferior colliculus. J. Neurosci. 35, 12261-12272. https://doi.org/10.1523/JNEUROSCI.0909-15.2015

Ayala, Y.A., Pérez-González, D., Duque, D., Nelken, I., Malmierca, M.S., 2012. Frequency discrimination and stimulus deviance in the inferior colliculus and cochlear nucleus. Front. Neural Circuits.

https://doi.org/10.3389/fncir.2012.00119

Baeg, E.H., Kim, B.K., Jang, J., Kim, H.T., Mook-Jung, I., Jung, M.W., 2001. Fast Spiking and Regular Spiking Neural Correlates of Fear Conditioning in the Medial Prefrontal Cortex of the Rat. Cereb. Cortex 11, 441-451. https://doi.org/10.1093/cercor/11.5.441

Bakin, J.S., Weinberger, N.M., 1990. Classical conditioning induces CS-specific receptive field plasticity in the auditory cortex of the guinea pig. Brain Res. 536, 271-286. https://doi.org/10.1016/0006-8993(90)90035-A

Barthó, P., Hirase, H., Monconduit, L., Zugaro, M., Harris, K.D., Buzsáki, G., 2004. Characterization of neocortical principal cells and interneurons by network interactions and extracellular features. J. Neurophysiol. 92, 600608. https://doi.org/10.1152/jn.01170.2003 
Bastos, A.M., Usrey, W.M., Adams, R.A., Mangun, G.R., Fries, P., Friston, K.J., 2012a. Canonical Microcircuits for Predictive Coding. Neuron. https://doi.org/10.1016/j.neuron.2012.10.038

Bastos, A.M., Usrey, W.M., Adams, R.A., Mangun, G.R., Fries, P., Friston, K.J., 2012b. Canonical Microcircuits for Predictive Coding. Neuron. https://doi.org/10.1016/j.neuron.2012.10.038

Batista-Brito, R., Zagha, E., Ratliff, J.M., Vinck, M., 2018. Modulation of cortical circuits by top-down processing and arousal state in health and disease. Curr. Opin. Neurobiol. https://doi.org/10.1016/j.conb.2018.06.008

Bendixen, A., 2014. Predictability effects in auditory scene analysis: A review. Front. Neurosci. https://doi.org/10.3389/fnins.2014.00060

Bentley, P., Driver, J., Dolan, R.J., 2011. Cholinergic modulation of cognition: Insights from human pharmacological functional neuroimaging. Prog. Neurobiol. https://doi.org/10.1016/j.pneurobio.2011.06.002

Bruno, R.M., Simons, D.J., 2002. Feedforward mechanisms of excitatory and inhibitory cortical receptive fields. J. Neurosci. 22, 10966-10975.

Calabrese, A., Woolley, S.M.N., 2015. Coding principles of the canonical cortical microcircuit in the avian brain. Proc. Natl. Acad. Sci. 112, 35173522. https://doi.org/10.1073/pnas.1408545112

Caldenhove, S., Borghans, L.G.J.M., Blokland, A., Sambeth, A., 2017. Role of acetylcholine and serotonin in novelty processing using an oddball paradigm. Behav. Brain Res. https://doi.org/10.1016/j.bbr.2017.05.031

Calford, M.B., Aitkin, L.M., 1983. Ascending projections to the medial geniculate body of the cat: Evidence for multiple, parallel auditory pathways through thalamus. J. Neurosci. 3, 2365-2380. https://doi.org/10.1523/jneurosci.03- 
$11-02365.1983$

Carbajal, Guillermo Varela, Malmierca, M.S., 2018. The unique role of the nonlemniscal pathway on stimulus-specific adaptation (SSA) in the auditory system, in: Proceedings of the International Symposium on Auditory and Audiological Research.

Carbajal, Guillermo V., Malmierca, M.S., 2018. The Neuronal Basis of Predictive Coding Along the Auditory Pathway: From the Subcortical Roots to Cortical Deviance Detection. Trends Hear. https://doi.org/10.1177/2331216518784822

Carbajal, G. V, Malmierca, M.S., 2018. The Neuronal Basis of Predictive Coding Along the Auditory Pathway: From the Subcortical Roots to Cortical Deviance Detection Introduction: SSA and MMN, Two Faces of Deviance Detection. journals.sagepub.com 22. https://doi.org/10.1177/2331216518784822

Casado-Román, L., Pérez-González, D., Malmierca, M.S., 2019. Prediction errors explain mismatch signals of neurons in the medial prefrontal cortex. bioRxiv 778928. https://doi.org/10.1101/778928

Ceballo, S., Piwkowska, Z., Bourg, J., Daret, A., Bathellier, B., 2019. Targeted Cortical Manipulation of Auditory Perception. Neuron 104, 1168-1179.e5. https://doi.org/10.1016/j.neuron.2019.09.043

Chao, Z.C., Takaura, K., Wang, L., Fujii, N., Dehaene, S., 2018. Large-Scale Cortical Networks for Hierarchical Prediction and Prediction Error in the Primate Brain. Neuron 100, 1252-1266.e3. https://doi.org/10.1016/j.neuron.2018.10.004

Chavez, C., Zaborszky, L., 2017. Basal Forebrain Cholinergic-Auditory Cortical 
Network: Primary Versus Nonprimary Auditory Cortical Areas. Cereb.

Cortex 27, 2335-2347. https://doi.org/10.1093/cercor/bhw091

Chen, I.-W., Helmchen, F., Lütcke, H., 2015. Specific Early and Late Oddball-

Evoked Responses in Excitatory and Inhibitory Neurons of Mouse Auditory

Cortex. Soc Neurosci. https://doi.org/10.1523/JNEUROSCI.2240-15.2015

Chen, I.W., Helmchen, F., Lütcke, H., 2015. Specific early and late oddballevoked responses in excitatory and inhibitory neurons of mouse auditory cortex. J. Neurosci. 35, 12560-12573.

https://doi.org/10.1523/JNEUROSCI.2240-15.2015

Colangelo, C., Shichkova, P., Keller, D., Markram, H., Ramaswamy, S., 2019.

Cellular, synaptic and network effects of acetylcholine in the neocortex.

Front. Neural Circuits. https://doi.org/10.3389/fncir.2019.00024

Coomes, D.L., Schofield, B.R., 2004. Separate projections from the inferior colliculus to the cochlear nucleus and thalamus in guinea pigs. Hear. Res. https://doi.org/10.1016/j.heares.2004.01.009

Dalley, J.W., Cardinal, R.N., Robbins, T.W., 2004. Prefrontal executive and cognitive functions in rodents: Neural and neurochemical substrates, in: Neuroscience and Biobehavioral Reviews. pp. 771-784. https://doi.org/10.1016/j.neubiorev.2004.09.006

Disney, A.A., Aoki, C., Hawken, M.J., 2007. Gain Modulation by Nicotine in Macaque V1. Neuron. https://doi.org/10.1016/j.neuron.2007.09.034

Duque, D., Malmierca, M.S., Caspary, D.M., 2014. Modulation of stimulusspecific adaptation by GABAA receptor activation or blockade in the medial geniculate body of the anaesthetized rat. J. Physiol. 592, 729-743. https://doi.org/10.1113/jphysiol.2013.261941 
Duque, D., Pérez-González, D., Ayala, Y.A., Palmer, A.R., Malmierca, M.S., 2012. Topographic distribution, frequency, and intensity dependence of stimulus-specific adaptation in the inferior colliculus of the rat. J. Neurosci. https://doi.org/10.1523/JNEUROSCI.3190-12.2012

Dürschmid, S., Edwards, E., Reichert, C., Dewar, C., Hinrichs, H., Heinze, H.J., Kirsch, H.E., Dalal, S.S., Deouell, L.Y., Knight, R.T., 2016. Hierarchy of prediction errors for auditory events in human temporal and frontal cortex. Proc. Natl. Acad. Sci. U. S. A. 113, 6755-6760. https://doi.org/10.1073/pnas.1525030113

Dutta, A., Gutfreund, Y., 2014. Saliency mapping in the optic tectum and its relationship to habituation. Front. Integr. Neurosci. https://doi.org/10.3389/fnint.2014.00001

Edeline, J.M., 2012. Beyond traditional approaches to understand the functional role of neuromodulators in sensory cortices. Front. Behav. Neurosci. https://doi.org/10.3389/fnbeh.2012.00045

Edwards, E., Soltani, M., Deouell, L.Y., Berger, M.S., Knight, R.T., 2005. High gamma activity in response to deviant auditory stimuli recorded directly from human cortex. J. Neurophysiol. 94, 4269-4280. https://doi.org/10.1152/jn.00324.2005

Eggermann, E., Kremer, Y., Crochet, S., Petersen, C.C.H., 2014. Cholinergic Signals in Mouse Barrel Cortex during Active Whisker Sensing. Cell Rep. https://doi.org/10.1016/j.celrep.2014.11.005

Eytan, D., Brenner, N., Marom, S., 2003. Selective Adaptation in Networks of Cortical Neurons. J. Neurosci. 23, 9349-9356. https://doi.org/10.1523/jneurosci.23-28-09349.2003 
Farley, B.J., Quirk, M.C., Doherty, J.J., Christian, E.P., 2010. Stimulus-specific adaptation in auditory cortex is an NMDA-independent process distinct from the sensory novelty encoded by the mismatch negativity. J. Neurosci. 30, 16475-16484. https://doi.org/10.1523/JNEUROSCI.2793-10.2010

Feldman, H., Friston, K.J., 2010. Attention, uncertainty, and free-energy. Front. Hum. Neurosci. 4. https://doi.org/10.3389/fnhum.2010.00215

Fontolan, L., Morillon, B., Liegeois-Chauvel, C., Giraud, A.L., 2014. The contribution of frequency-specific activity to hierarchical information processing in the human auditory cortex. Nat. Commun. 5, 1-10. https://doi.org/10.1038/ncomms5694

Franklin, N.T., Frank, M.J., 2015. A cholinergic feedback circuit to regulate striatal population uncertainty and optimize reinforcement learning. Elife 4. https://doi.org/10.7554/eLife.12029

Friston, K., 2010. The free-energy principle: A unified brain theory? Nat. Rev. Neurosci. https://doi.org/10.1038/nrn2787

Friston, K., 2008. Hierarchical Models in the Brain. Cit. Frist. K 4, 1000211. https://doi.org/10.1371/journal.pcbi.1000211

Friston, K., 2005. A theory of cortical responses. royalsocietypublishing.org 360, 815-836. https://doi.org/10.1098/rstb.2005.1622

Friston, K., Kiebel, S., 2009. Cortical circuits for perceptual inference. Neural Networks 22, 1093-1104. https://doi.org/10.1016/j.neunet.2009.07.023 Fritz, J., Elhilali, M., Shamma, S., 2005. Active listening: Task-dependent plasticity of spectrotemporal receptive fields in primary auditory cortex. Hear. Res. 206, 159-176. https://doi.org/10.1016/j.heares.2005.01.015 Fritz, J., Shamma, S., Elhilali, M., Klein, D., 2003. Rapid task-related plasticity 
of spectrotemporal receptive fields in primary auditory cortex. Nat.

Neurosci. 6, 1216-1223. https://doi.org/10.1038/nn1141

Froemke, R.C., Merzenich, M.M., Schreiner, C.E., 2007. A synaptic memory trace for cortical receptive field plasticity. Nature 450, 425-429. https://doi.org/10.1038/nature06289

Fu, Y., Tucciarone, J.M., Espinosa, J.S., Sheng, N., Darcy, D.P., Nicoll, R.A., Huang, Z.J., Stryker, M.P., 2014. A cortical circuit for gain control by behavioral state. Cell 156, 1139-1152. https://doi.org/10.1016/j.cell.2014.01.050

Games, K.D., Winer, J.A., 1988. Layer V in rat auditory cortex: Projections to the inferior colliculus and contralateral cortex. Hear. Res. https://doi.org/10.1016/0378-5955(88)90047-0

Garrido, M.I., Kilner, J.M., Kiebel, S.J., Friston, K.J., 2009a. Dynamic causal modeling of the response to frequency deviants. J. Neurophysiol. 101, 2620-2631. https://doi.org/10.1152/jn.90291.2008

Garrido, M.I., Kilner, J.M., Stephan, K.E., Friston, K.J., 2009b. The mismatch negativity: A review of underlying mechanisms. Clin. Neurophysiol. https://doi.org/10.1016/j.clinph.2008.11.029

Ghazanfar, A.A., Schroeder, C.E., 2006. Is neocortex essentially multisensory? Trends Cogn. Sci. https://doi.org/10.1016/j.tics.2006.04.008

Goard, M., Dan, Y., 2009. Basal forebrain activation enhances cortical coding of natural scenes. Nat. Neurosci. 12, 1444-1449. https://doi.org/10.1038/nn.2402

Gottlieb, Y., Vaadia, E., Abeles, M., 1989. Single unit activity in the auditory cortex of a monkey performing a short term memory task. Exp. Brain Res. 
74, 139-148. https://doi.org/10.1007/BF00248287

Guillermo V. Carbajal and Manuel S. Malmierca, 2020. Novelty Processing in the Auditory System: Detection, Adaptation or Expectation?, in: Grothe Benedick, F.B. (Ed.), The Senses: A Comprehensive Reference, 2nd Edition. Elsevier.

Gutfreund, Y., 2012. Stimulus-specific adaptation, habituation and change detection in the gaze control system. Biol. Cybern. 106, 657-668. https://doi.org/10.1007/s00422-012-0497-3

Hackett, T.A., 2015. Anatomic organization of the auditory cortex, in: Handbook of Clinical Neurology. https://doi.org/10.1016/B978-0-444-62630-1.00002-0 Hara, K., Harris, R.A., 2002. The anesthetic mechanism of urethane: the effects on neurotransmitter-gated ion channels. Anesth Analg 94, 313-318.

Hardy, C.J.D., Yong, K.X.X., Goll, J.C., Crutch, S.J., Warren, J.D., 2020. Impairments of auditory scene analysis in posterior cortical atrophy. Brain 143, 2689-2695. https://doi.org/10.1093/brain/awaa221

Harms, L., Parras, G.G., Michie, P.T., Malmierca, M.S., 2020. The Role of Glutamate Neurotransmission in Mismatch Negativity (MMN), A Measure of Auditory Synaptic Plasticity and Change-detection. Neuroscience. https://doi.org/10.1016/j.neuroscience.2020.01.046

Hasselmo, M.E., 1999. Neuromodulation: Acetylcholine and memory consolidation. Trends Cogn. Sci. https://doi.org/10.1016/S13646613(99)01365-0

Hasselmo, M.E., Giocomo, L.M., 2006. Cholinergic modulation of cortical function, in: Journal of Molecular Neuroscience. pp. 133-135. https://doi.org/10.1385/JMN:30:1:133 
Hasselmo, M.E., Sarter, M., 2011. Modes and models of forebrain cholinergic neuromodulation of cognition. Neuropsychopharmacology. https://doi.org/10.1038/npp.2010.104

Hefti, B.J., Smith, P.H., 2000. Anatomy, physiology, and synaptic responses of rat layer $V$ auditory cortical cells and effects of intracellular GABA(A) blockade. J. Neurophysiol. 83, 2626-2638. https://doi.org/10.1152/jn.2000.83.5.2626

Hubel, D.H., Henson, C.O., Rupert, A., Galambos, R., 1959. "Attention" units in the auditory cortex. Science (80-. ). 129, 1279-1280. https://doi.org/10.1126/science.129.3358.1279

Insel, N., Barnes, C.A., 2015. Differential activation of fast-spiking and regularfiring neuron populations during movement and reward in the dorsal medial frontal cortex. Cereb. Cortex 25, 2631-2647. https://doi.org/10.1093/cercor/bhu062

Irvine, D.R.F., 2018a. Plasticity in the auditory system. Hear. Res. https://doi.org/10.1016/j.heares.2017.10.011 Irvine, D.R.F., 2018b. Auditory perceptual learning and changes in the conceptualization of auditory cortex. Hear. Res. https://doi.org/10.1016/j.heares.2018.03.011

Ishishita, Y., Kunii, N., Shimada, S., Ibayashi, K., Tada, M., Kirihara, K., Kawai, K., Uka, T., Kasai, K., Saito, N., 2019. Deviance detection is the dominant component of auditory contextual processing in the lateral superior temporal gyrus: A human ECoG study. Hum. Brain Mapp. 40, 1184-1194. https://doi.org/10.1002/hbm.24438

Jaramillo, S., Zador, A.M., 2011. The auditory cortex mediates the perceptual 
effects of acoustic temporal expectation, in: Nature Neuroscience. Nat Neurosci, pp. 246-253. https://doi.org/10.1038/nn.2688

Jones, C.K., Byun, N., Bubser, M., 2012. Muscarinic and nicotinic acetylcholine receptor agonists and allosteric modulators for the treatment of schizophrenia. Neuropsychopharmacology.

https://doi.org/10.1038/npp.2011.199

Kamke, M.R., Brown, M., Irvine, D.R.F., 2005. Basal forebrain cholinergic input is not essential for lesion-induced plasticity in mature auditory cortex. Neuron 48, 675-686. https://doi.org/10.1016/j.neuron.2005.09.014

Kato, H.K., Gillet, S.N., Isaacson, J.S., 2015. Flexible Sensory Representations in Auditory Cortex Driven by Behavioral Relevance. Neuron 88, 10271039. https://doi.org/10.1016/j.neuron.2015.10.024

Kawaguchi, Y., Kondo, S., 2002. Parvalbumin, somatostatin and cholecystokinin as chemical markers for specific GABAergic interneuron types in the rat frontal cortex. J. Neurocytol. 31, 277-287. https://doi.org/10.1023/A:1024126110356

Keller, G.B., Mrsic-Flogel, T.D., 2018. Predictive Processing: A Canonical Cortical Computation. Neuron. https://doi.org/10.1016/j.neuron.2018.10.003 Kilgard, M.P., Merzenich, M.M., 1998. Cortical map reorganization enabled by nucleus basalis activity. Science (80-. ). 279, 1714-1718. https://doi.org/10.1126/science.279.5357.1714

King, A.J., Dahmen, J.C., Keating, P., Leach, N.D., Nodal, F.R., Bajo, V.M., 2011. Neural circuits underlying adaptation and learning in the perception of auditory space. Neurosci. Biobehav. Rev. https://doi.org/10.1016/j.neubiorev.2011.03.008 
Klinkenberg, I., Blokland, A., Riedel, W.J., Sambeth, A., 2013. Cholinergic modulation of auditory processing, sensory gating and novelty detection in human participants. Psychopharmacology (Berl). https://doi.org/10.1007/s00213-012-2872-0

Kostal, L., Lansky, P., Stiber, M., 2018. Statistics of inverse interspike intervals: The instantaneous firing rate revisited. Chaos $28,1-8$. https://doi.org/10.1063/1.5036831

Kremláček, J., Kreegipuu, K., Tales, A., Astikainen, P., Põldver, N., Näätänen, R., Stefanics, G., 2016. Visual mismatch negativity (vMMN): A review and meta-analysis of studies in psychiatric and neurological disorders. Cortex. https://doi.org/10.1016/j.cortex.2016.03.017

Kuhn, J., Hardenacke, K., Lenartz, D., Gruendler, T., Ullsperger, M., Bartsch, C., Mai, J.K., Zilles, K., Bauer, A., Matusch, A., Schulz, R.J., Noreik, M., Bührle, C.P., Maintz, D., Woopen, C., Häussermann, P., Hellmich, M., Klosterkötter, J., Wiltfang, J., Maarouf, M., Freund, H.J., Sturm, V., 2015. Deep brain stimulation of the nucleus basalis of Meynert in Alzheimer's dementia. Mol. Psychiatry. https://doi.org/10.1038/mp.2014.32

Kuhn, Jens, Hardenacke, K., Shubina, E., Lenartz, D., Visser-Vandewalle, V., Zilles, K., Sturm, V., Freund, H.J., 2015. Deep brain stimulation of the nucleus basalis of meynert in early stage of Alzheimer's dementia. Brain Stimul. https://doi.org/10.1016/j.brs.2015.04.002

Kumbhare, D., Palys, V., Toms, J., Wickramasinghe, C.S., Amarasinghe, K., Manic, M., Hughes, E., Holloway, K.L., 2018. Nucleus basalis of Meynert stimulation for dementia: Theoretical and technical considerations. Front. Neurosci. https://doi.org/10.3389/fnins.2018.00614 
Lee, C.C., Winer, J.A., 2008. Connections of cat auditory cortex: I.

Thalamocortical system. J. Comp. Neurol.

https://doi.org/10.1002/cne.21611

Liljenstrom, H., Hasselmo, M.E., 1995. Cholinergic modulation of cortical oscillatory dynamics. J. Neurophysiol. 74, 288-297. https://doi.org/10.1152/jn.1995.74.1.288

Loewi, O., 1921. Über humorale übertragbarkeit der Herznervenwirkung - I. Mitteilung. Pflugers Arch. Gesamte Physiol. Menschen Tiere 189, 239-242. https://doi.org/10.1007/BF01738910

Lucas-Meunier, E., Fossier, P., Baux, G., Amar, M., 2003. Cholinergic modulation of the cortical neuronal network. Pflugers Arch. Eur. J. Physiol. https://doi.org/10.1007/s00424-002-0999-2

Ma, X., Suga, N., 2005. Long-term cortical plasticity evoked by electric stimulation and acetylcholine applied to the auditory cortex. Proc. Natl. Acad. Sci. U. S. A. 102, 9335-9340. https://doi.org/10.1073/pnas.0503851102

Malmierca, M.S., 2015. Auditory System, in: The Rat Nervous System: Fourth Edition. https://doi.org/10.1016/B978-0-12-374245-2.00029-2

Malmierca, Manuel S., Cristaudo, S., Pérez-González, D., Covey, E., 2009. Stimulus-specific adaptation in the inferior colliculus of the anesthetized rat. J. Neurosci. 29, 5483-5493. https://doi.org/10.1523/JNEUROSCI.415308.2009

Malmierca, Manuel S, Cristaudo, S., Pérez-González, D., Covey, E., 2009. Behavioral/Systems/Cognitive Stimulus-Specific Adaptation in the Inferior Colliculus of the Anesthetized Rat. Soc Neurosci. 
https://doi.org/10.1523/JNEUROSCI.4153-08.2009

Malmierca, M.S., Sanchez-Vives, M. V., Escera, C., Bendixen, A., 2014.

Neuronal adaptation, novelty detection and regularity encoding in audition.

Front. Syst. Neurosci. https://doi.org/10.3389/fnsys.2014.00111

Mansour, S., Magnan, J., Haidar, H., Nicolas, K., Louryan, S., Mansour, S.,

Magnan, J., Haidar, H., Nicolas, K., Louryan, S., 2013. Middle Ear

Contents, in: Comprehensive and Clinical Anatomy of the Middle Ear.

Springer Berlin Heidelberg, pp. 49-81. https://doi.org/10.1007/978-3-642-

36967-4_3

Marshall, L., Mathys, C., Ruge, D., de Berker, A.O., Dayan, P., Stephan, K.E.,

Bestmann, S., 2016. Pharmacological Fingerprints of Contextual

Uncertainty. PLoS Biol. 14. https://doi.org/10.1371/journal.pbio.1002575

Martins, A.R.O., Froemke, R.C., 2015. Coordinated forms of noradrenergic

plasticity in the locus coeruleus and primary auditory cortex. Nat. Neurosci.

18, 1483-1492. https://doi.org/10.1038/nn.4090

Matthews, N., Todd, J., Mannion, D.J., Finnigan, S., Catts, S., Michie, P.T., 2013. Impaired processing of binaural temporal cues to auditory scene analysis in schizophrenia. Schizophr. Res. 146, 344-348.

https://doi.org/10.1016/j.schres.2013.02.013

May, P., Tiitinen, H., Ilmoniemi, R.J., Nyman, G., Taylor, J.G., Näätänen, R., 1999. Frequency change detection in human auditory cortex. J. Comput. Neurosci. https://doi.org/10.1023/A:1008896417606

Mendoza, G., Peyrache, A., Gámez, J., Prado, L., Buzsáki, G., Merchant, H., 2016. Recording extracellular neural activity in the behaving monkey using a semichronic and high-density electrode system. J. Neurophysiol. 116, 
563-574. https://doi.org/10.1152/jn.00116.2016

Mesulam, M.M., 2013. Cholinergic circuitry of the human nucleus basalis and its fate in Alzheimer's disease. J. Comp. Neurol.

https://doi.org/10.1002/cne.23415

Metherate, R., 2011. Functional connectivity and cholinergic modulation in auditory cortex. Neurosci. Biobehav. Rev.

https://doi.org/10.1016/j.neubiorev.2010.11.010

Metherate, R., Cox, C.L., Ashe, J.H., 1992. Cellular bases of neocortical activation: Modulation of neural oscillations by the nucleus basalis and endogenous acetylcholine. J. Neurosci. 12, 4701-4711. https://doi.org/10.1523/jneurosci.12-12-04701.1992

Michalareas, G., Vezoli, J., van Pelt, S., Schoffelen, J.M., Kennedy, H., Fries, P., 2016. Alpha-Beta and Gamma Rhythms Subserve Feedback and Feedforward Influences among Human Visual Cortical Areas. Neuron 89, 384-397. https://doi.org/10.1016/j.neuron.2015.12.018

Moore, A.K., Wehr, M., 2013. Parvalbumin-Expressing Inhibitory Interneurons in Auditory Cortex Are Well-Tuned for Frequency. J. Neurosci. 33, 1371313723. https://doi.org/10.1523/JNEUROSCI.0663-13.2013

Moran, R.J., Campo, P., Symmonds, M., Stephan, K.E., Dolan, R.J., Friston, K.J., 2013. Behavioral/Cognitive Free Energy, Precision and Learning: The Role of Cholinergic Neuromodulation. Soc Neurosci. https://doi.org/10.1523/JNEUROSCI.4255-12.2013

Motts, S.D., Schofield, B.R., 2009. Sources of cholinergic input to the inferior colliculus. Neuroscience 160, 103-114. https://doi.org/10.1016/j.neuroscience.2009.02.036 
Mountcastle, V.B., Talbot, W.H., Sakata, H., Hyvärinen, J., 1969. Cortical neuronal mechanisms in flutter-vibration studied in unanesthetized monkeys. Neuronal periodicity and frequency discrimination. J. Neurophysiol. 32, 452-484. https://doi.org/10.1152/jn.1969.32.3.452 Muñoz, W., Rudy, B., 2014. Spatiotemporal specificity in cholinergic control of neocortical function. Curr. Opin. Neurobiol. https://doi.org/10.1016/j.conb.2014.02.015

Muñoz, W., Tremblay, R., Levenstein, D., Rudy, B., 2017. Layer-specific modulation of neocortical dendritic inhibition during active wakefulness. Science (80-. ). 355, 954-959. https://doi.org/10.1126/science.aag2599

Näätänen, R., 2018. Attention and brain function, Attention and Brain Function. https://doi.org/10.4324/9780429487354

Näätänen, R., 2009. Somatosensory mismatch negativity: A new clinical tool for developmental neurological research? Dev. Med. Child Neurol. https://doi.org/10.1111/j.1469-8749.2009.03386.x

Näätänen, R., Gaillard, A.W.K., Mäntysalo, S., 1978. Early selective-attention effect on evoked potential reinterpreted. Acta Psychol. (Amst). https://doi.org/10.1016/0001-6918(78)90006-9

Näätänen, R., Paavilainen, P., Rinne, T., Alho, K., 2007. The mismatch negativity ( $\mathrm{MMN})$ in basic research of central auditory processing: A review. Clin. Neurophysiol. https://doi.org/10.1016/j.clinph.2007.04.026

Näätänen, R., Tervaniemi, M., Sussman, E., Paavilainen, P., Winkler, I., 2001. "Primitive intelligence" in the auditory cortex. Trends Neurosci. https://doi.org/10.1016/S0166-2236(00)01790-2

Näätänen, R., Winkler, I., 1999. The concept of auditory stimulus representation 
in cognitive neuroscience. Psychol. Bull. https://doi.org/10.1037/00332909.125.6.826

Natan, R.G., Briguglio, J.J., Mwilambwe-Tshilobo, L., Jones, S.I., Aizenberg, M., Goldberg, E.M., Geffen, M.N., 2015. Complementary control of sensory adaptation by two types of cortical interneurons. Elife 4 . https://doi.org/10.7554/eLife.09868

Nelken, I., 2014. Stimulus-specific adaptation and deviance detection in the auditory system: experiments and models. Biol. Cybern. https://doi.org/10.1007/s00422-014-0585-7

Netser, S., Zahar, Y., Gutfreund, Y., 2011. Stimulus-specific adaptation: Can it be a neural correlate of behavioral habituation? J. Neurosci. 31, 1781117820. https://doi.org/10.1523/JNEUROSCI.4790-11.2011

Newman, E.L., Gupta, K., Climer, J.R., Monaghan, C.K., Hasselmo, M.E., 2012. Cholinergic modulation of cognitive processing: Insights drawn from computational models. Front. Behav. Neurosci. https://doi.org/10.3389/fnbeh.2012.00024

Nieto-Diego, J., Malmierca, M.S., 2016. Topographic Distribution of StimulusSpecific Adaptation across Auditory Cortical Fields in the Anesthetized Rat. PLoS Biol. 14. https://doi.org/10.1371/journal.pbio.1002397

Noudoost, B., Moore, T., 2011. The role of neuromodulators in selective attention. Trends Cogn. Sci. https://doi.org/10.1016/j.tics.2011.10.006

Ogawa, H., Oka, K., 2015. Direction-Specific Adaptation in Neuronal and Behavioral Responses of an Insect Mechanosensory System. Soc Neurosci. https://doi.org/10.1523/JNEUROSCI.1378-15.2015

Parr, T., Friston, K.J., 2017. Uncertainty, epistemics and active inference. 
royalsocietypublishing.org 14. https://doi.org/10.1098/rsif.2017.0376

Parras, G.G., Nieto-Diego, J., Carbajal, G. V., Valdés-Baizabal, C., Escera, C., Malmierca, M.S., 2017a. Neurons along the auditory pathway exhibit a hierarchical organization of prediction error. Nat. Commun. 8. https://doi.org/10.1038/s41467-017-02038-6

Parras, G.G., Nieto-Diego, J., Carbajal, G. V., Valdés-Baizabal, C., Escera, C., Malmierca, M.S., 2017b. Neurons along the auditory pathway exhibit a hierarchical organization of prediction error. Nat. Commun. 8. https://doi.org/10.1038/s41467-017-02038-6

Paxinos, G., Watson, C., Diego, S., Boston, L., York, N., 1997. The Rat Brainin Stereotaxic Coordinates Academic Press.

Pérez-González, D., Hernández, O., Covey, E., Malmierca, M.S., 2012. GABA(A)-mediated inhibition modulates stimulus-specific adaptation in the inferior colliculus. PLoS One 7, e34297. https://doi.org/10.1371/journal.pone.0034297

Pérez-González, D., Malmierca, M.S., 2014. Adaptation in the auditory system: An overview. Front. Integr. Neurosci. https://doi.org/10.3389/fnint.2014.00019

Pérez-González, D., Malmierca, M.S., 2012. Variability of the time course of stimulus-specific adaptation in the inferior colliculus. Front. Neural Circuits 6. https://doi.org/10.3389/fncir.2012.00107

Pérez-González, D., Malmierca, M.S., Covey, E., 2005. Novelty detector neurons in the mammalian auditory midbrain. Eur. J. Neurosci. https://doi.org/10.1111/j.1460-9568.2005.04472.x

Peyrache, A., Dehghani, N., Eskandar, E.N., Madsen, J.R., Anderson, W.S., 
Donoghue, J.A., Hochberg, L.R., Halgren, E., Cash, S.S., Destexhe, A., 2012. Spatiotemporal dynamics of neocortical excitation and inhibition during human sleep. Proc. Natl. Acad. Sci. U. S. A. 109, 1731-1736. https://doi.org/10.1073/pnas.1109895109

Picciotto, M.R., Higley, M.J., Mineur, Y.S., 2012. Acetylcholine as a Neuromodulator: Cholinergic Signaling Shapes Nervous System Function and Behavior. Neuron. https://doi.org/10.1016/j.neuron.2012.08.036

Pickles, J.O., 2015. Auditory pathways: Anatomy and physiology, in: Handbook of Clinical Neurology. https://doi.org/10.1016/B978-0-444-62630-1.00001-9

Pinto, L., Goard, M.J., Estandian, D., Xu, M., Kwan, A.C., Lee, S.H., Harrison, T.C., Feng, G., Dan, Y., 2013. Fast modulation of visual perception by basal forebrain cholinergic neurons. Nat. Neurosci. 16, 1857-1863. https://doi.org/10.1038/nn.3552

Polack, P.O., Friedman, J., Golshani, P., 2013. Cellular mechanisms of brain state-dependent gain modulation in visual cortex. Nat. Neurosci. 16, 13311339. https://doi.org/10.1038/nn.3464

Polley, D.B., Read, H.L., Storace, D.A., Merzenich, M.M., 2007. Multiparametric auditory receptive field organization across five cortical fields in the albino rat. J. Neurophysiol. 97, 3621-3638. https://doi.org/10.1152/jn.01298.2006

Popelar, J., Groh, D., Pelánová, J., Canlon, B., Syka, J., 2006. Age-related changes in cochlear and brainstem auditory functions in Fischer 344 rats. Neurobiol. Aging 27, 490-500. https://doi.org/10.1016/j.neurobiolaging.2005.03.001

Profant, O., Burianová, J., Syka, J., 2013. The response properties of neurons in different fields of the auditory cortex in the rat. Hear. Res. 
https://doi.org/10.1016/j.heares.2012.11.021

Puckett, A.C., Pandya, P.K., Moucha, R., Dai, W., Kilgard, M.P., 2007. Plasticity in the Rat Posterior Auditory Field Following Nucleus Basalis Stimulation. J. Neurophysiol. 98, 253-265. https://doi.org/10.1152/jn.01309.2006

Recanzone, G.H., Schreiner, C.E., Merzenich, M.M., 1993. Plasticity in the frequency representation of primary auditory cortex following discrimination training in adult owl monkeys. J. Neurosci. 13, 87-103.

https://doi.org/10.1523/jneurosci.13-01-00087.1993

Recasens, M., Gross, J., Uhlhaas, P.J., 2018. Low-Frequency Oscillatory

Correlates of Auditory Predictive Processing in Cortical-Subcortical

Networks: A MEG-Study. Sci. Rep. 8, 14007.

https://doi.org/10.1038/s41598-018-32385-3

Ruhnau, P., Herrmann, B., Schröger, E., 2012. Finding the right control: The

mismatch negativity under investigation. Clin. Neurophysiol. 123, 507-512. https://doi.org/10.1016/j.clinph.2011.07.035

Ruzzoli, M., Pirulli, C., Mazza, V., Miniussi, C., Brignani, D., 2016. The mismatch negativity as an index of cognitive decline for the early detection of Alzheimer's disease. Sci. Rep. 6, 1-11.

https://doi.org/10.1038/srep33167

Safieddine, S., El-Amraoui, A., Petit, C., 2012. The Auditory Hair Cell Ribbon

Synapse: From Assembly to Function. Annu. Rev. Neurosci.

https://doi.org/10.1146/annurev-neuro-061010-113705

Saldaña, E., 2015. All the Way from the Cortex: a Review of Auditory

Corticosubcollicular Pathways. Cerebellum. https://doi.org/10.1007/s12311015-0694-4 
Sarter, M., Givens, B., Bruno, J.P., 2001. The cognitive neuroscience of sustained attention: Where top-down meets bottom-up. Brain Res. Rev. https://doi.org/10.1016/S0165-0173(01)00044-3

Sceniak, M.P., Maclver, M.B., 2006. Cellular actions of urethane on rat visual cortical neurons in vitro. J. Neurophysiol. 95, 3865-3874. https://doi.org/10.1152/jn.01196.2005

Schofield, B.R., 2011. Central Descending Auditory Pathways. https://doi.org/10.1007/978-1-4419-7070-1_9

Schofield, B.R., Coomes, D.L., 2005. Projections from auditory cortex contact cells in the cochlear nucleus that project to the inferior colliculus. Hear. Res. https://doi.org/10.1016/j.heares.2005.03.005

Schreiner, C.E., Polley, D.B., 2014. Auditory map plasticity: Diversity in causes and consequences. Curr. Opin. Neurobiol. https://doi.org/10.1016/j.conb.2013.11.009

Schröger, E., Wolff, C., 1996. Mismatch response of the human brain to changes in sound location. Neuroreport 7, 3005-3008. https://doi.org/10.1097/00001756-199611250-00041

Sedley, W., Gander, P.E., Kumar, S., Kovach, C.K., Oya, H., Kawasaki, H., Howard, M.A., Griffiths, T.D., 2016. Neural signatures of perceptual inference. Elife 5. https://doi.org/10.7554/eLife.11476

Shen, G., Weiss, S.M., Meltzoff, A.N., Marshall, P.J., 2018. The somatosensory mismatch negativity as a window into body representations in infancy. Int. J. Psychophysiol. 134, 144-150. https://doi.org/10.1016/j.ijpsycho.2018.10.013

Shiramatsu, T.I., Kanzaki, R., Takahashi, H., 2013. Cortical mapping of 
mismatch negativity with deviance detection property in rat. PLoS One. https://doi.org/10.1371/journal.pone.0082663

Sikkens, T., Bosman, C.A., Olcese, U., 2019. The Role of Top-Down Modulation in Shaping Sensory Processing Across Brain States: Implications for Consciousness. Front. Syst. Neurosci. https://doi.org/10.3389/fnsys.2019.00031

Soma, S., Shimegi, S., Suematsu, N., Sato, H., 2013. Cholinergic modulation of response gain in the rat primary visual cortex. Sci. Rep. https://doi.org/10.1038/srep01138

Stefanics, G., Astikainen, P., Czigler, I., 2015. Visual mismatch negativity (vMMN): a prediction error signal in the visual modality. Front. Hum. Neurosci. 8, 1074. https://doi.org/10.3389/fnhum.2014.01074

Stefanics, G., Stefanics, G., Kremláček, J., Czigler, I., 2014. Visual mismatch negativity: A predictive coding view. Front. Hum. Neurosci. https://doi.org/10.3389/fnhum.2014.00666

Summerfield, C., Trittschuh, E.H., Monti, J.M., Mesulam, M.M., Egner, T., 2008. Neural repetition suppression reflects fulfilled perceptual expectations. Nat. Neurosci. 11, 1004-1006. https://doi.org/10.1038/nn.2163

Sussman, E., Winkler, I., 2001. Dynamic sensory updating in the auditory system. Cogn. Brain Res. https://doi.org/10.1016/S0926-6410(01)00067-2

Szabó, B.T., Denham, S.L., Winkler, I., 2016. Computational models of auditory scene analysis: A review. Front. Neurosci. https://doi.org/10.3389/fnins.2016.00524

Thiele, A., Bellgrove, M.A., 2018. Neuromodulation of Attention. Neuron. https://doi.org/10.1016/j.neuron.2018.01.008 
Todorovic, A., van Ede, F., Maris, E., de Lange, F.P., 2011. Prior expectation mediates neural adaptation to repeated sounds in the auditory cortex: An MEG study. J. Neurosci. 31, 9118-9123.

https://doi.org/10.1523/JNEUROSCI.1425-11.2011

Tremblay, R., Lee, S., Rudy, B., 2016. GABAergic Interneurons in the Neocortex: From Cellular Properties to Circuits. Neuron. https://doi.org/10.1016/j.neuron.2016.06.033

Ulanovsky, N., Las, L., Farkas, D., Nelken, I., 2005. “ Multiple time scales of adaptation in auditory cortex neurons": Correction.

Ulanovsky, N., Las, L., Nelken, I., 2003. Processing of low-probability sounds by cortical neurons. Nat. Neurosci. 6, 391-398. https://doi.org/10.1038/nn1032 Valdés-Baizabal, C., Carbajal, G. V., Pérez-González, D., Malmierca, M.S., 2020a. Dopamine gates prediction error forwarding in the cortices of the inferior colliculus. PLoS Biol. https://doi.org/10.1101/824656

Valdés-Baizabal, C., Carbajal, G. V., Pérez-González, D., Malmierca, M.S., 2020b. Dopamine modulates subcortical responses to surprising sounds. PLoS Biol. 18, e3000744. https://doi.org/10.1371/journal.pbio.3000744 Valdés-Baizabal, C., Carbajal, G. V., Pérez-González, D., Malmierca, M.S., 2019. Dopamine gates prediction error forwarding in the cortices of the inferior colliculus. bioRxiv 824656. https://doi.org/10.1101/824656

Veit, J., Hakim, R., Jadi, M.P., Sejnowski, T.J., Adesnik, H., 2017. Cortical gamma band synchronization through somatostatin interneurons. Nat. Neurosci. 20, 951-959. https://doi.org/10.1038/nn.4562

Villano, I., Messina, A., Valenzano, A., Moscatelli, F., Esposito, T., Monda, V., Esposito, M., Precenzano, F., Carotenuto, M., Viggiano, A., Chieffi, S., 
Cibelli, G., Monda, M., Messina, G., 2017. Basal forebrain cholinergic system and orexin neurons: Effects on attention. Front. Behav. Neurosci. https://doi.org/10.3389/fnbeh.2017.00010

Von Der Behrens, W., Bäuerle, P., Kössl, M., Gaese, B.H., 2009.

Behavioral/Systems/Cognitive Correlating Stimulus-Specific Adaptation of Cortical Neurons and Local Field Potentials in the Awake Rat. Soc Neurosci. https://doi.org/10.1523/JNEUROSCI.3475-09.2009

Vossel, S., Weidner, R., Driver, J., Friston, K.J., Fink, G.R., 2012.

Deconstructing the architecture of dorsal and ventral attention systems with dynamic causal modeling. J. Neurosci. 32, 10637-10648. https://doi.org/10.1523/JNEUROSCI.0414-12.2012

Weinberger, N.M., 2007. Auditory associative memory and representational plasticity in the primary auditory cortex. Hear. Res. https://doi.org/10.1016/j.heares.2007.01.004

Weinberger, N.M., 2004. Specific long-term memory traces in primary auditory cortex. Nat. Rev. Neurosci. 5, 279-290. https://doi.org/10.1038/nrn1366

Winer, J.A., Prieto, J.J., 2001. Layer V in cat primary auditory cortex (AI): Cellular architecture and identification of projection neurons. J. Comp. Neurol. https://doi.org/10.1002/cne.1183

Winkler, I., Karmos, G., Näätänen, R., 1996. Adaptive modeling of the unattended acoustic environment reflected in the mismatch negativity event-related potential. Brain Res. https://doi.org/10.1016/S00068993(96)01008-6

Wood, K.C., Blackwell, J.M., Geffen, M.N., 2017. Cortical inhibitory interneurons control sensory processing. Curr. Opin. Neurobiol. 
https://doi.org/10.1016/j.conb.2017.08.018

Xu, H., Jeong, H.Y., Tremblay, R., Rudy, B., 2013. Neocortical SomatostatinExpressing GABAergic Interneurons Disinhibit the Thalamorecipient Layer 4. Neuron 77, 155-167. https://doi.org/10.1016/j.neuron.2012.11.004

Yabe, H., Tervaniemi, M., Reinikainen, K., Näätänen, R., 1997. Temporal window of integration revealed by MMN to sound omission. Neuroreport. https://doi.org/10.1097/00001756-199705260-00035

Yaron, A., Jankowski, M.M., Badrieh, R., Nelken, I., 2020. Stimulus-specific adaptation to behaviorally-relevant sounds in awake rats. PLoS One. https://doi.org/10.1371/journal.pone.0221541

Yu, A.J., Dayan, P., 2005. Uncertainty, neuromodulation, and attention. Neuron 46, 681-692. https://doi.org/10.1016/j.neuron.2005.04.026

Yu, A.J., Dayan, P., 2002. Acetylcholine in cortical inference. Neural Networks 15, 719-730. https://doi.org/10.1016/S0893-6080(02)00058-8

Zaborszky, L., Hoemke, L., Mohlberg, H., Schleicher, A., Amunts, K., Zilles, K., 2008. Stereotaxic probabilistic maps of the magnocellular cell groups in human basal forebrain. Neuroimage 42, 1127-1141. https://doi.org/10.1016/j.neuroimage.2008.05.055

Znamenskiy, P., Zador, A.M., 2013. Corticostriatal neurons in auditory cortex drive decisions during auditory discrimination. Nature 497, 482-485. https://doi.org/10.1038/nature12077 


\section{Publications}


Research Paper

\title{
Deviance detection in physiologically identified cell types in the rat auditory cortex
}

\author{
David Pérez-González a, b, Gloria G. Parras ${ }^{\text {a, b }}{ }^{a}$, Camilo J. Morado-Díaz ${ }^{\text {a, b }}$, \\ Cristian Aedo-Sánchez a, b', Guillermo V. Carbajal a, b, Manuel S. Malmierca a, b,c, * \\ Cognitive and Auditory Neuroscience Laboratory (Lab 1), institute of Neuroscience of Castilla y León (INCYL), University of Salamanca, Salamanca, Spain \\ instiute for Biomedical Research of Salamanca (IBSAL). Spain \\ Department of Cell Biology and Pathology, Faculty of Mediane, University of Salamanca, Spain
}

A R T I C L E I N F O

Artide history:

Received 9 January 2020

Received in revised form

7 May 2020

Acrepted 13 May 2020

Available online xxo

Keywords

Predictive coding

Stimulus-specific adaptation

Spike width analysis

Inhibitory

Fast spiking neuron

Regular spinin
A B S T R A C T

Auditory deviance detection is a function of the auditory system that allows reduction of the processing demand for repetitive stimuli while stressing unpredictable ones, which are potentially more informative. Deviance detection has been extensively studied in humans using the oddball paradigm, which evokes an event-related potential known as mismatch negativity (MMN). The same stimulation paradigms are used in animal studies that aim to elucidate the neuronal mechanisms underlying deviance detection. In order to understand the circuitry responsible for deviance detection in the auditory cortex (AC), it is necessary to determine the properties of excitatory and inhibitory neurons separately. Measuring the spike widths of neurons recorded extracellularly from the anaesthetized rat AC, we
classified them as fast spiking or regular spiking units. These two neuron types are generally considered as putative inhibitory or excitatory, respectively. In response to an oddball paradigm, we found that both types of units showed similar amounts of deviance detection overall. When considering each AC field separately, we found that only in A1 fast spiking neurons showed higher deviance detection levels than regular spiking neurons, while in the rest of the fields there was no such distinction. Interpreting these responses in the context of the predictive coding framework, we found that the responses of both types of units reflect mainly prediction error signaling (i.e., genuine deviance detection) rather than repetition suppression.

2020 Elsevier B.V. All rights reserved.

\section{Introduction}

Deviance detection is the capacity of the nervous system to filter out repetitive, non-informative stimuli while stressing unpredictable ones, which may be highly relevant for the individual. In the auditory domain, deviance detection is reflected in the form of an event-related potential known as mismatch negativity (MMN), which has been mostly studied in humans. MMN is evoked using an oddball paradigm, where a sequence of repetitive sounds (standard) is randomly broken by the presentation of a different, rare sound (deviant) (Näätänen et al., 1978). Thus, MMN is the differential response to a given sound when it is presented as a deviant,

\footnotetext{
- Corresponding author. Cognitive and Auditory Neuroscience Laboratory (Lab 1), Institute of Neurosdience of Castilla y León (INCYL), University of Salamanca, Salamanca, Spain

E-mail address: msmeusal.es (M.S. Malmierca).

https://doi.org/10.1016/j.heares.2020.107997

0378-5955/0 2020 Elsevier B. . All rights reserved.
}

compared to when it is presented as a standard. MMN is preattentional (it can be recorded during sleep or even anaesthesia) (Garrido et al., 2009) and has been found altered in some pathologies such as schizophrenia (Michie et al., 2016a, 2016b), Alzheimer's disease (Tsolaki et al., 2017), or autism spectrum disorders (Vlaskamp et al., 2017), so it has potential applications as a diagnostic tool (Näätänen et aL., 2012), and can be used in noncooperative patients such as those in coma.

Several animal studies have recorded the responses of auditory neurons using the same oddball paradigms as in MMN human studies, in order to understand the neuronal basis of MMN. The general agreement in those studies is that some auditory neurons may reduce their response when a sound is presented as standard, while they have a comparatively stronger response to the same sound when it is presented as deviant. This particular type of adaptation is referred to as stimulus-specific adaptation (SSA), and could be interpreted as a neuronal mechanism involved in deviance

Please cite this article as: Pérez-González, D et al., Deviance detection in physiologically identified cell types in the rat auditory cortex, Hearing Research, https://doi.org/10.1016/j.heares.2020.107997 
detection (for a review, see Pérez-González and Malmierca, 2014) Neurons exhibiting high levels of SSA have been found both in the auditory cortex (Nieto-Diego and Malmierca, 2016; Parras et al., 2017; Ulanovsky et al., 2003) and in subcortical nuclei (auditory midbrain: Duque et al., 2012; Malmierca et al., 2009; PérezGonzález et al., 2005; and thalamus: Antunes et al., 2010; Antunes and Malmierca, 2014), being more prominent in nonlemniscal areas (Ayala et al., 2016, 2013; Ayala and Malmierca, 2013; Duque et al., 2012). The role of corticofugal projections is still under study; current evidence suggests that subcortical SSA is not inherited from the cortex, but can be modulated by corticofugal projections (Anderson and Malmierca, 2013; Ayala et al., 2015; Malmierca et al.. 2015). The differential responses to deviant and standard stimuli can be caused simply by neuronal adaptation, reducing the response to the repetitive sound, or by genuine deviance detection, where the appearance of an unexpected sound would result in an enhanced response to the deviant. In order to separate the contributions of each of these mechanisms, responses to deviant and standard stimuli can be compared to the responses to a control sequence accounting for the repetitiveness as well as the expectancy of the stimuli (Carbajal and Malmierca, 2018).

MMN and SSA responses can be best interpreted now under the perspective of the predictive coding framework, which can explain some aspects of these neural responses (Carbajal and Malmierca, 2018; Friston, 2005). According to this theory, past events are used to generate an internal model that is used to predict new events. Those events that do not match the model would generate prediction error (analogous to the previously mentioned genuine deviance detection), which would favor the processing of the nove event and an update of the model. On the other hand, events that match the expectations of the model, such as sequences of repetitive sounds, are already represented in the model and do not require further processing effort, leading to repetition suppression (analogous to the adaptation to repetitive sounds). The predictive coding theory assumes a hierarchy of sensory processing levels, where the outputs of one level would inform the other levels in order to update the internal model.

In order to match the abstract concepts of the deviance detection framework with actual neural elements, and possibly establish a potential neural circuit for deviance detection, it would be helpfu to identify which neurons contribute to each component of deviance detection, such as repetition suppression and prediction error The strong and distinct MMN and SSA responses, as well as the connectivity, microcircuitry and neuronal organization of the auditory cortex $(\mathrm{AC})$ make this region an ideal place to identify such components.

Although there is considerable variation in the organization of the $A C$ across mammals, it is generally accepted that the $A C$ is made of primary fields with clear tonotopic representation of the audible frequencies, surrounded by belt or secondary, high order fields (Malmierca, 2015; Winer et al., 1999). In the rat AC, the primary fields include A1, the anterior (AAF) and the ventral (VAF) fields while the secondary fields include the posterior (PAF) and suprarhinal (SRAF) fields (Pandya et al., 2008; Polley et al., 2007; Profan et al., 2013). A1, AAF and VAF receive inputs predominantly from lemniscal subcortical areas, while SRAF and PAF receive inputs from non-lemniscal areas (Smith et al., 2012). As in other sensory cortices, the rat AC is made of 6 layers, each with a particular set of cell types. Excitatory pyramidal neurons are mainly located in layers III and V, although some 'spiny' stellate excitatory neurons also occur in layer IV, while different types of inhibitory in terneurons are found in layers I-VI (Budinger and Kanold, 2018) While these two groups of neurons are readily differentiable using histological techniques, their identification using extracellula electrophysiological recordings is not a trivial issue. However, recording from the somatosensory cortex of unanesthetized macaques, Mountcastle et al. (1969) proposed that pyramidal neuron have wide spikes, while other non-pyramidal cortical neurons (which they refer to as potential Golgi type II neurons; likely these neurons correspond to the basket neumns that are known to be inhibitory and a possesses a stellate like morphology but lack spines) have 'thin spikes'. Since then, multiple studies in differen cortical areas have used similar classifications (rat: Hefti and Smith, 2000; cat AC: Atencio and Schreiner, 2008; mouse AC: Chen et al., 2015; Natan et al., 2015; rat medial prefrontal cortex: Baeg et ab, 2001; rat somatosensory cortex: Andermann et al., 2004; Bruno and Simons, 2002; macaque motor cortex: Mendoza et al., 2016; human middle temporal gyrus: Peyrache et al., 2012) where regular spiking (RS) units are considered as putative excitatory neurons while fast spiking (FS) units are considered inhibitory neurons. Hence, cortical neurons can be categorized as putative excitatory and inhibitory based on the shape of their spike waveforms and their firing rates.

In the present study we recorded neurons from the $\mathrm{AC}$ of the anaesthetized rat and classified them as putative excitatory or inhibitory units. We aimed to test whether they have different deviance detection properties. Results show that overall, deviance detection occurs in both excitatory and inhibitory neurons.

\section{Material and methods}

\subsection{Data collection}

This study analyses the spike waveforms from a data set of 282 neuronal recordings, originally collected for other studies regarding neuronal adaptation and predictive coding in the AC. Some of these original studies are already published (Parras et al., 2019, 2017). Al methodological procedures were approved by the Bioethics Committee for Animal Care of the University of Salamanca (USAL-ID195) and performed in compliance with the standards of the European Convention ETS 123, the European Union Directive 2010/63 EU and the Spanish Royal Decree 53/2013 for the use of animals in scientific research.

\section{Surgical procedures}

The recordings were performed in 86 urethane-anesthetized female Long Evans adult rats (body weight $200-250 \mathrm{~g}$ ). For a ful description of the surgical procedures, see (Parras et al., 2017). In summary, animals were anesthetized (urethane $1.5 \mathrm{~g} / \mathrm{kg}$ i.p. and $0.5 \mathrm{~g} / \mathrm{kg}$ for supplementary doses when needed). Depth of anaesthesia was assessed by pedal reflex. Body temperature was monitored by a probe and maintained by a thermal blanket. Normal hearing was assessed by recording the auditory brainstem response (ABR; 0.1 ms clicks, 10-90 dB). The trachea was cannulated to allow artificial ventilation, while expired $\left[\mathrm{CO}_{2}\right]$ was monitored using a capnograph. Then, the animals were placed in a stereotaxic frame, equipped with modified ear bars that allowed the placement of speakers for auditory stimulation. A craniotomy was performed to expose the left $\mathrm{AC}$, and the underlying dura was removed. The exposed cortex was then covered with a layer of agar to prevent desiccation. A tungsten microelectrode was placed orthogonal to the brain surface, and advanced by means of a piezoelectric micromanipulator (Sensapex), in order to record the extracellular activity of AC single neurons. The displacement of the micromanipulator was used to measure the depth of the electrode tip relative to the brain surface. 


\subsection{Acoustic stimulation}

Both acoustic stimuli generation and neuronal potential recordings were performed using a RZ6 Multi I/O Processor (TuckerDavis Technologies), controlled by custom software created with MATLAB (MathWorks) and OpenEx Suite (Tucker-Davis Technologies, TDT). Acoustic stimuli were delivered monaurally to the ear contralateral to the recording side through calibrated speakers in a close-field configuration. The output of the system had a flat spectrum at $76 \mathrm{~dB} \mathrm{SPL}( \pm 3 \mathrm{~dB})$ between $500 \mathrm{~Hz}$ and $45 \mathrm{kHz}$, and the second and third harmonic components in the signal were $\geq 40 \mathrm{~dB}$ below the level of the fundamental at the highest output level. Stimuli consisted of $75 \mathrm{~ms}$ pure tones $(0.5-45 \mathrm{kHz})$, including $5 \mathrm{~ms}$ rise/fall ramps. The presentation rate for the majority of units was 4 stimuli/s, however for some recordings it was $3 / \mathrm{s}(27 \%)$ or $2 / \mathrm{s}$ (13\%), due to the characteristics of the original experiments. The frequency response area was measured for each neuron, presenting tones of different frequencies $(0.5-45 \mathrm{kHz})$ and intensities in random order (3-5 repetitions of each frequency-intensity combination), and was used to estimate the minimum threshold (i.e. the lowest SPL able to evoke a response) and the characteristic frequency (i.e., the tone frequency able to evoke a response the minimum threshold).

After that, pure tones were presented in oddball sequences to test for the adaptation and deviance detection properties of the neuron. For that, a pair of pure tones $\left(f_{1}, f_{2}\right)$ within the frequency response area of the neuron were chosen, $\sim 0.5$ octaves apart, both at the same sound level (10-30 dB above minimum threshold). Each oddball sequence, which included 400 stimuli, consisted of a repetitive tone (standard, $90 \%$ probability), occasionally replaced by a different tone (deviant, $10 \%$ probability), in a pseudorandom manner. The sequence always started with at least 10 standard tones, and each deviant tone was preceded by at least 3 standard tones. On a subsequent oddball sequence, the relative probabilities of $f_{1}$ and $f_{2}$ were exchanged, in order to compensate for the neuron responding differently to each sound frequency, so we could compare the responses to each frequency when it was presented as a standard with the responses to the same frequency when it was presented as a deviant. The differential response to standard and deviant tones is known as stimulus-specific adaptation (SSA), which is considered to reflect two different components: repetition suppression and deviance detection (Carbajal and Malmierca, 2018).

In order to break down both components of SSA, we used the many-standards and the cascade control sequences, which lack the stimulus repetition of the oddball sequence. Both sequences were also 400 stimuli long, and featured pure tones at the same presentation rate, duration and sound level as in the oddball sequence. In both cases 10 frequencies were used (the same in both control sequences), including those in the oddball sequence, and the separation between two consecutive frequencies was also 0.5 octaves. In the many-standards sequence all 10 frequencies were presented randomly, with the same probability of occurrence ( $10 \%$ each tone), with the restriction that all tones must be presented before the next repetition. On the other hand, in the cascade sequences the tones were sorted by increasing or decreasing frequency, and the tone preceding the probe tone was always the same as in the corresponding oddball sequence.

\subsection{Electrophysiological recording}

Extracellular neuronal potentials were recorded using custommade glass-coated tungsten microelectrodes (1-4 M 2 impedance at $1 \mathrm{kHz}$ ). The signal was digitized at $25 \mathrm{kHz}$ by a RA16PA Medusa preamplifier (TDT) and sent to the RZ6 for further processing and visualization. Potentials were band-pass filtered between 0.5 and $3 \mathrm{kHz}$. Initial spike discrimination was done manually setting a voltage threshold at a level that included action potential events but excluded the baseline noise, by a trained experimenter. These events were stored for further offline processing.

\subsection{Analysis of spike waveforms}

The first step of offline processing was spike sorting, which allowed removal of recorded artifacts and noise, and occasionally separate single units. Spike sorting was performed on OpenSorter (TDT), using a supervised Bayesian procedure. Spike waveforms were aligned on the trough. The mean waveform of the spikes of each unit was calculated, and the following measurements were made on the average waveform: time and voltage of the peak and trough, spike amplitude (peak-to-trough) and spike half-width (spike width at $50 \%$ of the peak-to-trough amplitude; hereinafter "spike width") (Fig. 1A). We used the peak-to-trough signal to noise ratio (SNR) as an indicator of the quality of the spikes. The SNR was calculated as the difference between the maximum and minimum voltages, divided by the standard deviation of the background noise (when there were no spikes). Only units with an SNR $>5$ were included in the study (Fig. 1B). The average number of spikes per sorted unit was $3207 \pm 4765$ spikes (range: $21-35610$ spikes) (Fig. 1C); about $95 \%$ of the units included more than 200 spikes.

Interspike intervals were measured over the oddball sequences for each unit, and were expressed as its reciprocal, the instantaneous firing rate (Kostal et al., 2018). We then calculated the distribution of instantaneous firing rates for each unit, and took as reference for the neuronal discharge the values of the 50th, 25th, 10 th, 5 th and 1 st percentiles, in order to account for the average as well as for the fastest firing rates that each neuron could achieve, respectively.

\subsection{Statistical analysis}

All statistical analyses were performed in MATLAB. Since most value distributions did not hold the normality assumption (both by visual inspection and by the results of the Lilliefors normality test), non-parametric tests were employed in this study. We used the Wilcoxon rank-sum test to check whether two independent samples came from distributions with equal medians. The sign test was used to determine whether the median of a group was different from zero. When performing comparisons between two groups (or comparing one group to zero) multiple times (e.g., Fig. 4F, Fig. 5), the resulting p-values were corrected using the Holm-Bonferroni method. For comparisons of distributions between more than two groups (e.g., all the comparisons between cortical fields), the Kruskal-Wallis test was used; post hoc comparisons to find differences between groups were made using the Bonferroni method. The average values of the distributions are expressed as mean $\pm S D$ unless indicated otherwise. Null hypotheses were rejected at a significance level of 0.05 .

We compared the response to each test stimulus when it adopted the role of a standard (STD) or deviant (DEV) tone in an oddball sequence, and also when it was part of a control sequence (CTR). For the subsequent analyses we used the cascade sequence as control, since the responses to the many-standards sequence were very similar (Parras et al., 2017). Baseline-corrected spike count responses of a neuron to the same tone in the three conditions (DEV, STD, CTR) were normalized using the formulas:

$D E V_{\text {Normalized }}=D E V / N$ 

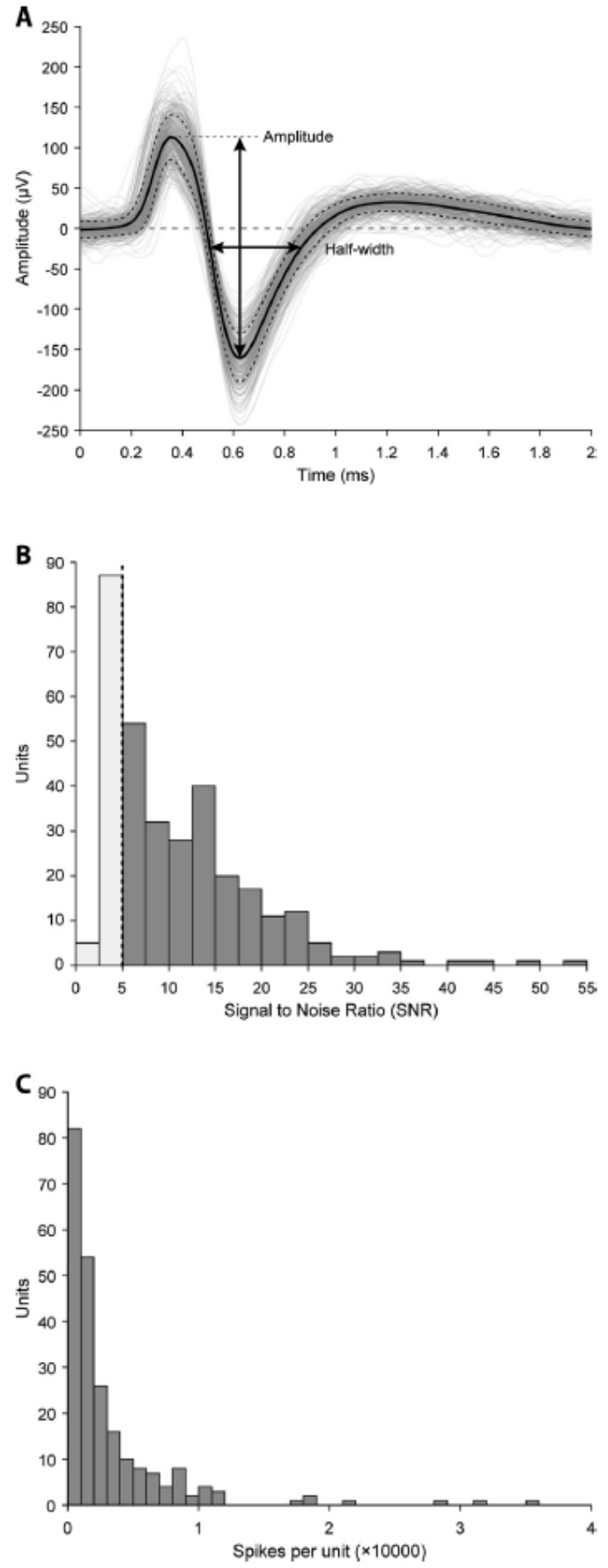

Fig. 1 Spike width measurement A) The spikes for each isolated unit were aligned on the trough. The mean (black solid line) and standard deviation (dotted black lines) of the spikes of each unit was calculated. Based on the mean waveform, we measured the peak-to-trough amplitude (vertical line) and spike half-width (spike width at $50 \%$ of
$S T D_{\text {Normalized }}=S T D / N$

$C T R_{\text {Normaitzed }}=C T R / N$

where

$N=\sqrt{D E V^{2}+S T D^{2}+C T R^{2}}$

is the Euclidean norm of the vector (DEV, STD, CTR) defined by the three responses, which can take values in the range $0-1$. From these normalized responses, the indexes of Neuronal Mismatch, Repetition Suppression, and Prediction Error were calculated in the following way:

Index of Neuronal Mismatch $=D E V_{\text {Normatized }}-S T D_{\text {Normalized }}$

Index of Repetition Suppression $=C T R_{\text {Normalized }}-S T D_{\text {Normalized }}$

Index of Prediction Error $=D E V_{\text {Nomalized }}-C T R_{\text {Normalized }}$

These indexes always range between -1 and 1 , and allow the separation of neuronal mismatch into repetition suppression and prediction error:

Index of Neuronal Mismatch = Index of Repetition Suppression + Index of Prediction Error

The index of neuronal mismatch is largely equivalent to the typical "SSA index", commonly used in previous studies of SSA in single units (Casado-Román et al., 2019; Parras et al., 2019, 2017).

The spontaneous firing rates were measured in the periods between stimuli (during the period of $50 \mathrm{~ms}$ before each stimulus). which were pooled for each sequence. In contrast to measuring the spontaneous activity before each sequence, this method captures better the potential activity drifts that may occur during the sequences. On the other hand, in some cases we may be overestimating the spontaneous activity, since long latency or sustained responses evoked by the previous stimulus may occasionally fall with in that period, but in general this was not the case. Some examples of PSTHs of recordings such as those included in this study are shown in Parras et al. (2019, 2017).

\section{Results}

\subsection{Fast spiking and regular spiking neurons}

We performed 282 recordings from the AC of 86 urethaneanaesthetized rats (median: 2 sites per animal, range: $1-18$ ). Using spike sorting, we were able to collect 323 well isolated single units (241, 37 and 4 sites yielded 1, 2 and 3 units per site, respectively) and measure their spike waveforms. Only units with an SNR $>5$ were included in the subsequent analyses $(n=231)$. Fig. $2 A$ shows the distribution of spike widths in our sample. The average spike width was $0.385 \pm 0.263 \mathrm{~ms}$ (range: $0.134-1.353$ ). These units were classified as regular spiking (RS) or fast spiking (FS) depending on the shape of the spike width distribution, which has a bimodal appearance with a prominent peak towards narrow spike widths and a spread plateau towards wider spike widths. According to this distribution, we set a cut-off at $0.35 \mathrm{~ms}$; units with spike

the peak-to-trough amplitude). B) Distribution of the peak-to-trough signal to noise ratio (SNR). Only units with an SNR $>5$ were included in the study (dotted line). C) Distribution of the number of spikes per unit. About $95 \%$ of the units included more than 200 spikes. 
widths larger than $0.35 \mathrm{~ms}$ were classified as RS (Fig. 2A, orange), and the rest, i.e, units with spike widths shorter than $0.35 \mathrm{~ms}$, as FS (Fig. 2A, blue). In our sample, 60 (26\%) units were classified as RS, and the rest as FS $(\mathrm{n}=171,74 \%)$. The average spike width of RS units was $0.779 \pm 0.230 \mathrm{~ms}$, while that of FS units was $0.247 \pm 0.039 \mathrm{~ms}$ (Fig. 2B). In agreement with previous studies, as we describe next, units classified using this criterion also showed different firing rates.

The neuronal responses were recorded during auditory stimulation using an oddball paradigm, which consists of a sequence of repeated tones (standards), where $10 \%$ of the occurrences are replaced by a deviant tone. This type of sequence tends to produce adaptation of the firing rate during the repetitions of the standard tones and an increased firing rate whenever a deviant tone occurs. We measured the firing rates in the periods between stimul (during the period of $50 \mathrm{~ms}$ before each stimulus), in order to estimate the spontaneous firing rates, but there were no differences between RS and FS units ( $p=0.129$, Wilcoxon rank-sum test).

To better analyse the varying firing rates during the oddbal sequences, we calculated the distribution of interspike intervals for each unit, and from there we derived the distribution of instantaneous firing rates. FS units showed on average larger firing rates than RS units for the 25th, 10th $(p<0.01)$, 5th and 1st percentiles $(p<0.001$ ), but not for the 50th percentile (Wilcoxon rank-sum test, Holm-Bonferroni corrected) (Fig. 2C). This result indicates that FS units are able to achieve higher firing rates than RS units given the proper conditions. The 1st and 5th percentiles of the distribution of firing rates are better indicators of the faster firing rates that a neuron can achieve in near-optimal conditions, since other measurements such as the median (50th percentile) would include a larger proportion of spikes that occur during lessfavourable conditions, e.g. when there is no stimulus present or when the neuron is under the effect of adaptation (see Supplementary Fig. 1).

\subsection{Location in auditory cortex fields}

Based on stereotaxic coordinates and response characteristics, we assigned 205 units to any of the five main fields of the rat $A C$ (Fig. 3A). Thus, 111 units were located in lemniscal fields (A1, $\mathrm{n}=66 ; \mathrm{VAF}, \mathrm{n}=33 ; \mathrm{AAF}, \mathrm{n}=12$ ) and 94 in non-lemniscal fields (SRAF, $\mathrm{n}=47$; PAF, $\mathrm{n}=47$ ). Lemniscal fields contained a larger proportion of FS units ( $81 \%$ across all lemniscal fields; $A 1,85 \%$; VAF, $70 \%$, AAF, 92\%) than non-lemniscal fields (65\% across all nonlemniscal fields; SRAF, $64 \%$; PAF, $66 \%$ ). When comparing the spike widths of both types of units across AC fields, we found no differences for RS units (Kruskal-Wallis, $\chi^{2}(4, N=54)=4.04$ $p=0.401$ ) nor for FS units (Kruskal-Wallis, $\chi^{2}(4, N=151)=8.54$ $p=0.074)$ (Fig. 3B)

\subsection{Spike amplitude}

To look for differences between RS and FS units, we measured the peak-to-trough amplitude (see Fig. 1A) of the average spike waveforms. We found that RS units had larger amplitudes than FS spikes. The peak-to-trough amplitude of RS units $(571.76 \pm 510.59 \mu \mathrm{V})$ was much larger than the amplitude of FS units $(147.61 \pm 93.55 \mu \mathrm{V} ; p<0.001$, Wilcoxon rank-sum test (Fig. 3C). The spike amplitudes were similar among AC fields for RS units (Kruskal-Wallis, $\chi^{2}(4, N=54)=3.27, p=0.514$ ) and FS units (Kruskal-Wallis, $\chi^{2}(4, N=151)=1.68, p=0.514$ ) (Fig. 3D).

Similarly, the signal-to-noise ratio(SNR) of RS units (20.2 \pm 10.3$)$ was significantly larger than the SNR of FS units (11.6 \pm 5.4 $p<0.001$, Wilcoxon rank-sum test) (Supplementary Fig. 2A). As happened in the case of the spike amplitude, there were no differences in the SNR between AC fields for RS units (KruskalWallis, $\left.\chi^{2}(4, N=54)=4.05, p=0.400\right)$ nor for FS units (KruskalWallis, $\chi^{2}(4, N=151)=0.31, p=0.989$ ) (Supplementary Fig. 2B).

\subsection{Cortical layer location}

In order to check whether RS and FS units had any preferential location across the cortical layers, we recorded the depth of the electrode tip from the brain surface for 202 units. The most superficially located unit was recorded at $115 \mu \mathrm{m}$, while the deepest unit was found at $1170 \mu \mathrm{m}$ (mean: $584 \pm 247 \mu \mathrm{m}$ ). RS and FS units were found at similar depths $(596 \pm 238$ vs. $579 \pm 251 \mu \mathrm{m}$ respectively; $p=0.800$, Wilcoxon rank-sum test) (Fig. 4A).

In order to estimate which layers were more likely to contain RS or FS units, we calculated the density of units in each layer, in terms of units per $100 \mu \mathrm{m}$ (based on our sample of 202 located neurons) (Fig. 4B, left). The borders between the cortical layers were based on the depths previously reported in rat by Games and Wine (1988). We found a higher density of RS units within the approximate limits of layers III-V $(5.8-12$ units per $100 \mu \mathrm{m})$, while FS units spread from layer II to layer V (14-18.9 units per $100 \mu \mathrm{m})$. The estimated density of FS units was larger than RS units in all layers.

We also calculated the unit density in supragranular (layers I-III) and infragranular layers (layers V-VI) (Fig. 4B, right) separately. The unit density on both sides of the granular layer was very similar for both RS units ( 4.2 vs 3.3 units per $100 \mu \mathrm{m}$, respectively) and FS units (12.4 vs 11.9 units per $100 \mu \mathrm{m}$, respectively).

There were no significant differences between $\mathrm{AC}$ fields in the depth at which RS units (Kruskal-Wallis, $\chi^{2}(4, N=54)=9.30$ $p=0.054)$ nor FS units (Kruskal-Wallis, $\chi^{2}(4, N=148)=5.20$, $p=0.267$ ) were recorded (Fig. 4C).

\subsection{Adapting properties of fast spiking and regular spiking neurons}

From the original sample, we recorded the responses of 211 neurons to an oddball paradigm and the corresponding cascade and many-standards control sequences. Previous works have established that both control sequences produce similar results (Parras et al., 2017; Wiens et al., 2019); hence, in the present study we only used the cascade control, since it has been argued to be more adequate because it not only controls for the presentation rate, as the many-standards condition does, but also for the refractoriness of the response and repetition suppression effects due to the predictability of cascade tones (Ruhnau et al., 2012).

The index of neuronal mismatch indicates how much a neuron responds to deviant stimuli rather than to standard stimuli (see Material and methods); the average for all units was $0.565 \pm 0.314$ The medians for both RS and FS units were larger than zero (both $p<0.001$, sign test), and there were no statistical differences in the index of neuronal mismatch between both groups $(0.512 \pm 0.386 \mathrm{vs}$ $0.584 \pm 0.283 ; p=0.414$, Wilcoxon rank-sum test) (Fig. 5A). However, when we analysed each individual AC field, we found that in A1 the index of neuronal mismatch of FS units was larger than that of RS units $(0.574 \pm 0.268$ vs $0.230 \pm 0.270, p=0.013$. Wilcoxon rank-sum test). The median index of neuronal mismatch was larger than zero in all AC fields for FS units (A1, $p<0.001$; VAF, $p<0.001$; AAF, $p=0.005$; SRAF, $p<0.001$; PAF, $p<0.001$ ), but only in SRAF and PAF for RS units (A1, $p=0.688$; VAF, $p=0.065$; AAF, $p=1.000$ SRAF, $p<0.001$; PAF, $p=0.017$ ). However, there were significant differences in the index of neuronal mismatch between the AC fields for RS (Kruskal-Wallis, $\chi^{2}(4, N=54)=10.91, p=0.028$ ), but not for FS units (Kruskal-Wallis, $\chi^{2}(4, N=151)=6.93, p=0.140$ ). Post hoc comparisons indicated that the average in dex of neuronal mismatch was significantly lower for RS units in A1 $(0.230 \pm 0.270)$ than in PAF or SRAF $(0.526 \pm 0.534, p=0.029 ;$ and $0.626 \pm 0.255, p=0.021$ 

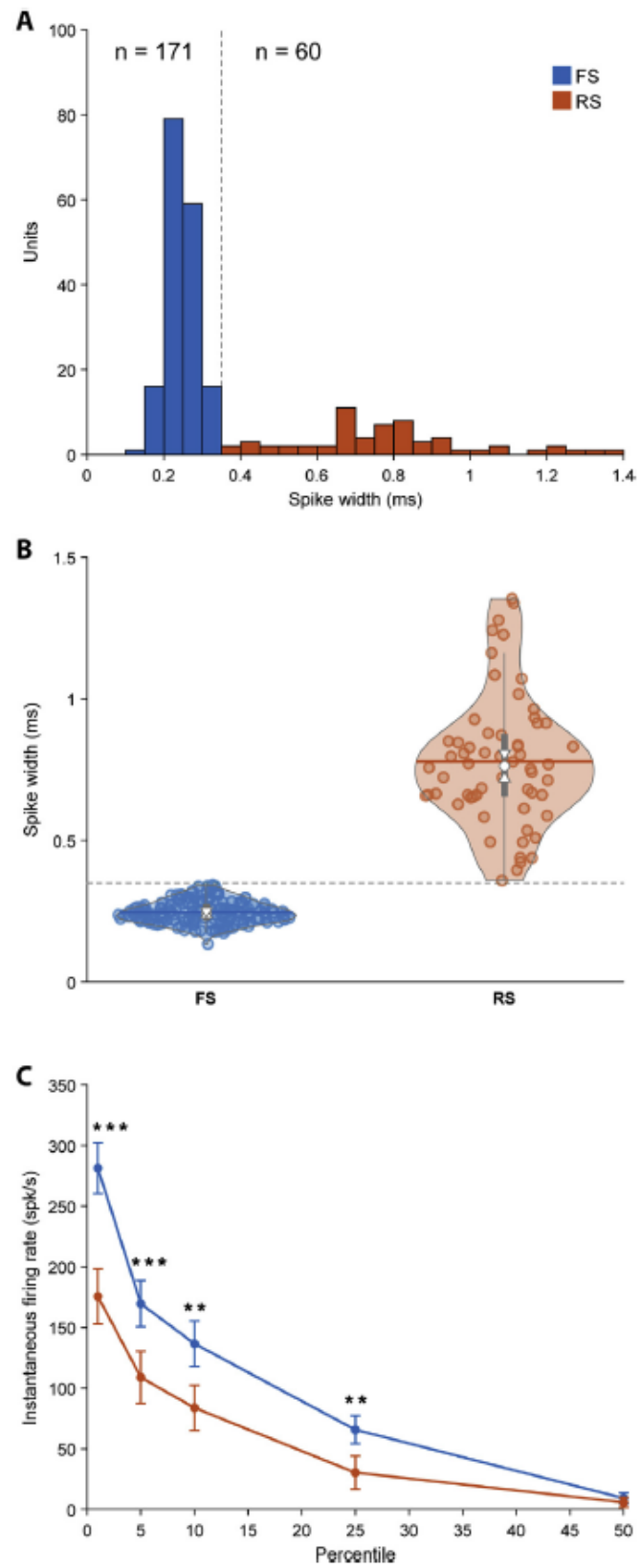

Fig. 2. Classification of units based on spike width and firing rate. A) Distribution of spike widths measured in our sample. Units with a spike width larger than $0.35 \mathrm{~ms}$ (dashed grey line) were classified as regular spiking (RS, orange), while the rest were dassified as fast spiking ( $\mathrm{F}$, blue). B) Violin plot showing the distribution of spike widths in RS and FS units. In this and similar violin plots, the horizontal solid line indicates the mean of the distribution, while the white circle indicates the median. The thick grey bar expands from the first (Q1) to the third quartile (Q3). and the whiskers show the range of lower and higher adjacent values (i.e. values within $1.5 \mathrm{IQR}$ below Q1 or above Q3, respectively. The triang les indicate a $95 \%$ confidence interval for the respectively) (Fig. 5B).

The index of repetition suppression is a measurement of neurona adaptation to standard stimuli compared to a cascade contro sequence (see Material and methods); the average for all units was $0.162 \pm 0.220$. The medians for both RS and FS units were larger than zero (both $p<0.001$, sign test), and there were no statistical differences in the index of repetition suppression between both groups $(0.178 \pm 0.300$ vs $0.157 \pm 0.183 ; p=0.632$ Wilcoxon ranksum test) (Fig. 5C). Similarly, there were no differences between RS and FS units when looking at each individual AC field. The median index of repetition suppression was larger than zero in all $A C$ fields except AAF for FS units (A1, $p<0.001$; VAF, $p<0.001$; AAF, $p=0.262$; SRAF, $p<0.001$; PAF, $p<0.020$ ), but only in SRAF for RS units (A1, $p=1.000$; VAF, $p=0.328$; AAF, $p=1.000$; SRAF, $p=0.016$; PAF, $p=0.106)$. There were differences in the index of repetition suppression among AC fields for RS units (Kruskal-Wallis, $\chi^{2}$ (4. $N=54$ ) $=9.53, p=0.049$ ) but not for FS units (Kruskal-Wallis, $\chi^{2}$ (4 $N=151$ ) $=7.46, p=0.114$ ) (Fig. 5D). However, post hoc comparisons did not find any differences between groups for RS units, although the comparison between A1 and SRAF was close to the level of significance $(p=0.057)$.

The index of prediction error indicates how much does a neuron respond to deviant stimuli as compared to a cascade control sequence (see Material and methods); the average for all units was $0.403 \pm 0.357$. The medians for both RS and FS units were larger than zero (both $p<0.001$, sign test), and there were no statistical differences between both groups $(0.335 \pm 0.431$ vs $0.427 \pm 0.324$; $p=0.310$, Wilcoxon rank-sum test) (Fig. 5E). Similarly, there were no differences between RS and FS units when looking at each individual AC field. The median index of prediction error was larger than zero in all AC fields except VAF for FS units ( $A 1, p<0.001$; VAF, $p=0.173 ; \mathrm{AAF}, p=0.007$; SRAF, $p<0.001$; PAF, $p=0.002$ ), and in no fields for RS units (A1, $p=0.328$; VAF, $p=0.219$; AAF, $p=1.000$; SRAF, $p=0.196$; PAF, $p=0.128$ ). The index of prediction error was similar in all the AC fields, for both RS units (Kruskal-Wallis, $\chi^{2}(4$, $N=54)=5.73, p=0.220$ ) and FS units (Kruskal-Wallis, $\chi^{2}(4$ $N=151)=1.89, p=0.755$ ) (Fig. 5F).

Altogether, while all the indexes tended to be larger than zero in the individual AC fields for FS units but not for RS units (with the few exceptions indicated before), we only found significant differences between these two types of units in A1. A1 was the only field where the index of neuronal mismatch was significantly larger for FS units than for RS units, probably due to a larger index of prediction error, which was close to reach statistical significance $(0.453 \pm 0.307$ vs $0.156 \pm 0.370, p=0.051$, Wilcoxon rank-sum test $)$

Given the non-significant differences in the indexes of repetition suppression and prediction error for RS units between A1 and SRAF/ PAF (Fig. 5D,F), it cannot be determined with certainty which of those processes is more relevant to produce a higher index of neuronal mismatch in SRAF and PAF than in A1 (Fig. 5B), and therefore, we propose that it is caused by a moderate contribution from each process. Nevertheless, note how RS units in SRAF and PAF tend to have a higher index of predictionerror than $\mathrm{A} 1$, which would contribute to a higher index of neuronal mismatch.

Some studies have proposed that deviance detection and adaptation properties of cortical neurons may be differen depending on the layers where they are located (Bastos et a l., 2012; Heilbron and Chait, 2018). We compared the distributions of the indexes of neuronal mismatch, repetition suppression and prediction

median. C) The instantaneous firing rate (median $\pm 95 \%$ confidence interval) was significantly lower for RS than for FS units for the 25 th. 10 th $(* *<00.01)$, 5th and 1st significantly lower for RS than for FS units for the 25 th, 10th (** $\mathrm{p}<0.01)$, Sth and 1 s
percentiles $(* *)<0.001)$, but not for the 50 th percentile. (For interpretation of the percentiles ( $\cdots p<0.001)$, but not for the 50th percentile. (For interpretation of the this article.) 
A
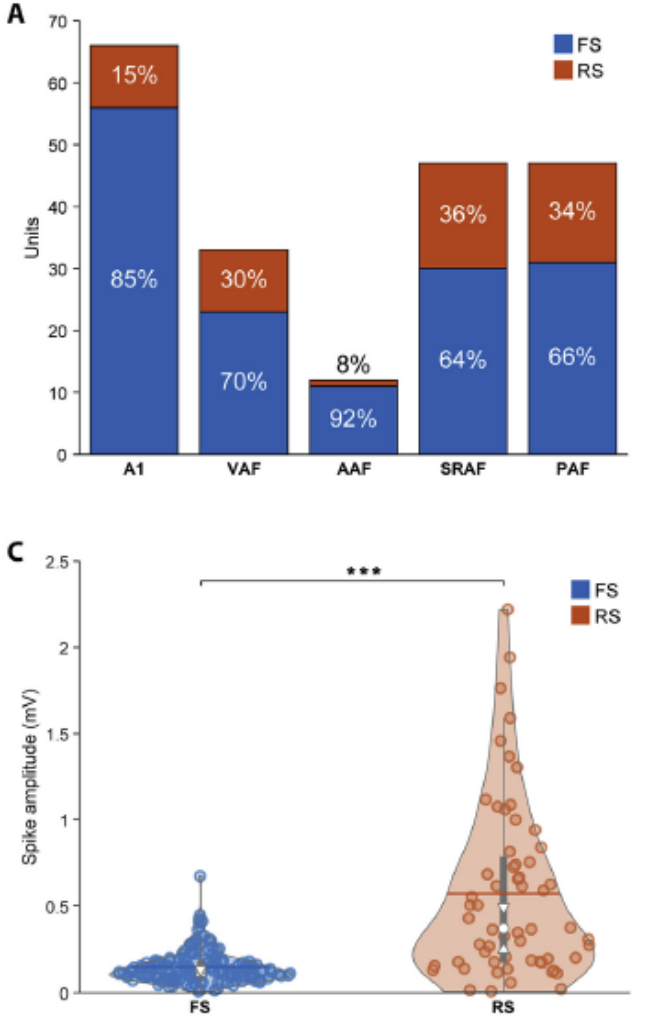
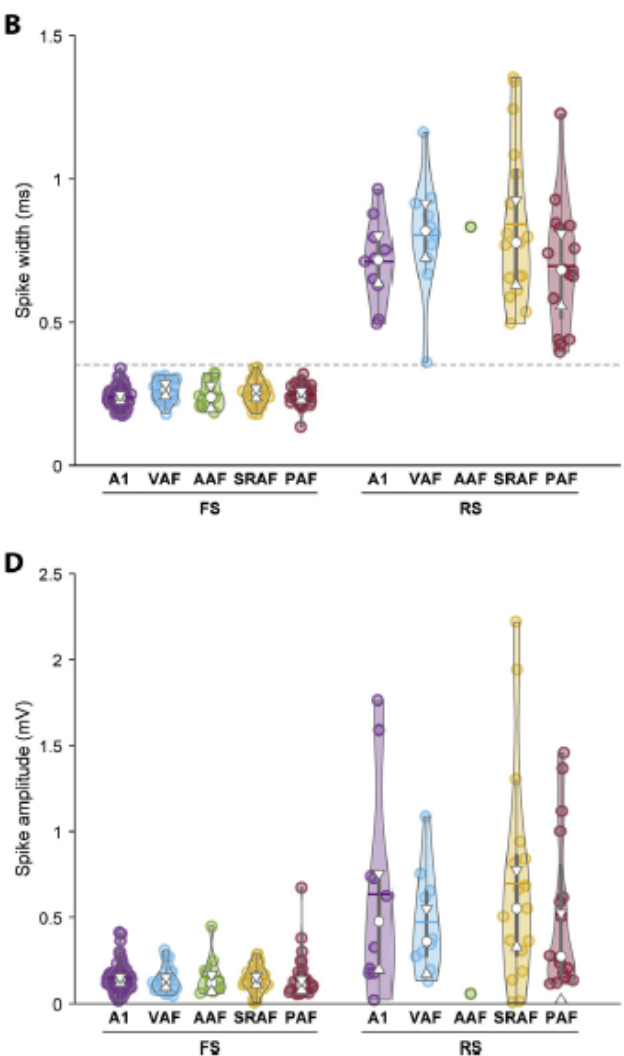

Fig. 3. Location of RS and FS units in AC cortical fields and spike amplitude. A) Number and percentage of RS (orange) and FS (blue) units bcated in the primary AC (A1), ventral Fig. 3. Location of RS and FS units in AC cortical fields and spike amplitude. A) Number and percentage of RS (orange) and FS (blue) units bocated in the primary AC (A1), ventral
auditory field (VAF), anterior auditory field (AAF), suprarhinal auditory field (SRAF) and posterior auditory field (PAF). B) Distribution of spike widths in RS and FS units for each auditory field. There were no significant differences in the distribution of spike widths across fields for neither RS nor FS units. C) Distribution spike amplitudes for RS and FS units The amplitude of RS units was significantly larger than that of FS units $(\cdots * \mathrm{p}<0.001)$. D) Distribution of spike amplitudes in RS and $\mathrm{FS}$ units for each auditory field. There were no significant differences in the distribution of spike amplitudes across fields for neither RS nor FS units. (For interpretation of the references to colour in this figure legend, the reader
is referred to the Web version of this article.)

error between supragranular, granular and infragranular layers, but we did not find any statistical differences, neither for all units together (Kruskal-Wallis, $\chi^{2}(2, N=202)=2.83, p=0.243 ; \chi^{2}(2$ $N=202)=1.25, p=0.535 ; \chi^{2}(2, N=202)=4.23, p=0.121$; respectively for each index) nor when considering RS (KruskalWallis, $\chi^{2}(2, N=54)=0.19, p=0.910 ; \chi^{2}(2, N=54)=2.00$ $p=0.367 ; \chi^{2}(2, N=54)=0.60, p=0.742$; respectively) or FS units separately (Kruskal-Wallis, $\chi^{2}(2, N=148)=3.77, p=0.152 ; \chi^{2}(2$, $N=148)=0.17, p=0.919 ; \chi^{2}(2, N=148)=4.50, p=0.105$; respectively).

\section{Discussion}

In the present study we compared the deviance detection properties on putative excitatory and inhibitory single units recorded from the AC of anaesthetized rats. Putative inhibitory units had narrower spikes, increased firing rates and smaller spike amplitudes relative to putative excitatory units. While putative inhibitory units spread through all cortical layers, putative excitatory units were mainly confined around layers III-V. Both putative inhibitory and excitatory units showed deviance detection properties, and in both cases the prediction error component was more relevant than repetition suppression.

The AC contains excitatory pyramidal neurons (glutamatergic) and inhibitory interneurons (GABAergic). Excitatory cells comprise $\sim 75 \%$ of the neural population and are highly correlated with pyramidal cell morphology (DeFelipe et al.,2013; Douglas and Martin, 2004; Prieto et al., 1994). In the present study, we found a much larger percentage of putative inhibitory than excitatory neurons (74 vs $26 \%$, respectively). This apparent sampling bias does not seem to be related to the cell size (putative excitatory neurons show larger spike amplitudes and SNRs), although the custom-made, high impedance tungsten electrodes that we use may allow us to record rather easily from smaller sized neurons. It is also unlikely to be an effect of the urethane anaesthesia; previous studies have shown that urethane affects both excitatory and inhibitory currents in a non-selective manner (Hara and Harris, 2002), and it does not alter excitatory glutamate- nor GABA-mediated synaptic transmission (Sceniak and Maclver, 2006)

Contrary to excitatory pyramidal neurons, there is a diversity of 
A

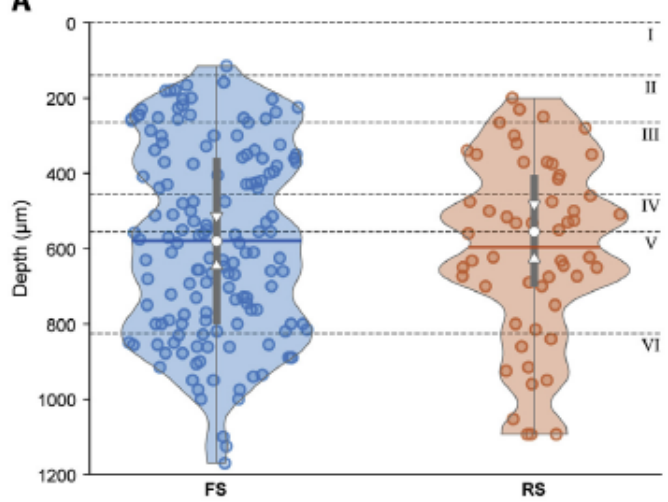

B

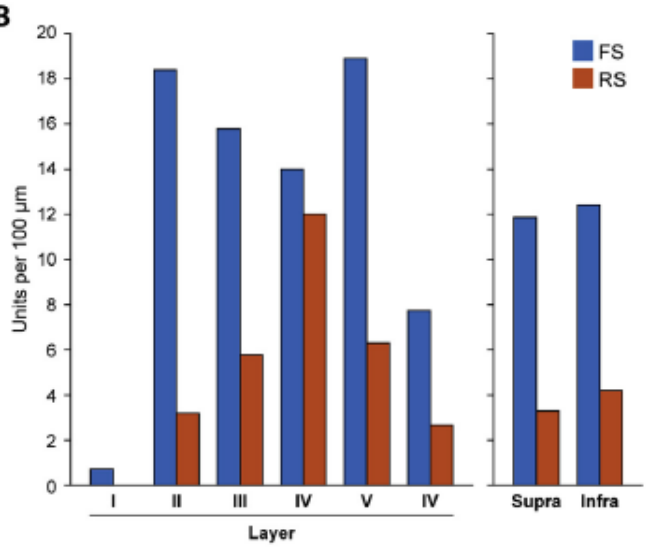

C

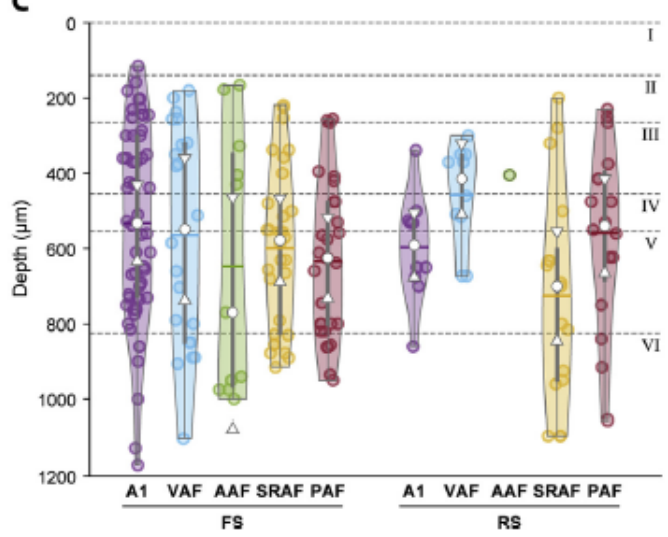

Fig. 4. Location of RS and FS units in cortical layers. A) Depth of the recording sites for $\mathrm{RS}$ and $\mathrm{FS}$ units. B) Left side: density of RS and $\mathrm{F}$ units per layer, calculated as number of units per $100 \mu \mathrm{m}(\mathrm{n}=202)$. RS units were most commonly found in layers $\mathrm{N}-\mathrm{V}$. while $\mathrm{FS}$ units spread over layers II-V. Right side: density of RS and $\mathrm{FS}$ units in supragranular (I-III) and inf ragranular (V-IV) layers. C) Distribution of recording site depths of RS and FS units for each auditory field. There were no significant differences inhibitory interneurons, which are usually classified using molecular markers. Inhibitory interneurons seem much more diverse because they may be classified according to different morphological, physiological, molecular, and synaptic properties (e.g., see Ascoli et al., 2008). It is rather common to classify cortical in terneurons according to the molecular markers they express, such as the calcium binding protein parvalbumin (PV, which includes chandelier and basket cells), the peptide somatostatin (SOM, such as Martinotti cells) or vasoactive intestinal peptide (VIP, as horsetail cells). These types comprise the majority of cortical interneurons, although they can be further subdivided using other markers (DeFelipe et al., 2013), and there is also evidence for functionally distinct SOM interneurons (Muñoz et al., 2017). Also, some authors propose that VIP interneurons are a subtype of a larger group which would be characterized by the expression of the ionotropic serotonin receptor 5HT3a (Rudy et al., 2011). VIP neurons inhibit SOM and PV interneurons, and thus participate in disinhibitory cortical microcircuit ( $\mathrm{Pi}$ et al., 2013).

After the seminal study from Mountcastle et al. (1969) that proposed that, in general terms, inhibitory and excitatory cortica neurons could be distinguished by the shape of their spikes, other studies have found similar results, adding evidence in support of this type of classification (e.g., Hefti and Smith, 2000; Kawaguchi and Kondo, 2002; Moore and Wehr, 2013). This classification has proven very useful, since it adds relevant information about the function of the units (which is not available using conventional recordings) and can be applied directly to most extracellular recordings (without the need for additional, more complex techniques). It has been successfully used in multiple studies, although with some methodological variations, such as in the references used for measuring the spike widths (e.g., time from peak to trough: Atencio and Schreiner, 2008; half-width, i.e. full width at half-maximal amplitude: Chen et al., 2015; half-valley width: Inse and Barnes, 2015; valley to peak width and half-peak width: Mendoza et al., 2016; Peyrache et al., 2012; width of "after-hyperpolarization": Andermann et al., 2004) or the aid of additional dimensions (firing rate, spike amplitude, peak asymmetry, etc) to refine the classification. Previous studies using a spike width measurement methodology most similar to ours (Barthó et al. 2004; Insel and Barnes, 2015; Mendoza et al., 2016), found that the best spike width cutoff for separating FS and RS would range between $\sim 0.25$ and $0.4 \mathrm{~ms}$ (if not considering the other dimensions measured), which is analogous to the $0.35 \mathrm{~ms}$ cutoff used here.

The general characteristics of FS and RS units after our classification are in agreement with previous reports. Apart from narrower spike widths, FS units showed increased firing rates and smaller spike amplitudes relative to RS units (Andermann et at. 2004: Atencio and Schreiner, 2008; Baeg et al, 2001; Bruno and Simons, 2002; Calabrese and Woolley, 2015; Chen et al., 2015; Hefti and Smith, 2000; Insel and Barnes, 2015; Mendoza et al. 2016; Moore and Wehr, 2013; Mountcastle et al. 1969; Peyrache et al., 2012). The distribution of FS and RS units across cortica layers differed in some degree from the actual locations of pyramidal neurons and intemeurons reported using histologica methods (Games and Winer, 1988). While putative inhibitory units were found across all cortical layers (with increased densities in layers II-V) as expected, putative excitatory units were found mainly in layers III-V, peaking at layer IV (Fig. 4B); however, according to histology, pyramidal neurons are very scarce in layer IV. Since the measurement of the recording depth in the present study was based on indirect methods (depth of the recording site as

in the distribution of spike amplitudes across fields for FS units nor for RS units. Approximate cortical layer depths (I-VI) based on Games and Winer (1988)

Please cite this article as: Pérez-González, D et al., Deviance detection in physiologically identified cell types in the rat auditory cortex, Hearing Research, https://doi.org/10.1016/j.heares.2020.107997 

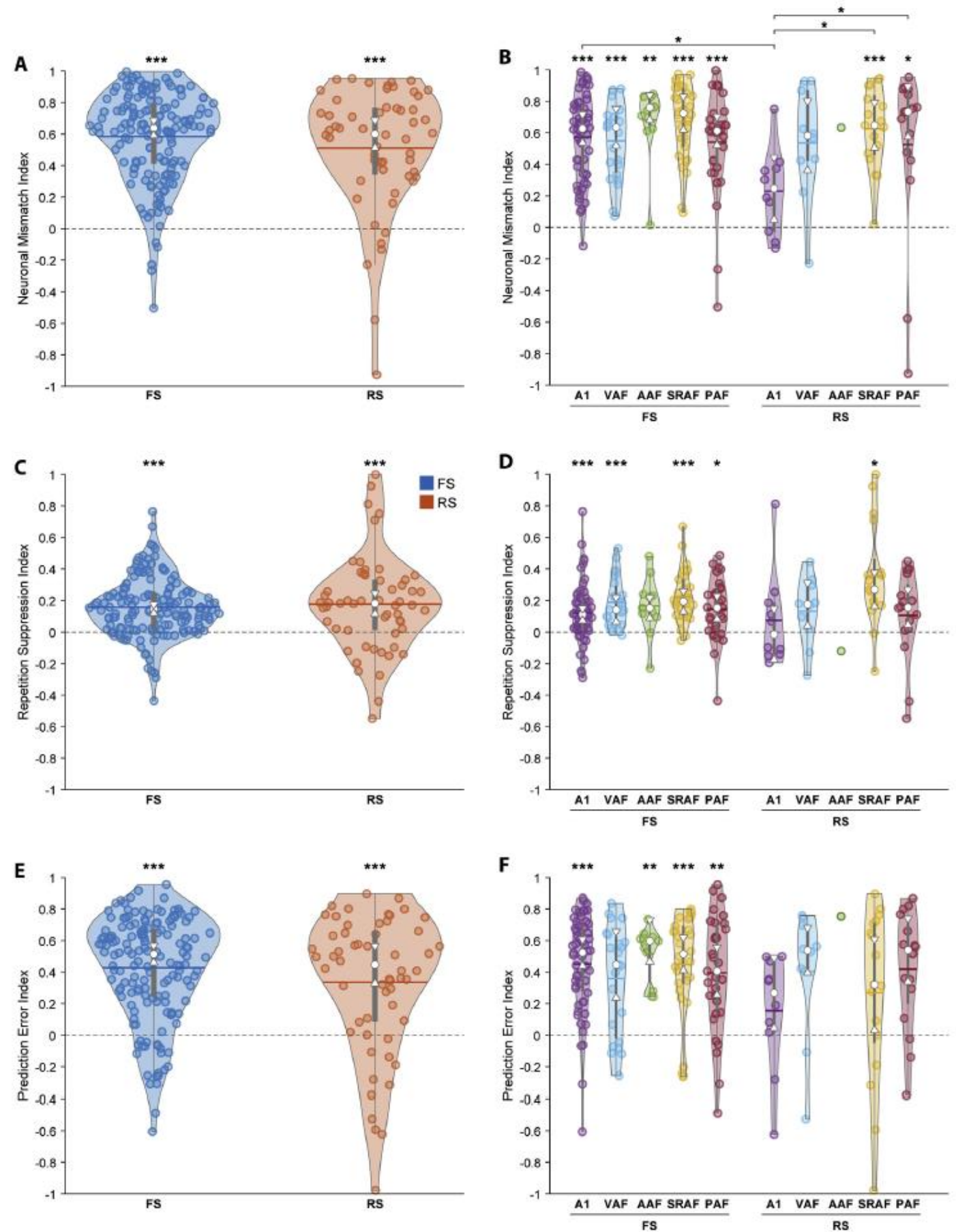

Fig. 5. Indexes of Neuronal Mismatch Repetition Suppression and Prediction Error.A,B) Distribution of the index of neuronal mismatch of RS and $\mathrm{FS}$ units for each auditory fiedd. C.D) Distribution of the index of repection suppression of RS and FS units for each auditory field. E.F) Distribution of the index of prediction error of RS and $\mathrm{FS}$ units for each auditory field Asterisks above the violin plots indicate that the group average is different from zero: brackets indicate differences between groups $\left({ }^{*} \mathrm{p}<0.05, * \mathrm{p}_{0}<0.01, \cdots * \mathrm{p}<0.001\right)$.

Please cite this article as: Pérez-González, D et al., Deviance detection in physiologically identified cell types in the rat auditory cortex, Hearing Research, https://doi.org/10.1016/j.heares.2020.107997 
indicated on the microdrive), it is possible that the units estimated to be near the borders between layers could be actually located in the adjacent layer. Considering that layer IV spreads only about $100 \mu \mathrm{m}$, a portion of neurons assigned to layer IV could easily be located in layers III or V.

The role of inhibition in the generation of deviance detection responses is still unclear. In subcortical nuclei, it has been shown that GABAergic inhibition has a modulatory effect, acting as a gain control mechanism that enhances and sharpens SSA (Duque et al., 2014; Pérez-González et al., 2012; Pérez-González and Malmierca, 2012). Many studies have found a dependence of MMN on Nmethyl-d-aspartate (NMDA) glutamate receptors, which would suggest a relevant role of excitatory cortical neurons in the generation of deviance detection, but we should not ignore the modulatory effect of inhibition on these responses (for a review, see Askew and Metherate, 2016). We found that both FS and RS units in the $A C$ showed similarly high levels of deviance detection (measured as the index of neuronal mismatch, Fig. 5A and B). This finding conforms with previous studies that found that both excitatory and inhibitory (PV and SOM) neurons in the AC exhibit SSA (Chen et al., 2015; Natan et al., 2015). Using whole cell recordings, Chen et al. (2015) found a long latency (mainly subthreshold) component in excitatory neurons, reflecting genuine deviance detection. Interestingly, Natan et al. (2015) found that both types of interneurons contribute to increased SSA in excitatory neurons, but through different mechanisms: optogenetic suppression of SOM interneurons increased the response of excitatory neurons to the standard tones but not to the deviant tones, while suppression of PV interneurons produced an equal increase in the response of excitatory neurons to both standard and deviant tones. The potentially different roles of the various types of inhibitory cortical interneurons are paramount for defining the circuits involved in deviance detection; unfortunately, the methodology of the present study does not allow for the differentiation of subtypes of inhibitory neurons, but it clearly demonstrates that GABAergic neurons play indeed a key role in SSA and deviance detection generation.

The deviance detection properties of both FS and RS units were the result of a high contribution of the prediction error component and reduced repetition suppression. We found that both types of units show similar deviance detection capabilities, as shown by the distributions of index of neuronal mismatch (Fig. 5A), and also in both cases the prediction error component (Fig. 5E) has a stronger weight on deviance detection than the repetition suppression component (Fig. 5C). But when looking individually at each field, FS units tended to have average index values different from zero, which was not so common for RS units (Fig. 5B, D, F). Likewise, the distributions of RS and FS units for each field were not different, except for A1 (see below). However, we found some differences in the index of neuronal mismatch across AC fields, but only for RS units (Fig. 5B). In th is case, the putative excitatory units in A1 (a lemniscal field) showed smaller index of neuronal mismatch values compared to units in SRAF and PAF (both of them non-lemniscal fields). This is probably because RS units in SRAF and PAF tend to show larger index of prediction error values than RS units in A1, although this trend does not amount to a statistically significant difference. It is noteworthy that $\mathrm{A} 1$ was the only $\mathrm{AC}$ field where the index of neuronal mismatch was different for RS and FS units (Fig. 5B); FS units showed a larger index of neuronal mismatch than RS units, maybe due to larger index of prediction error. As a lemniscal field, A1 tends to show reduced deviance detection properties compared to non-lemniscal fields (Parras et al., 2017). While FS units from all AC fields have an index of neuronal mismatch statistically larger than zero, there is a clear distinction between lemniscal (A1, VAF and AAF) and non-lemniscal fields (SRAF, PAF) for RS units. In this group, only non-lemniscal fields have an index of neuronal mismatch significantly than zero. These findings suggest that in lemnisca fields mostly FS units contribute to deviance detection, while in non-lemniscal fields both FS and RS units could contribute to the larger deviance detection values found on those areas.

\subsection{Limitations}

The classification of cortical neurons based on the shape of their spikes is a convenient method that can be implemented on most laboratories performing extracellular recordings; it can even be applied to data retrospectively, as long as they have been stored with enough level of detail. However, this method is indirect, and thus it has some shortcomings. The main limitation may be amount of correspondence between spike shape and neuronal type. Originally, Mountcastle et al. (1969) assigned regular and thin spikes to pyramidal and non-pyramidal cells, respectively. Later studies based both on morphology and cytochemistry have defined a wealth of cortical cell types and subtypes; for instance, Winer (2011) defines 16 different cell types in the auditory cortex. Apart from the pyramidal neurons, also the spiny stellate cells from the granular layers are excitatory. While most inhibitory interneurons are non-pyramidal, there are some exceptions (Budinger and Kanold, 2018; DeFelipe et al., 2013). So, any classification of excitatory/inhibitory neurons based on spike width (alone or accompanied by other measurements) is likely misclassifying a portion of the units.

It can be difficult to estimate how large the deviance detection indexes need to be in order to be functionally significant. As a reference, we can compare the index values found in lemniscal and non-lemniscal AC fields, which are notorious for having different deviance detection capabilities. A previous study, using methods comparable to those used here, found that the median index of neuronal mismatch only differed 0.10 between lemniscal and nonlemniscal AC fields (0.50 vs 0.60, Parras et al., 2017). Hence, relatively small differences in this index may imply substantial physiological distinctions. In the present study, the difference in the median values of the index of neuronal mismatch between RS and FS units is only 0.04 ( 0.60 vs 0.64 , respectively, Fig. $5 \mathrm{~A})$, maybe too small to be functionally relevant. On the other hand, if we look into A1, the difference reaches 0.38 ( 0.25 vs 0.63 , Fig. 5B). This suggests that taking all $\mathrm{AC}$ units as a whole, the deviance detection sensitivity of RS and FS units appears to be very similar, whereas in some particular areas the differences could be quite substantial.

The use of modern techniques, such as optogenetics or twophoton microscopy, allowing the selective activation/inactivation or the labelling of known cell types while recording from those neurons, may decrease the current uncertainties of using this classification.

\section{Conclusions}

Measurements of spike widths in extracellular recordings from the anesthetized rat AC can be used to classify the units as putative excitatory or inhibitory. This classification adds a layer of information to the neuronal activity recorded, which is unavailable using basic extracellular electrophysiology methods. In a set of recordings obtained in response to an auditory oddball paradigm, we found that both putative excitatory and inhibitory neurons in the rat $\mathrm{AC}$ show similar levels of deviance detection. When comparing the responses to the oddball paradigm to the responses to the corresponding control sequences, and interpreting the results under the predictive coding framework, we found that the responses of both types of neurons reflect a predominance of the prediction error signalling rather than repetition suppression. 
Altogether, the results obtained in this study suggest the involvement of both types of neurons in the circuits that generate deviance detection.

\section{CRediT authorship contribution statement}

David Pérez-González: Conceptualization, Methodology, Software, Formal analysis, Visualization, Writing - original draft, Writing - review \& editing. Gloria G. Parras: Investigation, Data curation. Camilo J. Morado-Díaz: Investigation, Data curation. Cristian Aedo-Sánchez: Investigation, Data curation. Guillermo V. Carbajal: Investigation, Data curation. Manuel S. Malmierca: Conceptualization, Writing - review \& editing, Supervision, Project administration, Funding acquisition.

\section{Acknowledgements}

This work was supported by a Spanish MINECO Grant (SAF201675803-P) to MSM and a Juan de la Cierva Fellowship (FJCl-201627897) to CJMD. We would like to thank Dr Javier Nieto-Diego for his support in the development of some of the analytical tools.

\section{Appendix A. Supplementary data}

Supplementary data to this article can be found online at https://doi.org/10.1016/j.heares.2020.107997.

\section{References}

Andermann, M.L., Ritt, J., Neimark, M.A., Moore, C.., 2004. Neural correlates of vibrissa resonance: band-pass and somatotopic representation of highfrequency stimuli. Neuron 42, 451-463. https://doi.org/10.1016/S0896frequency stimul

Anderson, LA., Malmierca, M.S., 2013. The effect of auditory cortex deactivation on stimulus-specific adaptation in the inferior collialus of the rat. Eur I Neurose 37, 52-62. https://doi.org/10.1111/ejn.12018. Antunes, F.M.. Malmierca, M.S., 2014. An overview of stimulus-specific adaptation
in the auditory thalamus. Brain Topogr. 27, 480-499. https://doi.org/10.1007 s10548-013-0342-6.

Antunes, F.M., Nelken, L., Covey, E, Malmierca, M.S., 2010. Stimulus-specific adaptation in the auditory thalamus of the anesthetized rat. PloS One 5, e14071. https://doiorg/10.1371/journal.pone.0014071.

Ascoli, G.A., Alonso-Nandares, L., And Piccione, R., Burkhalter, A, Buzsáki, G., Cauli, B., Defelipe, J., Faiŕńn, A Feldmeyer, D., Fishell, G., Fregnac, Y., Freund, T.F., Gardner, D., Gardner, E.P. Goldberg, J.H., Helmstaedter, M., Hestrin S., Karube, F. Kisvárday, ZF Lambolez, B. Lewis, D.A., Marin, O., Markram, H., Mũoz, A. Packer, A Petersen C.C.H., Rockland, K., Rossier, J., Rudy, B., Somogyi. P., Staiger, J.F. Tamas, G. Thomson, A.M., Toledo-Rodriguez, M., Wang, Y., West, D.C., Yuste, R. 2008. Petilla terminology: nomenclature of features of GABAergic interneuron of the cerebral cortex. Nat. Rev. Neurosd. 9, 557-568. https://doiorg/10.1038 $\operatorname{lin} 2402$

Askew, C.E, Metherate, R., 2016. Synaptic interactions and inhibitory regulation in auditory cortex Biol Psychol. 116, 4-9. https://doiorg/10.1016

Atencio, CA, Schreiner, C.E., 2008. Spectrotemporal processing differences between auditory cortical fast-spiking and regular-spiking neurons. J. Neurosci. 28 (2.

政 detection in the inferior colliculus. Front. Neural Circ. 6, 89. https://doi.org $0.3389 /$ fndir 2012.00089

Ayala, YA., Pérez-González, D., Duque, D., Nelken, L., Malmierca, M.S., 2013. Frequency discrimination and stimulus deviance in the inferior colliculus and cochlear nudeus. Front Neural Girc 6, 119. hitpst/doiorg/103389/ fncir.2012.00119.

Ayala, YA., Pérez-González, D., Malmierca, M.S., 2016. Stimulus-specific adaptation in the inferior colliculus: the role of excitatory, inhibitory and modulatory in-
puts. Biol. Psychol 116, 10-22. https://doi.org/10.1016/j.biopsycho.2015.06.016.

Ayala, Y.A. Udeh A., Dutta, K., Bishop, D., Malmierca, M.S., Oliver, D.L., 2015. Differences in the strength of cortical and brainstem inputs to SSA and non-SSA neurons in the inferior colliculus. Sa. Rep. 5, 1-17, https://doiorg/10.1038 srep 10383 .

Baeg, E.H., Kim, B.K.. Jang, J., Kim, H.T., Mook-Jung, L., Jung, M.W., 200 L. Fast spiking and regular spiking neural correlates of fear conditioning in the medial prefrontal cortex of the rat. Cerebr. Cortex 11, 441-451. https://doiorg/10.1093/ cercor/11.5.441.
Barthó, P., Hirase, H., Monconduit, L., Zugaro, M.. Harris, K.D., Buzsáki, G., 2004. Characterization of neocortical principal cells and interneurons by network interactions and extracellular features. J. Neurophysiol 92,600-608. https:/1 doi.org/10.1152/jn.01170.2003.

Bastos, A.M., Usrey, W.M., Adams, R.A, Mangun, G.R., Fries, P., Friston, K.J., 2012. Canonical micoodrcuits for predictive coding. Neuron 76, 695-711. https:// doi.org/10.1016/j.neuron.2012.10.038.

Bruno, R.M., Simons, D.J., 2002. Feedforward mechanisms of excitatory and inhibitory cortical receptive fields. J. Neurosd. 22, 10966-10975.

Budinger, E. Kanold, P.O., 2018. Auditory cortex dircuits. In: Oliver, D.L. Cant, N.B. Fay, R.R., Popper, A.N. (Eds.), The Mammalian Auditory Pathways: Synaptic Organization and Microcircuits. Springer. pp. 199-233. https://doi.org/10.1007] 978-3-319-71798-2_8,

Calabrese, A., Woolley, S.M.N., 2015. Coding principles of the canonical cortical microcircuit in the avian brain. Proc. Natl. Acad. Sci 112, 3517-3522. https:// doi.org/10.1073/pnas.1408545112.

Carbajal, G.V., Malmierca, M.S., 2018. The neuronal basis of predictive coding along the auditory pathway: from the subcortical roots to cortical deviance detection. Trends Hear 22. https://doi.org/10.1177/2331216518784822, 23312 1651878482.

Casado-Román, L., Pérez-González, D., Malmierca, M.S., 2019. Prediction errors explain mismatch signals of neurons in the medial prefrontal cortex. bioRxiv 778928. https://doi.org/10.1101/778928.

Chen, LW., Helmchen, E., Lūtcke, H., 2015. Specific early and late oddball-evoked responses in excitatory and inhibitory neurons of mouse auditory cortex. J. Neurosci. 35, 12560-12573. https://doi.org/10.1523/JNEUROSCI.2240-15.2015. Defelipe, J., López-Cruz, P.L., Benavides-Piccione, R., Bielza, C., Larrañaga, P., Anderson, S., Burkhalter, A, Cauli, B., Fairen, A, Feldmeyer, D., Fishell, G., Fitzpatrick, D., Freund, T.F., Gonzalez-Burgos, G., Hestrin, S., Hill, S., Hof, P.R., Huang, J., Jones, E.,., Kawaguchi, Y., Kisvarday, Z., Kubota, Y., Lewis, DA., Rockland $K_{-}$Rossier, J Rulens Rockland, K., Rossier, J., Rubenstein, J.L.R., Rudy, B., Scanziant, M., Yuste, $R$, Ascoli, GA, 2013. New insights into the classification and noment dature of corticat GaBAergic interneurons. Nat. Rev. Neurosdi. 14, $202-216$ hature of cortical GABAergic int

Douglas, R.J. Martin, K.A.C., 2004. Neuronal circuits of the neocortex. Annu. Rev. Neurosi. 27.245 . $419-451.21004$ https://doi.org/10.1146/

Duque, D., Malmierca, M.S., Caspary, D.M., 2014. Modulation of stimulus-specific adaptation by GABAA receptor activation or blockade in the medial geniculate body of the anaesthetized rat. J. Physiol. 592, 729-743. https://doi.org/10.1113/ jphysiol.2013.261941.

Duque, D., Perez-Gonzalez, D., Ayala, YA., Palmer, A.R., Malmierca, M.S., 2012. Topographic distribution, frequency, and intensity dependence of stimulusspecific adaptation in the inferior colliculus of the rat. J. Neurosci. 32, 17762-17774. https://doi.org/10.1523/JNEUROSCl.3190-12.2012.

Friston, K., 2005. A theory of cortical responses. Philos. Trans. R. Soc. B Biol. Sci 360, 815-836. https://doi.org/10.1098/rstb.2005.1622.

Games, K.D., Winer, J.A, 1988. Layer V in rat auditory cortex: projections to the inferior colliculus and contralateral cortex. Hear. Res. 34, 1-25. https://doi.org/ $10.1016 / 0378-5955(88) 90047-0$.

Garrido, M.L., Kilner, J.M., Stephan, K.E., Friston, KJ.. 2009. The mismatch negativity: a review of underlying mechanisms. Clin. Neurophysiol. 120, 453-463. https: // doi.org/10.1016/j.clinph.2008.11.029

Hara, K. Harris, RA, 2002. The anesthetic mechanism of urethane: the effects on neurotransmitter-gated ion channels. Anesth. Analg. 94, 313-318.

Hefti, B.J., Smith. P.H., 2000. Anatomy, physiology, and synaptic responses of rat layer V auditory cortical cells and effects of intracellular GABA(A) blockade. J. Neurophysiol. 83, 2626-2638. https://doi.org/10.1152/jn.2000.83.5.2626.

Heilbron, M., Chait, M., 2018. Great expectations: is the re evidence for predictive
coding in auditory cortex? Neuroscience 389, 54-73. https://doiorg/10.1016/ coding in auditory cortex

Insel, N., Barnes, C.A, 2015. Differential activation of fast-spiking and regular-firing neuron populations during movement and reward in the dorsal medial frontal cortex. Cerebr. Cortex 25, 2631-2647. https://doi.org/10.1093/cercor/bhu062.

Kawaguchi, Y., Kondo, S., 2002. Parvalbumin somatostatin and cholecystokinin as chemical markers for specific GABAergic interneuron types in the rat frontal cortex. J. Neurocytol. 31, 277-287. https://doi.org/10.1023/A: 1024126110356.

Kostal, L., Lansky, P., Stiber, M., 2018. Statistics of inverse interspike intervals: the instantaneous firing rate revisited. Chaos $28,1-8$. https://doi.org/10.1063/ 1.5036831.

Malmierca, M.S., 2015. Auditory system. In: Paxinos, G. (Ed.), The Rat Nervous System. Elsevier - Academic Press, pp. 865-946.

Malmierca, M.S., Anderson, L.A. Antunes, F.M., 2015. The cortical modulation of stimulus-specific adaptation in the auditory midbrain and thalamus: a potential neuronal correlate for predictive coding. Front Syst. Neurosd. 9, 1-14. https: // doi.org/10.3389/fnsys.2015.00019

Malmierca, M.S., Cristaudo, S., Pérez-González. D., Covey, E., 2009. Stimulus-specific adaptation in the inferior colliculus of the anesthetized rat. J. Neurosci. 29, 5483-5493. https://doiorg/10.1523/JNEUROSC1.4153-08.2009.

Mendoza, G., Peyrache, A. Gámez, J., Prado, L., Buzsáki, G., Merchant, H., 2016. Recording extracellutar neural actwity in the behaving monkey using a semichronic and high-density electrode system. J. Neurophysiol. 116, 563-574. https://doiorg/10.1152/jn.00116.201

Michie, P.T., Malmierca, M.S., Harms, L., Todd, J., 2016a. The neurobiology of MMN 
and implications for schizophrenia. Biol. Psychol. 116, 90-97. https://doi.org/ 10.1016/j.biopsycho.2016.01.011.

Michie, P.T., Malmierca, M.S., Harms, L., Todd, J., 2016b. Understanding the neurobiology of MMN and its reduction in schizophrenia. Biol. Psychol. 116, 1-3. https://doi.org/10.1016/j.biopsycho.2016.02.005

Moore, AK. Wehr, M., 2013. Parvalbumin-expressing inhibitory interneurons in auditory cortex are well-tuned for frequency. J. Neurosci. 33, 13713-13723 https://doi.org/10.1523/JNEUROSC.0663-13.2013.

Mountcastle, V.B. Talbot, W.H., Sakata, H., Hyvärinen, J.. 1969. Cortical neuronal mechanisms in flutter-vibration studied in unanesthetized monkeys. Neuronal periodicity and frequency discrimination. J. Neurophysiol. 32, 452-484. https:/)

Muñoz, W, Tremblay R., Levenstein, D., Rudy, B., 2017. Layer-specific modulation of neocortical dendritic inhibition during active wakefulness. Science (80-. ) 355 954-959. https://doi.org/10.1126/science.aag2599.

Ngatinen R. Gailland, A.W Mantysalo, S. 1978 . Earty selective-attention effect on evoked potential reinterpreted. Acta Psychol. 42, 313-329.

Näătänen, R., Kujala, T., Escera, C., Baldeweg, T., Kreegipuu, K., Carlson, S., Ponton, C. 2012. The mismatch nega tivity (MMN) - a unique window to disturbed central auditory processing in ageing and different dinical conditions. Clin. Neuroauditory processing in age ing and different dinical conditions. Clin.
physiol. 123,424-458. https://doi.org/10.1016/j.clinph.2011.09.020.

Natan, R.G., Briguglio, J.J., Mwilambwe-Tshilobo, L., Jones, S.L., Aizenberg, M., Goldberg, E.M., Geffen, M.N., 2015. Complementary control of sensory adaptation by two Nieto-Diego,J., Malmierca, M.S., 2016. Topographic distribution of stimulus-specific
adaptation across auditory cortical fields in the anesthetized rat. PloS Biol. 14, 1-30. https://doi.org/10.1371/journal.pbio.1002397.

Pandya, P.K., Rathbun, D.L., Moucha, R., Engineer, N.D., Kilgard, M.P., 2008. Spectral and temporal processing in rat posterior auditory cortex. Cerebr. Cortex 18 301-314. https://doi.org/10.1093/cercor/bhm055

Parras, G.G., Nieto-Diego, J., Carbajal, G.V., Valdés-Baizabal, C., Escera, C Malmierca, M.S., 2017. Neurons along the auditory pathway exhibit a hierarchical organization of prediction erroc. Nat. Commun. 8 https://doi.org/10.1038/ \$41467-017-02038-6.

Parras, G.G., Valdés-Baizabal, C., Harms, L., Michie, P., Malmierca, M.S., 2019. The effect of NMDA-R antagonist, MK-801, on neuronal mismatch along the auditory thalamocortical pathway. bioRxiv 636068. hittps://doi.org/10.1101/636068.

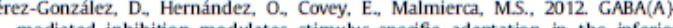
mediated inhibition modulates stimulus-specific adaptation in the inferio

Pérez-González, D., Malmierca, M.S., 2014. Adaptation in the auditory system: an overview. Front. Integr. Neurosci. 8, 19. https://doi.org/10.3389/
(n) fnint.2014.00019.

Pérez-González, D., Malmierca, M.S., 2012. Variability of the time course of stimulus-specific adaptation in the inferior colliculus. Front. Neural Girc. 6 2012.00107

Pérez-González, D., Malmierca, M.S., Covey, E., 2005. Novelty detector neurons in doi.org/10.1111/.1460-95682005.04472.

Peyrache, A., Dehghani, N., Eskandar, E.N., Madsen, J.R., Anderson W.
Donoghue, JA., Hochberg, LR., Halgren, E., Cash, S.S., Destexhe, A., 2012. Spatiotemporal dynamics of neocortical excitation and inhibition during human sleep. Proc. Natl. Acad Sci USA. 109, 1731-1736. https://doiorg/10.1073/

Pi, H.J., Hangya, B., Kvitsiani, D., Sanders, J.., Huang, Z.J., Kepecs, A. 2013. Cortical interneurons that spedalize in disinhibitory control. Nature 503, 521-524.

Polley, D.B., Read, H.L., Storace, DA., Merzenich, M.M., 2007. Multiparametric auditory receptive field organization across five cortical fields in the albino rat. J Noterson BA. Winer JA 1994 Morphology and spatia distiber

r. GABAergic neurons in cat primary auditory cortex (AI). J. Comp. Neurol. 344,

Profant 0 - Burianova J Syla J 2013. The response

Profant, O., Burianová, J., Syka, J., 2013. The response properties of neurons in doifferent fields of the auditory cortex in

Rudy, B., Fishell, G., Lee, S.H., Hjerling-Leffler, 1., 2011. Three groups of interneurons account for nearly $100 \%$ of neocortical GABAergic neurons. Dev. Neurobiol. 71 , 45-61. https://doi.org/10.1002/dneu.20853.

Ruhnau, P., Herrmann, B., Schröger, E., 2012. Finding the right control: the mismatch negativity under investigation. din. Neurophysiol. 123,507-512. https:// doi.org/10.1016/j.clinph.2011.07.035.

Sceniak, M.P., Maclver, M.B. 2006. Cellular actions of urethane on rat visual cortical neurons in vitro. J. Neurophysiol. 95, 3865-3874. https://doi.org/10.1152/ jn. 01196.2005.

Smith, P.H., Uhlrich, D.., Manning, K.A., Banks, M.1., 2012. Thalamocortical projections to rat auditory cortex from the ventral and dorsal divisions of the medial geniculate nudeus. J. Comp. Neurol. 520, 34-51. https://doi.org/10.1002/ cne.22682.

Tsolaki, AC., Kosmidou, V., Kompatsiaris, L., Yiannis), Papadaniil, C., Hadjileontiadis, L., Adam, A. Tsolaki, M., 2017. Brain source localization of MMN and P300 ERPs in mild cognitive impairment and Alzheimer's disease: a highdensity EEG approach. Neurobiol. Aging 55, 190-201. https://doi.org/10.1016/

Ulanovsky, N., Las, L., Nelken, L., 2003. Processing of low-probability sounds by cortical neurons. Nat. Neurosd. 6, 391-398. https://doi.org/10.1038/nn1032.

Vlaskamp, C., Oranje, B., Madsen, G.F., Mallegaard Jepsen, J.R., Durston, S., Cantio, C., order: mismatch negativity deficits. Autism Res. 10, 1857-1865. https://doi.org/ order: mismatch 10.1002 aur.1821

Wiens, S. Szychowska, M., Eklund, R., van Berlekom, E., 2019. Cascade and norepetition rules are comparable controls for the auditory frequency mismatch repetition rules are comparable controls for the auditory frequency mismatch
negativity in oddball tasks. Psychophysiology 56, 1-12. https://doi.org/10.1111/ psyp.13280.

Winer, JA. 2011. Neurochemical organization of the medial geniculate body and auditory cortex, In: Winer, JA, Schreiner, CE. (Eds.), The Auditory Cortex. Springer, New York, pp. 209-234.

Winer, J.A., Sally, S.L. Larue, D.T., Kelly, J.B., 1999. Origins of medial geniculate body Hear. Res. 130, 42-61. https://doi.org/10.1016/S0378-5955(98)00217-2. 
The Role of Cholinergic Neuromodulation in Forwarding Prediction Error in the Auditory Cortex

Tracking no: 26-05-2020-RA-eLife-59347

Impact statement: This study demonstrates that acetylcholine scales the precision of prediction error signaling and is mediated by muscarinic receptors, gating prediction errors to hierarchically higher processing levels.

Competing interests: No competing interests declared

Author contributions:

Cristian Aedo-Sánchez: Data curation; Formal analysis; Investigation; Visualzation; Writing - original draft David Pérez-González:

Conceptualzation; Data curation; Formal analysis; Validation; Visualization; Methodology; Writing - review and editing Manuel Malmierca:

Conceptualzation; Supervision; Funding acquisition; Visualzation; Writing - original draft; Project administration; Writing - review and editing

Funding:

Ministerio de Economá y Competitividad (MINECO): Manuel S. Malmierca, SAF2016-75803-P; Agencia Estatal de Investigación. Ministerio de Ciencia e Innovación.: Manuel S. Malmierca, PID2019-104570RB-I00 The funders had no role in study design, data collection and interpretation, or the decision to submit the work for publication.

Data Availability:

All data generated or analysed during this study are included in the manuscript and supporting files. Source data files have been provided for Figures $1,2,3,4,5$ and 6 .

N/A

\section{Ethics:}

Human Subjects: No Animal Subjects: Yes Ethics Statement: All methodological procedures were approved by the Bioethics Committee for Animal Care of the University of Salamanca (Permit Nuumber: USAL-ID-195), and performed in complance with the standards of the European Convention ETS 123, the European Union Directive 2010/63/EU and the Spanish Royal Decree 53/2013 for the use of animals in scientific research. Surgical anesthesia was induced and maintained with urethane $(1.5 \mathrm{~g} / \mathrm{kg}$, intraperitoneal), with supplementary doses $(0.5 \mathrm{~g} / \mathrm{kg}$, intraperitoneal) given as needed. Dexamethasone $(0.25 \mathrm{mg} / \mathrm{kg})$ and atropine sulfate $(0.1 \mathrm{mg} / \mathrm{kg})$ were administered at the beginning of the surgery to reduce brain edema and the viscosity of bronchial secretions, respectively. Every effort possible was made to minimize suffering. 
The Role of Cholinergic Neuromodulation in Forwarding Prediction Error in the Auditory Cortex

Cristian Aedo-Sánchez ${ }^{1,2}$, David Pérez-González ${ }^{1,2}$ \& Manuel S. Malmierca ${ }^{1,2,3^{*}}$

${ }^{1}$ Cognitive and Auditory Neuroscience Laboratory, Institute of Neuroscience of Castilla y León, Calle Pintor Fernando Gallego 1, 37007 Salamanca, Spain

${ }^{2}$ Institute for Biomedical Research of Salamanca (IBSAL), 37007 Salamanca, Spain.

3 Department of Biology and Pathology, Faculty of Medicine, Campus Miguel de Unamuno, University of Salamanca, 37007 Salamanca, Spain

* Corresponding and leading author: msm@usal.es 


\begin{abstract}
A fundamental property of sensory systems is their ability to detect novel stimuli in the environment. The auditory brain contains neurons that decrease their response to repetitive sounds but that increase their firing rate against novel or deviant stimuli; the difference between both responses is known as stimulus-specific adaptation (SSA) or neuronal mismatch. This study describes how acetylcholine (ACh) modulates SSA in the rat auditory cortex. SSA is best explained by the predictive coding framework and neuromodulators such as $\mathrm{ACh}$ are thought to increase the precision of prediction error signaling. Results show that SSA increased by $\sim 31 \%$ after the microiontophoretic injection of ACh. Importantly, ACh increased the neuronal firing rate in response to deviant tones only, and only the prediction error component was affected. Thus, we demonstrate that $\mathrm{ACh}$ increases the precision of prediction error signaling and is mediated by muscarinic receptors, gating prediction errors to hierarchically higher processing levels.
\end{abstract}




\section{INTRODUCTION}

Neuromodulation strongly impacts sensory processing by influencing neuronal excitability or synaptic processes in neuronal circuits (Hasselmo and Giocomo, 2006; Lucas-Meunier et al., 2003; Metherate, 2011; Muñoz and Rudy, 2014; Picciotto et al., 2012). Acetylcholine (ACh) is a widely distributed neuromodulator throughout the brain, including the auditory cortex (AC), and modulates different neurobiological processes such as attention, learning, memory, arousal, sleep and/or cognitive reinforcement (Batista-Brito et al., 2018; Dalley et al., 2004; Franklin and Frank, 2015). The main source of ACh to the AC is the basal forebrain (Chavez and Zaborszky, 2017; Mesulam, 2013; Zaborszky et al., 2008). In the auditory system, cholinergic modulation is known to alter frequency response areas generating changes across frequency tuning, decreasing the acoustic threshold at the characteristic frequency and changing the encoding of spectral representation of many auditory neurons (Ma and Suga, 2005; Metherate, 2011) Thus, ACh promotes neuronal and synaptic plasticity at different temporal scales (Kamke et al., 2005; Kilgard and Merzenich, 1998)

Here, we characterized the effect of ACh on stimulus specific adaptation (SSA), a type of neuronal adaptation found in the AC. SSA is elicited by oddball sequences, which consist of a pattern of repeating sounds (standards), interrupted by a low-probability and unexpected sound (deviant). The deviant usually differs in frequency, but could differ on any other physical dimension from the standard, or otherwise violate a pattern of regularity established by the standard (I.-W. Chen et al., 2015; Nieto-Diego and Malmierca, 2016; Parras et al., 2017b; Ulanovsky et al., 2003; Von Der Behrens et al., 2009) 
Thus, neurons that exhibit SSA adapt specifically to the standard stimulus but resume their firing when a deviant stimulus appears. SSA has been proposed to be a neuronal correlate of 'mismatch negativity' (MMN), an evoked potential obtained in human and animal electroencephalographic studies using the oddball paradigm (Nieto-Diego and Malmierca, 2016; Ulanovsky et al., 2003) . Because SSA is considered a form of shortterm plasticity (Ogawa and Oka, 2015) and ACh has been shown to play a role in this type of neural plasticity (Marshall et al., 2016; Moran et al., 2013; Parr and Friston, 2017; Vossel et al., 2012) it is plausible that ACh may be involved in the generation and/or modulation of SSA. Furthermore, it has been shown that ACh differentially modulates the neural response to the standard stimulus in units of the IC (Ayala and Malmierca, 2015).

Currently, MMN and SSA are best explained by the predictive coding theory (Friston, 2008, 2005). According to the predictive coding framework, higher-level cortical areas generate predictions about the environment that are sent in a top-down manner to lower hierarchical levels, to suppress the ascending neuronal activity evoked by sensory events that can be anticipated. However, when current predictions do not match the sensory inputs, then the lower levels will send forward bottom-up prediction errors to higher hierarchical levels (Friston and Kiebel, 2009). Computational models weigh prediction errors of sensory input by their precision, which is the inverse of sensory variance, as prediction errors can exist in varying levels of uncertainty (Parr and Friston, 2017). More importantly, in neurobiological terms, precision has been suggested to be mediated by synaptic gain modulation, likely by cholinergic neuromodulation (Moran et al., 2013). 
Furthermore, according to the predictive coding model there are two mechanisms underlying the MMN/SSA (Auksztulewicz and Friston, 2016; G. V Carbajal and Malmierca, 2018; Friston, 2008; Harms et al., 2020; Parras et al., 2017b) .First, SSA could reflect the repetition suppression (RS) of the response to the predictable stimuli (standards). But SSA could also reflect a process of prediction error (PE) which is an enhanced neural response that is elicited upon the violation of a prediction when an unexpected (deviant) stimulus is presented. Repetition suppression and prediction error are now distinguished using control sequences (Ruhnau et al., 2012), and there is now evidence in both humans and rodents that MMN/SSA receives contributions from both prediction error and repetition suppression at various levels of the auditory system (Ishishita et al., 2019; Parras et al., 2017b).

The main goal of the present study is to determine the role that ACh plays in the modulation of SSA as well as repetition suppression and prediction error in the rat AC, utilizing control sequences to separate these components unambiguously (Nieto-Diego and Malmierca, 2016; Parras et al., 2017b). We used microiontophoretic injections of $\mathrm{ACh}$, as well as nicotinic and muscarinic receptor antagonists to determine how ACh neurotransmission affects the responses of AC neurons that exhibit SSA, in addition to the role of ACh on prediction error and repetition suppression, in both primary and secondary AC areas.

\section{RESULTS}

To explore the influence of cholinergic modulation on SSA and prediction error, we recorded a total of 122 units in the AC before, during and after the microiontophoretic injection of acetylcholine $(n=99)$. We also applied two antagonist drugs: scopolamine $(n=13)$ and 
mecamylamine $(\mathrm{n}=10)$ to test if the effects observed with ACh were mediated by muscarinic or nicotinic receptors, respectively. Recording depths ranged 140-1080 $\mu \mathrm{m}$ including neurons from all layers, except layer I.

In order to allocate each recorded neuron to a specific field in the AC, we recorded the FRA and analyzed the topographical distribution of CF for each unit. Each recording was assigned to a dorsoventral and rostrocaudal coordinate system relative to bregma as in previous studies (Nieto-Diego and Malmierca, 2016; Parras et al., 2017b; Polley et al., 2007). This analysis allowed us to pool the data from all animals (Fig. 1A) and construct a synthetic map of the $\mathrm{CF}$ across the entire rat auditory cortex (Nieto-Diego and Malmierca, 2016; Parras et al., 2017b). Similar to these previous works, we found a high-frequency reversal zone between ventral auditory field (VAF, caudally) and anterior auditory field (AAF, rostrally), a low-frequency reversal zone between A1 and posterior auditory field (PAF, dorsocaudally), and a highfrequency reversal between VAF and suprarhinal auditory field (SRAF, ventrally). Thus, we could reliably define the lemniscal (A1, AAF, and VAF) and non-lemniscal (SRAF, PAF) auditory cortical fields as shown in Fig. 1B. 

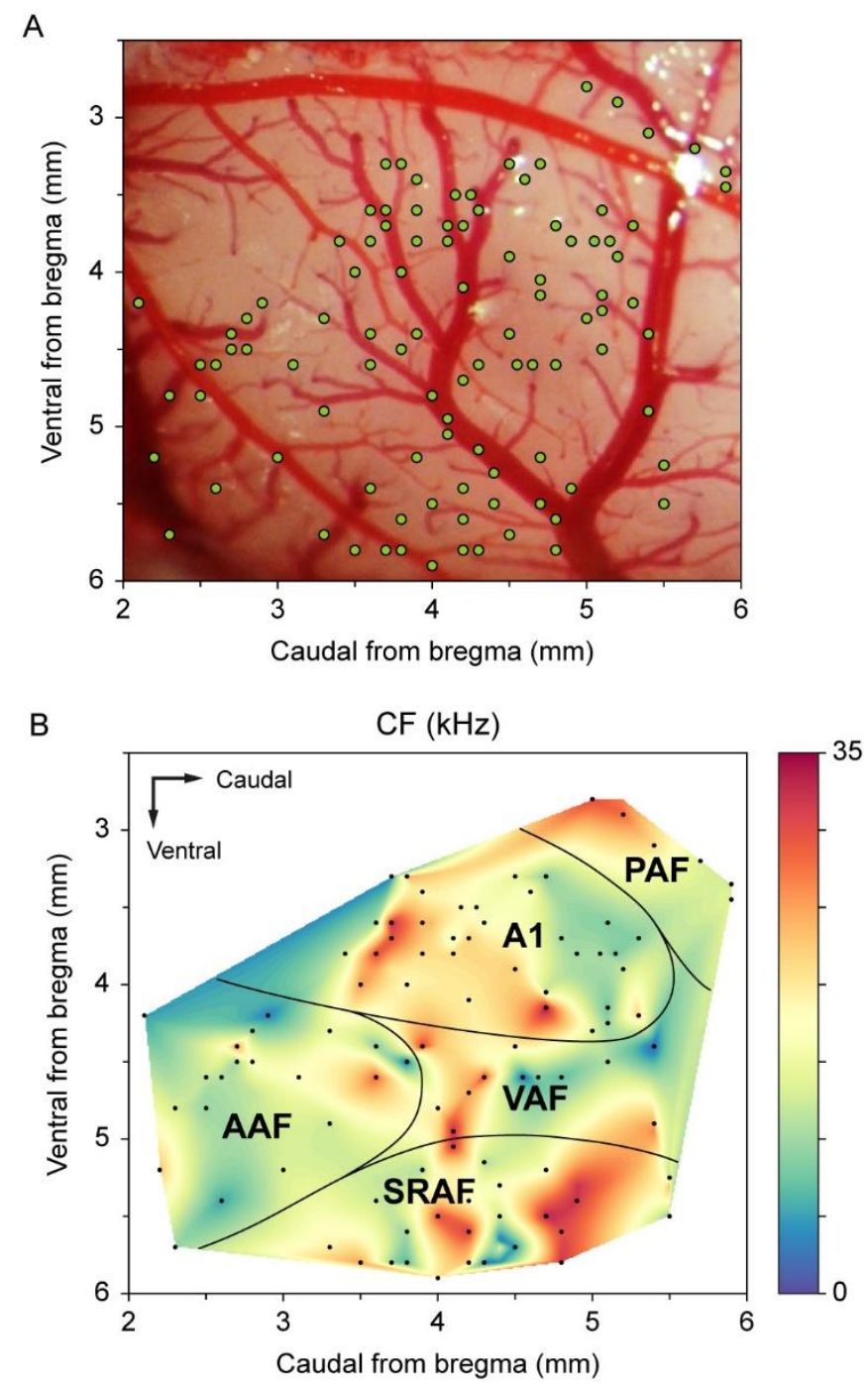

Figure 1: Map of all recording locations. (A) All recording sites drawn over the cortex of a representative animal. At every site, the CF was determined and then we presented an oddball paradigm and the corresponding control sequences. (B) Distribution of the CFs across the entire rat auditory cortex. Note how each field shows a characteristic CF gradient.

Figure 2 illustrates representative FRAs of five units from each of the auditory fields before, during and after ACh injection. As in these example cells, we observed an increase in the 
mean response after the ACh injections (mean control: $0.80 \pm 0.69$ spikes/stimulus; mean ACh: $1.62 \pm 1.12$ spikes/stimulus. Wilcoxon Signed Rank test, $\mathrm{p}<0.001)$. Most neurons recovered their basal firing rates after 60-90 minutes post ACh injection (recovery mean: 0.84 \pm 0.42 spikes/stimulus). After the FRA was measured, we selected a pair of pure tones (10-30 $\mathrm{dB}$ above minimum threshold) within the FRA at each recording site to test the adaptation sensitivity of the neuronal response under the oddball paradigm, to study SSA (Fig. 3). From here, we describe the effect of ACh, scopolamine and mecamylamine on SSA (Figs. 2-5) followed by the effect on prediction error and repetition suppression (Fig. 6).

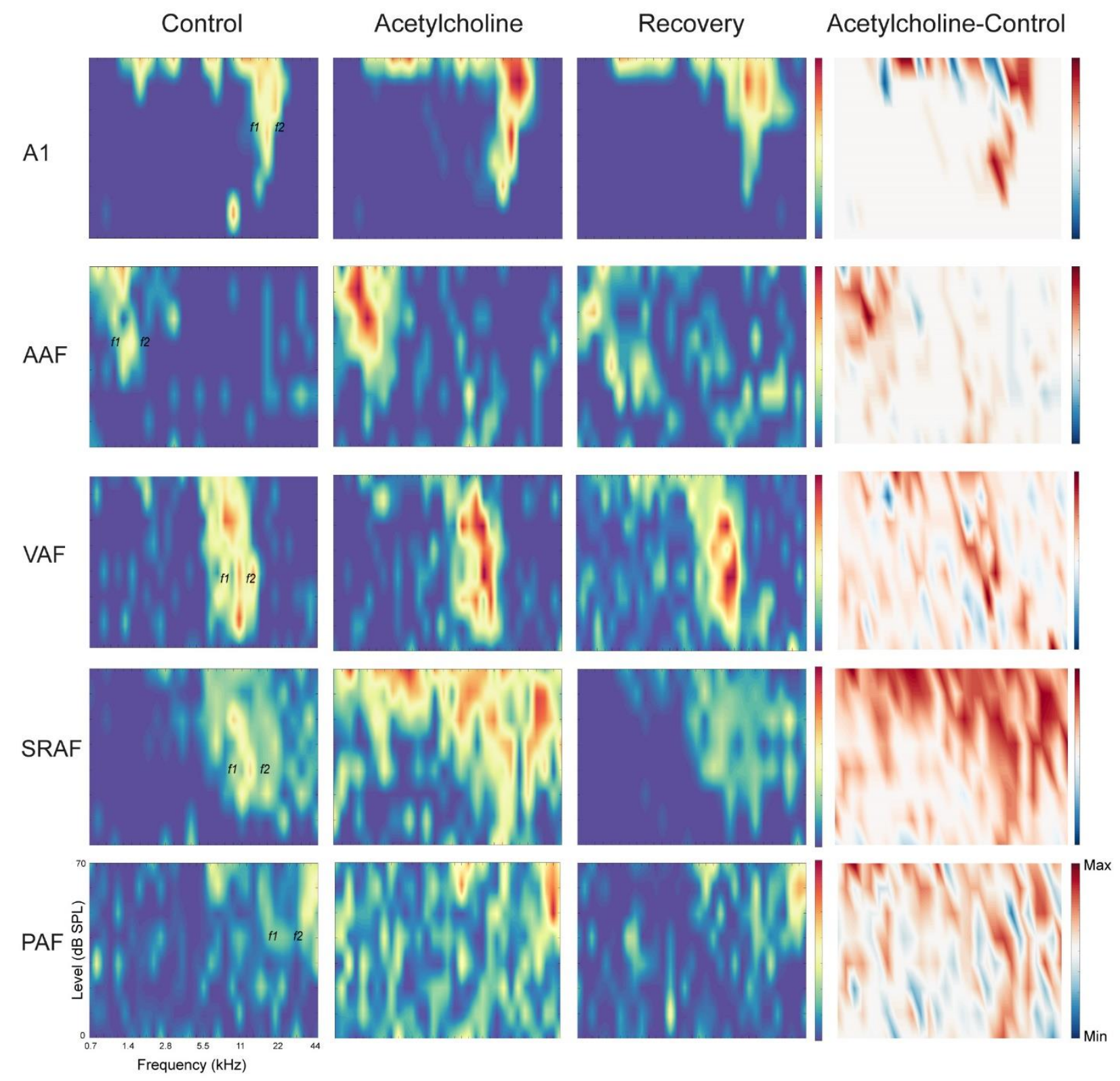


Figure 2: Examples of FRA changes in control, ACh and recovery conditions: Each row shows the FRA for a representative unit of each AC field (lemniscal: A1, AAF and VAF; and non-lemniscal: SRAF and PAF). In all the examples, the spike count increased after ACh injection ("Acetylcholine" column) and most of these neurons recovered their basal firing rates after 60-90 minutes post ACh injection ("Recovery" column). The "Acetylcholine-Control" column shows the difference in firing rate between ACh and control conditions. $f 1$ and $f 2$ indicate the location of the frequencies used for the oddball sequence on each unit. 

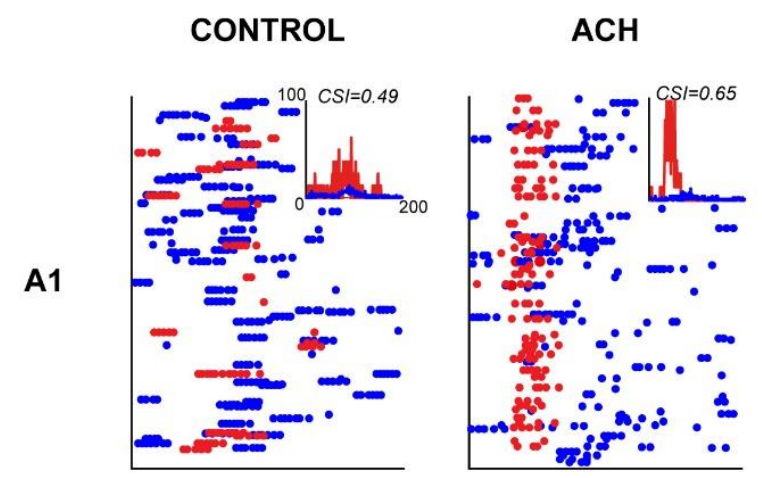

RECOVERY

Figure 3: Examples of neuronal responses in control, ACh and recovery conditions. The figure shows dot rasters and peri-stimulus time histograms (PSTH, insets) of five representative units (rows) in control (left column), ACh (central column) and recovery conditions (right column) from lemniscal (A1, AAF and VAF) and non-lemniscal (SRAF and PAF) fields. In all the examples, the SSA levels (CSI) increased during ACh injection, mainly due to an increase in the response to the deviant tone (red), rather than changes in the response to standard tones (blue).

\section{ACh increases the magnitude of the neuronal response and SSA}

To analyze the effect of ACh on SSA, we recorded the neuronal responses under an auditory oddball paradigm and computed the CSI to quantify the level of SSA. Fig. 3 illustrates five examples of units from each auditory cortical field recorded (PAF, A1, VAF, AAF and SRAF) before, during and after the injection of ACh. An increase in SSA levels is observed in all individual examples, mainly due to an increase in firing rate discharge to the deviant tone. ACh produced a significant increase of the CSI value in $83.8 \%$ of the neurons recorded (Fig. 4A, red dots; 83/99, bootstrapping, 95\% c.i.). Sixteen units (16.2\%) were unaffected by ACh and none showed a significant decrease of their CSI. 


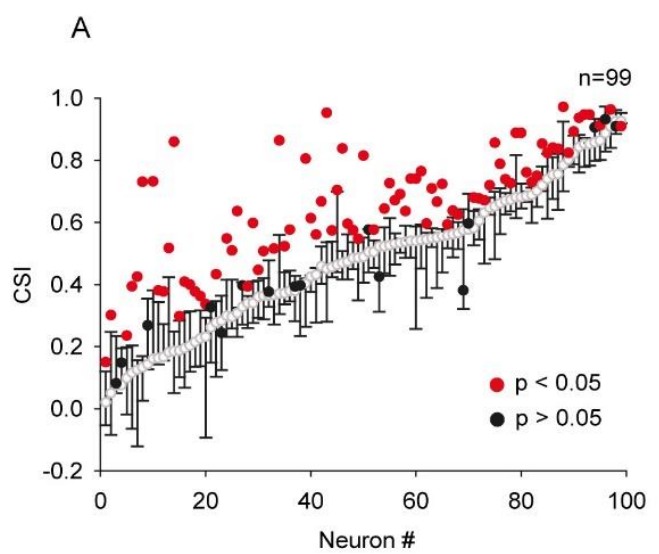

C
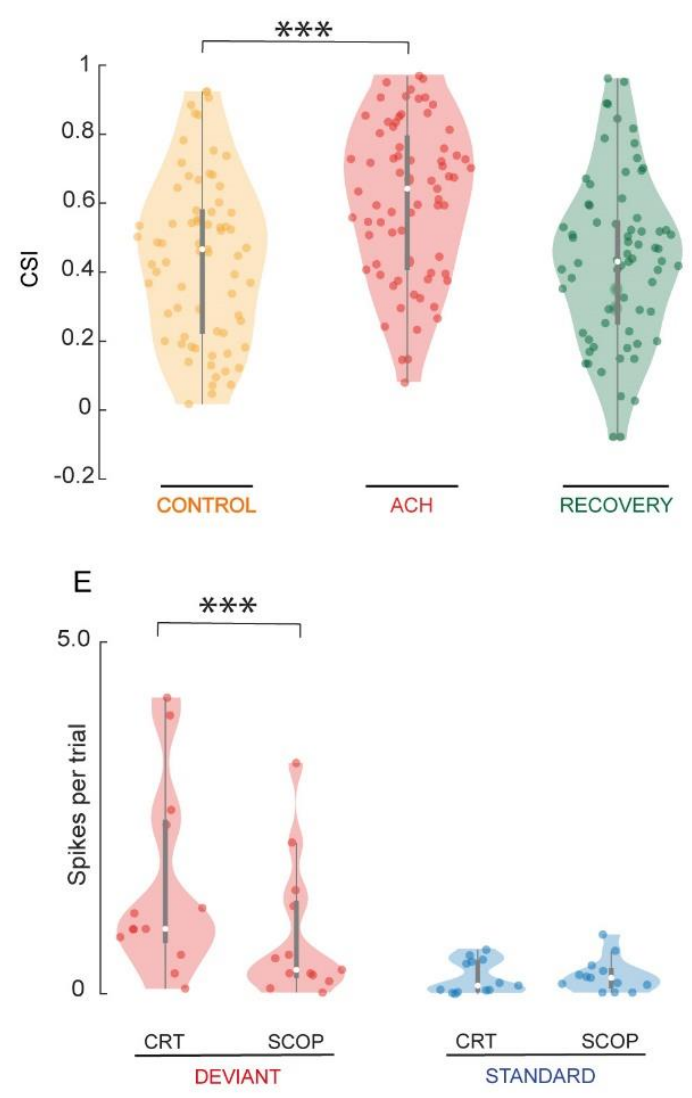

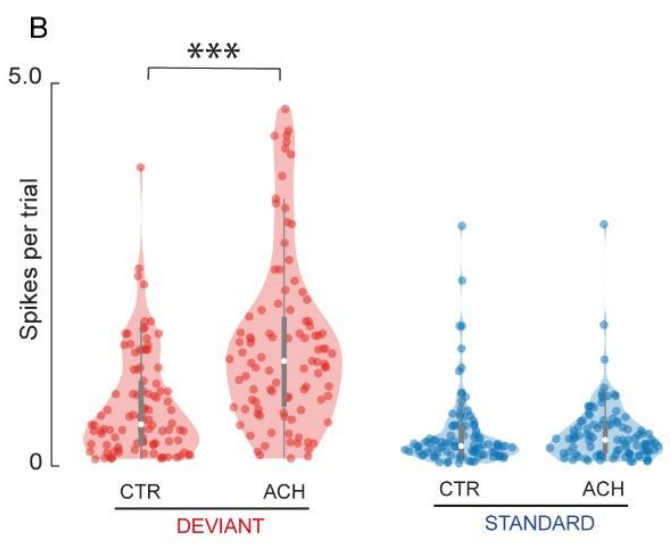

D
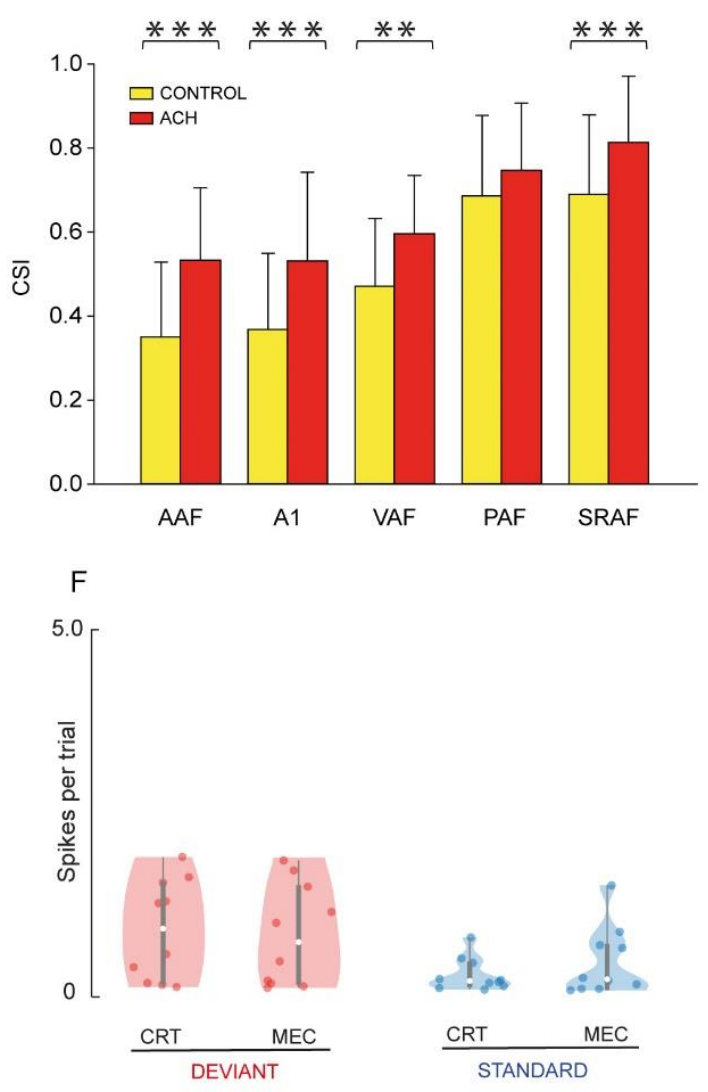

Figure 4: Effect of acetylcholine and cholinergic antagonists on SSA. (A)

Changes on the CSI due to the effect of ACh, for each recorded unit. The units are sorted based on their CSI in the control condition (white dots). In most of the units there was a significant change in CSI after ACh injection (83/99, red dots), while 16 units (black dots) did not change significantly. The vertical bars indicate the 95\% confidence interval for the CSI in the control condition. (B) Violin plots 
showing the distribution of responses to the deviant (red) and standard tones (blue) in control and ACh conditions. The application of ACh caused a significant increment in the response to deviant tones but not to standard tones. (C) Distribution of CSI values in control, ACh and recovery conditions. The application of ACh caused a significant increase in the CSI, which returned to basal levels afterwards. (D) The average CSI (mean + SD) increased significantly in all lemniscal cortical fields(AAF, Al, VAF) and in SRAF during ACh injection. (E) The application of the muscarinic antagonist scopolamine (SCOP) caused a significant decrement in the responses to deviant notes, but did not affect the responses to standard tones. $(F)$ In contrast, the application of the nicotinic antagonist mecamylamine (MEC) did not cause changes in the responses to neither deviant nor standard tones. In this and similar figures: ${ }^{*} p<0.05$; $* * p<0.01 ; * * * p<0.001$.

We analyzed the effect of ACh on the response to the deviant or standard stimuli separately. We observed that ACh produced a significant increase in the spike count in response to the deviant tones (Fig. 4B; $1.62 \pm 1.12$ spikes/stimulus), as compared to the control condition $(0.80 \pm 0.69$ spikes/stimulus; Wilcoxon Signed Rank test, $\mathrm{p}<0.001)$ while the magnitude of the response to standard tones remained unchanged (Fig. 3B; $0.40 \pm 0.49$ vs $0.41 \pm 0.42$ spikes/stimulus, control vs ACh; Wilcoxon Signed Rank test, $\mathrm{p}=0.08)$. These results clearly show that ACh has a differential effect in AC neurons, increasing the responses to rare and unexpected stimuli but not to common and expected stimuli.

Our sample $(n=99)$ spans a wide range of CSI values, from 0.02 to 0.93 , greatly enhancing our power to study the action of ACh. A remarkable finding was that ACh produced larger CSI increases to neurons with low SSAs ranging from 0.02 to 0.6 (CSI control: $0.37 \pm 0.16$ vs 
drug: $0.54 \pm 0.19 ; 71$ of 99 units). By contrast, neurons with CSI values larger than 0.6 showed smaller increases in SSA after ACh (CSI control: $0.76 \pm 0.10$ vs drug: $0.84 \pm 0.09 ; 28$ of 99 units). This may reflect a ceiling effect, as neurons with low levels of SSA have a larger range for SSA to increase. We found that the difference between control and ACh CSI levels for all units followed a linear distribution $\left(\mathrm{R}^{2}=0.17\right)$ and further support that $\mathrm{ACh}$ injection exerted larger changes on neurons with SSA levels below 0.6 (Fig 4A). This is in contrast with the effect of ACh in the inferior colliculus, where it has been shown to cause a larger increase of the CSI in neurons with SSA levels in the middle range (CSI $\approx 0.4-0.6)$ (Ayala and Malmierca, 2015).

The average CSI during ACh injection $(\mathrm{CSI}=0.62 \pm 0.22)$ was significantly larger than that of the control condition (CSI $=0.48 \pm 0.23$; Wilcoxon Signed Rank test, $\mathrm{p}<0.001$ ). Most of these neurons recovered their baseline CSI levels when the drug effects ended (CSI $=0.44 \pm$ 0.24; Fig. 4C). The effect of ACh on CSI was consistently observed on each individual AC field (Fig. 4D); although CSI values with ACh injection were lower in lemniscal (A1: 36 units, AAF: 20 units and VAF: 12 units) compared to non-lemniscal fields (PAF: 6 units and SRAF: 25 units) during the control condition.

\section{The effect of ACh on SSA in AC is mediated by muscarinic receptors}

Two major types of cholinergic receptors are present in the rat AC: muscarinic (mAChR) and nicotinic (nAChR). The nAChR are distributed mainly in layers I and II while mAChR are more widely distributed across layers (Colangelo et al., 2019; Edeline, 2012). To examine whether the effects of ACh were mediated by muscarinic or nicotinic receptors, we recorded from 23 additional neurons before, during and after the microiontophoretic injection of their corresponding antagonists: scopolamine and mecamylamine (Fig. 4E and 4F). Scopolamine significantly reduced the firing rate in response to deviant tones $(1.56 \pm 1.33$ vs $0.84 \pm 0.97$ spikes per trial; control vs scopolamine; Wilcoxon Signed Rank test, p < 0.001; Fig. 4E) 
without affecting the firing rate in response to standard tones $(0.26 \pm 0.24$ vs $0.29 \pm 0.24$ spikes per trial; control vs scopolamine; Wilcoxon Signed Rank test, $\mathrm{p}=0.216$ ). Accordingly, scopolamine reduced SSA levels in 11 of 13 neurons recorded (CSI: $0.63 \pm 0.14$ vs $0.55 \pm$ 0.15; control vs scopolamine; Wilcoxon Signed Rank test, $\mathrm{p}<0.001)$. Thus, the effect of scopolamine on firing rates and CSI values was opposite to the effect of acetylcholine. By contrast, mecamylamine (Fig. 4F) did not cause significant changes in the firing rates in response to neither the deviant $(0.91 \pm 0.69$ vs $0.87 \pm 0.70$; control vs mecamylamine; Wilcoxon Signed Rank test, $\mathrm{p}=0.91)$ nor the standard tones $(0.30 \pm 0.23$ vs $0.47 \pm 0.47$ spikes per trial; control vs mecamylamine; Wilcoxon Signed Rank test, $\mathrm{p}=0.28$ ). Therefore, mecamylamine did not affect SSA levels (CSI: $0.58 \pm 26$ vs $0.55 \pm 0.27$; control vs mecamylamine; Wilcoxon Signed Rank test, $\mathrm{p}=0.43$ ), suggesting the differential effect of ACh on the response to deviant stimuli is may be mediated by muscarinic receptors.

\section{ACh effect on SSA as a function of topographic distribution}

A remaining question is whether the effect of ACh is uniformly distributed across $\mathrm{AC}$ neurons in different fields and layers. Figure 5 shows the CSI and spike count levels for the deviant and standard tones before and after ACh application as well as the difference between the ACh and control conditions. The highest levels of CSI in the control condition (left column) were found in SRAF and PAF. After ACh application (middle column), the increase in CSI and the deviant spike counts were larger in the primary auditory fields (A1, AAF and VAF). By contrast, the ACh injection did not affect the spike count for the standard tone (middle column, bottom panel). When the responses during the control condition were subtracted from the ACh condition (right column), it became evident that the largest differences in the CSI were due to spike count increments in response to the deviant tone, as the activity for the 
standard tone remained largely unchanged.
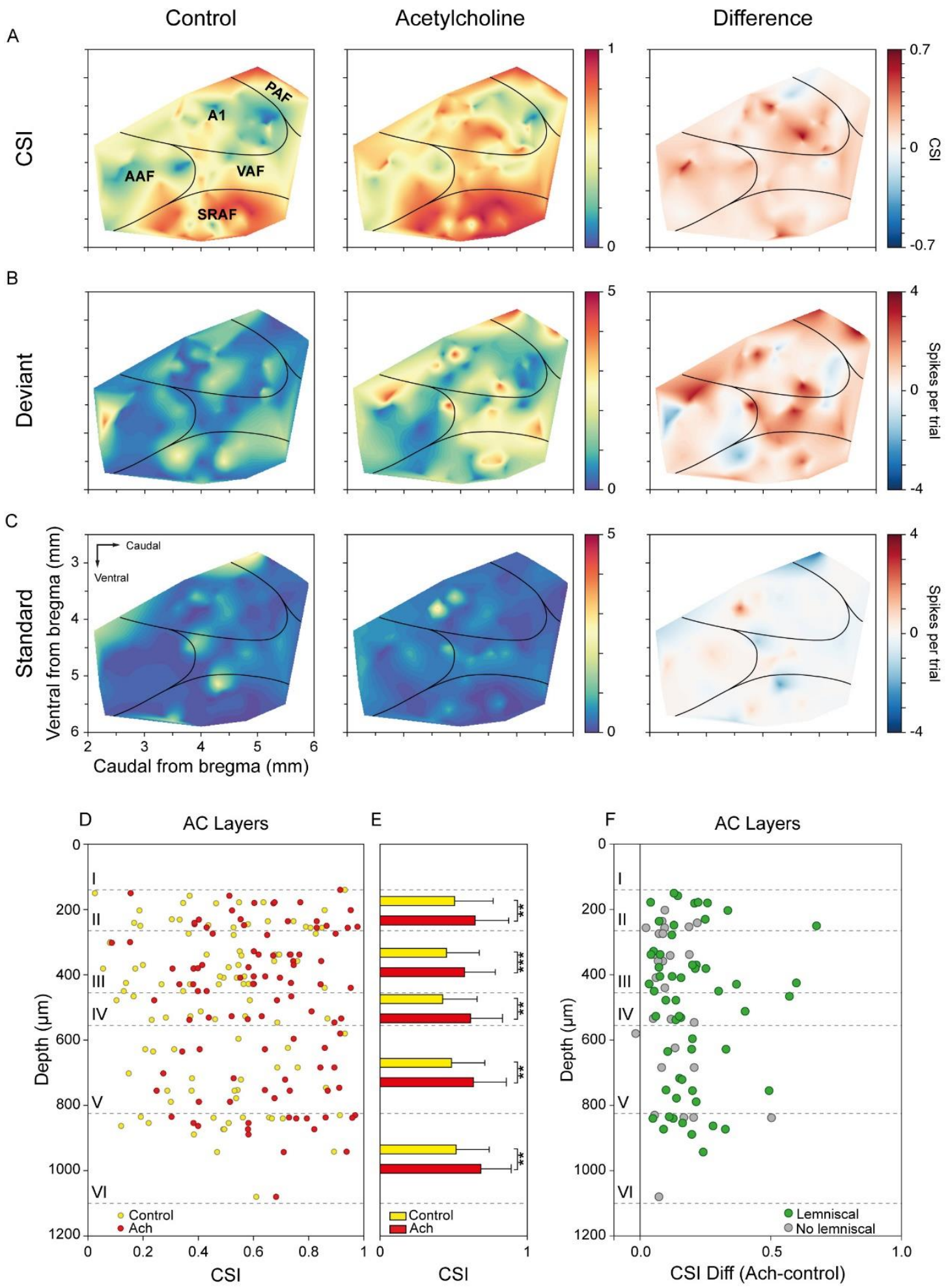

Figure 5: Anatomical localization of CSI and responses to the deviant and standard tones. Distribution of CSI (A) and responses (spikes per trial) for 
deviant $(B)$ and standard tones $(C)$, before and after ACh application, as well as the difference between both conditions. The highest levels of CSI are found in non-lemniscal fields, (SRAF and PAF). The application of ACh increased the response to the deviant tones throughout the AC but did not affect the responses to standard tones. (D) CSI levels of the recorded units according to their recording depth. The dashed lines indicate the approximate limits of the cortical layers. Yellow dots indicate the CSI in the control condition, while red dots indicate the corresponding CSI under the influence of ACh. We did not have any recordings that could be unambiguously located in layer $I .(E)$ Average (mean + SD) CSI in control (yellow) and ACh condition (red), for each cortical layer. $(F)$ Distribution of the difference between the levels of CSI obtained in the control and the ACh conditions (ACh-Control) for lemniscal (green) and non-lemniscal units (grey). Units with non-significant changes are excluded.

Figure 5D shows the distribution of CSI values as a function of recording depth within the AC under control conditions (yellow) and after the ACh injection (red). While CSI values were not different across layers in the control condition, they increased in all recorded layers (II to $\mathrm{VI}$ ) in response to ACh (layer II: $0.51 \pm 0.26$ vs $0.65 \pm 0.23$, control vs $\mathrm{ACh}, \mathrm{p}=0.001$; layer III: $0.45 \pm 0.22$ vs $0.57 \pm 0.21 ; p<0.001$; layer IV: $0.43 \pm 0.23$ vs $0.62 \pm 0.22, p<0.002$; layer $\mathrm{V}: 0.49 \pm 0.23$ vs $0.63 \pm 0.22, \mathrm{p}<0.001$; layer VI: $0.52 \pm 0.23$ vs $0.69 \pm 0.21 ; \mathrm{p}<0.001)$. The effect of ACh on the CSI across cortical layers was similar for lemniscal (Fig. 5F, green dots) and non-lemniscal (Fig. 5F, grey dots) fields (Mann-Whitney test, $\mathrm{p}=0.06$ ). Similarly, we found no differences in the CSI between supra- and infragranular layers of the AC (Mann- 
Whitney test, $\mathrm{p}=0.17$ ). In summary, these data demonstrate that ACh has a global and distributed effect on SSA regardless of the field or layer within the AC.

\section{ACh affects neuronal mismatch, increasing prediction error but not repetition}

\section{suppression}

Next, to test if ACh modulates PE signaling in AC, we recorded 65 additional neurons using the cascade control sequence as previously published (G. V Carbajal and Malmierca, 2018; Parras et al., 2017b; Ruhnau et al., 2012; Valdés-Baizabal et al., 2019) . Figures 6A and 6B show the spike counts in response to the control (CAS), deviant (DEV), and standard (STD) stimuli. ACh led to significant differences only in the DEV responses in the lemniscal fields (Wilcoxon Signed Rank test, $\mathrm{p}=0.013$ ). While there was a trend, the effect was not significant in the non-lemniscal fields (Wilcoxon Signed Rank test, $\mathrm{p}=0.066$ ), probably because the DEV responses were already strong during the control condition. 

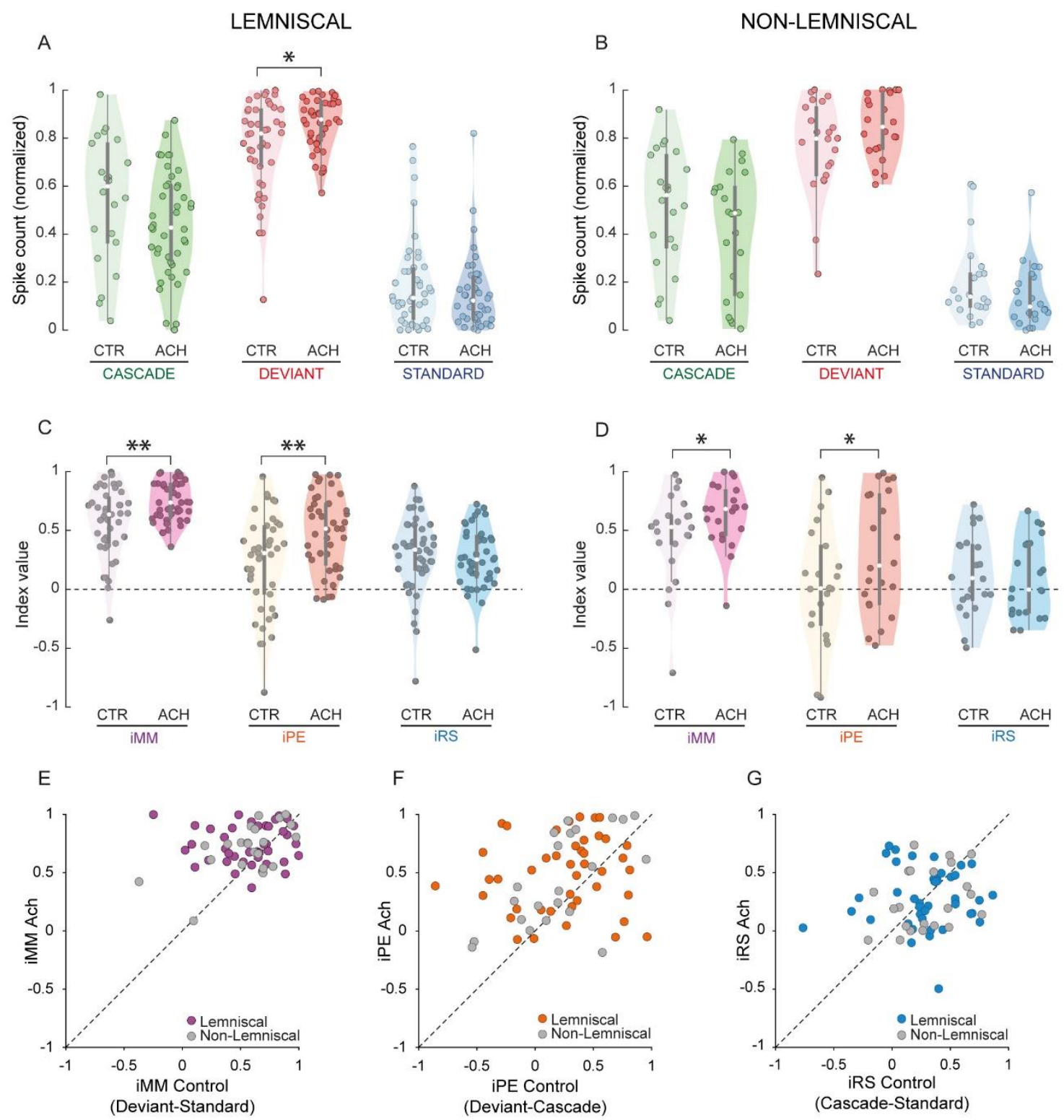

$\mathrm{H}$
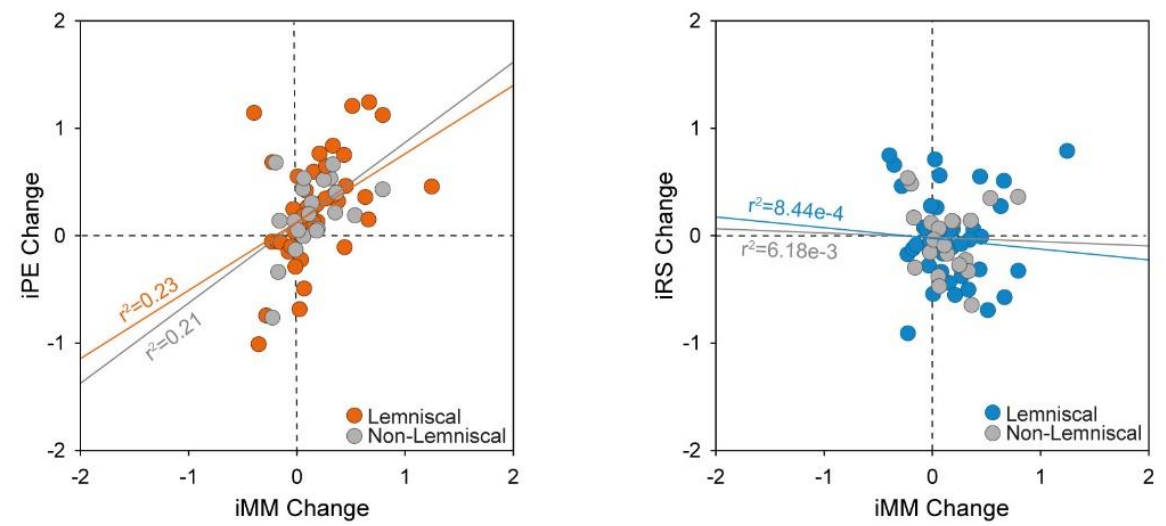

Figure 6: Predictive coding indices in lemniscal and non-lemniscal areas.

The application of ACh increased the normalized spike counts in response to 
deviant stimuli (red) in lemniscal (A) but not in non-lemniscal areas (B), and had no effect on the responses to neither the standard tones (blue) nor the cascade controls (green). In consequence, the neuronal mismatch (iMM, purple) and prediction error indexes (iPE, orange) increased significantly in both lemniscal $(C)$ and non-lemniscal areas $(D)$, but not the repetition suppression index (iRS, cyan). ( $E, F$ and $G)$ Scatter plots showing the effect of ACh for each individual unit on iMM (E), iPE $(F)$ and $i R S(G)$, from lemniscal (coloured dots) or non-lemniscal areas (gray dots). The changes of iPE due to the application of ACh $(H)$ were more correlated with the changes of iMM than the changes of iRS (I).

Using the normalized CAS, DEV and STD responses (see Methods), we computed the indexes iMM, iPE and iRS before and after the ACh injection in lemniscal (Figure 6C, iMM: $0.58 \pm 0.29$ vs $0.75 \pm 0.17$; iPE: $0.25 \pm 0.41$ vs $0.49 \pm 0.32 ;$ iRS: $0.31 \pm 0.32$ vs $0.28 \pm 0.25$; control vs ACh, respectively) and non-lemniscal fields (Figure 6D, iMM: $0.56 \pm 00.29$ vs 0.75 \pm 0.17 ; iPE: $0.22 \pm 0.40$ vs $0.44 \pm 0.40$; iRS: $0.31 \pm 0.27$ vs $0.27 \pm 0.26)$. Significant effects of ACh administration were found for iMM and iPE, both in lemniscal (iPE: $p=0.006$; iMM: $p$ $=0.005$; Wilcoxon Signed Rank test; Fig 6C) and non-lemniscal areas (iPE: $\mathrm{p}=0.015$; iMM: $\mathrm{p}=$ 0.026; Wilcoxon Signed Rank test; Fig 6D), while iRS remained unchanged (lemniscal iRS: $p=0.41$; non-lemniscal iRS: $p=0.53$; Wilcoxon Signed Rank test). Figure 6E-G illustrate the changes that occurred between the control and ACh conditions for the iMM, iPE and iRS respectively, both for the lemniscal (grey dots) and non-lemniscal fields (coloured dots). iMM and iPE values tend to lie above the main diagonal while the iRS values are distributed throughout the plot, i.e., ACh increased the magnitude of the $\mathrm{iMM}$ and this scaling is mostly due to an increase in the iPE. To further demonstrate that the scaling effect of ACh 
is on $\mathrm{iPE}$ and not on $\mathrm{iRS}$ we performed a regression analysis to examine the correlation between changes on the iMM and changes in either the iPE or the iRS. The linear model demonstrated a direct relationship between iMM and iPE changes (Fig 6H) while no apparent relationship existed between iMM and iRS changes (Fig 6I).

The models for the lemniscal fields (Fig. 6H-I, colored dots and lines) were:

$$
\begin{gathered}
i P E_{\text {change }}=0.12+0.75\left(i M_{\text {change }}\right) ; R^{2}=0.23 \\
i R S_{\text {change }}=-0.02-0.04\left(i M M_{\text {change }}\right) ; R^{2}=8.44 e-4
\end{gathered}
$$

While the models for the non-lemniscal fields (Fig. 6I, grey dots and lines) were:

$$
\begin{gathered}
i P E_{\text {change }}=0.13+0.64\left(i M M_{\text {change }}\right) ; R^{2}=0.21 \\
i R S_{\text {change }}=-0.02-0.10\left(i M M_{\text {change }}\right) ; R^{2}=6.18 e-3
\end{gathered}
$$

\section{DISCUSSION}

We recorded multiunit activity from AC neurons under an auditory oddball paradigm while performing microiontophoretic applications of ACh and corresponding muscarinic and nicotinic receptor antagonists to investigate the role of the cholinergic system in the processing of unexpected or surprising events. Furthermore, we employed cascade sequences to control for repetition suppression and distinctly assess cholinergic effects on prediction error relative to repetition suppression. Our results show that 1) ACh augments SSA by specifically increasing the response to the deviant tone (Fig. 7); 2) The opposite of this effect is found with the administration of a muscarinic antagonist, suggesting that this effect may be mediated by muscarinic ACh receptors; 3) ACh exerts a global and distributed effect on most AC neurons regardless of their field and layer location. Together, these findings reveal how ACh scales prediction error signals in the 
AC, likely gating these signals beyond the auditory cortex to frontal, higher cognitive regions.

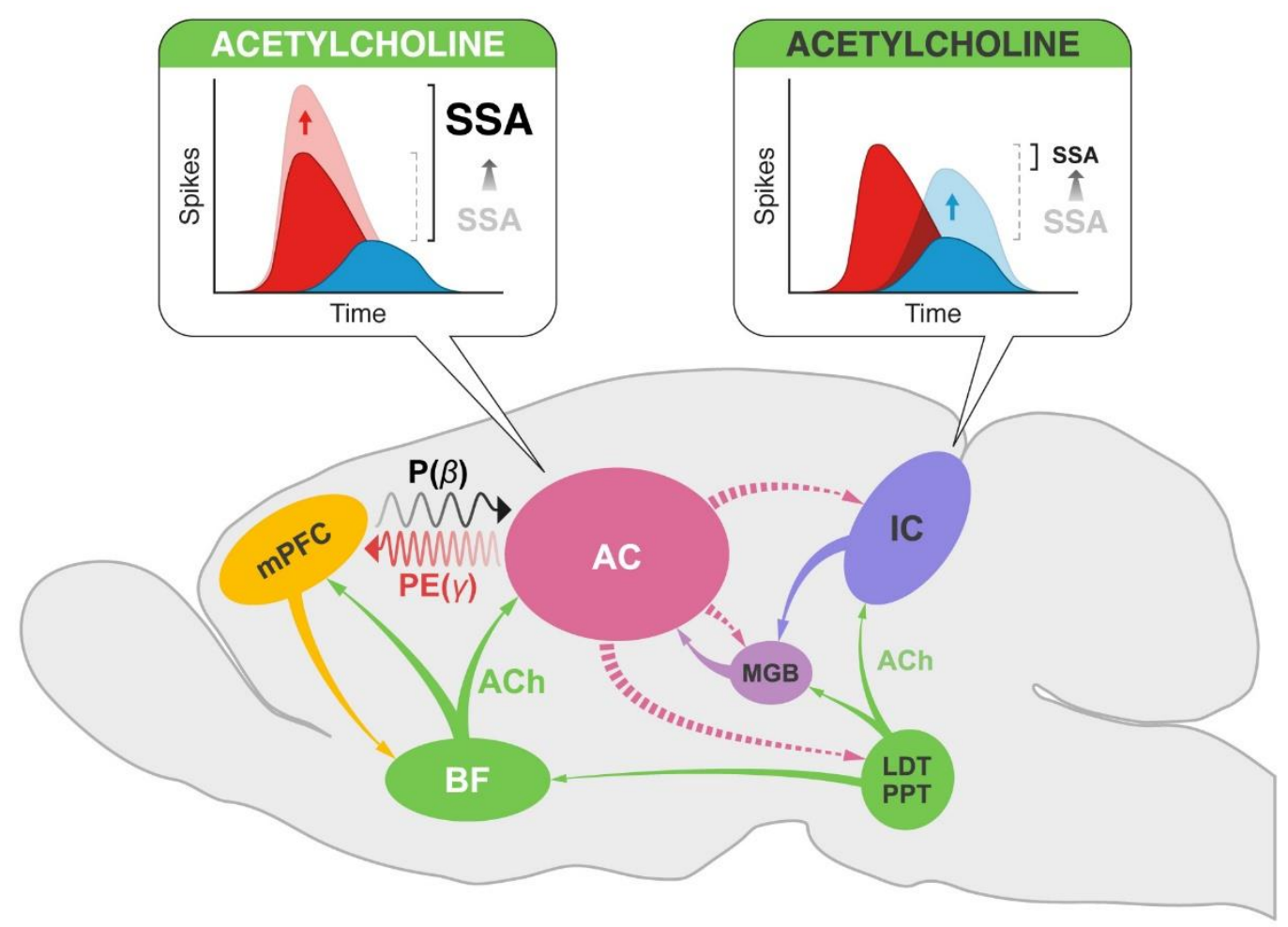

Figure 7: Effect of ACh on SSA in auditory midbrain and cortex. ACh increases SSA in AC scaling the firing rate in response to the 'deviants', while it shows an opposite effect in the IC, as ACh decreases SSA by scaling the responses to the 'standard' tones (Ayala and Malmierca, 2015). These divergent effects may be explained by the different connectivity of both auditory areas. While the $A C$ and medial prefrontal cortex ( $m P F C)$ receive their cholinergic input from the basal forebrain $(B F)$, the IC is innervated by the pedunculopontine (PPT) and laterodorsal tegmental (LDT) regions in the brainstem. This intersection of auditory and cholinergic nuclei creates an intricate network involving ascending and descending projections that 
ultimately modulate the processing of auditory deviance detection, such that

ACh increases the precision of prediction error signaling in AC, gating prediction errors (PE) to hierarchically higher processing levels ( $m P F C)$.

The present results are consistent with previous studies on the general effects of ACh on AC neurons, that found a facilitatory effect of ACh in most AC neurons. ACh has been shown to increase the amplitude of AC neuronal responses to sound stimulation, decreases the auditory threshold and sharpens the receptive field in the AC. (Edeline, 2012; Irvine, 2018b, 2018a; Kilgard and Merzenich, 1998; Ma and Suga, 2005; Metherate, 2011; Metherate et al., 1992; Puckett et al., 2007). These ACh-mediated effects are produced by a rapid disinhibition of neuronal responses, modifying synaptic strength, enhancing excitatory-inhibitory balance and reorganizing cortical circuits promoting cortical plasticity (Froemke et al., 2007; Irvine, 2018b, 2018a; Metherate, 2011; Picciotto et al., 2012). ACh plays important roles in arousal, attention, and sensory learning (Hasselmo, 1999; Hasselmo and Sarter, 2011; Metherate, 2011; Picciotto et al., 2012; Sarter et al., 2001; Weinberger, 2004). Thus, it is not surprising that ACh may have a critical role in shaping SSA, which has many properties in common with behavioral habituation to a repeated stimulus and can be considered a simple form of learning. (Irvine, 2018b; Nelken, 2014; Netser et al., 2011). But this is the first study, to our knowledge, that demonstrates a specific effect of ACh on prediction error responses.

ACh can exert its effects via mAChRs or nAChRs. We found that blocking muscarinic receptors with scopolamine led to a significant decrease in the neuronal firing rate in response to the deviant tone. In contrast, the nAChR blocker, mecamylamine, had no effect. Interestingly, the fact that blocking $\mathrm{mAChRs}$ only reduced the responses to deviant tones (without the application of $\mathrm{ACh}$ ) indicates that there is intrinsic release of ACh suggesting that such a release may be specifically linked to the occurrence of the deviant tones (Fig. 7). 
These results are similar to those from a similar study in the auditory midbrain (Ayala and Malmierca, 2015), where scopolamine, but not mecamylamine, affected SSA in the inferior colliculus (IC). However, in the IC, ACh selectively increased the response to the repetitious standard stimulus (Ayala and Malmierca, 2015), while we show here that ACh in the AC only increases the responses to the unexpected deviant (Fig. 7). This divergent effect of ACh on subcortical and cortical SSA may be related to the different origin of ACh and/or the unique organization of neuronal circuitries in IC and AC (Fig. 7). While the sources of ACh to IC emerge from the pedunculopontine and laterodorsal tegmental regions in the brainstem (Motts and Schofield, 2009), those to AC originate in the basal forebrain (Chavez and Zaborszky, 2017). The differential distribution of mAChRs and nAChRs in AC and IC is also noteworthy. While nAChRs are most abundant in supragranular layers of the cortex, mAChRs can be found throughout all cortical layer (Colangelo et al., 2019; Edeline, 2012). This is consistent with our finding that neurons whose SSA was affected by ACh were found similarly in all cortical layers, supporting the idea that ACh effect on SSA is mostly mediated by mAChRs and not by nAChRs. Moreover, this mAChRs influence on SSA would increase the cortical coding capacity of the deviant stimulus through an enhancement of postsynaptic excitability (Goard and Dan, 2009; Metherate, 2011).

Overall, the main effect of ACh was an increase in neuronal firing. Strong cholinergic activation, originating in the basal forebrain, is associated with active arousal states (BatistaBrito et al., 2018; Hasselmo, 1999). Likewise, cortical inhibitory interneurons play a crucial role in sensory processing and regulate what is represented and transmitted throughout cortical circuits. In this context, it is interesting to mention that recent studies have revealed and characterized the details of organization of canonical cortical circuits that underlie the translation of neuromodulatory signals into changes in sensory processing (reviewed in.(Batista-Brito et al., 2018; Picciotto et al., 2012) ).Thus, ACh can directly affect the 
responses of pyramidal neurons in AC, boosting response gain (Batista-Brito et al., 2018; Noudoost and Moore, 2011; Wood et al., 2017). ACh can also indirectly disinhibit pyramidal neurons through the activation of vasoactive intestinal peptide (VIP) interneurons, which express cholinergic receptors (Tremblay et al., 2016); VIP interneurons strongly inhibit somatostatin (SST) interneurons, that in turn inhibit pyramidal neurons. Yet another possibility is that ACh can directly activate SST interneurons, which inhibit parvalbumin (PV) interneurons (Xu et al., 2013), producing a similar disinhibitory effect on pyramidal neurons. In addition, it has been shown that optogenetic photosuppression of PV-mediated inhibition in AC leads to a nonspecific increase of neuronal responses, enhancing equally the responses to deviant and standard tones, while similar optogenetic photosuppression of SST-mediated inhibition selectively reduces excitatory responses to frequent tones (Natan et al., 2015). Moreover, long-lasting habituation involves a selective increase in SST-mediated inhibition (Kato et al., 2015). Thus, there are multiple opportunities by which ACh can modulate SSA, acting through several mechanisms and microcircuits involving VIP, SST and PV inhibitory interneurons. Future studies on the effect on noradrenergic input from the locus coeruleus to AC should be also considered as they also play a role in active arousal states (Batista-Brito et al., 2018; Hasselmo, 1999). Regardless of which specific circuits are involved, here we show that cholinergic modulation produces an increase in sensory gain in $\mathrm{AC}$, by increasing the firing rate in response to the deviant tone ACh (Muñoz et al., 2017; Muñoz and Rudy, 2014). This, in turn, may increase the impact of top-down inputs, promoting favorable conditions for changes in cortical plasticity such as SSA (Batista-Brito et al., 2018).

According to the predictive coding theory, cortical areas send prediction information to lower hierarchical areas to suppress expected neural activity and thus help anticipate sensory events. Conversely, those lower areas send error information to higher hierarchical centers when event predictions fail. If a stimulus is unexpected, the difference between expected and 
received inputs yields large prediction errors, while if a stimulus is constantly repeated, it yields a decrease in neural responsivity a phenomenon referred to as repetition suppression (Auksztulewicz and Friston, 2016). Thus, SSA can be explained under the predictive coding framework (G. V Carbajal and Malmierca, 2018; Parras et al., 2017b). However, predictions and prediction errors may also be modulated in a context-dependent manner, meaning that responses can be modulated and given precedence depending on the context in which the stimulus is perceived (Keller and Mrsic-Flogel, 2018). The source of such a modulating or gating signal may arise from neuromodulatory inputs (Fu et al., 2014; Moran et al., 2013; Pinto et al., 2013; Polack et al., 2013; Thiele and Bellgrove, 2018) that can not only gate plasticity (Kilgard and Merzenich, 1998; Martins and Froemke, 2015; Weinberger, 2004), but also change the balance of top-down versus bottom-up influence (Yu and Dayan, 2002). Within the predictive coding framework, classical neuromodulators such as ACh are often thought to increase the precision of prediction error signaling (Auksztulewicz et al., 2018; Auksztulewicz and Friston, 2016; Feldman and Friston, 2010; Moran et al., 2013), and this is precisely what our results confirm empirically at the neuronal level.

Using computational neuronal modeling and EEG recordings under oddball paradigms in subjects manipulated pharmacologically with galantamine (a competitive inhibitor of acetylcholinesterase, Moran et al., 2013) have shown that ACh enhances the precision of bottom-up synaptic transmission in cortical hierarchies by optimizing the gain of supragranular pyramidal neurons, amounting to an increase in the precision of prediction error signalling. Thus, our results and those from Moran and colleagues (2013)(Moran et al., 2013) suggest that $\mathrm{ACh}$ mediates the representation of precision and acts to facilitate the bottom-up signaling of sensory information (through precise prediction errors). In this context, Yu and Dayan (Yu and Dayan, 2005, 2002) proposed that ACh levels reflect the "expected 
uncertainty'" (when there is less confidence in the prediction) associated with top-down information and modulate the interaction between top-down and bottom-up processing in determining the appropriate neural representations for inputs (Auksztulewicz et al., 2017; Yu and Dayan, 2005, 2002)

Recent studies have emphasized the relationship between global networks in neural oscillations and predictive coding during stimulus processing (Chao et al., 2018; Sikkens et al., 2019). Indeed, a large body of evidence, including studies in both primates and humans, have proven that feedforward, and feedback communication between cortical areas is transmitted by gamma and alpha/beta-band oscillations, respectively (Bastos et al., 2012b; Dürschmid et al., 2016; Fontolan et al., 2014; Michalareas et al., 2016; Recasens et al., 2018; Sedley et al., 2016). Gamma oscillations have been found recently to be dependent on SST neuronal activity (Veit et al., 2017) and several human studies using ECoG and MEG have demonstrated the correlation between prediction errors and the magnitude of gamma-band oscillations in audition (Dürschmid et al., 2016; Edwards et al., 2005; Todorovic et al., 2011), and in other sensory systems (Auksztulewicz et al., 2017). ACh activation maintains a high postsynaptic gain and facilitates a spectral shift of neural oscillations toward higher (gammaband) frequencies (Liljenstrom and Hasselmo, 1995; Moran et al., 2013). Our finding that ACh gates neuronal prediction errors in the AC further supports the notion that activity in the gamma frequency band is linked to error signals originating from sensory areas like AC (Fig. 7), which convey prediction errors towards hierarchically higher brain areas.

In conclusion, we have shown that ACh plays a unique role in augmenting SSA in AC, increasing the precision of prediction error signaling and gating prediction errors to hierarchically higher processing levels, while possibly participating in the generation of gamma oscillations. 


\section{MATERIALS AND METHODS}

\section{Subjects and surgical procedures}

The experimental protocols were approved conforming to the University of Salamanca Animal Care Committee standards and the European Union (Directive 2010/63/EU) for the use of animals in neuroscience research. Experiments were performed on 30 adult female Long-Evans rats with body weights within 180-250 g. Surgical anesthesia was induced and maintained with urethane $(1.5 \mathrm{~g} / \mathrm{kg}$, intraperitoneal $)$, with supplementary doses $(0.5 \mathrm{~g} / \mathrm{kg}$, intraperitoneal $)$ given as needed. Dexamethasone $(0.25 \mathrm{mg} / \mathrm{kg})$ and atropine sulfate $(0.1$ $\mathrm{mg} / \mathrm{kg}$ ) were administered at the beginning of the surgery to reduce brain edema and the viscosity of bronchial secretions, respectively. Normal hearing was verified with auditory brainstem responses (ABR) recorded with subcutaneous needle electrodes, using a RZ6 Multi I/O Processor (Tucker-Davis Technologies, TDT) and processed with BioSig software (TDT). ABR stimuli consisted of $100 \mu$ s clicks at a 21/s rate, delivered monaurally to the right ear in $10 \mathrm{~dB}$ steps, from 10 to 90 decibels of sound pressure level (dB SPL), using a closed-field speaker. After the animal reached a surgical plane of anesthesia, the trachea was cannulated for artificial ventilation and a cisternal drain was introduced to prevent brain hernia and bran edema. Isotonic glucosaline solution was administered periodically $(5-10 \mathrm{ml}$ every $7 \mathrm{~h}$, subcutaneous) throughout the experiment to prevent dehydration. Body temperature was monitored with a rectal probe thermometer and maintained between 37 and $38^{\circ} \mathrm{C}$ with a homoeothermic blanket system.

The auditory cortex surgery was described in previous works (Nieto-Diego and Malmierca, 2016; Parras et al., 2017b) . The temporal bone was exposed and the auditory cortex was located using stereotactic coordinates (Paxinos et al., 1997). A craniotomy was performed 
over the auditory cortex, the dura was removed carefully, and the exposed area was filled with a layer of agar to prevent desiccation and to stabilize the recordings. Before applying the agar, a magnified picture $(25 \times)$ of the exposed cortex was taken with a digital camera coupled to the surgical microscope (Zeiss) through a lens adapter (TTI Medical). The picture included a pair of reference points previously marked on the dorsal ridge of the temporal bone, indicating the absolute scale and position of the image with respect to bregma (the reference point). This picture was displayed on a computer screen and was overlapped with a micrometric grid, over which the placement of the multibarrel for every recording was marked. The micrometric grid allowed to generate coordinates in a two-dimensional axis, from the superimposed image of the auditory cortex of each animal. Once the coordinates of each of the recorded units of all the animals were obtained, we used the functions 'griddata' and 'contourf' of MATLAB to generate topographic maps (through a graduation of colors) of the characteristic frequency (CF), Common SSA Index (CSI) levels, and deviant and standard tone responses in all auditory cortical fields.

\section{Electrophysiological recording and microiontophoresis}

A tungsten electrode (1-3 M $\Omega$ ) was used to record multiunit neuronal activity. It was attached to a 5-barrel multibarrel borosilicate glass pipette that carried drug solution to be delivered in the vicinity of the recorded neuron. The multibarrel's tip was cut to a diameter of about 20-30 $\mu \mathrm{m}$ approximately. The center barrel was filled with saline solution for current compensation $(165 \mathrm{mM} \mathrm{NaCl})$ whereas the others barrels were filled with $1 \mathrm{M}$ acetylcholine chloride (Sigma, catalog no. A6625), 0.5 M scopolamine hydrobromide (Sigma, catalog no. S0929), or 0.5 M mecamylamine hydrochloride (Tocris Bioscience, catalog no. 2843). The $\mathrm{pH}$ of acetylcholine, scopolamine and mecamylamine was adjusted between 4.0-4.2 (Ayala and Malmierca, 2015). Scopolamine and mecamylamine are antagonists of muscarinic and nicotinic receptors, respectively, whereas $\mathrm{ACh}$ is an agonist for both types of receptors. All 
drugs were retained by applying a -15 nA current, and were ejected when required, typically using 30-40 nA currents (Neurophore BH-2 System, Harvard Apparatus). The duration of the drug ejection usually lasted 8-10 min and the recording protocols were extended until the effect of the drug had disappeared (60 to 90 minutes approx.). The multibarrel assembly was positioned over the pial surface of the auditory cortex, forming a $30^{\circ}$ angle with the horizontal plane towards the rostral direction, and advanced using a piezoelectric micromanipulator (Sensapex) until we observed a strong spiking activity synchronized with the train of search stimuli. Analog signals were digitalized with a RZ6 Multi I/O Processor, a RA16PA Medusa Preamplifier and a ZC16 headstage (TDT) at $12 \mathrm{kHz}$ sampling rate and amplified 251x. Neurophysiological signals for multiunit activity were band-pass filtered between 0.5 and 4.5 $\mathrm{kHz}$.

\section{Experimental Design and stimulation paradigms}

Sound stimuli were generated using the RZ6 Multi I/O Processor (TDT) and custom software programmed with the OpenEx Suite (TDT) and MATLAB. Sounds were presented monaurally through a speaker, in a close-field condition to the ear contralateral to the left auditory cortex. We calibrated the speaker to ensure a flat spectrum up to $\sim 75 \mathrm{~dB}$ SPL between 0.5 and $44 \mathrm{kHz}$; the second and third harmonics were at least $40 \mathrm{~dB}$ lower than the fundamental at the loudest output level for all the frequencies. The experimental stimuli were pure tones in the range $0.5-44 \mathrm{kHz}$, with a duration of $75 \mathrm{~ms}$, including $5 \mathrm{~ms}$ rise/fall ramps presented at a rate of 4 stimuli/s. Once a suitable neuron was found, the frequency response area (FRA) of the cell, i.e. the combination of frequencies and intensities capable of evoking a suprathreshold response, was obtained automatically using a randomized paradigm that presented tones between $0.5-44 \mathrm{kHz}$ in 25 logarithmic steps, with intensities spaced by $10 \mathrm{~dB}$ steps, from 0 to $70 \mathrm{~dB}$ SPL, at a repetition rate of 4/s. Based on this information, we selected a pair of frequencies evoking similar responses at 10-30 dB above threshold. We used pure 
tones at these frequencies as the stimuli in the oddball paradigm. Oddball sequences consisted of frequently repeating stimuli (standard tones) which were pseudo-randomly interleaved with rare events (deviant tones). Two oddball sequences with fixed parameters (400 trials each, 75 ms stimulus duration, 0.5 octaves frequency separation, $10 \%$ deviant probability, $250 \mathrm{~ms}$ onset to onset, and a minimum of three standard tones before a deviant) were presented for every pair of stimuli thus selected. In one of the sequences, the low frequency $(f 1)$ was the "standard" and the high frequency $(f 2)$ was the "deviant," and in the other sequence their roles were inverted. The order of presentation of these two sequences was randomized across sites. In some cases, one or more extra pairs of stimuli were selected, and the oddball sequences were repeated with the new stimuli.

According to the predictive coding framework, mismatch responses like those obtained during an oddball paradigm can be divided in two components: repetition suppression (RS), a reduction in the response caused by a repeated stimulus, and prediction error (PE), an increased response caused by the violation of a regularity (G. V Carbajal and Malmierca, 2018). In a subset of the experiments, we used control sequences to evaluate the separate contribution of RS and PE. Control sequences consisted of 10 tones evenly spaced by 0.5 octaves (same as in the oddball sequences), including the tones used in the oddball paradigm, and all stimuli at the same previously chosen sound level. Each control sequence lasted 400 trials, the duration of all stimuli was $75 \mathrm{~ms}$ and the presentation rate 4/s. We used two different control sequences, namely the many-standards and cascade sequences (Supplementary Figure 1). The many-standards control is the consecutive presentation of blocks of 10 tones randomly ordered within the block, each tone with a $10 \%$ probability of occurrence (Schröger and Wolff, 1996). In this sequence the tones are unpredictable, and it is not possible to establish a rule which could be used to predict the following tones. On the other hand, the cascade control consists of the regular presentation of the same 10 tones in 
ascending or descending frequency succession (Ruhnau et al., 2012). This sequence also avoids the effects of the repetition of a single stimulus, but in this case, it maintains a predictable context. Cascade sequences are considered a more rigorous control than manystandard sequences because like the oddball sequence, a regularity is established (G. V Carbajal and Malmierca, 2018) .

\section{Data analysis}

The degree of SSA was quantified by the CSI, reported previously (Malmierca et al., 2009; Ulanovsky et al., 2003). The CSI reflects the difference between the neural responses to the deviant and standard stimuli, normalized to the total of responses to both stimuli, and is defined as:

$$
C S I=\frac{d\left(f_{1}\right)+d\left(f_{2}\right)-s\left(f_{1}\right)-s\left(f_{2}\right)}{d\left(f_{1}\right)+d\left(f_{2}\right)+s\left(f_{1}\right)+s\left(f_{2}\right)}
$$

where $d\left(f_{i}\right)$ and $s\left(f_{i}\right)$ are responses to each frequency $f_{1}$ or $f_{2}$ when they were the deviant (d) or standard ( $s$ ) stimulus in the oddball paradigm, respectively.

For the subset of experiments where we recorded the many-standards and cascade controls, we compared the responses to the same physical stimulus when it took the role of a standard, a deviant (ascending or descending, depending on whether the preceding standard was of lower or higher frequency, respectively), or was part of a cascade sequence (matching the ascending or descending condition of the corresponding deviant). Alongside the oddball paradigm, we recorded responses of neurons to two cascade sequences (ascending and descending), which consisted of 10 tones selected within the FRA presented in a predictable succession of increasing or decreasing frequencies. We did not include the many-standards control in these analyses because these experiments are time consuming and the need of holding the recording neuron for long enough before, during and after the drug injection. 
However, we have previously demonstrated that the many-standards and cascade controls responses were comparable, and the latter is considered to be more rigorous. (Casado-Román et al., 2019; Parras et al., 2017b; Ruhnau et al., 2012; Valdés-Baizabal et al., 2019).

The responses of each neuron were normalized as

$$
\begin{gathered}
\text { Normalized Deviant }=\text { Deviant } / N \\
\text { Normalized Standard }=\text { Standard } / N \\
\text { Normalized Control }=\text { Control } / N
\end{gathered}
$$

where

$$
N=\sqrt{\text { Deviant }^{2}+\text { Standard }^{2}+\text { Control }^{2}}
$$

is the Euclidean norm of the vector defined by the deviant, standard and cascade responses, so that the normalized responses take values in the range $0-1$.

With these normalized baseline-corrected spike counts, we next computed the indices of neuronal mismatch (iMM), repetition suppression (iRS), and prediction error (iPE) as:

$$
\begin{gathered}
i M M=\text { Normalized Deviant }- \text { Normalized Standard } \\
i R S=\text { Normalized Control }- \text { Normalized Standard } \\
i P E=\text { Normalized Deviant }- \text { Normalized Control }
\end{gathered}
$$

Index values ranged between -1 and 1 and facilitated the quantitative decomposition of neuronal mismatch into RS and PE since

$$
i M M=i R S+i P E
$$

Which is largely comparable to the CSI calculated for the oddball paradigm (Parras et al., 2017b). 
In order to determine a significant effect of the drugs on the CSI (Fig. 4A), we calculated an empirical distribution of CSI values by performing 2000 bootstraps of the responses to each standard or deviant stimulus for each neuron in the control condition, from which we determined a 95\% confidence interval (vertical lines in Fig. 4A). An effect was considered significant at $\alpha=0.05$ if the CSI during the drug condition fell beyond the limits of the control CSI confidence interval (red dots in Fig. 4A).

Since parameters did not follow a normal distribution, non-parametric statistics were used: Mann-Whitney (for independent data) or Wilcoxon Signed Rank test (for paired data). Multiple comparisons were corrected using the Holm-Bonferroni approach. All data are reported as mean $\pm \mathrm{SD}$. All of the data analyses were performed with Sigma Plot v12.5 and MATLAB software, using the built-in functions, the Statistics and Machine Learning toolbox, or custom scripts and functions developed in our laboratory.

Acknowledgments: We thank Drs. Ryszard Auksztulewicz, Yaneri A. Ayala, Donald Caspary, Diego Contreras, Lauren Harms and Marina Picciotto for their constructive criticisms on a previous version. This work was supported by the Spanish MINECO (SAF2016-75803-P) to MSM.

Competing interests: No competing interests declared 


\section{REFERENCES}

Andermann, M.L., Ritt, J., Neimark, M.A., Moore, C.I., 2004. Neural correlates of vibrissa resonance: Band-pass and somatotopic representation of high-frequency stimuli. Neuron 42, 451-463. https://doi.org/10.1016/S0896-6273(04)00198-9

Anderson, L.A., Malmierca, M.S., 2013. The effect of auditory cortex deactivation on stimulus-specific adaptation in the inferior colliculus of the rat. Eur. J. Neurosci. https://doi.org/10.1111/ejn.12018

Antunes, F.M., Malmierca, M.S., 2011. Effect of auditory cortex deactivation on stimulus-specific adaptation in the medial geniculate body. J. Neurosci. https://doi.org/10.1523/JNEUROSCI.1915-11.2011

Antunes, F.M., Nelken, I., Covey, E., Malmierca, M.S., 2010. Stimulus-specific adaptation in the auditory thalamus of the anesthetized rat. PLoS One. https://doi.org/10.1371/journal.pone.0014071

Askew, C.E., Metherate, R., 2016. Synaptic interactions and inhibitory regulation in auditory cortex. Biol. Psychol. 116, 4-9.

https://doi.org/10.1016/j.biopsycho.2015.11.001

Atencio, C.A., Schreiner, C.E., 2008. Spectrotemporal processing differences between auditory cortical fast-spiking and regular-spiking neurons. J. Neurosci. 28, 38973910. https://doi.org/10.1523/JNEUROSCI.5366-07.2008

Auksztulewicz, R., Friston, K., 2016. Repetition suppression and its contextual determinants in predictive coding. Cortex. https://doi.org/10.1016/j.cortex.2015.11.024

Auksztulewicz, R., Friston, K.J., Nobre, A.C., 2017. Task relevance modulates the behavioural and neural effects of sensory predictions. PLoS Biol. 15, e2003143. https://doi.org/10.1371/journal.pbio.2003143

Auksztulewicz, R., Schwiedrzik, C.M., Thesen, T., Doyle, W., Devinsky, O., Nobre, A.C., Schroeder, C.E., Friston, K.J., Melloni, L., 2018. Not all predictions are equal: "what" and "when" predictions modulate activity in auditory cortex through different mechanisms. J. Neurosci. 38, 8680-8693. https://doi.org/10.1523/JNEUROSCI.0369-18.2018

Ayala, Y.A., Malmierca, M.S., 2015. Cholinergic modulation of stimulus-specific adaptation in the inferior colliculus. J. Neurosci. 35, 12261-12272. https://doi.org/10.1523/JNEUROSCI.0909-15.2015

Ayala, Y.A., Pérez-González, D., Duque, D., Nelken, I., Malmierca, M.S., 2012. Frequency discrimination and stimulus deviance in the inferior colliculus and cochlear nucleus. Front. Neural Circuits. https://doi.org/10.3389/fncir.2012.00119

Baeg, E.H., Kim, B.K., Jang, J., Kim, H.T., Mook-Jung, I., Jung, M.W., 2001. Fast Spiking and Regular Spiking Neural Correlates of Fear Conditioning in the Medial Prefrontal Cortex of the Rat. Cereb. Cortex 11, 441-451. https://doi.org/10.1093/cercor/11.5.441

Bakin, J.S., Weinberger, N.M., 1990. Classical conditioning induces CS-specific receptive field plasticity in the auditory cortex of the guinea pig. Brain Res. 536, 271-286. https://doi.org/10.1016/0006-8993(90)90035-A

Barthó, P., Hirase, H., Monconduit, L., Zugaro, M., Harris, K.D., Buzsáki, G., 2004. Characterization of neocortical principal cells and interneurons by network interactions and extracellular features. J. Neurophysiol. 92, 600-608. https://doi.org/10.1152/jn.01170.2003

Bastos, A.M., Usrey, W.M., Adams, R.A., Mangun, G.R., Fries, P., Friston, K.J., 2012a. Canonical Microcircuits for Predictive Coding. Neuron. 
https://doi.org/10.1016/j.neuron.2012.10.038

Bastos, A.M., Usrey, W.M., Adams, R.A., Mangun, G.R., Fries, P., Friston, K.J., 2012b. Canonical Microcircuits for Predictive Coding. Neuron. https://doi.org/10.1016/j.neuron.2012.10.038

Batista-Brito, R., Zagha, E., Ratliff, J.M., Vinck, M., 2018. Modulation of cortical circuits by top-down processing and arousal state in health and disease. Curr. Opin. Neurobiol. https://doi.org/10.1016/j.conb.2018.06.008

Bendixen, A., 2014. Predictability effects in auditory scene analysis: A review. Front. Neurosci. https://doi.org/10.3389/fnins.2014.00060

Bentley, P., Driver, J., Dolan, R.J., 2011. Cholinergic modulation of cognition: Insights from human pharmacological functional neuroimaging. Prog. Neurobiol. https://doi.org/10.1016/j.pneurobio.2011.06.002

Bruno, R.M., Simons, D.J., 2002. Feedforward mechanisms of excitatory and inhibitory cortical receptive fields. J. Neurosci. 22, 10966-10975.

Calabrese, A., Woolley, S.M.N., 2015. Coding principles of the canonical cortical microcircuit in the avian brain. Proc. Natl. Acad. Sci. 112, 3517-3522. https://doi.org/10.1073/pnas.1408545112

Caldenhove, S., Borghans, L.G.J.M., Blokland, A., Sambeth, A., 2017. Role of acetylcholine and serotonin in novelty processing using an oddball paradigm. Behav. Brain Res. https://doi.org/10.1016/j.bbr.2017.05.031

Calford, M.B., Aitkin, L.M., 1983. Ascending projections to the medial geniculate body of the cat: Evidence for multiple, parallel auditory pathways through thalamus. J. Neurosci. 3, 2365-2380. https://doi.org/10.1523/jneurosci.03-11-02365.1983

Carbajal, Guillermo Varela, Malmierca, M.S., 2018. The unique role of the nonlemniscal pathway on stimulus-specific adaptation (SSA) in the auditory system, in: Proceedings of the International Symposium on Auditory and Audiological Research.

Carbajal, Guillermo V., Malmierca, M.S., 2018. The Neuronal Basis of Predictive Coding Along the Auditory Pathway: From the Subcortical Roots to Cortical Deviance Detection. Trends Hear. https://doi.org/10.1177/2331216518784822

Carbajal, G. V, Malmierca, M.S., 2018. The Neuronal Basis of Predictive Coding Along the Auditory Pathway: From the Subcortical Roots to Cortical Deviance Detection Introduction: SSA and MMN, Two Faces of Deviance Detection. journals.sagepub.com 22. https://doi.org/10.1177/2331216518784822

Casado-Román, L., Pérez-González, D., Malmierca, M.S., 2019. Prediction errors explain mismatch signals of neurons in the medial prefrontal cortex. bioRxiv 778928. https://doi.org/10.1101/778928

Ceballo, S., Piwkowska, Z., Bourg, J., Daret, A., Bathellier, B., 2019. Targeted Cortical Manipulation of Auditory Perception. Neuron 104, 1168-1179.e5. https://doi.org/10.1016/j.neuron.2019.09.043

Chao, Z.C., Takaura, K., Wang, L., Fujii, N., Dehaene, S., 2018. Large-Scale Cortical Networks for Hierarchical Prediction and Prediction Error in the Primate Brain. Neuron 100, 1252-1266.e3. https://doi.org/10.1016/j.neuron.2018.10.004

Chavez, C., Zaborszky, L., 2017. Basal Forebrain Cholinergic-Auditory Cortical Network: Primary Versus Nonprimary Auditory Cortical Areas. Cereb. Cortex 27, 2335-2347. https://doi.org/10.1093/cercor/bhw091

Chen, I.-W., Helmchen, F., Lütcke, H., 2015. Specific Early and Late Oddball-Evoked Responses in Excitatory and Inhibitory Neurons of Mouse Auditory Cortex. Soc Neurosci. https://doi.org/10.1523/JNEUROSCI.2240-15.2015

Chen, I.W., Helmchen, F., Lütcke, H., 2015. Specific early and late oddball-evoked 
responses in excitatory and inhibitory neurons of mouse auditory cortex. J.

Neurosci. 35, 12560-12573. https://doi.org/10.1523/JNEUROSCI.2240-15.2015

Colangelo, C., Shichkova, P., Keller, D., Markram, H., Ramaswamy, S., 2019. Cellular, synaptic and network effects of acetylcholine in the neocortex. Front. Neural Circuits. https://doi.org/10.3389/fncir.2019.00024

Coomes, D.L., Schofield, B.R., 2004. Separate projections from the inferior colliculus to the cochlear nucleus and thalamus in guinea pigs. Hear. Res.

https://doi.org/10.1016/j.heares.2004.01.009

Dalley, J.W., Cardinal, R.N., Robbins, T.W., 2004. Prefrontal executive and cognitive functions in rodents: Neural and neurochemical substrates, in: Neuroscience and Biobehavioral Reviews. pp. 771-784. https://doi.org/10.1016/j.neubiorev.2004.09.006

Disney, A.A., Aoki, C., Hawken, M.J., 2007. Gain Modulation by Nicotine in Macaque V1. Neuron. https://doi.org/10.1016/j.neuron.2007.09.034

Duque, D., Malmierca, M.S., Caspary, D.M., 2014. Modulation of stimulus-specific adaptation by GABAA receptor activation or blockade in the medial geniculate body of the anaesthetized rat. J. Physiol. 592, 729-743. https://doi.org/10.1113/jphysiol.2013.261941

Duque, D., Pérez-González, D., Ayala, Y.A., Palmer, A.R., Malmierca, M.S., 2012. Topographic distribution, frequency, and intensity dependence of stimulus-specific adaptation in the inferior colliculus of the rat. J. Neurosci. https://doi.org/10.1523/JNEUROSCI.3190-12.2012

Dürschmid, S., Edwards, E., Reichert, C., Dewar, C., Hinrichs, H., Heinze, H.J., Kirsch, H.E., Dalal, S.S., Deouell, L.Y., Knight, R.T., 2016. Hierarchy of prediction errors for auditory events in human temporal and frontal cortex. Proc. Natl. Acad. Sci. U. S. A. 113, 6755-6760. https://doi.org/10.1073/pnas.1525030113

Dutta, A., Gutfreund, Y., 2014. Saliency mapping in the optic tectum and its relationship to habituation. Front. Integr. Neurosci. https://doi.org/10.3389/fnint.2014.00001

Edeline, J.M., 2012. Beyond traditional approaches to understand the functional role of neuromodulators in sensory cortices. Front. Behav. Neurosci. https://doi.org/10.3389/fnbeh.2012.00045

Edwards, E., Soltani, M., Deouell, L.Y., Berger, M.S., Knight, R.T., 2005. High gamma activity in response to deviant auditory stimuli recorded directly from human cortex. J. Neurophysiol. 94, 4269-4280. https://doi.org/10.1152/jn.00324.2005

Eggermann, E., Kremer, Y., Crochet, S., Petersen, C.C.H., 2014. Cholinergic Signals in Mouse Barrel Cortex during Active Whisker Sensing. Cell Rep. https://doi.org/10.1016/j.celrep.2014.11.005

Eytan, D., Brenner, N., Marom, S., 2003. Selective Adaptation in Networks of Cortical Neurons. J. Neurosci. 23, 9349-9356. https://doi.org/10.1523/jneurosci.23-2809349.2003

Farley, B.J., Quirk, M.C., Doherty, J.J., Christian, E.P., 2010. Stimulus-specific adaptation in auditory cortex is an NMDA-independent process distinct from the sensory novelty encoded by the mismatch negativity. J. Neurosci. 30, 1647516484. https://doi.org/10.1523/JNEUROSCI.2793-10.2010

Feldman, H., Friston, K.J., 2010. Attention, uncertainty, and free-energy. Front. Hum. Neurosci. 4. https://doi.org/10.3389/fnhum.2010.00215

Fontolan, L., Morillon, B., Liegeois-Chauvel, C., Giraud, A.L., 2014. The contribution of frequency-specific activity to hierarchical information processing in the human auditory cortex. Nat. Commun. 5, 1-10. https://doi.org/10.1038/ncomms5694 
Franklin, N.T., Frank, M.J., 2015. A cholinergic feedback circuit to regulate striatal population uncertainty and optimize reinforcement learning. Elife 4. https://doi.org/10.7554/eLife.12029

Friston, K., 2010. The free-energy principle: A unified brain theory? Nat. Rev. Neurosci. https://doi.org/10.1038/nrn2787

Friston, K., 2008. Hierarchical Models in the Brain. Cit. Frist. K 4, 1000211. https://doi.org/10.1371/journal.pcbi.1000211

Friston, K., 2005. A theory of cortical responses. royalsocietypublishing.org 360, 815836. https://doi.org/10.1098/rstb.2005.1622

Friston, K., Kiebel, S., 2009. Cortical circuits for perceptual inference. Neural Networks 22, 1093-1104. https://doi.org/10.1016/j.neunet.2009.07.023

Fritz, J., Elhilali, M., Shamma, S., 2005. Active listening: Task-dependent plasticity of spectrotemporal receptive fields in primary auditory cortex. Hear. Res. 206, 159176. https://doi.org/10.1016/j.heares.2005.01.015

Fritz, J., Shamma, S., Elhilali, M., Klein, D., 2003. Rapid task-related plasticity of spectrotemporal receptive fields in primary auditory cortex. Nat. Neurosci. 6, 1216-1223. https://doi.org/10.1038/nn1141

Froemke, R.C., Merzenich, M.M., Schreiner, C.E., 2007. A synaptic memory trace for cortical receptive field plasticity. Nature 450, 425-429. https://doi.org/10.1038/nature06289

Fu, Y., Tucciarone, J.M., Espinosa, J.S., Sheng, N., Darcy, D.P., Nicoll, R.A., Huang, Z.J., Stryker, M.P., 2014. A cortical circuit for gain control by behavioral state. Cell 156, 1139-1152. https://doi.org/10.1016/j.cell.2014.01.050

Games, K.D., Winer, J.A., 1988. Layer V in rat auditory cortex: Projections to the inferior colliculus and contralateral cortex. Hear. Res. https://doi.org/10.1016/0378-5955(88)90047-0

Garrido, M.I., Kilner, J.M., Kiebel, S.J., Friston, K.J., 2009a. Dynamic causal modeling of the response to frequency deviants. J. Neurophysiol. 101, 2620-2631. https://doi.org/10.1152/jn.90291.2008

Garrido, M.I., Kilner, J.M., Stephan, K.E., Friston, K.J., 2009b. The mismatch negativity: A review of underlying mechanisms. Clin. Neurophysiol. https://doi.org/10.1016/j.clinph.2008.11.029

Ghazanfar, A.A., Schroeder, C.E., 2006. Is neocortex essentially multisensory? Trends Cogn. Sci. https://doi.org/10.1016/j.tics.2006.04.008

Goard, M., Dan, Y., 2009. Basal forebrain activation enhances cortical coding of natural scenes. Nat. Neurosci. 12, 1444-1449. https://doi.org/10.1038/nn.2402

Gottlieb, Y., Vaadia, E., Abeles, M., 1989. Single unit activity in the auditory cortex of a monkey performing a short term memory task. Exp. Brain Res. 74, 139-148. https://doi.org/10.1007/BF00248287

Guillermo V. Carbajal and Manuel S. Malmierca, 2020. Novelty Processing in the Auditory System: Detection, Adaptation or Expectation?, in: Grothe Benedick, F.B. (Ed.), The Senses: A Comprehensive Reference, 2nd Edition. Elsevier.

Gutfreund, Y., 2012. Stimulus-specific adaptation, habituation and change detection in the gaze control system. Biol. Cybern. 106, 657-668. https://doi.org/10.1007/s00422-012-0497-3

Hackett, T.A., 2015. Anatomic organization of the auditory cortex, in: Handbook of Clinical Neurology. https://doi.org/10.1016/B978-0-444-62630-1.00002-0

Hara, K., Harris, R.A., 2002. The anesthetic mechanism of urethane: the effects on neurotransmitter-gated ion channels. Anesth Analg 94, 313-318.

Hardy, C.J.D., Yong, K.X.X., Goll, J.C., Crutch, S.J., Warren, J.D., 2020. Impairments 
of auditory scene analysis in posterior cortical atrophy. Brain 143, 2689-2695. https://doi.org/10.1093/brain/awaa221

Harms, L., Parras, G.G., Michie, P.T., Malmierca, M.S., 2020. The Role of Glutamate Neurotransmission in Mismatch Negativity (MMN), A Measure of Auditory Synaptic Plasticity and Change-detection. Neuroscience. https://doi.org/10.1016/j.neuroscience.2020.01.046

Hasselmo, M.E., 1999. Neuromodulation: Acetylcholine and memory consolidation. Trends Cogn. Sci. https://doi.org/10.1016/S1364-6613(99)01365-0

Hasselmo, M.E., Giocomo, L.M., 2006. Cholinergic modulation of cortical function, in: Journal of Molecular Neuroscience. pp. 133-135. https://doi.org/10.1385/JMN:30:1:133

Hasselmo, M.E., Sarter, M., 2011. Modes and models of forebrain cholinergic neuromodulation of cognition. Neuropsychopharmacology. https://doi.org/10.1038/npp.2010.104

Hefti, B.J., Smith, P.H., 2000. Anatomy, physiology, and synaptic responses of rat layer $\mathrm{V}$ auditory cortical cells and effects of intracellular GABA(A) blockade. J. Neurophysiol. 83, 2626-2638. https://doi.org/10.1152/jn.2000.83.5.2626

Hubel, D.H., Henson, C.O., Rupert, A., Galambos, R., 1959. "Attention" units in the auditory cortex. Science (80-. ). 129, 1279-1280. https://doi.org/10.1126/science.129.3358.1279

Insel, N., Barnes, C.A., 2015. Differential activation of fast-spiking and regular-firing neuron populations during movement and reward in the dorsal medial frontal cortex. Cereb. Cortex 25, 2631-2647. https://doi.org/10.1093/cercor/bhu062

Irvine, D.R.F., 2018a. Plasticity in the auditory system. Hear. Res. https://doi.org/10.1016/j.heares.2017.10.011

Irvine, D.R.F., 2018b. Auditory perceptual learning and changes in the conceptualization of auditory cortex. Hear. Res. https://doi.org/10.1016/j.heares.2018.03.011

Ishishita, Y., Kunii, N., Shimada, S., Ibayashi, K., Tada, M., Kirihara, K., Kawai, K., Uka, T., Kasai, K., Saito, N., 2019. Deviance detection is the dominant component of auditory contextual processing in the lateral superior temporal gyrus: A human ECoG study. Hum. Brain Mapp. 40, 1184-1194. https://doi.org/10.1002/hbm.24438

Jaramillo, S., Zador, A.M., 2011. The auditory cortex mediates the perceptual effects of acoustic temporal expectation, in: Nature Neuroscience. Nat Neurosci, pp. 246253. https://doi.org/10.1038/nn.2688

Jones, C.K., Byun, N., Bubser, M., 2012. Muscarinic and nicotinic acetylcholine receptor agonists and allosteric modulators for the treatment of schizophrenia. Neuropsychopharmacology. https://doi.org/10.1038/npp.2011.199

Kamke, M.R., Brown, M., Irvine, D.R.F., 2005. Basal forebrain cholinergic input is not essential for lesion-induced plasticity in mature auditory cortex. Neuron 48, 675686. https://doi.org/10.1016/j.neuron.2005.09.014

Kato, H.K., Gillet, S.N., Isaacson, J.S., 2015. Flexible Sensory Representations in Auditory Cortex Driven by Behavioral Relevance. Neuron 88, 1027-1039. https://doi.org/10.1016/j.neuron.2015.10.024

Kawaguchi, Y., Kondo, S., 2002. Parvalbumin, somatostatin and cholecystokinin as chemical markers for specific GABAergic interneuron types in the rat frontal cortex. J. Neurocytol. 31, 277-287. https://doi.org/10.1023/A:1024126110356

Keller, G.B., Mrsic-Flogel, T.D., 2018. Predictive Processing: A Canonical Cortical Computation. Neuron. https://doi.org/10.1016/j.neuron.2018.10.003 
Kilgard, M.P., Merzenich, M.M., 1998. Cortical map reorganization enabled by nucleus basalis activity. Science (80-. ). 279, 1714-1718.

https://doi.org/10.1126/science.279.5357.1714

King, A.J., Dahmen, J.C., Keating, P., Leach, N.D., Nodal, F.R., Bajo, V.M., 2011. Neural circuits underlying adaptation and learning in the perception of auditory space. Neurosci. Biobehav. Rev. https://doi.org/10.1016/j.neubiorev.2011.03.008

Klinkenberg, I., Blokland, A., Riedel, W.J., Sambeth, A., 2013. Cholinergic modulation of auditory processing, sensory gating and novelty detection in human participants. Psychopharmacology (Berl). https://doi.org/10.1007/s00213-012-2872-0

Kostal, L., Lansky, P., Stiber, M., 2018. Statistics of inverse interspike intervals: The instantaneous firing rate revisited. Chaos $28,1-8$. https://doi.org/10.1063/1.5036831

Kremláček, J., Kreegipuu, K., Tales, A., Astikainen, P., Põldver, N., Näätänen, R., Stefanics, G., 2016. Visual mismatch negativity (vMMN): A review and metaanalysis of studies in psychiatric and neurological disorders. Cortex. https://doi.org/10.1016/j.cortex.2016.03.017

Kuhn, J., Hardenacke, K., Lenartz, D., Gruendler, T., Ullsperger, M., Bartsch, C., Mai, J.K., Zilles, K., Bauer, A., Matusch, A., Schulz, R.J., Noreik, M., Bührle, C.P., Maintz, D., Woopen, C., Häussermann, P., Hellmich, M., Klosterkötter, J., Wiltfang, J., Maarouf, M., Freund, H.J., Sturm, V., 2015. Deep brain stimulation of the nucleus basalis of Meynert in Alzheimer's dementia. Mol. Psychiatry. https://doi.org/10.1038/mp.2014.32

Kuhn, Jens, Hardenacke, K., Shubina, E., Lenartz, D., Visser-Vandewalle, V., Zilles, K., Sturm, V., Freund, H.J., 2015. Deep brain stimulation of the nucleus basalis of meynert in early stage of Alzheimer's dementia. Brain Stimul. https://doi.org/10.1016/j.brs.2015.04.002

Kumbhare, D., Palys, V., Toms, J., Wickramasinghe, C.S., Amarasinghe, K., Manic, M., Hughes, E., Holloway, K.L., 2018. Nucleus basalis of Meynert stimulation for dementia: Theoretical and technical considerations. Front. Neurosci. https://doi.org/10.3389/fnins.2018.00614

Lee, C.C., Winer, J.A., 2008. Connections of cat auditory cortex: I. Thalamocortical system. J. Comp. Neurol. https://doi.org/10.1002/cne.21611

Liljenstrom, H., Hasselmo, M.E., 1995. Cholinergic modulation of cortical oscillatory dynamics. J. Neurophysiol. 74, 288-297. https://doi.org/10.1152/jn.1995.74.1.288

Loewi, O., 1921. Über humorale übertragbarkeit der Herznervenwirkung - I. Mitteilung. Pflugers Arch. Gesamte Physiol. Menschen Tiere 189, 239-242. https://doi.org/10.1007/BF01738910

Lucas-Meunier, E., Fossier, P., Baux, G., Amar, M., 2003. Cholinergic modulation of the cortical neuronal network. Pflugers Arch. Eur. J. Physiol. https://doi.org/10.1007/s00424-002-0999-2

Ma, X., Suga, N., 2005. Long-term cortical plasticity evoked by electric stimulation and acetylcholine applied to the auditory cortex. Proc. Natl. Acad. Sci. U. S. A. 102, 9335-9340. https://doi.org/10.1073/pnas.0503851102

Malmierca, M.S., 2015. Auditory System, in: The Rat Nervous System: Fourth Edition. https://doi.org/10.1016/B978-0-12-374245-2.00029-2

Malmierca, Manuel S., Cristaudo, S., Pérez-González, D., Covey, E., 2009. Stimulusspecific adaptation in the inferior colliculus of the anesthetized rat. J. Neurosci. 29, 5483-5493. https://doi.org/10.1523/JNEUROSCI.4153-08.2009

Malmierca, Manuel S, Cristaudo, S., Pérez-González, D., Covey, E., 2009. Behavioral/Systems/Cognitive Stimulus-Specific Adaptation in the Inferior 
Colliculus of the Anesthetized Rat. Soc Neurosci.

https://doi.org/10.1523/JNEUROSCI.4153-08.2009

Malmierca, M.S., Sanchez-Vives, M. V., Escera, C., Bendixen, A., 2014. Neuronal adaptation, novelty detection and regularity encoding in audition. Front. Syst. Neurosci. https://doi.org/10.3389/fnsys.2014.00111

Mansour, S., Magnan, J., Haidar, H., Nicolas, K., Louryan, S., Mansour, S., Magnan, J., Haidar, H., Nicolas, K., Louryan, S., 2013. Middle Ear Contents, in:

Comprehensive and Clinical Anatomy of the Middle Ear. Springer Berlin Heidelberg, pp. 49-81. https://doi.org/10.1007/978-3-642-36967-4_3

Marshall, L., Mathys, C., Ruge, D., de Berker, A.O., Dayan, P., Stephan, K.E., Bestmann, S., 2016. Pharmacological Fingerprints of Contextual Uncertainty. PLoS Biol. 14. https://doi.org/10.1371/journal.pbio.1002575

Martins, A.R.O., Froemke, R.C., 2015. Coordinated forms of noradrenergic plasticity in the locus coeruleus and primary auditory cortex. Nat. Neurosci. 18, 1483-1492. https://doi.org/10.1038/nn.4090

Matthews, N., Todd, J., Mannion, D.J., Finnigan, S., Catts, S., Michie, P.T., 2013. Impaired processing of binaural temporal cues to auditory scene analysis in schizophrenia. Schizophr. Res. 146, 344-348. https://doi.org/10.1016/j.schres.2013.02.013

May, P., Tiitinen, H., Ilmoniemi, R.J., Nyman, G., Taylor, J.G., Näätänen, R., 1999. Frequency change detection in human auditory cortex. J. Comput. Neurosci. https://doi.org/10.1023/A:1008896417606

Mendoza, G., Peyrache, A., Gámez, J., Prado, L., Buzsáki, G., Merchant, H., 2016. Recording extracellular neural activity in the behaving monkey using a semichronic and high-density electrode system. J. Neurophysiol. 116, 563-574. https://doi.org/10.1152/jn.00116.2016

Mesulam, M.M., 2013. Cholinergic circuitry of the human nucleus basalis and its fate in Alzheimer's disease. J. Comp. Neurol. https://doi.org/10.1002/cne.23415

Metherate, R., 2011. Functional connectivity and cholinergic modulation in auditory cortex. Neurosci. Biobehav. Rev. https://doi.org/10.1016/j.neubiorev.2010.11.010

Metherate, R., Cox, C.L., Ashe, J.H., 1992. Cellular bases of neocortical activation: Modulation of neural oscillations by the nucleus basalis and endogenous acetylcholine. J. Neurosci. 12, 4701-4711. https://doi.org/10.1523/jneurosci.12-1204701.1992

Michalareas, G., Vezoli, J., van Pelt, S., Schoffelen, J.M., Kennedy, H., Fries, P., 2016. Alpha-Beta and Gamma Rhythms Subserve Feedback and Feedforward Influences among Human Visual Cortical Areas. Neuron 89, 384-397. https://doi.org/10.1016/j.neuron.2015.12.018

Moore, A.K., Wehr, M., 2013. Parvalbumin-Expressing Inhibitory Interneurons in Auditory Cortex Are Well-Tuned for Frequency. J. Neurosci. 33, 13713-13723. https://doi.org/10.1523/JNEUROSCI.0663-13.2013

Moran, R.J., Campo, P., Symmonds, M., Stephan, K.E., Dolan, R.J., Friston, K.J., 2013. Behavioral/Cognitive Free Energy, Precision and Learning: The Role of Cholinergic Neuromodulation. Soc Neurosci. https://doi.org/10.1523/JNEUROSCI.4255-12.2013

Motts, S.D., Schofield, B.R., 2009. Sources of cholinergic input to the inferior colliculus. Neuroscience 160, 103-114. https://doi.org/10.1016/j.neuroscience.2009.02.036

Mountcastle, V.B., Talbot, W.H., Sakata, H., Hyvärinen, J., 1969. Cortical neuronal mechanisms in flutter-vibration studied in unanesthetized monkeys. Neuronal 
periodicity and frequency discrimination. J. Neurophysiol. 32, 452-484.

https://doi.org/10.1152/jn.1969.32.3.452

Muñoz, W., Rudy, B., 2014. Spatiotemporal specificity in cholinergic control of neocortical function. Curr. Opin. Neurobiol. https://doi.org/10.1016/j.conb.2014.02.015

Muñoz, W., Tremblay, R., Levenstein, D., Rudy, B., 2017. Layer-specific modulation of neocortical dendritic inhibition during active wakefulness. Science (80-. ). 355, 954-959. https://doi.org/10.1126/science.aag2599

Näätänen, R., 2018. Attention and brain function, Attention and Brain Function. https://doi.org/10.4324/9780429487354

Näätänen, R., 2009. Somatosensory mismatch negativity: A new clinical tool for developmental neurological research? Dev. Med. Child Neurol. https://doi.org/10.1111/j.1469-8749.2009.03386.x

Näätänen, R., Gaillard, A.W.K., Mäntysalo, S., 1978. Early selective-attention effect on evoked potential reinterpreted. Acta Psychol. (Amst). https://doi.org/10.1016/00016918(78)90006-9

Näätänen, R., Paavilainen, P., Rinne, T., Alho, K., 2007. The mismatch negativity $(\mathrm{MMN})$ in basic research of central auditory processing: A review. Clin. Neurophysiol. https://doi.org/10.1016/j.clinph.2007.04.026

Näätänen, R., Tervaniemi, M., Sussman, E., Paavilainen, P., Winkler, I., 2001. "Primitive intelligence" in the auditory cortex. Trends Neurosci. https://doi.org/10.1016/S0166-2236(00)01790-2

Näätänen, R., Winkler, I., 1999. The concept of auditory stimulus representation in cognitive neuroscience. Psychol. Bull. https://doi.org/10.1037/00332909.125.6.826

Natan, R.G., Briguglio, J.J., Mwilambwe-Tshilobo, L., Jones, S.I., Aizenberg, M., Goldberg, E.M., Geffen, M.N., 2015. Complementary control of sensory adaptation by two types of cortical interneurons. Elife 4 . https://doi.org/10.7554/eLife.09868

Nelken, I., 2014. Stimulus-specific adaptation and deviance detection in the auditory system: experiments and models. Biol. Cybern. https://doi.org/10.1007/s00422014-0585-7

Netser, S., Zahar, Y., Gutfreund, Y., 2011. Stimulus-specific adaptation: Can it be a neural correlate of behavioral habituation? J. Neurosci. 31, 17811-17820. https://doi.org/10.1523/JNEUROSCI.4790-11.2011

Newman, E.L., Gupta, K., Climer, J.R., Monaghan, C.K., Hasselmo, M.E., 2012. Cholinergic modulation of cognitive processing: Insights drawn from computational models. Front. Behav. Neurosci. https://doi.org/10.3389/fnbeh.2012.00024

Nieto-Diego, J., Malmierca, M.S., 2016. Topographic Distribution of Stimulus-Specific Adaptation across Auditory Cortical Fields in the Anesthetized Rat. PLoS Biol. 14. https://doi.org/10.1371/journal.pbio.1002397

Noudoost, B., Moore, T., 2011. The role of neuromodulators in selective attention. Trends Cogn. Sci. https://doi.org/10.1016/j.tics.2011.10.006

Ogawa, H., Oka, K., 2015. Direction-Specific Adaptation in Neuronal and Behavioral Responses of an Insect Mechanosensory System. Soc Neurosci. https://doi.org/10.1523/JNEUROSCI.1378-15.2015

Parr, T., Friston, K.J., 2017. Uncertainty, epistemics and active inference. royalsocietypublishing.org 14. https://doi.org/10.1098/rsif.2017.0376

Parras, G.G., Nieto-Diego, J., Carbajal, G. V., Valdés-Baizabal, C., Escera, C., 
Malmierca, M.S., 2017a. Neurons along the auditory pathway exhibit a hierarchical organization of prediction error. Nat. Commun. 8.

https://doi.org/10.1038/s41467-017-02038-6

Parras, G.G., Nieto-Diego, J., Carbajal, G. V., Valdés-Baizabal, C., Escera, C., Malmierca, M.S., 2017b. Neurons along the auditory pathway exhibit a hierarchical organization of prediction error. Nat. Commun. 8. https://doi.org/10.1038/s41467-017-02038-6

Paxinos, G., Watson, C., Diego, S., Boston, L., York, N., 1997. The Rat Brainin Stereotaxic Coordinates Academic Press.

Pérez-González, D., Hernández, O., Covey, E., Malmierca, M.S., 2012. GABA(A)mediated inhibition modulates stimulus-specific adaptation in the inferior colliculus. PLoS One 7, e34297. https://doi.org/10.1371/journal.pone.0034297

Pérez-González, D., Malmierca, M.S., 2014. Adaptation in the auditory system: An overview. Front. Integr. Neurosci. https://doi.org/10.3389/fnint.2014.00019

Pérez-González, D., Malmierca, M.S., 2012. Variability of the time course of stimulusspecific adaptation in the inferior colliculus. Front. Neural Circuits 6. https://doi.org/10.3389/fncir.2012.00107

Pérez-González, D., Malmierca, M.S., Covey, E., 2005. Novelty detector neurons in the mammalian auditory midbrain. Eur. J. Neurosci. https://doi.org/10.1111/j.14609568.2005.04472.x

Peyrache, A., Dehghani, N., Eskandar, E.N., Madsen, J.R., Anderson, W.S., Donoghue, J.A., Hochberg, L.R., Halgren, E., Cash, S.S., Destexhe, A., 2012. Spatiotemporal dynamics of neocortical excitation and inhibition during human sleep. Proc. Natl. Acad. Sci. U. S. A. 109, 1731-1736. https://doi.org/10.1073/pnas.1109895109

Picciotto, M.R., Higley, M.J., Mineur, Y.S., 2012. Acetylcholine as a Neuromodulator: Cholinergic Signaling Shapes Nervous System Function and Behavior. Neuron. https://doi.org/10.1016/j.neuron.2012.08.036

Pickles, J.O., 2015. Auditory pathways: Anatomy and physiology, in: Handbook of Clinical Neurology. https://doi.org/10.1016/B978-0-444-62630-1.00001-9

Pinto, L., Goard, M.J., Estandian, D., Xu, M., Kwan, A.C., Lee, S.H., Harrison, T.C., Feng, G., Dan, Y., 2013. Fast modulation of visual perception by basal forebrain cholinergic neurons. Nat. Neurosci. 16, 1857-1863. https://doi.org/10.1038/nn.3552

Polack, P.O., Friedman, J., Golshani, P., 2013. Cellular mechanisms of brain statedependent gain modulation in visual cortex. Nat. Neurosci. 16, 1331-1339. https://doi.org/10.1038/nn.3464

Polley, D.B., Read, H.L., Storace, D.A., Merzenich, M.M., 2007. Multiparametric auditory receptive field organization across five cortical fields in the albino rat. J. Neurophysiol. 97, 3621-3638. https://doi.org/10.1152/jn.01298.2006

Popelar, J., Groh, D., Pelánová, J., Canlon, B., Syka, J., 2006. Age-related changes in cochlear and brainstem auditory functions in Fischer 344 rats. Neurobiol. Aging 27, 490-500. https://doi.org/10.1016/j.neurobiolaging.2005.03.001

Profant, O., Burianová, J., Syka, J., 2013. The response properties of neurons in different fields of the auditory cortex in the rat. Hear. Res. https://doi.org/10.1016/j.heares.2012.11.021

Puckett, A.C., Pandya, P.K., Moucha, R., Dai, W., Kilgard, M.P., 2007. Plasticity in the Rat Posterior Auditory Field Following Nucleus Basalis Stimulation. J. Neurophysiol. 98, 253-265. https://doi.org/10.1152/jn.01309.2006

Recanzone, G.H., Schreiner, C.E., Merzenich, M.M., 1993. Plasticity in the frequency representation of primary auditory cortex following discrimination training in adult 
owl monkeys. J. Neurosci. 13, 87-103. https://doi.org/10.1523/jneurosci.13-0100087.1993

Recasens, M., Gross, J., Uhlhaas, P.J., 2018. Low-Frequency Oscillatory Correlates of Auditory Predictive Processing in Cortical-Subcortical Networks: A MEG-Study. Sci. Rep. 8, 14007. https://doi.org/10.1038/s41598-018-32385-3

Ruhnau, P., Herrmann, B., Schröger, E., 2012. Finding the right control: The mismatch negativity under investigation. Clin. Neurophysiol. 123, 507-512. https://doi.org/10.1016/j.clinph.2011.07.035

Ruzzoli, M., Pirulli, C., Mazza, V., Miniussi, C., Brignani, D., 2016. The mismatch negativity as an index of cognitive decline for the early detection of Alzheimer's disease. Sci. Rep. 6, 1-11. https://doi.org/10.1038/srep33167

Safieddine, S., El-Amraoui, A., Petit, C., 2012. The Auditory Hair Cell Ribbon Synapse: From Assembly to Function. Annu. Rev. Neurosci. https://doi.org/10.1146/annurev-neuro-061010-113705

Saldaña, E., 2015. All the Way from the Cortex: a Review of Auditory Corticosubcollicular Pathways. Cerebellum. https://doi.org/10.1007/s12311-0150694-4

Sarter, M., Givens, B., Bruno, J.P., 2001. The cognitive neuroscience of sustained attention: Where top-down meets bottom-up. Brain Res. Rev. https://doi.org/10.1016/S0165-0173(01)00044-3

Sceniak, M.P., MacIver, M.B., 2006. Cellular actions of urethane on rat visual cortical neurons in vitro. J. Neurophysiol. 95, 3865-3874. https://doi.org/10.1152/jn.01196.2005

Schofield, B.R., 2011. Central Descending Auditory Pathways. https://doi.org/10.1007/978-1-4419-7070-1_9

Schofield, B.R., Coomes, D.L., 2005. Projections from auditory cortex contact cells in the cochlear nucleus that project to the inferior colliculus. Hear. Res. https://doi.org/10.1016/j.heares.2005.03.005

Schreiner, C.E., Polley, D.B., 2014. Auditory map plasticity: Diversity in causes and consequences. Curr. Opin. Neurobiol. https://doi.org/10.1016/j.conb.2013.11.009

Schröger, E., Wolff, C., 1996. Mismatch response of the human brain to changes in sound location. Neuroreport 7, 3005-3008. https://doi.org/10.1097/00001756199611250-00041

Sedley, W., Gander, P.E., Kumar, S., Kovach, C.K., Oya, H., Kawasaki, H., Howard, M.A., Griffiths, T.D., 2016. Neural signatures of perceptual inference. Elife 5. https://doi.org/10.7554/eLife.11476

Shen, G., Weiss, S.M., Meltzoff, A.N., Marshall, P.J., 2018. The somatosensory mismatch negativity as a window into body representations in infancy. Int. J. Psychophysiol. 134, 144-150. https://doi.org/10.1016/j.ijpsycho.2018.10.013

Shiramatsu, T.I., Kanzaki, R., Takahashi, H., 2013. Cortical mapping of mismatch negativity with deviance detection property in rat. PLoS One. https://doi.org/10.1371/journal.pone.0082663

Sikkens, T., Bosman, C.A., Olcese, U., 2019. The Role of Top-Down Modulation in Shaping Sensory Processing Across Brain States: Implications for Consciousness. Front. Syst. Neurosci. https://doi.org/10.3389/fnsys.2019.00031

Soma, S., Shimegi, S., Suematsu, N., Sato, H., 2013. Cholinergic modulation of response gain in the rat primary visual cortex. Sci. Rep. https://doi.org/10.1038/srep01138

Stefanics, G., Astikainen, P., Czigler, I., 2015. Visual mismatch negativity (vMMN): a prediction error signal in the visual modality. Front. Hum. Neurosci. 8, 1074. 
https://doi.org/10.3389/fnhum.2014.01074

Stefanics, G., Stefanics, G., Kremláček, J., Czigler, I., 2014. Visual mismatch negativity: A predictive coding view. Front. Hum. Neurosci. https://doi.org/10.3389/fnhum.2014.00666

Summerfield, C., Trittschuh, E.H., Monti, J.M., Mesulam, M.M., Egner, T., 2008. Neural repetition suppression reflects fulfilled perceptual expectations. Nat. Neurosci. 11, 1004-1006. https://doi.org/10.1038/nn.2163

Sussman, E., Winkler, I., 2001. Dynamic sensory updating in the auditory system. Cogn. Brain Res. https://doi.org/10.1016/S0926-6410(01)00067-2

Szabó, B.T., Denham, S.L., Winkler, I., 2016. Computational models of auditory scene analysis: A review. Front. Neurosci. https://doi.org/10.3389/fnins.2016.00524

Thiele, A., Bellgrove, M.A., 2018. Neuromodulation of Attention. Neuron. https://doi.org/10.1016/j.neuron.2018.01.008

Todorovic, A., van Ede, F., Maris, E., de Lange, F.P., 2011. Prior expectation mediates neural adaptation to repeated sounds in the auditory cortex: An MEG study. J. Neurosci. 31, 9118-9123. https://doi.org/10.1523/JNEUROSCI.1425-11.2011

Tremblay, R., Lee, S., Rudy, B., 2016. GABAergic Interneurons in the Neocortex: From Cellular Properties to Circuits. Neuron. https://doi.org/10.1016/j.neuron.2016.06.033

Ulanovsky, N., Las, L., Farkas, D., Nelken, I., 2005. “ Multiple time scales of adaptation in auditory cortex neurons": Correction.

Ulanovsky, N., Las, L., Nelken, I., 2003. Processing of low-probability sounds by cortical neurons. Nat. Neurosci. 6, 391-398. https://doi.org/10.1038/nn1032

Valdés-Baizabal, C., Carbajal, G. V., Pérez-González, D., Malmierca, M.S., 2020a. Dopamine gates prediction error forwarding in the cortices of the inferior colliculus. PLoS Biol. https://doi.org/10.1101/824656

Valdés-Baizabal, C., Carbajal, G. V., Pérez-González, D., Malmierca, M.S., 2020b. Dopamine modulates subcortical responses to surprising sounds. PLoS Biol. 18, e3000744. https://doi.org/10.1371/journal.pbio.3000744

Valdés-Baizabal, C., Carbajal, G. V., Pérez-González, D., Malmierca, M.S., 2019. Dopamine gates prediction error forwarding in the cortices of the inferior colliculus. bioRxiv 824656. https://doi.org/10.1101/824656

Veit, J., Hakim, R., Jadi, M.P., Sejnowski, T.J., Adesnik, H., 2017. Cortical gamma band synchronization through somatostatin interneurons. Nat. Neurosci. 20, 951959. https://doi.org/10.1038/nn.4562

Villano, I., Messina, A., Valenzano, A., Moscatelli, F., Esposito, T., Monda, V., Esposito, M., Precenzano, F., Carotenuto, M., Viggiano, A., Chieffi, S., Cibelli, G., Monda, M., Messina, G., 2017. Basal forebrain cholinergic system and orexin neurons: Effects on attention. Front. Behav. Neurosci. https://doi.org/10.3389/fnbeh.2017.00010

Von Der Behrens, W., Bäuerle, P., Kössl, M., Gaese, B.H., 2009. Behavioral/Systems/Cognitive Correlating Stimulus-Specific Adaptation of Cortical Neurons and Local Field Potentials in the Awake Rat. Soc Neurosci. https://doi.org/10.1523/JNEUROSCI.3475-09.2009

Vossel, S., Weidner, R., Driver, J., Friston, K.J., Fink, G.R., 2012. Deconstructing the architecture of dorsal and ventral attention systems with dynamic causal modeling. J. Neurosci. 32, 10637-10648. https://doi.org/10.1523/JNEUROSCI.0414-12.2012

Weinberger, N.M., 2007. Auditory associative memory and representational plasticity in the primary auditory cortex. Hear. Res. https://doi.org/10.1016/j.heares.2007.01.004 
Weinberger, N.M., 2004. Specific long-term memory traces in primary auditory cortex. Nat. Rev. Neurosci. 5, 279-290. https://doi.org/10.1038/nrn1366

Winer, J.A., Prieto, J.J., 2001. Layer V in cat primary auditory cortex (AI): Cellular architecture and identification of projection neurons. J. Comp. Neurol. https://doi.org/10.1002/cne.1183

Winkler, I., Karmos, G., Näätänen, R., 1996. Adaptive modeling of the unattended acoustic environment reflected in the mismatch negativity event-related potential. Brain Res. https://doi.org/10.1016/S0006-8993(96)01008-6

Wood, K.C., Blackwell, J.M., Geffen, M.N., 2017. Cortical inhibitory interneurons control sensory processing. Curr. Opin. Neurobiol. https://doi.org/10.1016/j.conb.2017.08.018

Xu, H., Jeong, H.Y., Tremblay, R., Rudy, B., 2013. Neocortical SomatostatinExpressing GABAergic Interneurons Disinhibit the Thalamorecipient Layer 4. Neuron 77, 155-167. https://doi.org/10.1016/j.neuron.2012.11.004

Yabe, H., Tervaniemi, M., Reinikainen, K., Näätänen, R., 1997. Temporal window of integration revealed by MMN to sound omission. Neuroreport. https://doi.org/10.1097/00001756-199705260-00035

Yaron, A., Jankowski, M.M., Badrieh, R., Nelken, I., 2020. Stimulus-specific adaptation to behaviorally-relevant sounds in awake rats. PLoS One. https://doi.org/10.1371/journal.pone.0221541

Yu, A.J., Dayan, P., 2005. Uncertainty, neuromodulation, and attention. Neuron 46, 681-692. https://doi.org/10.1016/j.neuron.2005.04.026

Yu, A.J., Dayan, P., 2002. Acetylcholine in cortical inference. Neural Networks 15, 719-730. https://doi.org/10.1016/S0893-6080(02)00058-8

Zaborszky, L., Hoemke, L., Mohlberg, H., Schleicher, A., Amunts, K., Zilles, K., 2008. Stereotaxic probabilistic maps of the magnocellular cell groups in human basal forebrain. Neuroimage 42, 1127-1141. https://doi.org/10.1016/j.neuroimage.2008.05.055

Znamenskiy, P., Zador, A.M., 2013. Corticostriatal neurons in auditory cortex drive decisions during auditory discrimination. Nature 497, 482-485. https://doi.org/10.1038/nature12077 


\section{SUPPLEMENTARY FIGURES}

EXPERIMENTAL CONDITIONS A


CONTROL CONDITIONS

\section{B}
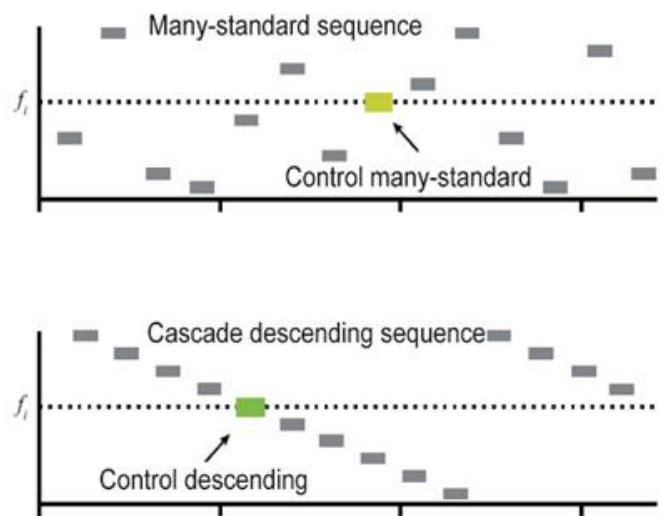

Cascade ascending sequence

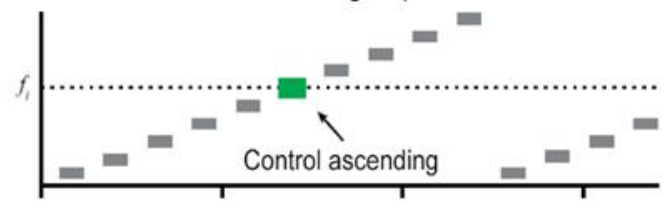

C PREDICTIVE CODING HYPOTHESIS

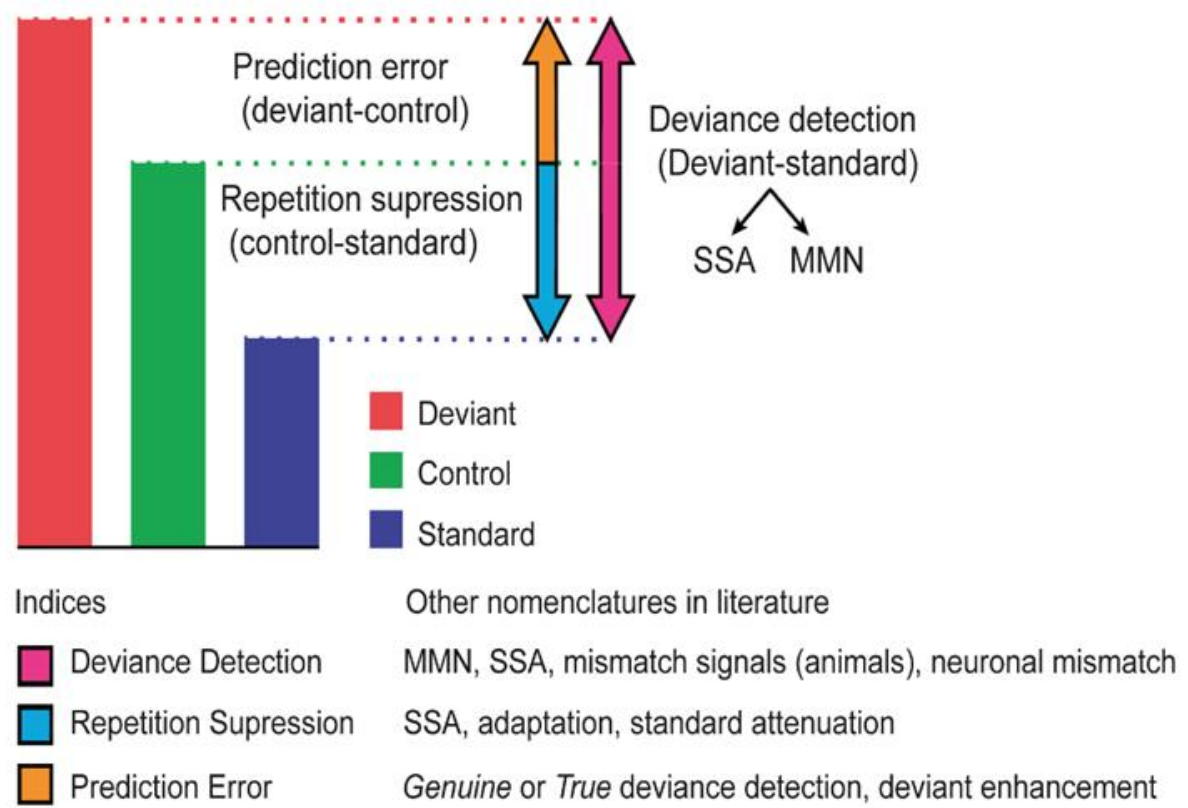




\section{Supplementary Figure 1. Experimental paradigms and Interpretation of}

the control conditions. (A) Classic oddball paradigm, showing three possible experimental conditions for a given $f_{i}$ target tone: STD (blue circle, top panel), DEV descending (red triangle pointing downwards, middle panel) and DEV ascending (red triangle pointing upwards, bottom panel). (B) CTR sequences highlighting the $f_{i}$ target tone. In the many-standard control (very light green rectangle), the target tone is embedded within a random succession of equiprobable tones, making impossible for the system to establish a precise prediction or accurately encode a regularity. Two versions of the cascade sequence: Cascade descending sequence (light green rectangle) and Cascade ascending sequence (dark green rectangle) are compared with the DEV descending and ascending, respectively. In both versions, the target tone $f_{i}$ is embedded in a regular succession of equiprobable tones in both cases. This allows the system to generate a precise prediction or encode that regularity, because it is not violated by the appearance of the target tone $f_{i}$, as opposed to DEV. (C) Decomposition of the differential response by means of the control condition (green bar), yielding indices of mismatch (iMM, in magenta), prediction error (iPE, in orange) and repetition suppression (iRS, in cyan). Modified after (G. V Carbajal and Malmierca, 2018; Guillermo V. Carbajal and Manuel S. Malmierca, 2020). 
Dear Dr Malmierca,

Thank you for submitting your article "The Role of Cholinergic Neuromodulation in Forwarding Prediction Error in the Auditory Cortex" for consideration by eLife. Your article has been reviewed by 3 peer reviewers, and the evaluation has been overseen by a Reviewing Editor and Barbara Shinn-Cunningham as the Senior Editor. The following individuals involved in review of your submission have agreed to reveal their identity: Hugo Merchant (Reviewer \#1); Rosalyn Moran (Reviewer \#2).

The reviewers have discussed the reviews with one another and the Reviewing Editor has drafted this decision to help you prepare a revised submission.

We would like to draw your attention to changes in our revision policy that we have made in response to COVID-19 (https://elifesciences.org/articles/57162). Specifically, when editors judge that a submitted work as a whole belongs in eLife but that some conclusions require a modest amount of additional new data, as they do with your paper, we are asking that the manuscript be revised to either limit claims to those supported by data in hand, or to explicitly state that the relevant conclusions require additional supporting data. Our expectation is that the authors will eventually carry out the additional experiments and report on how they affect the relevant conclusions either in a preprint on bioRxiv or medRxiv, or if appropriate, as a Research Advance in eLife, either of which would be linked to the original paper.

Our reviewers agree that more data mining is needed to further characterize the role of Acetylcholine (Ach) in the increased responses to the deviant stimulus. These analyses should focus on response parameters beyond the changes in discharge rate (onset latency, duration peak response), discard any unspecific effect of Ach improving general signal detectability, and further examine the Ach effects within the auditory cortical hierarchy and laminar specificity. Please see the enclosed reviews.

\section{1) SUMMARY:}

In their manuscript the authors describe the effects of cholinergic manipulation on neuronal firing rates in response to auditory stimuli sequences using classical and controlled mismatch paradigms. They explore the role of acetylcholine on altering the firing rate properties across different hierarchical levels of auditory cortex and in different layers of $A C$ regions. With a rigorous analysis they demonstrate that Ach primarily mediates firing rate responses to deviant tones. They present their results within the context of predictive coding theories of sensory perception - concluding an effect on prediction error precision. Moreover, they use two specific receptor modulations to demonstrate that the effects are mediated by muscarinic and not nicotinic cholinergic receptors. The findings are clear and well presented and the claims justified, the manuscript represents a strong contribution to the field of neuromodulation, dissecting ACh's role in primary sensory signal trafficking.

\section{2) REVISIONS FOR THIS PAPER:}

\section{Main Comments.}

The authors use the predictive coding framework to explain their findings. However, the conclusion of the paper is not well supported by the findings of the paper in the present form. The authors did not measure the precision of prediction error signaling, they 
measured the number of spikes in three stimulation conditions and compute indexes as a tool to test their hypothesis. Consequently, this paper will strongly benefit for a more comprehensive analysis on the neural responses. Specifically, here are the reviewers' recommendations on the analysis of the data:

A. Besides the discharge rate, it is critical to compute the response onset latency, the duration of the activation periods and the intertrial variability of the activation period in the control, Ach, and recovery for the three stimulus conditions. The increase in number of spikes in the $\mathrm{ACH}$ deviant condition can be associated with changes the mean and variability of duration, activation peak and onset latency of the activation periods. Importantly, with the intertrial variability of these measures is possible to directly test whether it is the precision of the prediction error signal what is affected by $\mathrm{ACH}$. Then, the paper should use ANOVAS and regression models to determine the effect of Ach on the three stimulus conditions across the dependent variables described above.

B. I suggest to also employ other metrics such as Mutual Information or ROC analysis to determine how much the response distributions for deviant and standard stimulus overlap before and after acetylcholine injections and how well the stimulus identity can be decoded from the neuronal firing rate. My suggestion is also driven by the fact that the indices employed in the study can be misleading since they only reflect the spike count response (mean spike/trial) but do not take into account the response probability of the neuron, i.e., how many trials the neurons responded to. Likewise, it is important to note that acetylcholine can affect the general neural responsiveness without affecting the SSA indices or to exert a strong effect in the deviant response but almost without changing the SSA index (as observed in Fig 3, VAF example).

C. Please clarify some of the broader statistical methods with respect to animal numbers and the responsive cell counts. A total of 122 units were used for the main analysis. In the methods it states that 30 animals were used with a tungsten electrode per animal? How were the units identified? Were different regions measured from different animals?

D. Please consider that the excellent data could be mined a little further to examine the auditory cortical hierarchy or laminar specificity.

E. My main concern relates to the specificity of the findings. With the current analyses, I found it difficult to discard any unspecific effect of Ach improving general signal detectability. To assess this problem, the authors should show PSTHs of neurons under the three stimulation protocols (cascade, standards, and deviant) during the control and the Ach conditions. The authors already show violin plots of cascade stimulation responses in Figure 6, but a figure similar to Figure 2, including the cascade response, would be much more informative.

$\mathrm{F}$. The authors show wide variability on the CSI changes related to the administration of Ach (Figure 3) along the lemniscal pathway. Further, the examples of Figure 2 seem to indicate the activity of more than one neuronal population involved in deviant detection. However, no further analyses of these observations were carried out. The authors could show whether this variability is related to any specific neuronal population correlating the CSI values with any response parameter (hopefully waveforms) of the observed neurons. This observation would help to establish how specific is the effect of Ach in DD responses and clarify the differential response to Ach from $A C$ and IC neurons.

G. More advanced analyses could also be considered such as testing for hierarchical arrangements within these data - for example, in the analysis of basal forebrain firing rates: $\underline{\text { https://doi.org/10.3389/fnbeh.2015.00277 }}$ 
Regarding the Discussion:

A. The discussion about oscillations and the role of Ach in hierarchical communication is very speculative. The authors could bring support to their model including some analyses of the LFP responses observed at different layers, exploring whether changes in high and low-frequency band power correlates with these observations.

B. Please consider a more direct discussion, contrasting the prediction coding scheme with the alternative possibility of changes input gain and local processing as a mechanism behind the increase in deviant responses by Ach. Also consider that the modulatory effects observed in anesthetized animals are hard to compare with studies in awake behaving subjects.

C. Previous studies have shown Ach enhancements of the neuronal activity in V1 in monkeys performing an attentional task (Herrero et al. 2008 Nature 454:1110). These results strongly resemble the findings of this study. Ach increases the response of attentive neurons within the RF (but not outside), and Muscarinic receptors mediate this effect. The authors should discuss how the effects on deviant detection enhancement are related to these attentional effects, assessing the question about how much specific is the effect of Ach on prediction.

\section{MINOR POINTS:}

The paper has way too many abbreviations, it is difficult to follow, unless you are very familiar with the terminology, which will not be necessarily the case for an eLife paper. Related, Figure 1B is a little confusing - this isn't the firing rate but the response profile according to stimulus frequency? Are all recording electrodes contributing equal numbers to the total unit count - or are some regions more represented in terms of 'active units'?

Question: Can the larger response to the deviant in the oddball sequence with respect to the same tone in the cascade sequences be the result of a generalized decrease in the neural responsiveness under the cascade condition?

The authors should explain in the results that the indexes iMM, iPE and iRS measure. It is difficult to understand the rationale of merging neurons from all cortical regions together (Fig. 4) when previous studies have clearly demonstrated that SSA level and discharge rates varies among cortical auditory areas and that acetylcholine exerted a differential modulation on neurons with variable level of SSA in other auditory areas. Likewise, authors claimed top-down and bottom up signals can be affecting the SSA indices (L81-92). Then, authors should explore the interaction between the layer (supra-, infragranular) and cortical area (lemniscal and non lemniscal) factors following feedforward and feedback models of cortical connectivity. Instead of that, authors merge all data together (Fig. 5D-E) and did not described how the magnitude of cholinergic modulation changes across cortical areas but only reported pair-wise comparison (Fig. 4D, 5E). In my opinion, it should be first determined how the repetition suppression and prediction error changes along the auditory cortical hierarchy and later to determine how acetylcholine modifies that pattern. By doing that, authors can gain more insights on how sound features are transformed along the auditory hierarchy and modulated by local inputs. Finally, it is not clear whether the number of studied neurons is enough to determine topographical differences between subareas and cortical layers.

In the methods the CSI is defined as $d(f 1)+d f(2)$... how are $d(f 1)$ and $d(f 2)$ computed rates per electrode or per unit. Did the authors examine the tonotopy to select the frequencies for each particular animal? Was the firing rate calculated for tone presentation or for the whole interstimulus interval?

Were recordings where there was a response to the deviant but no response to the 
standard included in the analysis? In particular I am confused by the following paragraph where neuron selection is described:

Once a suitable neuron was found, the frequency response ...FRA) of the cell, i.e. the combination of frequencies and intensities capable of evoking a suprathreshold response, ...Based on this information, we selected a pair of frequencies evoking similar responses at 10-30 dB above threshold.

The drug analysis seems to be performed for a different subset of cells. Pg 5 the text mentions that from $n=99$, scopolamine $(n=13)$ and mecamylamine $(n=10)$ to test if the effects observed with Ach. Why are there only 13 and 10 units included in the drug data?

Figure 2 suggests that the application of Ach has a more punctate effect in A1 as compared to PAF for example. The analysis of region specific effects in figure 4 was an ANOVA applied to examine main effects of standard/deviants and interactions with region?

Similarly in figure 5, the layer specific results are collapsed across regions. It appears that they may be some preferential effect in layer IV, for example but this may be specific to A1 - but the specificity is not explored. Perhaps the number of data points is too small but would it be possible to apply again an analysis of variance to test for an interaction of layer and region (this would be a useful add-on in terms of the predictive coding framework).

Did ACh have any effect on the receptive field characteristics spatially - is it possible to test the 'sharpening' wrt the spatial extent (controlling for mean firing rate changes). FRA' not defined at first use (in results), CF' not defined at first use (in results) \& other acronyms (IC).

Figure 4 - how is the 95\% confidence interval in the CSI calculated? (since per trial analysis doesn't make sense in the context of an oddball sequence)

Figure 6, the cascade experiment should be signposted more clearly in the results section - i.e. what is one testing for here.

What is the take home from the correlation plots in figure $6 \mathrm{H}$ and $6 \mathrm{I}$ - aren't these metrics derived from similar baseline statistics anyway?

Figure 7 appears somewhat out of the blue - these data from the IC are not reported here but from a previous publication and the reader might be left wondering if they missed something!

* We will look forward to hearing from you with a revised article with tracked changes, and a response letter (uploaded as an editable file) describing the changes made in response to the decision and review comments.

* If source manuscript files have not already been provided, we will need them at the revision stage; further details are

here: https://submit.elifesciences.org/html/elife author instructions.html\#revised

* If your study includes bench research, please include a Key Resources Table within your resubmission. This is designed to highlight genetically modified organisms and strains, cell lines, reagents, and software that are essential to reproduce the results presented (but it is not designed to be a comprehensive list of all the materials and resources used).You should download the following template, which includes notes on 
completion as well as an example table: [https://elife-cdn.s3.amazonaws.com/authorguide/elifeKRTtemplatev2.docx] For relevant submissions, the completed table should be incorporated within your article file at the very beginning of the Materials and Methods section (examples in published articles are available at [https://elifesciences.org/articles/44199\#s4] and [https://elifesciences.org/articles/45342\#s4]).

* If your work involved the use of cell lines, please indicate in the Materials and Methods section of your manuscript if their identity has been authenticated, state the authentication method (such as STR profiling), and report the mycoplasma contamination testing status. Please consult our Journal Policies for further details: https://submit.elifesciences.org/html/elife author instructions.html\#policies

* Advice about statistical reporting: in your revised paper, please ensure that any statistical analysis methods have been described and justified; ensure that raw data are presented in figures whenever informative to do so (typically when $\mathrm{N}$ per group is less than 10); for each experiment, ensure that you have identified the statistical tests used, the exact values of $\mathrm{N}$, definitions of center, methods of multiple test correction, and dispersion and precision measures; and please report exact $\mathrm{p}$-values wherever possible alongside the summary statistics and $95 \%$ confidence intervals (for all key questions and not only when the $p$-value is less than 0.05 ).

* If your revised article is accepted for publication, please note that authors can choose to have their accepted PDF published by eLife within a few days of acceptance, in advance of the version of record that will be subject to technical processing, editing, and author proofing. Please consider whether this is suitable for your work, so you can select the appropriate option at the time of resubmission. In the event that the study has potentially broad public relevance, we recommend you consult your press officer before making this decision.

* Please also be aware that we will publish the most substantive parts of the decision letter and accompanying author responses at the end of the HTML article, should your article be accepted for publication. In this event you acknowledge and agree that these will be published under the terms of the Creative Commons Attribution license.

Please use the following link when you are ready to resubmit:

[https://submit.elifesciences.org/cgibin/main.plex?el=A4Hz4dhE5A6BSHG3I2A9ftda2P6LITVd7CMIIMHtSdYBQZ]

Best wishes,

Hugo Merchant

Reviewing Editor, eLife

Barbara Shinn-Cunningham

Senior Editor, eLife 


\section{Spanish summary}




\section{Introducción}

La información de nuestro entorno proviene principalmente de nuestros sistemas sensoriales. Una de las principales características de estos sistemas es la detección de estímulos nuevos, elemento común en nuestro sistema nervioso. La capacidad de responder a estímulos nuevos que ocurren en el entorno es de suma importancia para la supervivencia (Malmierca, 2014). La presencia de un estímulo novedoso desencadena una cascada de eventos neuronales que incluyen percepción, atención, aprendizaje y memoria. El sistema auditivo tiene la capacidad de detectar sonidos que rompen la regularidad que establece un flujo de sonido en la escena auditiva (por ejemplo, el sonido de una alarma de emergencia en un restaurante) (Bendixen, 2014; Szabó et al., 2016). Esta capacidad es muy importante para la percepción de nuestro entorno y se ve alterada en algunas enfermedades neurodegenerativas y trastornos neuropsiquiátricos, como por ejemplo en la enfermedad de Alzheimer, la esquizofrenia o el autismo (Hardy et al., 2020; Matthews et al., 2013; Ruzzoli et al., 2016).

En esta tesis, describiré las respuestas generales a la adaptación específica a estímulos en la corteza auditiva de rata. A continuación, describiré cómo la acetilcolina modula esta respuesta frente a estímulos novedosos, cuáles son los receptores colinérgicos asociados a esta modulación y además mostraré cómo la acetilcolina modula los niveles de predicción de error. Finalmente, describiremos cómo las ratas pueden discriminar sonidos novedosos relevantes para el comportamiento. 


\section{Corteza auditiva}

Desde un punto de vista anatómico, podemos definir la corteza auditiva (AC) como cualquier estructura de la corteza cerebral que recibe información del cuerpo geniculado medial del tálamo (MGB). Según esta definición, la AC de la mayoría de los mamíferos estaría ubicada en áreas adyacentes al lóbulo temporal (Malmierca y Hackett; 2010; Hackett, 2015). Podemos clasificar la AC en primaria o secundaria según las proyecciones anatómicas que recibe o por su respuesta electrofisiológica. La corteza auditiva primaria (o lemniscal), presenta una respuesta sintonizada, con gran especificidad a las frecuencias del sonido y latencias cortas. Por el contrario, las neuronas de la corteza auditiva secundaria (o no lemniscal) muestran una respuesta menos específica a las frecuencias del sonido y con latencias de respuesta más largas (Hackett, 2015). Además, existen cortezas asociativas que integran la información auditiva con la de otros sistemas sensoriales (Ghazanfar y Schroeder, 2006).

A diferencia de los centros auditivos subcorticales, donde su estructura y fisiología son relativamente homólogas y están bien conservadas, la estructura y las propiedades fisiológicas de los campos de la corteza auditiva varían sustancialmente entre especies (Hackett, 2015). En la corteza auditiva de rata se han descrito cinco áreas: la corteza auditiva primaria (A1), el área auditiva posterior (PAF), el área auditiva anterior (AAF), el área auditiva ventral (VAF) y el área auditiva suprarrinal (SRAF); basadas en la orientación espacial de mapas tonotópicos, características de ajuste espectral, características de ajuste de intensidad, umbrales de respuesta, latencias de respuesta y una comparación de proyecciones hacia el tálamo auditivo (Nieto-Diego y Malmierca, 2016; Polley et al., 2007; Profant et al. al., 2013). La referencia principal para definir la 
extensión de estos campos es la progresión de sus gradientes tonotópicos característicos. Los límites entre las distintas áreas corticales auditivas están definidos por inversiones o bifurcaciones de estos gradientes. Esta característica clave hace factible localizar la posición relativa de cada campo en un solo animal mediante un mapeo electrofisiológico in vivo (Nieto-Diego y Malmierca, 2016).

Vías lemniscales y no lemniscales en el sistema auditivo

Como se mencionó anteriormente, la información auditiva se transmite a lo largo de varios núcleos organizados jerárquicamente a través del neuroeje auditivo. Sin embargo, desde el mesencéfalo, se han distinguido dos vías principales de características estructurales y funcionales que se diferencian entre sí. Estas vías se han denominado "lemniscal" y "no lemniscal" (Lee y Winer, 2008), que también pueden denominarse regiones primarias 0 no primarias, respectivamente. Las respuestas de la vía lemniscal está impulsada fundamentalmente por las características físicas del sonido. Las divisiones no lemniscales permiten un procesamiento auditivo de orden superior, constituyendo un sistema secundario capaz de procesar aspectos más complejos del análisis de la escena auditiva como el contexto y entorno o la historia del sonido.

En general, la vía lemniscal se origina en el núcleo central del colículo inferior, donde recibe proyecciones de los núcleos del lemnisco lateral, para luego ascender a la división ventral del MGB, proyectando hacia A1, AAF y VAF. La vía no lemniscal, en cambio, recibe proyecciones de múltiples fuentes, incluidas estructuras no auditivas. Surge en las cortezas dorsal, lateral y rostral del colículo 
inferior, proyectando hacia las divisiones dorsal y medial del MGB y finalmente, hacia PAF y SRAF (Carbajal y Malmierca, 2018).

Adaptación específica a estímulos en el sistema auditivo

En el sistema nervioso central existen fundamentalmente dos tipos de respuestas neurales adaptativas cuando presentamos estímulos sensoriales de forma repetitiva: una corresponde al fenómeno de habituación neuronal, donde hay una disminución en la tasa de descarga neuronal generalizada, y que no se recupera inmediatamente cuando presentamos un tren de estímulos (PérezGonzález y Malmierca, 2014). El otro corresponde a la adaptación específica a estímulos (SSA), que ocurre cuando las neuronas disminuyen la respuesta a los estímulos presentados frecuentemente (estándar) pero continúan respondiendo a los estímulos que novedosos (discrepantes).

La SSA ha sido registrada en el colículo inferior, el tálamo y la corteza auditiva (Carbajal y Malmierca, 2018; Carbajal y Malmierca, 2020). Ulanovsky y colaboradores describieron por primera vez la SSA en la corteza auditiva primaria de la rata, utilizando un paradigma tipo oddball (Ulanovsky et al., 2003). Su estudio demostró la presencia de SSA en la corteza auditiva y propuso que era una propiedad exclusivamente cortical; sin embargo, posteriormente se demostró que la SSA también estaba presente tanto en el colículo inferior (PérezGonzález et al., 2005; Malmierca et al., 2009) como en el tálamo auditivo (Antunes et al. 2010). En la corteza auditiva, la SSA se distribuye ampliamente y es ubicua tanto en áreas lemniscales como no lemniscales. Se pueden observar grandes diferencias entre la respuesta neuronal proveniente de ambas áreas. 
Nieto-Diego y Malmierca demostraron que los niveles de SSA son más altos en las zonas no lemniscales de la corteza auditiva de la rata, en comparación a los lemniscales, creando un gradiente topográfico a lo largo de ésta (Nieto-Diego and Malmierca., 2016)

\section{Codificación predictiva}

En los últimos años, la codificación predictiva ha generado mucho interés en la comunidad neurocientífica. Según la teoría de la codificación predictiva, la percepción surge de la integración de información sensorial del entorno y nuestras predicciones estarían basadas en una representación interna de esta información (Friston, 2010, 2005). De esta forma, las áreas corticales de nivel superior generan predicciones sobre el entorno que se envían de arriba hacia abajo, a niveles jerárquicos inferiores, para suprimir la actividad neuronal ascendente evocada por eventos sensoriales que pueden anticiparse. Sin embargo, cuando las predicciones actuales no coinciden con las entradas sensoriales reales, los niveles inferiores generarían errores de predicción ascendentes, de abajo hacia arriba, a los niveles jerárquicos superiores (Friston, 2008; Friston y Kiebel, 2009). Las entradas actuales se predicen a partir de eventos pasados a través de un modelo, y la finalidad del sistema es minimizar los errores en la predicción actualizando continuamente dicho modelo. La reducción del error de predicción se logra mediante interacciones recurrentes entre niveles de una jerarquía de procesamiento, organizados en distintas estructuras anatómicas y poblaciones neuronales (Bastos et al., 2012a). Los modelos computacionales internos que genera nuestro cerebro sopesarían los errores de predicción de la entrada sensorial por su precisión, ya que los errores 
de predicción pueden existir en diferentes niveles de incertidumbre (Parr y Friston, 2017).

En términos neurobiológicos, la precisión está mediada por la acción de los sistemas neuromoduladores, incluido el dopaminérgico (Valdés-Baizabal et al., 2020a) o el colinérgico (Ayala y Malmierca, 2015; Moran et al., 2013). La teoría de la codificación predictiva explicaría el fenómeno de atenuación de la respuesta neuronal cuando un estímulo es repetitivo (supresión de la repetición) o un fuerte aumento de la respuesta cuando la entrada sensorial es distinta a la esperada (error de predicción) (Auksztulewicz y Friston, 2016) y tendría a la adaptación específica a estímulos como su principal correlato a nivel neuronal.

\section{Acetilcolina}

La acetilcolina (ACh) es un neurotransmisor ampliamente distribuido en el sistema nervioso central y fue el primero en ser descrito por Henry Dale y Otto Loewy, por lo que fueron galardonados con el Premio Nobel de Fisiología en 1936 (Loewi, 1921). La acetilcolina se sintetiza a partir de la colina sérica y se compone de dos componentes, acetato y colina, que están unidos por la acción de la acetilcolina transferasa. Existen dos tipos principales de receptores colinérgicos: nicotínicos y muscarínicos. Los receptores nicotínicos permiten la apertura de canales iónicos (receptor de tipo ionotrópico) mientras que los receptores muscarínicos, están mediados por la interacción con proteínas de tipo G (receptor de tipo metabotrópico), que es un poco más lento en su respuesta (Jones et al., 2012). A nivel encefálico, existen dos fuentes principales de acetilcolina. Una se origina en el mesencéfalo (área pedúnculo-pontina y área 
tegmentaria latero-dorsal) las cuales proyectan hacia las regiones subcorticales del cerebro. Por otra parte, la principal fuente de acetilcolina en la corteza auditiva es el prosencéfalo basal (Mesulam, 2013; Villano et al., 2017)

La acetilcolina modula distintos procesos neurobiológicos como la atención, aprendizaje, memoria, sueño y refuerzo cognitivo (Bentley et al., 2011; Hasselmo y Giocomo, 2006; Newman et al., 2012) además de cumplir un rol fundamental en el aprendizaje sensorial (Hasselmo, 1999; Hasselmo y Sarter, 2011; Metherate, 2011; Picciotto et al., 2012; Sarter et al., 2001; Weinberger, 2004). La acetilcolina liberada en el prosencéfalo basal de los mamíferos promueve la activación de los receptores muscarínicos y nicotínicos en las cortezas auditiva, visual y somatosensorial (Disney et al., 2007; Eggermann et al., 2014; Metherate, 2011). En la corteza auditiva, la activación colinérgica de las neuronas VIP+ (vasoactive intestinal peptide, péptido intestinal vasoactivo) puede aumentar la modulación de la ganancia del procesamiento sensorial a través de circuitos desinhibidores (Fu et al., 2014). La acetilcolina optimizaría las respuestas aferentes cuando se recibe información sensorial, disminuyendo el procesamiento intra-cortical (Metherate et al., 1992).

Acetilcolina y adaptación específica a estímulos

Estudios previos realizados en el colículo inferior han demostrado que la acetilcolina aumenta específicamente y de manera diferencial la respuesta al tono estándar (pero no al discrepante) en un paradigma oddball. Este efecto da como resultado una disminución significativa de la SSA y un aumento de los niveles de supresión por repetición. El uso de antagonistas colinérgicos 
(escopolamina y mecamilamina) restituye estos efectos, pero solo la escopolamina lo hace de forma significativa (Ayala y Malmierca, 2015). Además, trabajos previos sugieren que la acetilcolina puede codificar cambios en la precisión de los errores de predicción en las jerarquías corticales sensoriales (Friston, 2008) y modular la precisión de optimización mediante la modulación de ganancia en células piramidales supragranulares en la corteza sensorial primaria (Moran et al., 2013). 


\section{HIPÓTESIS}

Considerando que 1) el sistema nervioso central, y particularmente el sistema auditivo, está organizado jerárquicamente, 2) que, de acuerdo con la teoría de codificación predictiva, se produce una predicción de error creciente desde el colículo inferior hacia la corteza auditiva y 3) que la corteza auditiva recibe una fuerte inervación colinérgica, proponemos la siguiente hipótesis:

La acetilcolina modula los niveles de adaptación específica a estímulos y de predicción de error en la corteza auditiva. Además, también proponemos que la acetilcolina juega un papel clave en la detección de sonidos relevantes en tareas de comportamiento. 


\section{OBJETIVOS}

Para probar esta hipótesis general, presento los siguientes objetivos específicos:

1) Determinar si la adaptación específica a estímulos varía según las distintas áreas de la corteza auditiva

2) Determinar cómo la acetilcolina afecta los niveles de adaptación específica a estímulos a lo largo de las diferentes capas de la corteza auditiva.

3) Determinar qué tipo de receptores colinérgicos están involucrados en la modulación de adaptación-específica a estímulos.

4) Determinar si la acetilcolina modula los errores de predicción y los niveles de supresión de repetición en la corteza auditiva y si juega un papel clave en la precisión de predicción de error

5) Determinar si el entrenamiento auditivo juega un papel en la detección de la saliencia de sonidos relevantes para el comportamiento. 


\section{RESUMEN DE RESULTADOS}

Estudio I: Detección de la novedad en neuronas identificadas fisiológicamente en la corteza auditiva de rata

Objetivo: Determinar si las neuronas excitatorias e inhibitorias de la corteza auditiva en rata anestesiada muestran o no diferencias en la detección de novedad.

Metodología: Se registraron 282 neuronas en la corteza auditiva de ratas anestesiadas (originalmente recolectadas para otros estudios, incluyendo algunos ya publicados: Parras et al., 2019, 2017 ) y se clasificaron como excitatorias o inhibitorias según la forma de los potenciales de accion y su tasa de descarga (de estas 282 neuronas, se utilizaron finalmente 231 para el estudio). Una vez separadas, se analizo su localizacion cortical y profundidad. Finalmente se analizaron los valores de deteccion de novedad, error de predicción y supresión por repetición obtenidos.

Resultados: Un total de 170 neuronas fueron clasificadas como inhibitorias y 61 como excitatorias, distribuidas entre las capas II y VI de la corteza auditiva. De estas 231 neuronas, 205 neuronas fueron localizadas topográficamente (111 neuronas lemniscales y 94 no lemniscales). Tanto las neuronas de tipo excitatorio como las inhibidoras mostraron un nivel importante en el grado de SSA y por lo tanto, ambos tipos intervienen activamente en la detección de novedad. Solo en A1 se observó una diferencia significativa en la detección de novedad. Por otro lado, no detectamos diferencias significativas en los valores de predicción de error, supresión por repetición ni en la detección de novedad entre ambos grupos. 
Conclusiones: Tanto las neuronas excitadoras como inhibidoras en la AC de rata muestran niveles similares en la detección de novedad, por lo que es muy probable que la detección de la novedad se genere a través de redes y circuitos neuronales.

\section{Estudio II: Modulación colinérgica en la adaptación específica a estímulos y niveles de predicción de error en corteza auditiva de rata}

Objetivo: Determinar si la modulación colinérgica modifica los niveles de adaptación específica a estímulos y los niveles de error de predicción en neuronas corticales de ratas anestesiadas.

Metodología: Se registró la actividad específica a estímulos en 99 neuronas localizadas en áreas lemniscales y no lemniscales en la corteza auditiva de ratas anestesiadas (31 animales), antes, durante y después de una inyección microiontoforética de acetilcolina. Además, se registraron 23 neuronas más utilizando antagonistas colinérgicos de tipo muscarínico y nicotínico (escopolamina y mecamilamina, respectivamente). Se evaluaron además los niveles de error de predicción, supresión por repetición y detección de novedad Resultados: Los resultados mostraron una diferencia significativa en los valores de SSA en neuronas tras la inyección de acetilcolina. Este aumento en la adaptación específica se produjo por un aumento en la tasa de descarga frente a los tonos discrepantes de manera específica, sin afectar a las respuestas a los sonidos repetitivos. Por otro lado, los resultados también han demostrado que esta acción está mediada por receptores de tipo muscarínico. Finalmente, la acetilcolina incremento los niveles de detección de novedad y predicción del error 
tanto en áreas lemniscales como no lemniscales pero no los niveles de supresión por repetición.

Conclusiones: La acetilcolina juega un papel modulador clave en la generación de predicción de error incrementando la precisión de éstos y de la SSA

Objetivo: Estudiar el efecto de sonidos relevantes para tareas de comportamiento en la saliencia y respuesta de predicción de error en ratas entrenadas.

Metodología: Se entrenaron 16 ratas utilizando diferentes variantes de una tarea de discriminación auditiva para verificar si la exposición repetida a sonidos relevantes y / o irrelevantes muestra efectos a largo plazo en la representación de esos sonidos en las neuronas de A1. Una vez entrenados los animales, se asignaron aleatoriamente a 4 grupos distintos (4 ratas por grupo). En cada grupo, se utilizaron diferentes pares de frecuencias de sonido, pero el tono repetitivo se mantuvo constante por grupo en todas las sesiones y las variantes de la tarea de comportamiento.

Resultados: Los resultados demostraron que las ratas pueden discriminar con éxito la saliencia de sonidos no repetitivos (con baja probabilidad de aparición) frente a sonidos repetitivos (con alta probabilidad de aparición). Por otra parte, las ratas entrenadas mostraron mayor SSA en tres de los cuatro pares de frecuencias para las cuales fueron entrenadas. Finalmente, se observaron diferencias significativas en los niveles de predicción de error (pero no de supresión por repetición) en el grupo de ratas entrenadas. 
Conclusiones: las ratas pueden discriminar con éxito la saliencia de los estímulos discrepantes. Por otra parte, el entrenamiento en la tarea de discriminación auditiva tuvo efectos sobre la actividad neuronal, aumentando la detección de novedad y la predicción de error (pero no la supresión por repeticion) 


\section{CONCLUSIONES}

1) El análisis morfológico de las espigas neuronales obtenidas a partir de registros extracelulares en la corteza auditiva de rata anestesiada se puede utilizar para clasificar las neuronas como excitadoras o inhibidoras.

2) Tanto las neuronas potencialmente excitadoras como inhibidoras en la corteza auditiva de rata muestran niveles similares de detección de novedad. Estos resultados sugieren la implicación de ambos tipos de neuronas en los circuitos que generan la detección de novedad y que la adaptación específica a estímulos se genera a nivel de redes neuronales.

3) La aplicación local de acetilcolina incrementa la excitabilidad neuronal.

4) La modulación colinérgica en la corteza auditiva aumenta los niveles de adaptación específica a estímulos. Más concretamente, la acetilcolina aumenta los niveles de predicción de error, estableciendo un aumento de ganancia en las unidades de predicción de error para optimizar la precisión predictiva del cerebro.

5) Las ratas entrenadas en una tarea de discriminación auditiva pueden detectar con éxito la saliencia de los sonidos discrepantes de baja probabilidad de aparición, integrados en una secuencia de sonidos repetidos con alta probabilidad de aparición.

6) Las ratas entrenadas en una tarea de discriminación auditiva desarrollan mecanismos de plasticidad neuronal a largo plazo, incrementando la respuesta neuronal a los sonidos relevantes para el comportamiento. 